\title{
Undersampled Radial STEAM MRI Methodological Developments and Applications
}

\author{
Dissertation \\ for the award of the degree \\ "Doctor rerum naturalium" \\ of the Georg-August-Universität Göttingen \\ within the doctoral program \\ Physics of Biological and Complex Systems \\ of the Georg-August University School of Science (GAUSS) \\ submitted by \\ Andreas Merrem \\ born in Darmstadt
}

Göttingen, 2018 


\section{Thesis Committee}

\section{First referee and supervisor}

Prof. Dr. Jens Frahm

Biomedizinische NMR Forschungs GmbH

Max-Planck-Institut für Biophysikalische Chemie, Göttingen

\section{Second referee}

Prof. Dr. Marina Bennati

Forschungsgruppe Elektronenspinresonanz-Spektroskopie

Max-Planck-Institut für Biophysikalische Chemie, Göttingen

Dr. Florian Rehfeldt

3. Physikalisches Institut

Georg-August-Universität Göttingen

\section{Examination Board}

Prof. Dr. Jens Frahm

Prof. Dr. Marina Bennati

Dr. Florian Rehfeldt

PD Dr. Peter Dechent

Medizinische Fakultät

Georg-August-Universität Göttingen

Prof. Dr. Bert de Groot

Forschungsgruppe Computergestützte biomolekulare Dynamik

Max-Planck-Institut für Biophysikalische Chemie, Göttingen

Prof. Dr. Ulrich Parlitz

Forschungsgruppe Biomedizinische Physik

Max-Planck-Institut für Dynamik und Selbstorganisation, Göttingen

Date of examination: 5 March 2018 
Für Eva 


\section{Contents}

\begin{tabular}{llr}
\hline 1 & Introduction & 7 \\
\hline
\end{tabular}

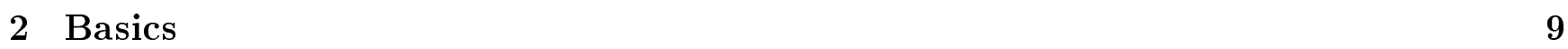

2.1 Magnetic resonance imaging (MRI) . . . . . . . . . . . . . . . . . 9

$2.1 .1 \quad$ Nuclear magnetic resonance . . . . . . . . . . . . . . . . . . 9

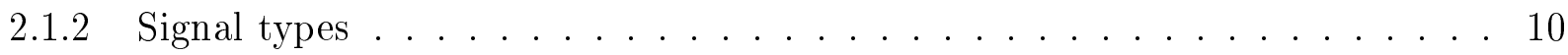

2.1 .3 Spatial encoding . . . . . . . . . . . . . . . . . 14

2.1 .4 Echo-planar imaging . . . . . . . . . . . . . . . . . . 18

$2.1 .5 \quad$ Chemical shift selective (CHESS) imaging . . . . . . . . . . . . 18

2.2 Diffusion-weighted MRI $\ldots \ldots \ldots \ldots \ldots \ldots \ldots \ldots$

2.2 .1 Diffusion in human tissue . . . . . . . . . . . . . . . . . . 19

2.2 .2 The diffusion-weighted spin echo . . . . . . . . . . . . . . . . 20

2.2 .3 MRI measurements of the diffusion tensor . . . . . . . . . . . . . . . . 21

2.3 Image reconstruction by nonlinear inversion . . . . . . . . . . . . . . . . . 22

2.4 Use of resources . . . . . . . . . . . . . . . . . . . . . . . . . 24

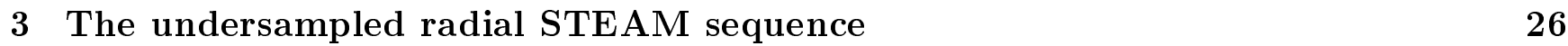

3.1 Sequence design . . . . . . . . . . . . . . . . . . . . 26

3.1 .1 From STEAM to single-shot STEAM . . . . . . . . . . . . . . . . . 26

3.1 .2 The radial single-shot STEAM sequence . . . . . . . . . . . . . . 27

$3.1 .3 \quad$ Variable flip angle scheme . . . . . . . . . . . . . . . . . 28

3.1 .4 Spoiling in the readout train . . . . . . . . . . . . . . . 30

3.1 .5 Discussion . . . . . . . . . . . . . . . . . . 33

3.2 Cardiac black-blood MRI using undersampled radial STEAM. . . . . . . . . . . . . 33

3.2 .1 Background . . . . . . . . . . . . . . . . 33

3.2 .2 Methods . . . . . . . . . . . . . . . . . . . 34

3.2 .3 Results . . . . . . . . . . . . . . . . . . . 35

3.2 .4 Discussion . . . . . . . . . . . . . . . . . 36

4 Diffusion-weighted STEAM MRI of the brain 38

4.1 Introduction . . . . . . . . . . . . . . . . . . . 38

4.2 The diffusion-weighted undersampled radial single-shot STEAM sequence . . . . . 39

4.2 .1 Sequence design . . . . . . . . . . . . . . . . . 39

4.2 .2 Multi-slice imaging . . . . . . . . . . . . . . . . . 39

4.3 Optimization of image acquisition parameters . . . . . . . . . . . . . . . 40

4.3 .1 Fat suppression . . . . . . . . . . . . . . . . . 41

4.3 .2 Spoiling of the spin echo . . . . . . . . . . . . . . . . 42 
$4.3 .3 \quad$ FID spoiling . . . . . . . . . . . . . . . . . . . . . . . . . . 43

4.3 .4 Repetition time $\ldots \ldots \ldots \ldots$. . . . . . . . . . . . . . . . . . 43

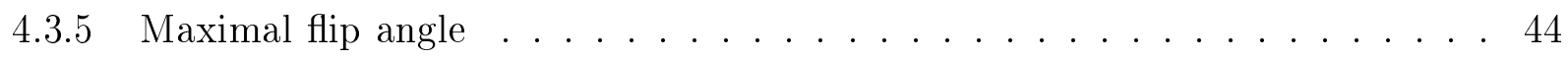

4.4 Image reconstruction $\ldots \ldots \ldots \ldots \ldots \ldots \ldots \ldots$

4.4 .1 Methods . . . . . . . . . . . . . . . . . . . . . . . 46

4.4 .2 Numerical experiments $\ldots \ldots \ldots \ldots \ldots \ldots \ldots \ldots \ldots \ldots$

4.4 .3 Discussion . . . . . . . . . . . . . . . . . . . . . . 56

4.5 In vivo studies using optimized imaging protocols $\ldots \ldots \ldots \ldots \ldots$. . . . . . 56

4.5 .1 Methods . . . . . . . . . . . . . . . . . . . 56

4.5 .2 Results $\ldots \ldots \ldots \ldots \ldots \ldots \ldots$

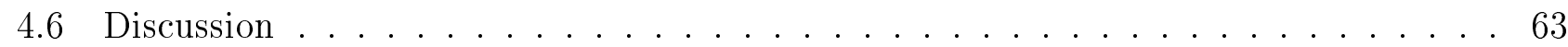

5 Diffusion-weighted STEAM MRI of the prostate $\quad 66$

5.1 Introduction . . . . . . . . . . . . . . . . . . 66

5.2 Methods . . . . . . . . . . . . . . . . . . . . . . 67

5.2 .1 ADC measurements from data with low SNR $\ldots \ldots \ldots$. . . . . . 67

5.2 .2 Multi-shot image reconstruction $\ldots \ldots \ldots \ldots$. . . . . . . . 67

5.2 .3 Validation of the reconstruction algorithm $\ldots \ldots \ldots \ldots \ldots$

5.2 .4 Optimization of imaging parameters . . . . . . . . . . . . . 70

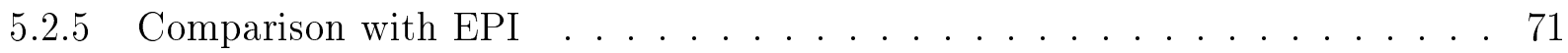

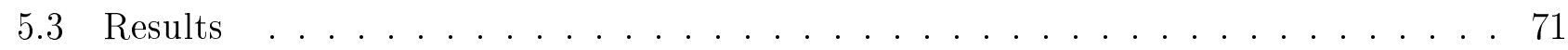

5.3 .1 Image reconstruction: numerical phantom $\ldots \ldots \ldots . \ldots 71$

5.3 .2 Image reconstruction: In vivo imaging of the prostate $\ldots . . . . . .74$

$5.3 .3 \quad$ Parameter optimization for multi-shot prostate imaging . . . . . . . . . . 79

5.3 .4 Comparison with EPI $\ldots \ldots \ldots \ldots \ldots \ldots \ldots \ldots \ldots$

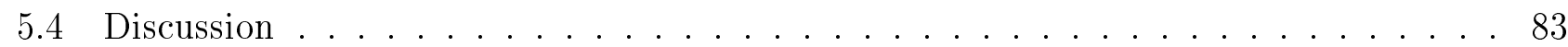

6 Diffusion-weighted STEAM MRI using inner-volume excitation 86

6.1 Introduction . . . . . . . . . . . . . . . . . 86

6.2 Methods . . . . . . . . . . . . . . . . . . . . . . . 86

$6.2 .1 \quad$ Imaging sequence $\ldots \ldots \ldots \ldots \ldots$. . . . . . . . . . . . 86

6.2 .2 Characterization of signal losses $\ldots \ldots \ldots \ldots$. . . . . . . . . . 89

6.2 .3 In-vivo experiments $\ldots \ldots \ldots \ldots \ldots \ldots \ldots \ldots$

6.3 Results . . . . . . . . . . . . . . . . . . . . . . . . 93

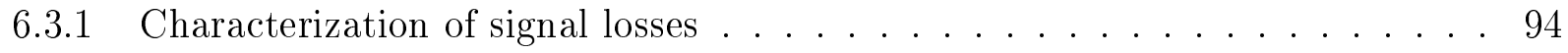

6.3 .2 In-vivo experiments . . . . . . . . . . . . . . . . . . . 96

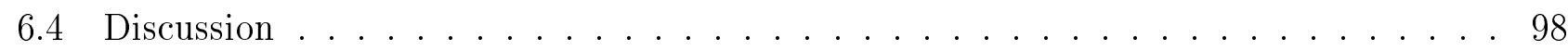


\begin{tabular}{llr}
7 & Summary and outlook & 100 \\
\hline
\end{tabular}

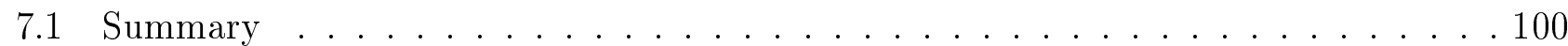

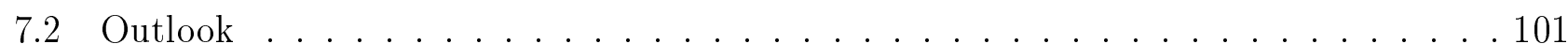

\begin{tabular}{ll}
\hline Abbreviations & 102
\end{tabular}

\begin{tabular}{lr}
\hline References & 104
\end{tabular}

\begin{tabular}{lr}
\hline Curriculum Vitae & 110
\end{tabular}

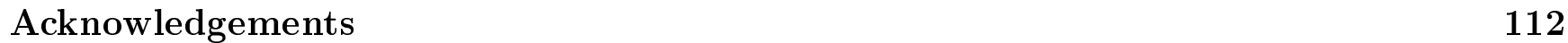




\section{Introduction}

Since the first imaging experiments using nuclear magnetic resonance [40], magnetic resonance imaging (MRI) has developed into a technology which is widely used in the life sciences and for medical diagnostic purposes. It allows for the noninvasive acquisition of images of arbitrary anatomical locations in the human body and with better soft-tissue contrast than available by $\mathrm{X}$-ray modalities. Moreover, a large variety of acquisition and reconstruction techniques provide many different types of information on tissue structure and physiological functions.

As a clinically highly relevant technique diffusion-weighted (DW) MRI [41, 44] offers image contrast which is determined by the degree to which water molecules can diffuse freely in biological tissue on a scale of micrometers. Such measurements offer information about the microscopic structure and dynamics of normal and pathologically altered tissues and therefore lead to a broad range of clinical and scientific applications. In particular, DW MRI of the brain serves important diagnostic problems dealing with ischemic (stroke) and structural lesions (tumors). Furthermore, extended DW-MRI techniques may be used for virtual nerve fiber tractography, which exploits the directional information of water diffusion to identify the main orientations of nerve fibers within an image voxel. More recently, improvements of both MRI hardware and software have expanded the application of DW MRI to locations outside the brain, although such studies remain challenging because of their high sensitivity to motion and an inherently low signal-to-noise ratio (SNR). Respective clinical developments mainly target oncological applications, because the diffusion contrast reflects tissue cellularity and therefore relates to more specific pathological changes of cellular structures than conventional MRI contrasts based on proton density and tissue-dependent NMR relaxation times.

So far, single-shot echo-planar imaging (EPI) [43] is the only technique which is used for routine clinical DW MRI due to its fast acquisition mode and a relatively high SNR. On the other hand, a major drawback of EPI is the inherent sensitivity to magnetic field inhomogeneities which in occur due to local differences in tissue susceptibilities. These problems cause image artifacts such as geometric distortions and altered image intensities which may lead to diagnostic uncertainties or even errors in affected anatomical regions [36].

An alternative technique for DW MRI is the DW single-shot STEAM sequence [47], which allows for image acquisitions without any susceptibility-related artifacts. A proof-of-principle study showed that this approach may avoid diagnostic problems in DW MRI of stroke patients [36]. However, a routine clinical use is precluded because of its lower SNR in comparison to EPI-based techniques.

A distinct property of single-shot STEAM MRI sequences is that reducing the number of spatially encoded data lines allows for a higher (rather than lower) SNR. This special feature strongly motivates the combination of single-shot STEAM sequences with recent developments in undersampled image reconstruction. A particularly efficient method for data undersampling is the 
use of radial sampling trajectories, i.e. the sampling of lines in Fourier space which cross the origin at different angles. This acquisition approach may be advantageously combined with nonlinear inverse image reconstruction (NLINV) as developed in our group by Uecker et al. [62, 63] for real-time MRI.

The central goal of this thesis is the development of a novel DW single-shot STEAM MRI technique which overcomes previous deficiencies. In particular, this should be achieved by exploiting the concepts underlying undersampled radial MRI acquisitions and respective iterative image reconstruction methods. A specific purpose is to increase the SNR and overall image quality of DW single-shot STEAM MRI studies and thereby solve a serious clinical problem with DW-MRI techniques based on EPI. The thesis covers the following topics:

A first step was devoted to the development of an optimized single-shot STEAM technique with a modified sequence design compared to a previous proposal [6] and to implement the method on a human 3 Tesla MRI system. The combination of this non-DW single-shot STEAM sequence with NLINV reconstruction was applied to cardiac black-blood imaging.

Secondly, the technical development was extended to a DW version of the single-shot STEAM MRI sequence and optimized for applications to the human brain. This process involved a modification of the NLINV reconstruction algorithm [60, 62, and the exploration of a large set of experimental parameters for the measurement of brain tissue. A study of healthy volunteers and a small number of pilot patients demonstrated the robustness and excellent image quality of this new DW-MRI modality and indicated significant potential for a widespread clinical use without susceptibility problems.

The third topic dealt with the development of a DW single-shot STEAM MRI technique for studies of the human prostate which represents the most important clinical application of DW MRI outside the brain. Due to major susceptibility-induced artifacts of respective single-shot EPI measurements at the boundary of the prostate [42], the benefit of the STEAM technique from avoiding such artifacts is expected to be even greater than in the brain. To overcome the specifically low SNR in prostate MRI which is partly caused by the desire to use remote radiofrequency coils, the present development involved a novel multi-shot (rather than single-shot) acquisition scheme and a joint nonlinear inverse reconstruction technique with a new regularization method.

A final project addressed the development of a special variant of the DW single-shot STEAM MRI technique for prostate imaging which allows for studies of an inner volume without image aliasing artifacts. This was achieved by the integration of spatially confined radiofrequency excitation pulses into the STEAM sequence. The method is intended to ameliorate problems with off-resonance effects and tissue motion which are predominant in abdominal MRI.

The following section provides introductions into the basic concepts of MRI, into the special features of DW MRI, and into iterative image reconstruction techniques. 


\section{Basics}

\subsection{Magnetic resonance imaging (MRI)}

Magnetic resonance imaging (MRI) is a medical imaging modality which relies on the acquisition the NMR signal of atoms in human tissue using sequences of electromagnetic pulses. This subsection describes the basic principles of MRI as far as they are relevant for this thesis. More detailed descriptions of every topic in this subsection can be found in relevant textbooks [19, 50].

\subsubsection{Nuclear magnetic resonance}

A spin ensemble in a static magnetic field Atoms with an odd number of either protons or neutrons have a nuclear spin $\vec{I}$. For a proton, the spin quantum number is $I=\frac{1}{2}$. Thus, the magnetic quantum number $m$ which characterizes the projection of the spin vector on a quanti-

zation axis is either $\frac{1}{2}$ or $-\frac{1}{2}$. Due to its spin, a proton possesses a magnetic moment $\vec{\mu}=\gamma \vec{I}$ with the gyromagnetic ratio $\gamma=267,5 \frac{M H z}{T}$. An external magnetic field $\vec{B}_{0}=B_{0} \vec{e}_{z}$ induces a Zeeman splitting of energy levels for the magnetic quantum numbers, with an energy difference of $\Delta E=\hbar \omega_{0}$ with the Larmor frequency $\omega_{0}=\gamma B_{0}$. In thermodynamic equilibrium, the ratio of populations of the two energy levels is given by the Boltzmann distribution. For an ensemble of spins, the linear approximation for $\Delta E \ll k_{B} T$ yields the macroscopic magnetization

$$
\vec{M}=\frac{1}{V} \sum_{i} \vec{\mu}_{i}=\rho \frac{\gamma^{2} \hbar^{2}}{4 k_{B} T} \vec{B}_{0}
$$

with the spin density $\rho$.

Excitation The dynamics of the macroscopic magnetization in the presence of an external magnetic field are described by the Bloch equation [4]

$$
\frac{d \vec{M}}{d t}=\gamma \vec{M} \times \vec{B}+\left(\begin{array}{c}
-\frac{M_{x}}{T 2} \\
-\frac{M_{y}}{T 2} \\
\frac{M_{0}-M_{Z}}{T 1}
\end{array}\right)
$$

On time scales which are small against T1 and T2 and with $\vec{B}=\vec{B}_{0}$, the Bloch equation describes precession around the static magnetic field with the Larmor frequency. Hence, excitation requires an oscillating magnetic field which is resonant with the Larmor frequency. The effect of a radiofrequency pulse

$$
\overrightarrow{B_{1}}=\left(\begin{array}{cl}
B_{1}(t) & \cos (\omega t) \\
B_{1}(t) & \sin (\omega t) \\
0
\end{array}\right)
$$

with the envelope $B_{1}(t)$ in combination with the $\vec{B}_{0}$ field can be understood a reference frame 
rotating around the $\mathrm{z}$ axis with the Larmor frequency. The corresponding transformation for the magnetic field is

$$
\vec{B}_{l a b}=\left(\begin{array}{c}
B_{1}(t) \cos (\omega t) \\
B_{1}(t) \sin (\omega t) \\
B_{0}
\end{array}\right) \longrightarrow \vec{B}_{e f f}=\left(\begin{array}{c}
B_{1}(t) \cos \left(\left(\omega-\omega_{0}\right) t\right) \\
B_{1}(t) \sin \left(\left(\omega-\omega_{0}\right) t\right) \\
0
\end{array}\right)
$$

with the magnetic field represented as $\vec{B}_{l a b}$ in the laboratory frame and $\vec{B}_{\text {eff }}$ in the rotating frame. With $\omega=\omega_{0}$ and the physically correct approximations $\frac{1}{\omega_{0}} \ll T 1$ and $\frac{1}{\omega_{0}} \ll T 2$, equation 1 yields a rotation of the magnetization around the y-axis by the flip angle $\alpha=\gamma \int B_{1}(t) d t$. In MRI, radiofrequency pulses are generally characterized by this flip angle, e.g. a pulse with a $\alpha=90^{\circ}$ is called a $90^{\circ}$ pulse.

Relaxation The second term of the Bloch equation describes the relaxation of the magnetization, i.e. its return to the ground state after excitation. Without any radiofrequency pulse, the relaxing magnetization in the rotating frame can be described as

$$
\vec{M}(t)=\left(\begin{array}{c}
M_{\perp}(t) \cos (\varphi) \\
M_{\perp}(t) \sin (\varphi) \\
M_{\|}(t)
\end{array}\right)
$$

with $M_{\perp}(t)=M_{\perp}(t=0) e^{-\frac{t}{T 2}}$ and $M_{\|}(t)=M_{0}+\left(M_{\|}(t=0)-M_{0}\right) e^{-\frac{t}{T 1}}$. Relaxation of the longitudinal magnetization is caused by energy exchange with the local environment. Therefore, the time constant T1 is called the spin-lattice relaxation time. In human tissue, it is in the order of $1 \mathrm{~s}$. The irreversible relaxation of the transverse magnetization is caused primarily by fluctuations of the Larmor frequency due to interactions between spins. This leads to "dephasing", a broadening of the distribution of the phase $\varphi$ which converges to a uniform distribution where $M_{\perp}=0$ on a macroscopic scale. The time constant T2, which is typically in the order of $100 \mathrm{~ms}$ in human tissue, is called spin-spin relaxation time.

The NMR signal is acquired using coil elements which are placed in the vicinity of the sample. Electromagnetic induction leads to an alternating voltage in these coils, with a frequency distribution equal to the distribution of Larmor frequencies in the sample and an amplitude proportional to the transverse magnetization.

\subsubsection{Signal types}

Free induction decay The simplest form of NMR signal acquisition is a $90^{\circ}$ pulse directly followed by a readout of the signal. The resulting signal is the free induction decay (FID), see figure 1. Its envelope describes the exponential decay of the signal due to dephasing (figure 2 of the transverse magnetization. Apart from the irreversible fluctuations of the Larmor frequency 
which lead to spin-spin relaxation, this decay has a reversible component due to stationary, spatial inhomogeneities of the Larmor frequency. These are caused by a spatially varying magnetic susceptibility in the tissue as well as inhomogeneities of the $B_{0}$ field due to imperfections of the hardware. Therefore, the observed decay of the transverse magnetization decays is $M_{\perp}(t)=M_{\perp}(t=0) e^{-\frac{t}{T 2^{*}}}$ with the effective spin-spin relaxation time $T 2^{*}<T 2$.

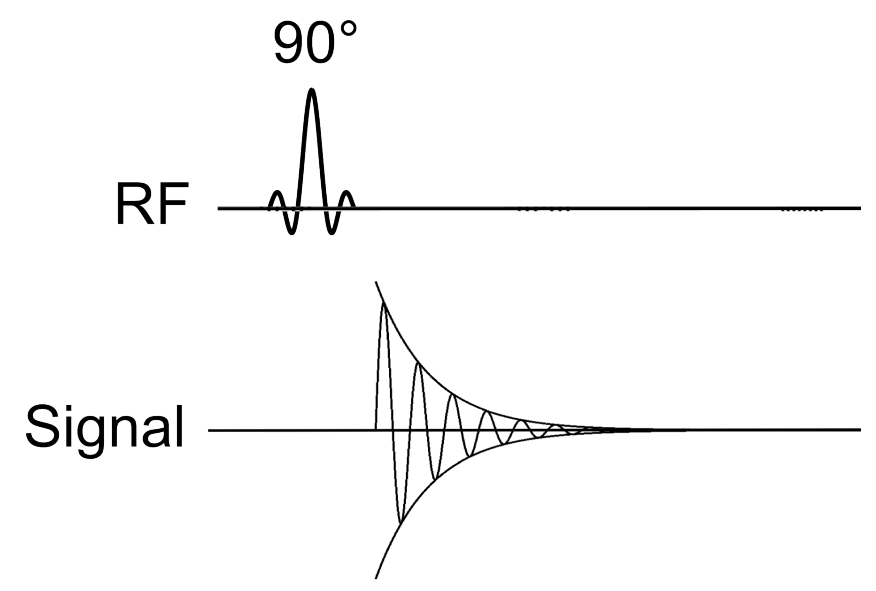

Figure 1 - The free induction decay. After excitation, a damped oscillating signal is observed, with a frequency distribution equal to the distribution of Larmor frequencies. The envelope describes an exponential decay with the time constant $\mathrm{T} 2 *$.

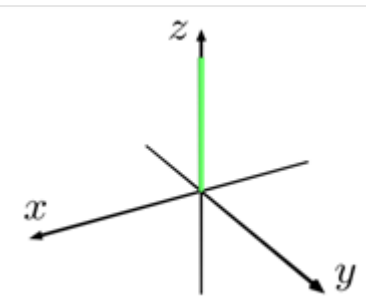

(a) Ground state

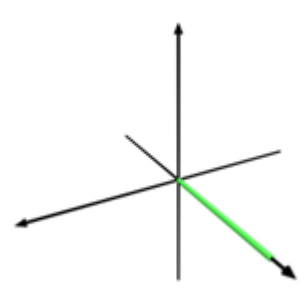

(b) Excitation

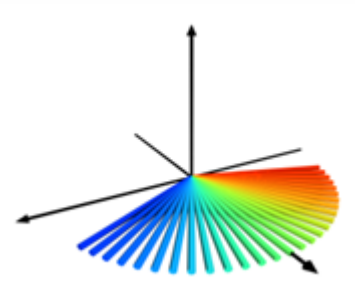

(c) Dephasing

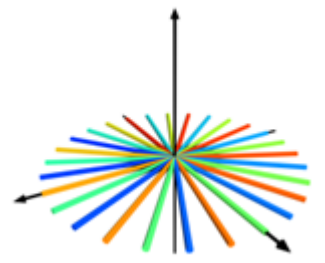

(d) Final state

Figure 2 - Dephasing of the FID signal. After the $90^{\circ}$ pulse which flips the magnetization into the transverse plane, spatial inhomogeneities of the Larmor frequency cause a broadening of the distribution of phases. Visualizations from ref. [46].

Gradient echo In MRI, magnetic field gradients are used to create a dependence of the longitudinal magnetic field on the location. A gradient $\vec{G}$ results in a magnetic field $\vec{B}=\overrightarrow{B_{0}}+\vec{G} \cdot \vec{x} \vec{e}_{z}$, hence the altered Larmor frequency $\omega_{L}=\omega_{0}+\gamma \vec{G} \cdot \vec{x}$. A time-dependent magnetic field gradient 
$\vec{G}(t)$ therefore leads to a spatially and temporally dependent phase of the spins,

$$
\varphi(\vec{x}, t)=\int_{0}^{t}\left(\omega_{L}\left(\vec{x}, t^{\prime}\right)-\omega_{0}\right) d t^{\prime}=\int_{0}^{t} \gamma \vec{G}(t) \cdot \vec{x} d t^{\prime}
$$

If a gradient is switched on after a $90^{\circ}$ pulse as shown in figure 3 , the dephasing effect which follows from equation 2 leads to a far more rapid signal decay than the T2* decay of the FID signal. A subsequent gradient switched in the opposite direction reverts this dephasing effect, hence the signal strength increases again. At the echo time $T E$ after the excitation pulse, the total time integral of the gradient is 0 , hence the gradient-induced phase $\varphi=0$ (equation 2). Therefore, the signal strength at the echo time is only influenced by those dephasing effects which cause the decay of the FID signal, $S(t=T E) \sim e^{-\frac{T E}{T 2 *}}$.

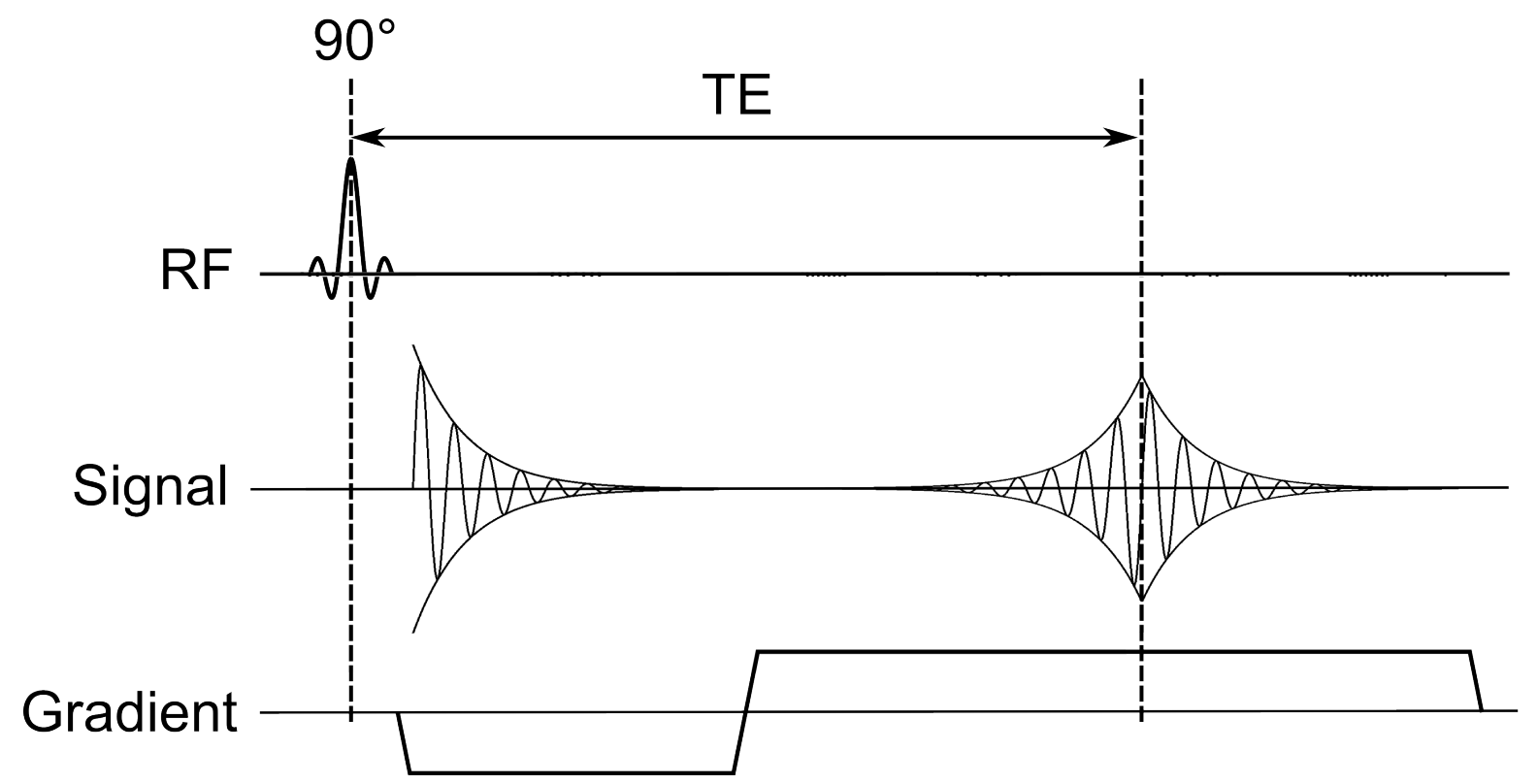

Figure 3 - The gradient echo. A magnetic field gradient leads to very fast and reversible dephasing of the transverse magnetization. The opposite gradient reverts this dephasing effect and creates an echo.

Spin echo A spin echo [22] is generated by a $90^{\circ}$ pulse is followed by a $180^{\circ}$ pulse (see figure 4 ). After the $90^{\circ}$ pulse, the transverse magnetization decays with a time constant $\mathrm{T} 2^{*}$ as for the FID. Those phase differences which are caused by stationary inhomogeneities of the Larmor frequency are inverted with the $180^{\circ}$ pulse after a time period of $T E / 2$ and thus vanish after the echo time $T E$. This effect is visualized in figure 5. The amplitude of the signal at the echo time is only affected by the irreversible dephasing, $S(t=T E) \sim e^{-\frac{T E}{T 2}}$. 


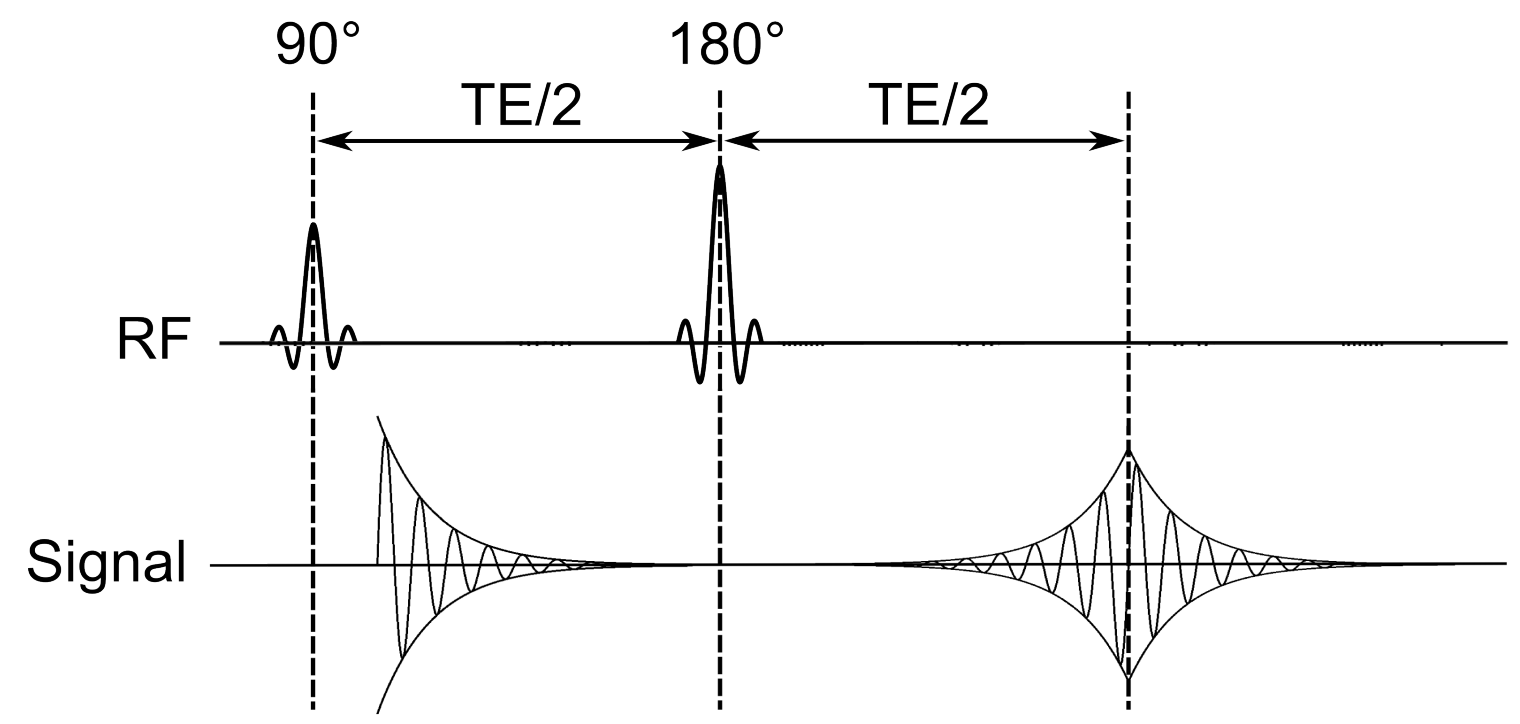

Figure 4 - The spin echo. A $90^{\circ}$ pulse is followed by a $180^{\circ}$. This leads to a partial rephasing of spins, causing a subsequent increase in the MR signal amplitude, which has a peak at the echo time TE.

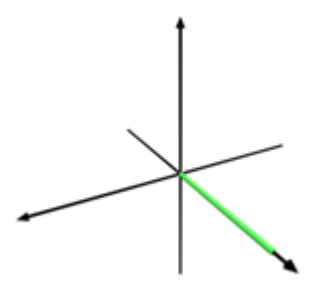

(a) Excitation

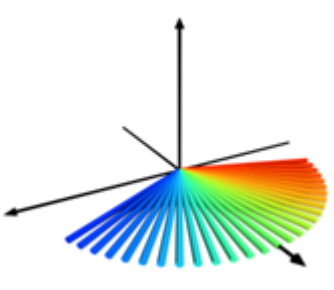

(b) Dephasing

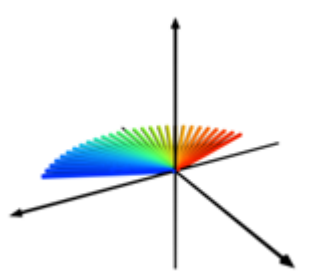

(c) Rephasing

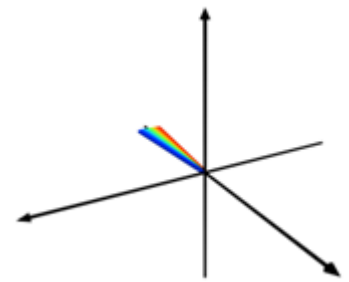

(d) Spin echo

Figure 5 - Formation of a spin echo. After the $90^{\circ}$, local inhomogeneities of the Larmor frequency dephase the magnetization. The $180^{\circ}$ pulse inverts the relative phases caused by the different Larmor frequencies. This leads to rephasing. Visualizations from [46].

Stimulated echo A stimulated echo [22], in its basic form, is created by three $90^{\circ}$ pulses (see figure 6), although other choices of flip angles will also produce stimulated echoes with reduced amplitudes. Like the spin echo, it is an echo which is refocused by radiofrequency pulses and not by gradient pulses. The reversible phase differences which arise after excitation with the first $90^{\circ}$ pulse are refocused by a combination of the second and third $90^{\circ}$ pulse instead of one $180^{\circ}$ pulse. However, the distribution of isochromats, i.e. components of the magnetization with identical Larmor frequencies, evolves in a more complex way, which is visualized in figure 7 . After the excited magnetization from the first $90^{\circ}$ pulse is fully dephased, and after a time $T E / 2$, a second $90^{\circ}$ pulse tilts the isochromats into a longitudinal plane. After transverse dephasing of this state, the phases which the isochromats had acquired prior to the second $90^{\circ}$ pulse remain stored in 
the longitudinal direction (figure 7f]. This state has been called "prepared magnetization" [16]. The third pulse, applied after the "mixing time" TM following the second pulse, tilts the prepared distribution of isochromats by $90^{\circ}$. Hence the stored phase distribution is released into a transverse orientation again, and rephasing of the isochromats leads to an echo after $T E / 2$. Unlike the spin echo, the stimulated echo does not lead to a rephasing of all isochromats, because dephasing between the second and third pulse leads to a distribution of isochromat orientations over the entire unit sphere, which cannot be fully refocused after the third pulse. Without relaxation effects, the amplitude of the stimulated echo is half of that of the spin echo [16]. The dependence of the stimulated echo amplitude on the relaxation times is given by $S(t=T E+T M) \sim e^{-\frac{T E}{T 2}-\frac{T M}{T 1}}$.

A necessary condition for the formation of a stimulated echo is a complete dephasing of the magnetization after each of the first two RF pulses and of the FID signal of the third pulse. This is accomplished by gradients, whose time integrals must be equal in the two $T E / 2$ intervals for rephasing of the stimulated echo.

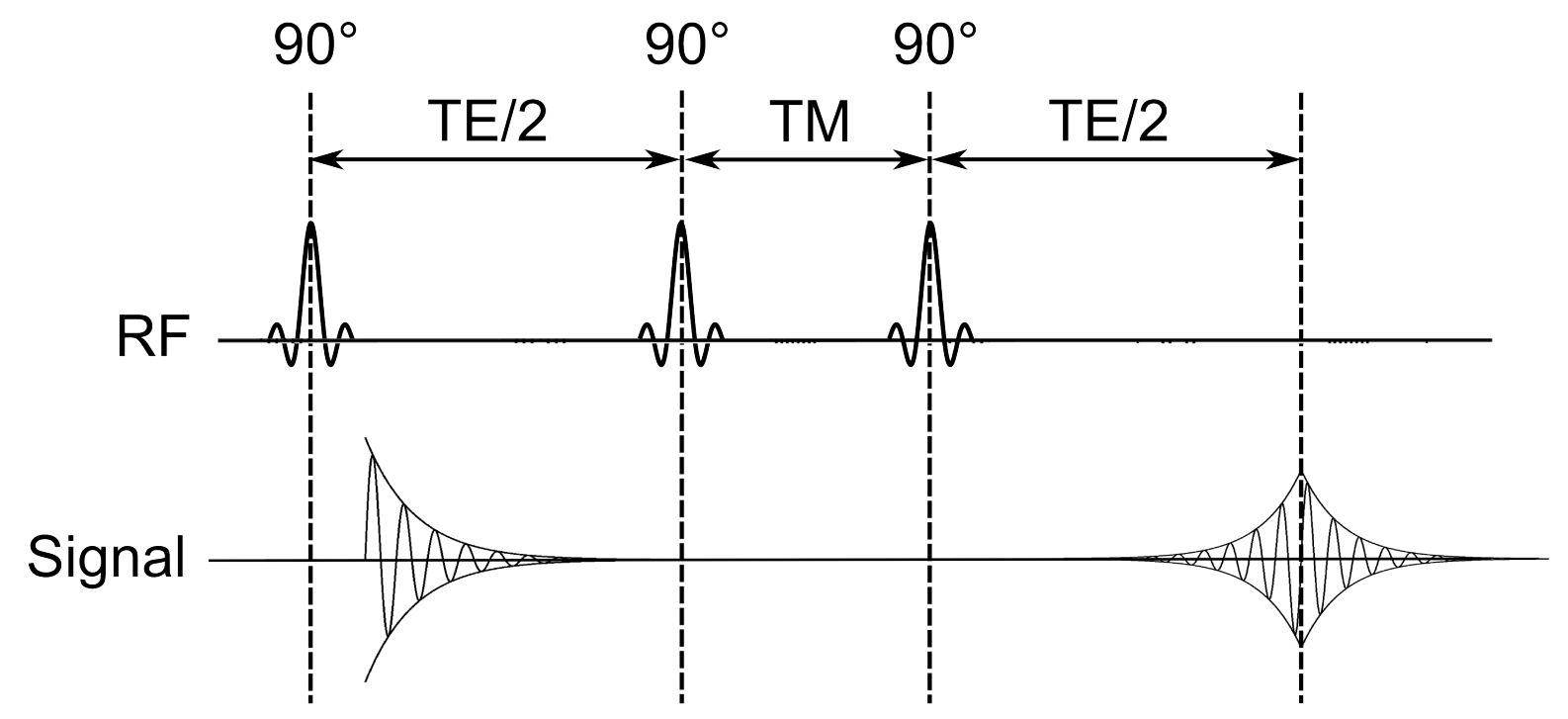

Figure 6 - The stimulated echo. The reversible dephasing which takes place between the first two $90^{\circ}$ pulses is reverted after the third $90^{\circ}$ pulse. This leads to an echo.

\subsubsection{Spatial encoding}

Magnetic field gradients allow the spatially resolved measurement of the transverse magnetization which is determined by the design of the pulse sequence. Spatial encoding is based on two mechanisms: slice-selection and Fourier encoding. To describe these mechanisms, magnetic fields of RF pulses and magnetizations in the transverse plane are represented as complex-valued quantities, with the real part representing the projection on the $\mathrm{x}$-axis and the imaginary part representing the projection on the $\mathrm{y}$-axis, both perpendicular to the static magnetic field. 


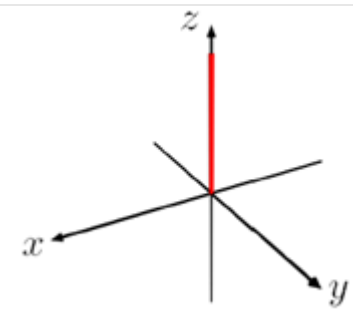

(a) Ground state

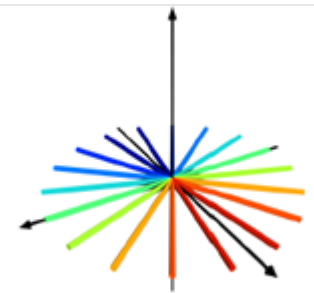

(d) Before second $90^{\circ}$ pulse

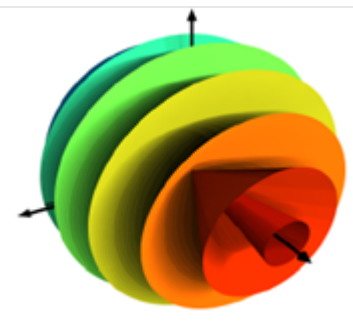

(g) Third $90^{\circ}$ pulse

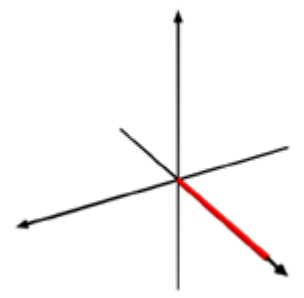

(b) First $90^{\circ}$ pulse

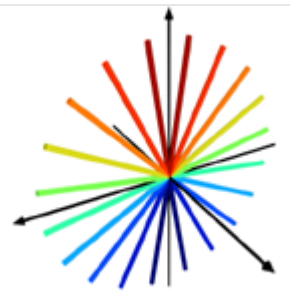

(e) Second $90^{\circ}$ pulse

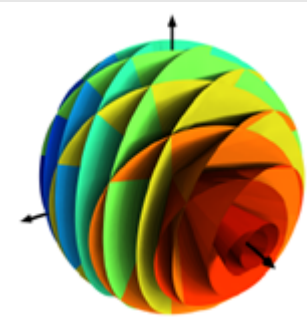

(h) Rephasing

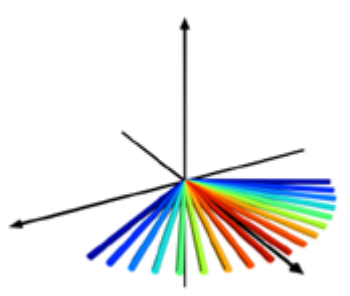

(c) Dephasing

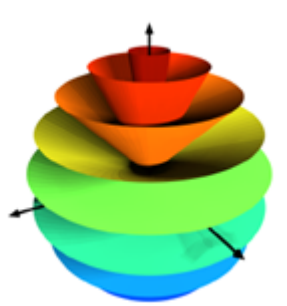

(f) Dephasing (prepared magnetization)

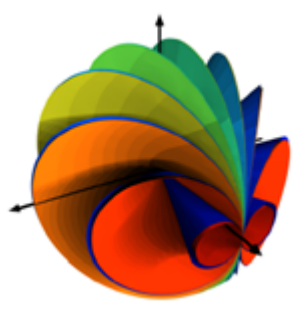

(i) Stimulated echo

Figure 7 - Formation of a stimulated echo. The first $90^{\circ}$ pulse flips the magnetization into the transverse plane. After complete dephasing, the second $90^{\circ}$ pulse brings the isochromats into a longitudinal plane. From there, the magnetization dephases into a pattern which can be visualized by isochromatic cones (sub-figure f). After a third $90^{\circ}$ pulse, partial rephasing takes place. Visualizations from [46].

Slice selection For the selective excitation of a slice of tissue, a radiofrequency pulse of the form $B_{R F}(t)=B_{1}(t) e^{i \omega_{e x} t}$ is superimposed with a gradient $\vec{G}$ in the direction normal to the slice (w.l.o.g. the z-direction). The Larmor frequency is given by $\omega_{L}(z)=\omega_{0}+\gamma G z$. Therefore, frequency distribution of the RF pulse, $\mathcal{F}\left[B_{R F}(t)\right](\omega)=\mathcal{F}\left[B_{1}(t)\right](\omega) \circledast \delta\left(\omega-\omega_{e x}\right)$ determines the slice profile, a relative measure of $\left|M_{\perp}(z)\right|$ after the RF pulse. The slice position is given by $z=\frac{\omega_{e x}-\omega_{0}}{\gamma G}$, and the slice thickness is given by $\Delta z=\frac{\Delta \omega}{\gamma G}$, with $\Delta \omega$ the frequency bandwidth of the envelope $B_{1}(t)$ of the RF pulse. A practical trade-off between an approximation of the desired rectangular slice profile and reasonable pulse durations can be achieved by choosing a Hamming-windowed sinc-function for the envelope of the RF pulse. For a fixed gradient strength, this trade-off can be modified by changing bandwidth-time product (BWTP) of $B_{1}(t)$, which determines the number of side lobes of the sinc-function to be included in the pulse. 
Fourier encoding After the selective excitation of the spins in one slice of tissue, 2-dimensional spatial encoding is necessary to create an MR image of this slice. This is achieved with magnetic field gradients in the in-plane directions. The signal amplitude measured in the receive coils after excitation is proportional to:

$$
S(t)=\int M_{\perp}(\vec{x}, t) d V=\int\left|M_{\perp}(\vec{x}, t)\right| e^{i \varphi(\vec{x}, t)} d V .
$$

Equation 2 for the gradient-induced phase $\varphi$ yields

$$
S(t)=\int\left|M_{\perp}(\vec{x}, t)\right| e^{i\left(\varphi_{0}(\vec{x}, t)+\gamma \int_{o}^{t} \vec{G}\left(t^{\prime}\right) d t^{\prime} \cdot \vec{x}\right)} d V .
$$

This proves that signal acquisition samples the 2-dimensional Fourier transform of the distribution $\left|M_{\perp}(\vec{x}, t)\right| e^{i \varphi_{0}(\vec{x}, t)} \equiv M_{1}(\vec{x}, t)$ w.r.t. the spatial coordinates,

$$
S(t)=\int M_{1}(\vec{x}, t) e^{i \vec{k}(t) \cdot \vec{x}} d V
$$

with the spatial frequency sampled by signal acquisition at time point $\mathrm{t}$ given by

$$
\vec{k}(t)=\gamma \int_{o}^{t} \vec{G}\left(t^{\prime}\right) d t^{\prime}
$$

Thus, the acquisition of the NMR signal in the presence of magnetic field gradients samples the MR image in Fourier space, or "k-space". For discrete sampling, it follows from the properties of the Fourier transform that the field of view $(F O V)$, i.e. the width of the acquired image, is determined by the distance $\Delta k$ between sampled k-space points, $F O V=\frac{1}{\Delta k}$, and the resolution $\Delta x$ is determined by the the maximal spatial frequency $k_{\max }$ which is sampled, $\Delta x=\frac{1}{2 k_{\max }}$. An additional important imaging parameter is the bandwidth per pixel (BW), i.e. the difference between the temporal frequencies in the acquired signal which are mapped to adjacent pixels. Low receiver bandwidths lead to a high signal-to-noise ratio. $S N R \sim \frac{1}{\sqrt{B W}}[19]$. However, a high bandwidth per pixel makes the image acquisition technique more robust to any unwanted variations of the Larmor frequency, and also allows faster image acquisition, because the minimal temporal frequency to be sampled determines the duration of the readout.

K-space can, in principle, be sampled with any trajectory. The most common sampling trajectories are shown in figure 8 . 


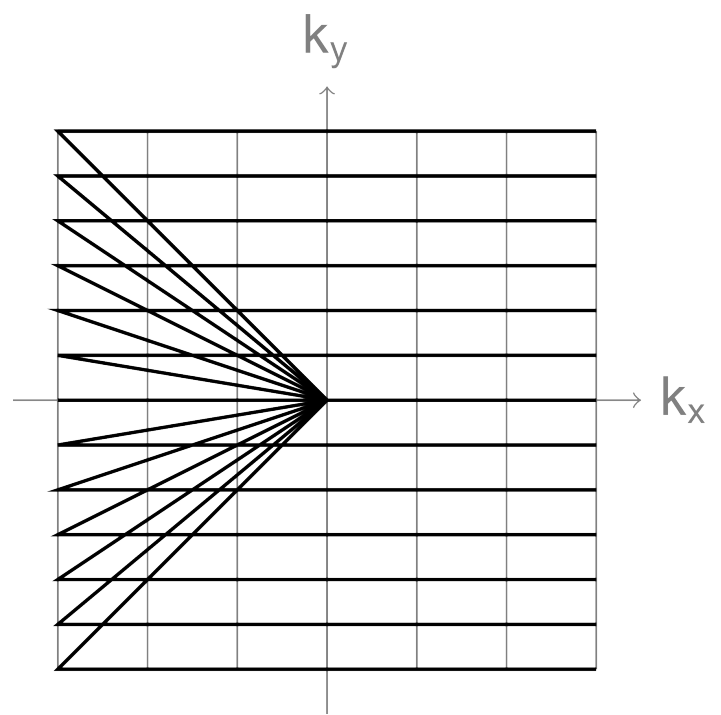

(a) Cartesian

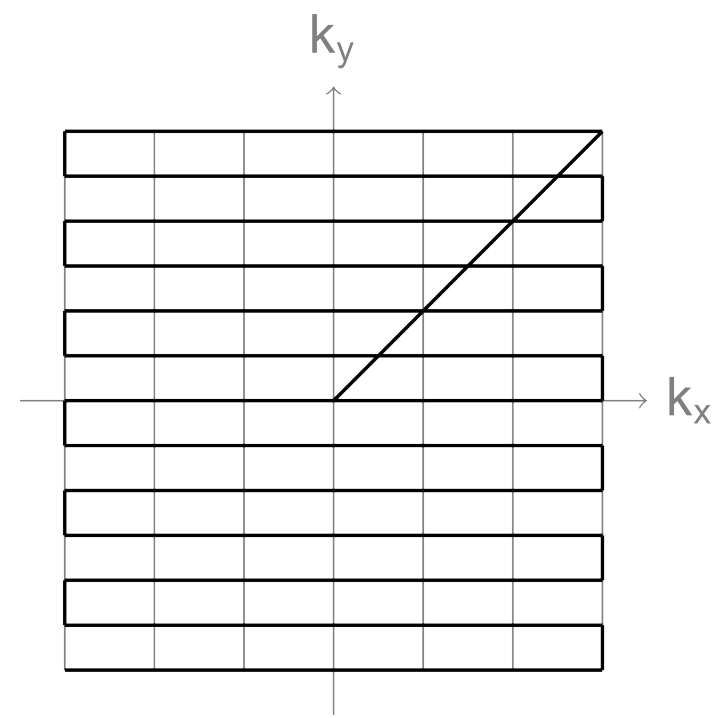

(c) Echo-planar

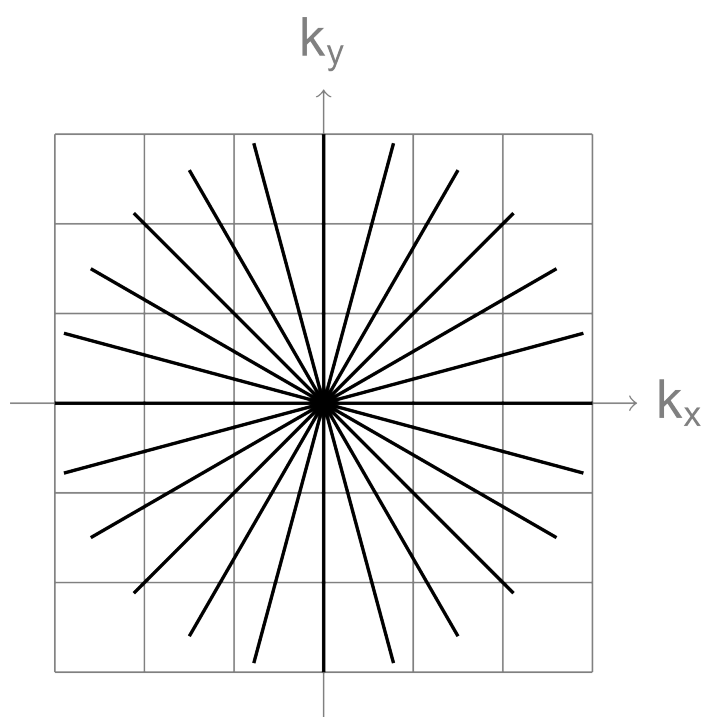

(b) Radial

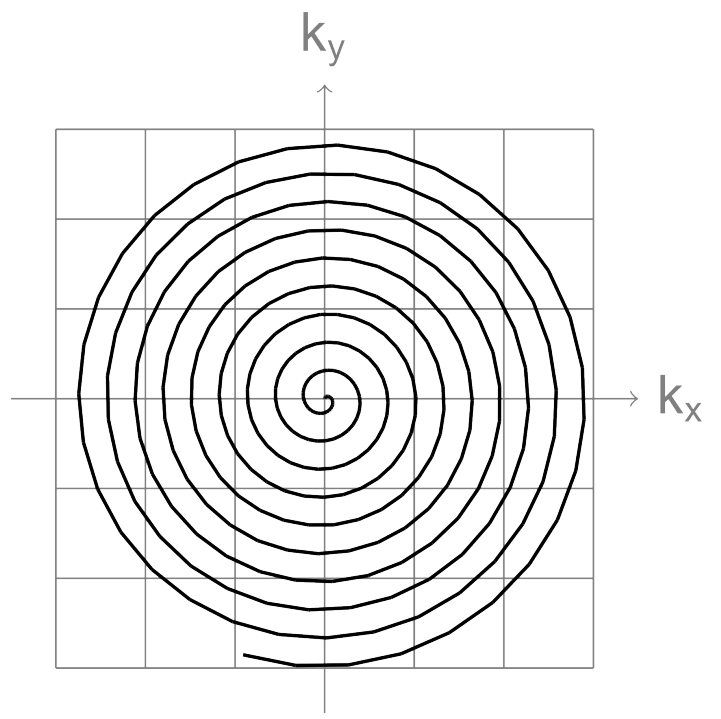

(d) Spiral

Figure 8 - Commonly used k-space trajectories in MRI [59]

In radial or cartesian trajectories, lines in k-space are subsequently sampled, for this purpose, a gradient scheme as shown in figure 3 is typically used: first, a pre-phaser gradient is applied to start the line at the edge of the sampled area in k-space, and sampling of a line is achieved by switching on a gradient in the opposite direction during signal readout. For radial trajectories, sampled lines are called spokes, and the spatial orientation of the pre-phaser and the gradient are adapted to the spoke orientations. For cartesian trajectories, a phase-encoding gradient is applied in addition to the pre-phaser prior to sampling of a line to obtain the correct position of the line. In radial or cartesian trajectories, one echo of one of the types described in section 2.1 .2 is usually generated for the readout of each line or spoke. 


\subsubsection{Echo-planar imaging}

By contrast, the echo-planar trajectory [43] is designed for the rapid acquisition of an MR image using only one RF-refocused echo, usually a spin echo. The entire two-dimensional k-space is sampled in one long trajectory using an alternating gradient in the direction of the cartesian lines for readout, as well as small gradient moments in the perpendicular ("phase-encoding") direction between the readout intervals to proceed from one line to the next. The major drawback of this trajectory is that it produces image artifacts in the presence of inhomogeneities of the static magnetic field, which can be caused by the imaging hardware or by an inhomogeneous magnetic susceptibility [32. This can be understood as follows: if an image voxel contains two compartments where the static magnetic field differs by $\Delta B_{0}$, the relative phase of the two isochromats representing these compartments change by $\Delta \varphi=\gamma \Delta B_{0} \Delta t$ over a time $\Delta t$. The time difference between the sampling times of two adjacent points in k-space is particularly large in the phase-encoding direction: $\Delta t$ includes the sampling time of an entire k-space line and the necessary times for gradient switching. Hence, the signals originating from the two compartments will be represented in the $\mathrm{k}$-space image with strongly different spatial frequencies in the phase-encoding direction, but only slightly different spatial frequencies in the readout direction. Spatial frequencies in the k-space image correspond to locations in image space. Therefore, local magnetic field inhomogeneities lead to a mislocalization of measured signal primarily in the phase-encoding direction. If these mislocalizations exceed the size of a voxel, they can lead to image distortions or local alterations of the image intensity in the vicinity of locations where the gradient of the static magnetic field is large, e.g. air-tissue boundaries. Susceptibility-related distortions were also observed in diffusionweighted EPI experiments performed for this thesis (see section 5) and their avoidance is the main motivation for the use of the single-shot STEAM sequence, where a stimulated echo is generated for each radial spoke.

\subsubsection{Chemical shift selective (CHESS) imaging}

The Larmor frequency of a proton spin is dependent on the chemical environment of the proton, i.e. the type of molecule and the position within the molecule which the hydrogen atom occupies. This is due the shielding effect of the electron configuration. This effect is quantified by chemical shift $\sigma$, and the local magnetic field which acts on a proton is $B_{l o c}=B_{0}(1-\sigma)$, where $B_{0}$ is the local magnetic field for a reference configuration. In human tissue, there is generally a high abundance of protons in water and fat. The chemical shift between fat and water is $3.35 \mathrm{ppm}$ [19], which leads to a Larmor frequency shift of $\Delta \nu=430 \mathrm{~Hz}$ at a static magnetic field of 3T. This induces a systematic error in frequency encoding, and if the frequency shift is in the same order of magnitude as the bandwidth per pixel, this leads to visible artifacts in the image. For

cartesian k-space trajectories, a mislocalization by $N=\frac{\Delta \nu}{B W}$ pixels in the readout direction leads to a ghosting artifact, for radial encoding, the effect will be visible as streaking artifacts. 
An effective method to avoid such artifacts without sacrificing SNR with an unnecessarily high receiver bandwidth is the use of a CHESS pulse [20]. This is a $90^{\circ}$ pulse with a frequency bandwidth which selectively excites the spins in the fat tissue, followed by a gradient to dephase the transverse magnetization. This depletes the longitudinal magnetization from the protons in fat, which only relaxes slowly on a time scale of T1. Thus, after the CHESS pulse, an imaging sequence can be run, and MR signal can be acquired with negligible contributions from fat tissue.

\subsection{Diffusion-weighted MRI}

Diffusion-weighted MRI is the acquisition of MR images with an image contrast influenced by incoherent random motion of spins due to self diffusion of water molecules [41, 44, 56]. For diffusion-weighted MRI, a multitude of different image acquisition techniques have recently been proposed, which differ in the methods for creating an MRI signal which is sensitive to diffusion and in the k-space trajectory for signal readout [70, 49]. In addition, advanced mathematical modeling methods for diffusion processes in tissue are a vibrant and expanding field of research which aims to further develop the examination of tissue microstructure by MRI [1, 31, 51]. This section is limited to a description of the most basic model of directional diffusion in human tissue, the diffusion tensor, and its spatially resolved measurement using diffusion-weighted spin echoes as proposed by Stejskal and Tanner [52].

\subsubsection{Diffusion in human tissue}

Diffusion describes random motion of molecules. The degree of diffusion is generally quantified by the diffusion coefficient $D$. This can be most clearly understood in terms of the EinsteinSmoluchowski equation [11]: In 3 dimensions, the relation

$$
\left\langle r^{2}(t)\right\rangle=6 D t
$$

holds, where $r(t)$ is the distance of a particle to its original position after a diffusion time $t$. In human tissue, the mean squared displacement of water molecules on time scales of tens of milliseconds is primarily determined by the local cellular structure of the tissue. Hence, in a large ventricle filled mainly with water, the diffusion coefficient is higher than in tissues with densely packed cells, where water molecules are repelled multiple times, which reduces their total displacement. Pathological changes in the cellular structure can alter the diffusion coefficient, hence, diffusion-weighted MRI has become an important tool for cancer diagnosis [58]. For anisotropic structures, e.g. nerve fibers or muscle fibers, it is useful to describe diffusion of water molecules with the diffusion tensor $\underline{D}$, which can be represented as a positive definite symmetric $3 \times 3$ matrix. In this model, the coefficient of diffusion in any direction described by a unit vector $\vec{n}$ is $\vec{n}^{T} \underline{D} \vec{n}$. For a concentration $c$ of diffusing particles, conservation of mass yields the diffusion equation, also 
known as Fick's second equation:

$$
\frac{\partial c}{\partial t}=\nabla \cdot(\underline{D} \nabla c)
$$

\subsubsection{The diffusion-weighted spin echo}

The extension of the Bloch equation (equation 1) to include the effect of diffusion on the dynamics of the magnetization follows from equation 5 and the fact that the magnetization is proportional to the concentration of protons. This leads to the Bloch-Torrey equation [57]:

$$
\frac{d \vec{M}}{d t}=\gamma \vec{M} \times \vec{B}+\left(\begin{array}{c}
-\frac{M_{x}}{T 2} \\
-\frac{M_{y}}{T 2} \\
\frac{M_{0}-M_{Z}}{T 1}
\end{array}\right)+\nabla \cdot(\underline{D} \nabla \vec{M})
$$

where the gradient operator acts on the individual coordinates of the magnetization vector. For the measurement of the diffusion coefficient using nuclear magnetic resonance, Stejskal and Tanner [52] proposed a spin-echo sequence as shown in figure 9, with two identical gradient pulses, one before and one after the refocusing $180^{\circ}$ pulse.

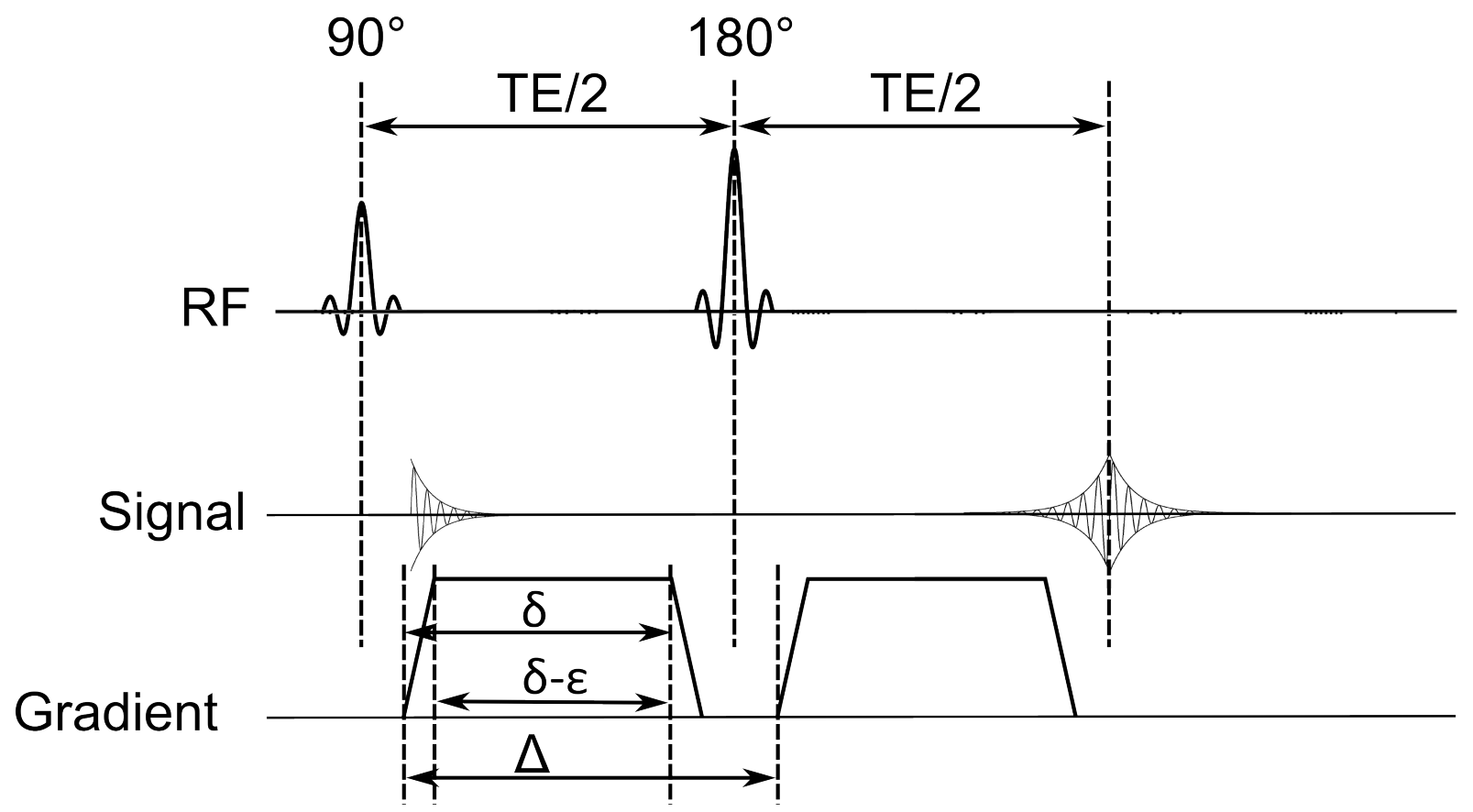

Figure 9 - The diffusion-weighted spin echo. A strong gradient is switched on before and after the $180^{\circ}$ pulse. This leads to a dephasing effect in the presence of incoherent motion along the direction of the gradient.

The solution of the Bloch-Torrey equation without the relaxation term for the trapezoidal gradients as depicted in figure 9 [3, 52] yields the signal strength: 


$$
S=S_{0} e^{-b \vec{g}^{T} \underline{D} \vec{g}}
$$

with $S_{0}$ being the signal strength without the presence of diffusion gradients, the unit vector in the direction of the diffusion gradient $\vec{g}$ and the b-value

$$
b=(\gamma G)^{2}\left[\delta^{2}\left(\Delta-\frac{\delta}{3}\right)+\frac{\epsilon^{3}}{30}+\frac{\delta \epsilon^{2}}{6}\right] .
$$

$G$ is the amplitude of the diffusion gradient. Intuitively, the dependence of the signal on the diffusion coefficient can be understood by first considering the case of stationary spins. The effect of the first diffusion gradient on the phase of the spins is completely reverted by the second gradient, and complete refocusing of the spin echo takes place. For the case of diffusion, i.e. incoherent random motion of spins along the gradient direction, the resulting phase of the spins will depend on their individual trajectories of motion while the gradients are switched on. Therefore, refocusing is incomplete, and the larger the degree of random motion is, the more dephasing will be caused by the diffusion gradients. On the other hand, coherent motion of tissue volumes larger than a voxel does not lead to dephasing and signal loss, but to a constant phase in the magnetization

$$
\varphi=\gamma \int_{t_{1}}^{t_{2}} \vec{G}(t) \cdot \vec{x}(t) d t-\gamma \int_{t_{3}}^{t_{4}} \vec{G}(t) \cdot \vec{x}(t) d t
$$

with $t_{1}$ and $t_{2}$ being the start and end time of the first diffusion gradient, and $t_{3}$ and $t_{4}$ the start and end time of the second diffusion gradient.

\subsubsection{MRI measurements of the diffusion tensor}

To measure the diffusion tensor

$$
\underline{D}=\left(\begin{array}{ccc}
D_{x x} & D_{x y} & D_{x z} \\
D_{x y} & D_{y y} & D_{y z} \\
D_{x z} & D_{y z} & D_{z z}
\end{array}\right)
$$

it is necessary to measure the diffusion-weighted signal for one b-value with at least 6 gradient directions, as well as the non-diffusion-weighted signal. Setting up equation 6 for all gradient directions and taking the logarithm of both sides of the equation yields a linear inverse problem with the 6 parameters defining the diffusion tensor as unknowns. For measurements with a higher number of gradient directions or multiple b-values, the diffusion tensor can be calculated using a weighted linear least squares algorithm. These and more advanced methods for diffusion tensor calculation have been summarized by Kingsley [37]. The two most important metrics for the diffusion tensor are the apparent diffusion coefficient (ADC) and the fractional anisotropy (FA). The ADC is the mean eigenvalue of the diffusion tensor and can be used as a simple scalar measure 
to describe the degree to which diffusion in tissue is hindered, e.g. by cell membranes. The FA is a measure to describe the degree of directionality of these barriers and is defined as

$$
F A=\sqrt{\frac{3}{2} \frac{\sum_{i=1}^{3}\left(\lambda_{i}-A D C\right)^{2}}{\sum_{i=1}^{3} \lambda_{i}^{2}}}
$$

with $\lambda_{i=1,2,3}$ the eigenvalues of the diffusion tensor. Besides maps of the ADC and the FA, diffusion-weighted MR images can be directly used for clinical diagnosis (e.g. [36]). Diagnostic images can be obtained either by averaging over the magnitudes of the image intensities acquired with the different gradient directions, or by computing the trace-weighted image $I=S_{0} e^{-\frac{b}{3} \operatorname{tr}(D)}$. When directionality of diffusion is of no interest, diffusion-weighted imaging can also be limited to three orthogonal gradient directions. The trace-weighted image $\operatorname{Tr} a(b)$ is then calculated as the geometric mean of the images with the respective b-value, and the ADC is calculated from the model $\operatorname{Tr} a(b) \sim e^{-b \cdot A D C}$

The diffusion-weighted imaging sequence which is routinely used in clinical practice reads out a diffusion-weighted (DW) spin echo with an echo-planar k-space trajectory and is therefore called diffusion-weighted echo-planar imaging (DW-EPI) [41]. This allows fast imaging with a high signalto-noise ratio due to the direct and rapid readout of the diffusion-weighted spin echo, but has the disadvantage a high sensitivity to susceptibility-related artifacts as discussed in section 2.1.3.

\subsection{Image reconstruction by nonlinear inversion}

In parallel imaging, multiple receive coils are used for data acquisition. The signal measured in each coil is influenced by its spatial sensitivity profile. These sensitivities depend on the geometry and location of the coils, but also on the distribution of the magnetic susceptibility in the body in the MRI device. Figure 10 shows an example of MR images reconstructed from signals acquired with four coil elements placed around a phantom.
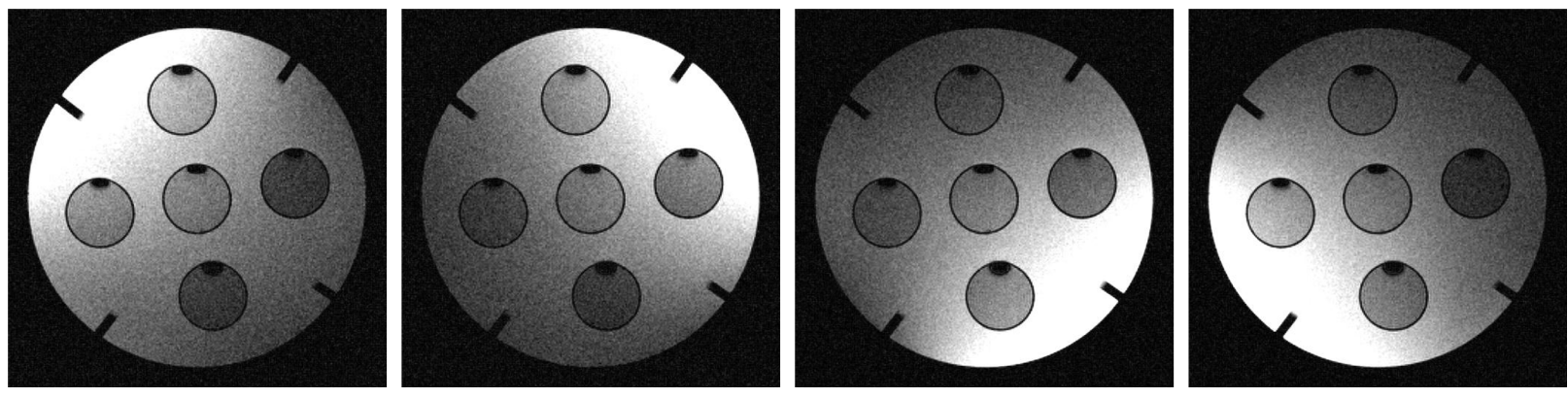

Figure 10 - Images reconstructed from the data acquired using four different receive coils using a direct Fourier transform [59]. 
For a coil element denoted by the index $\mathrm{j}$ with the spatial sensitivity profile $c_{j}(\vec{x})$, equation 3 which describes the relation between the measured signal and the transverse magnetization becomes:

$$
S_{j}(t)=\int c_{j}(\vec{x}) M_{1}(\vec{x}, t) e^{i \vec{k}(t) \cdot \vec{x}} d V
$$

Hence, the image reconstructed from the data acquired in a single coil element by a direct Fourier transform is the product of the distribution of transverse magnetization and the sensitivity profile. In 2008, Uecker et al. proposed the formulation of the image reconstruction problem in parallel imaging as a nonlinear inverse problem and its solution by means of the iteratively regularized Gauss-Newton method [60]. This algorithm of nonlinear inversion (NLINV), as well as its extension to radial trajectories [62] are the basis of the image reconstruction methods described in this thesis and shall be summarized in this section.

The unknown image content $\rho$ and coil sensitivities $c_{1,2, \ldots, n}$ for $\mathrm{n}$ coils are represented as

$$
x=\left(\begin{array}{c}
\rho \\
c_{1} \\
\cdots \\
c_{n}
\end{array}\right) .
$$

Parallel MRI is modeled according to equation 7; the measured data $y=F(x)$ with

$$
F: x \mapsto\left(\begin{array}{c}
P_{k} \mathcal{F} P_{F O V} c_{1} \rho \\
\ldots \\
P_{k} \mathcal{F} P_{F O V} c_{n} \rho
\end{array}\right)
$$

with a point-wise multiplication between the image content and the respective coil sensitivities, $P_{F O V}$ denoting the projection onto a compact field of view, and $P_{k}$ the projection onto the sampled k-space trajectory. For the purpose of imposing a large degree of smoothness on the coil sensitivities, a preconditioning operator $W$ is included in the reconstruction algorithm: $x=W \hat{x}$ with $\rho=\hat{\rho}$ and $\hat{c}_{j}=\left(1+s\|\vec{k}\|^{2}\right)^{l / 2} \mathcal{F} c_{j}$. The forward operator is transformed to express the measured data in terms of $\hat{x}$ :

$$
y=F(x)=F \circ W(\hat{x})=G(\hat{x})
$$

This nonlinear inverse problem is solved in multiple Newton iterations. The m-th Newton iteration (starting with 0) solves the problem after linearization around the current estimate $\hat{x}_{m}$ :

$$
\hat{x}_{m+1}=\hat{x}_{m}+\underset{d \hat{x}}{\operatorname{argmin}}\left[\left\|D G\left(\hat{x}_{m}\right) d \hat{x}-\left(y-G\left(\hat{x}_{m}\right)\right)\right\|^{2}-\alpha_{m}\left\|\hat{x}_{m}+d \hat{x}-\hat{x}_{0}\right\|^{2}\right] .
$$

$D G$ denotes the Fréchet derivative of $G$. The regularization parameter $\alpha_{m}$ is defined by a 
decreasing sequence of positive numbers, for the computations described in this thesis $\alpha_{m}=0.5^{m}$. The regularization term in equation 8 describes a strong penalization of high spatial frequencies in the coil sensitivities for the parameters $s=100$ and $l=32$, which are used for all NLINV reconstructions described in this thesis. This shows that the preconditioning operator $W$ enforces the reconstruction of very smooth coil profiles. From equation 8 , the update rule follows:

$$
\hat{x}_{m+1}-\hat{x}_{m}=\left(D G^{H}\left(\hat{x}_{m}\right) D G\left(\hat{x}_{m}\right)+\alpha_{m} I\right)^{-1}\left(D G^{H}\left(\hat{x}_{m}\right)\left(y-G\left(\hat{x}_{m}\right)\right)-\alpha_{m} \hat{x}_{m}\right) .
$$

The inversion of the first factor on the right hand side is computed using conjugate gradients 23.

For a cartesian sampling trajectory, the implementation of $P_{k} \mathcal{F} P_{F O V}$ is trivial, because the data points are already on a cartesian grid, allowing direct computations of fast Fourier transforms which automatically yields images on the compact domain of the field of view. The extension to radial trajectories is detailed in ref. [62]. The projection onto the field of view is achieved by interpolating the radially sampled data onto a 2-fold oversampled cartesian grid prior to image reconstruction. Furthermore, the calculation of the updated estimate for each Newton iteration involves convolutions with the point spread function which is defined by the sampling trajectory.

In practice, image reconstruction by nonlinear inversion from radially sampled data [62, 63. includes the following computation steps: First, the amount of data to be processed is reduced to a predefined number of virtual receive channels by principal-components-based data compression. Then, the data are corrected for delays of the imaging gradients [48, 64], interpolated onto a cartesian grid using Kaiser-Bessel functions, and the point-spread function is computed from the known k-space trajectory. Iterative image reconstruction is performed on the resulting k-space images.

\subsection{Use of resources}

For the development and application of the image acquisition and reconstruction techniques developed in this thesis, the following resources were used:

- All MR experiments were carried out on a 3T Prisma MRI device, Siemens, Erlangen

- For brain imaging, a 64-channel head coil was used

- For cardiac and prostate imaging, the combination of a body-array coil and a spine coil were used

- The described imaging sequences were implemented in $\mathrm{C}++$ using the Siemens IDEA VD13D sequence development framework. These sequences use code from a radial fast low-angle shot (FLASH) sequence [21] implemented by Dirk Voit in the Biomedizinische NMR Forschungs GmbH, Göttingen. 
- Image reconstruction algorithms were implemented in Matlab (R2015b; The MathWorks, Inc, Natick, MA). These algorithms partly use an interface with a graphics processing unit (GeForce GTX TITAN; NVIDIA, Santa Clara, CA) provided by the MATLAB Parallel Computing Toolbox. The software which was developed for image reconstruction includes source code from previous developments for data preprocessing, NLINV, and image denoising by the Biomedizinische NMR Forschungs GmbH which have been mainly used for real-time MRI. 


\section{The undersampled radial STEAM sequence}

\subsection{Sequence design}

\subsubsection{From STEAM to single-shot STEAM}

The single-shot STEAM sequence was initially developed by Frahm et al. in 1985 [16]. It is obtained by replacing the third $90^{\circ}$ pulse of the basic STEAM sequence (figure 11a) by a series of pulses with smaller flip angles (the "readout train"), as shown in figure 11b.

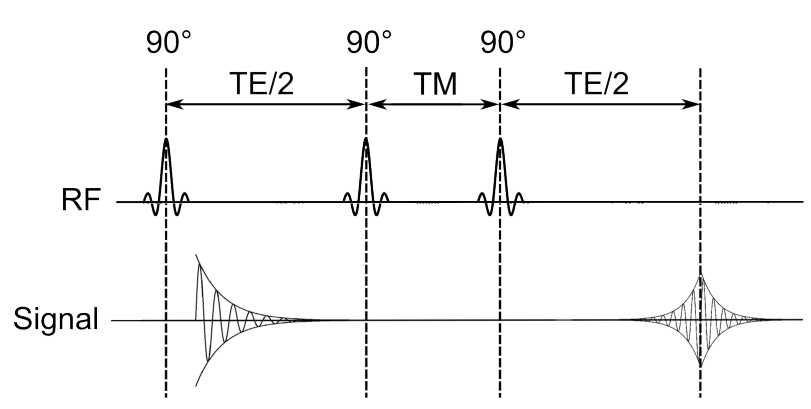

(a) Basic STEAM sequence

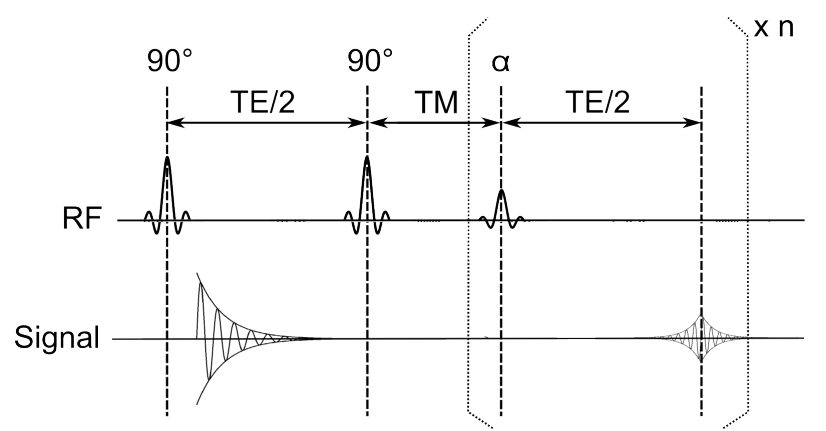

(b) Single-shot STEAM sequence

Figure 11 - The single-shot STEAM sequence: n stimulated echoes are generated with one excitation using a train of small flip angle pulses.

After the first two $90^{\circ}$ pulses, the distribution of spins dephases into a state with the excited magnetization coherently stored in the longitudinal direction (see section 2.1.2, figure $7 \mathrm{f}$ ). This prepared magnetization decays on a time scale of $\mathrm{T} 1$, which is in the order of $1 \mathrm{~s}$ in the human body. Therefore, a third radiofrequency pulse will still induce a stimulated echo with significant signal strength even if it is applied after a waiting time in the order of $0.1-1 \mathrm{~s}$ after the second $90^{\circ}$ pulse.

The single-shot STEAM sequence takes advantage of this feature. Instead of fully depleting the magnetization with one $90^{\circ}$ pulse, the stored magnetization is depleted step-wise with low flip angle pulses ( $\alpha$ pulses), and each of these pulses generates a stimulated echo. This can be seen as a distribution of the stored magnetization among the stimulated echoes of the readout train, with only moderate signal decay due to $\mathrm{T} 1$ relaxation. As for the basic STEAM sequence, the condition for "clean" stimulated echoes without spurious signal is that the transverse magnetization is fully dephased before the second $90^{\circ}$ pulse, and before each $\alpha$ pulse. Moreover, the FID signal from the $\alpha$ pulses must be dephased to avoid a superposition of the stimulated echo with a spurious gradient echo.

Each of the stimulated echoes is spatially encoded as a cartesian line or a radial spoke. Unlike gradient-echo sequences, particularly those using echo-planar signal readout, this sequence does not lead to susceptibility-induced image artifacts, because at the time point of the stimulated echo, 
when signal readout takes place, spins with different Larmor frequencies due to local magnetic susceptibilities are rephased. A further difference in comparison to most other MRI sequences, e.g. FLASH, is that undersampling does not lead to a lower, but to a higher signal-to-noise ratio. If the number of stimulated echoes to be generated is reduced, higher flip angles can be applied to deplete larger fractions of the stored magnetization for each stimulated echo, so a higher SNR per sampled line compensates the lower total number of lines. Additionally, the effect of T1 decay is weaker for shorter readout trains.

The possibility of achieving high undersampling factors with radial k-space trajectories in combination with iterative image reconstruction motivated the initial development of the radial single-shot STEAM sequence [6]. The following subsections describe a new implementation of this sequence for this work. First, an overview of the sequence is given, followed by a description of two modifications which were introduced to remove image artifacts.

\subsubsection{The radial single-shot STEAM sequence}

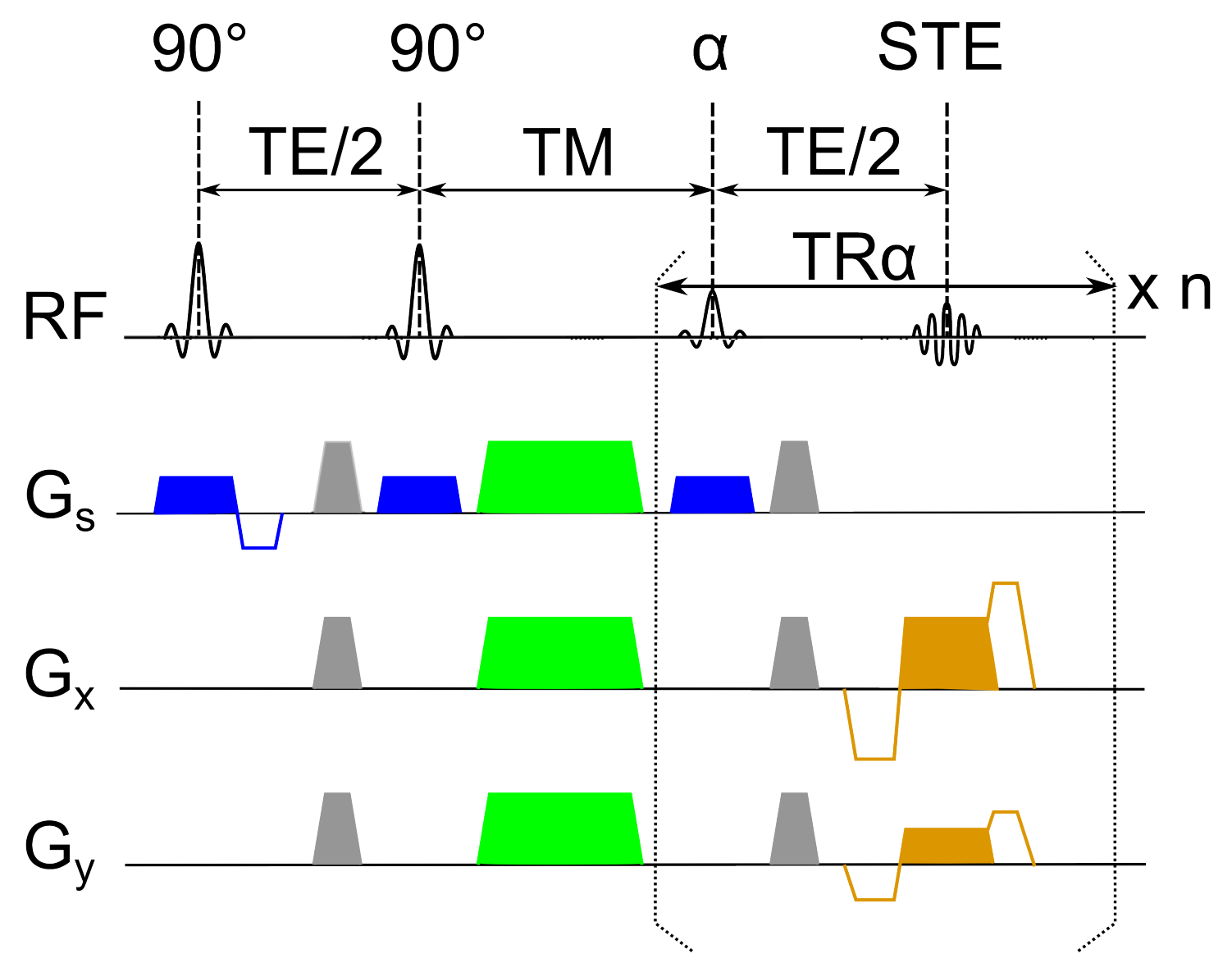

Figure 12 - The radial single-shot STEAM sequence.

Figure 12 shows the complete single-shot STEAM sequence with radial encoding, with the radiofrequency pulses as well as the gradients in the slice direction, $G_{s}$ and in the two in-plane 
directions, $G_{x}$ and $G_{y}$. The transverse magnetization is dephased, or "spoiled", with the gradients shown in green and gray before the second $90^{\circ}$ pulse, before the start of the readout train, and after each $\alpha$ pulse. The spoiler gradient before the second $90^{\circ}$ pulse must have the same gradient moment (time integral) as the FID spoiler, i.e. the gradient following the $\alpha$ pulses shown in gray, to rephase the transverse magnetization for the stimulated echoes.

Excitation of the desired tissue slice is achieved with the gradients shown in blue. The gradient directly following the slice-selection gradient for the first $90^{\circ}$ pulse (blue outline) compensates the dephasing effect of the slice-selection gradient. The readout gradient shown in orange encodes the stimulated echoes as radial spokes in $\mathrm{k}$-space. This gradient is preceded by a pre-phaser (orange outline in figure 12 which ensures that the peak of the stimulated echo is aligned with the k-space center. The readout gradient is directly followed by a spoiler gradient (orange outline), which dephases the remaining transverse magnetization to avoid any spurious signal from one stimulated echo in the acquisition of the subsequent radial spokes. The orientation of the pre-phaser, the readout gradient, and the spoiler following the readout gradient (all shown in orange) are adapted to the spoke angle $\theta$ to the $\mathrm{x}$ axis, i.e., with $G_{x}=G \cos (\theta)$ and $G_{y}=G \sin (\theta)$, $\mathrm{G}$ denotes the respective gradient amplitudes, which are positive for the readout gradient and the spoiler, and negative for the pre-phaser. The timing of the sequence is set by the stimulated echo time TE and the readout repetition time $\operatorname{TR} \alpha$. The time between the second $90^{\circ}$ pulse and the first low-angle pulse of the readout train is the mixing time TM.

This radial single-shot STEAM sequence contains two modifications compared to the initial sequence by Block and Frahm [6]. Firstly, the calculation of variable flip angles was modified to achieve a sufficiently homogeneous distribution of signal strength for the radial spokes to avoid streaking artifacts. Secondly, the spoiling method for the readout train was modified to more effectively suppress signal contributions from gradient echoes.

\subsubsection{Variable flip angle scheme}

Methods The signal strength for each stimulated echo, i.e. each radial spoke in the acquired data, depends on the respective flip angle as well as the remainder of the longitudinally stored magnetization before the RF pulse. Hence, to compensate for the step-wise depletion of the stored magnetization during readout, the first echoes must be generated with lower flip angles than the later echoes. The signal strength $S_{i}$ for the $\mathrm{i}$-th echo is given by $S_{i}=M_{i} \sin \left(\alpha_{i}\right)$, with $\alpha_{i}$ the flip angle of the $\mathrm{i}$-th pulse of the readout train and $M_{i}$ the magnetization before that pulse. Each readout pulse reduces the magnetization by a factor $\cos \left(\alpha_{i}\right)$, in addition, T1-relaxation must be taken into account. Thus,

$$
M_{i+1}=M_{i} \cos \left(\alpha_{i}\right) e^{-\frac{T R \alpha}{T 1}}
$$

To achieve equal signal strength for all stimulated echoes, the condition $S_{i}=S_{i+1}$ is set for 
$i=1, \ldots, n-1$ with a total number $\mathrm{n}$ of stimulated echoes. This yields the recursive formula

$$
\alpha_{i}=\arctan \left(\sin \left(\alpha_{i+1}\right) e^{-\frac{T R \alpha}{T 1}}\right) .
$$

This variable flip angle model has been developed previously [14, 33], with $\alpha_{n}=90^{\circ}$ to take advantage of the remaining magnetization and an a priori estimate of $\mathrm{T} 1$, which is determined by the anatomical location.

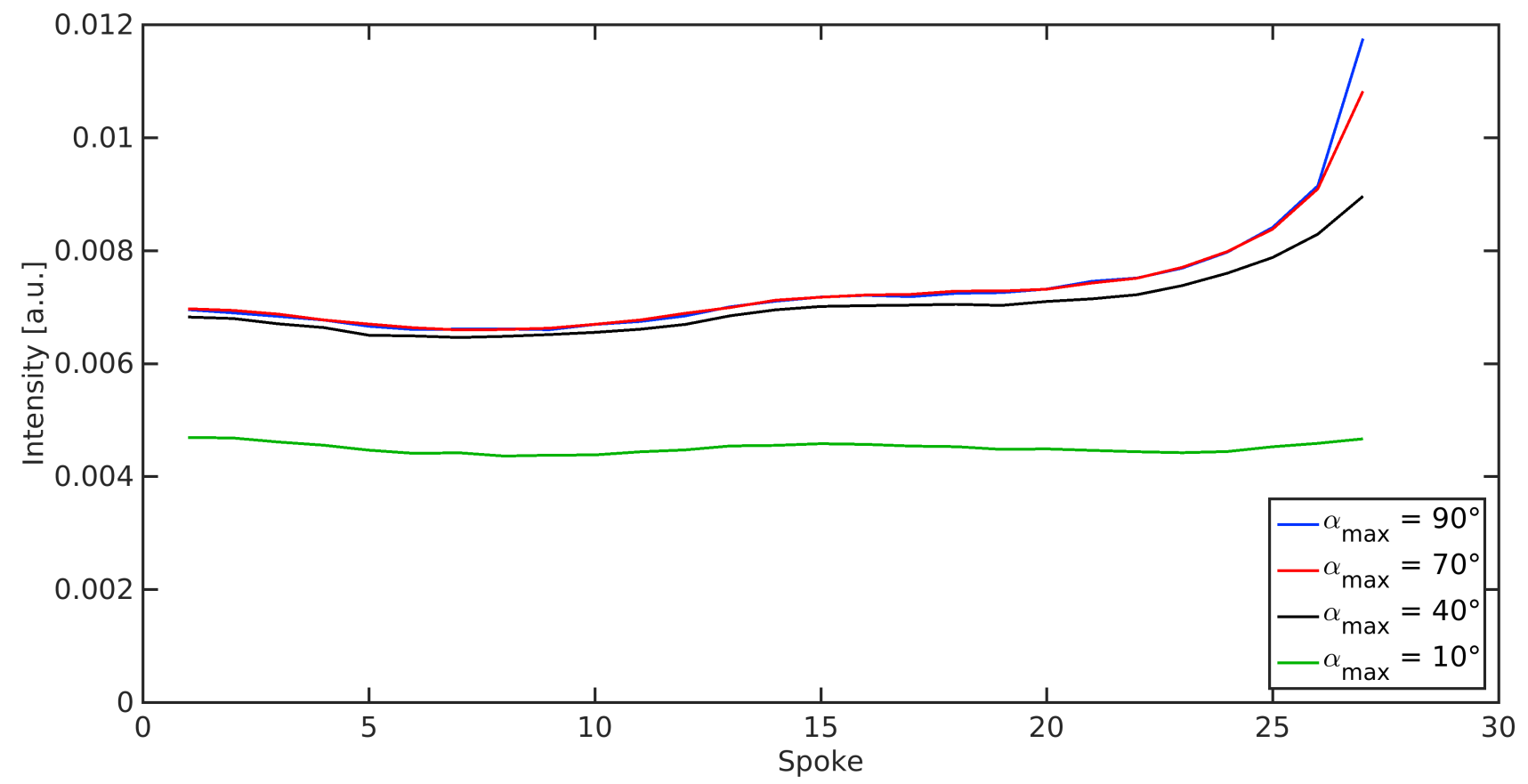

(a) Total signal strength (root-sum-squares over sampled data points and imaging channels) in the raw data for 27 spokes ordered by the time of acquisition and four different maximal flip angles

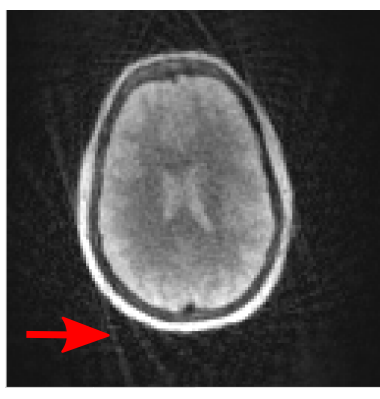

(b) $\alpha_{\max }=90^{\circ}$

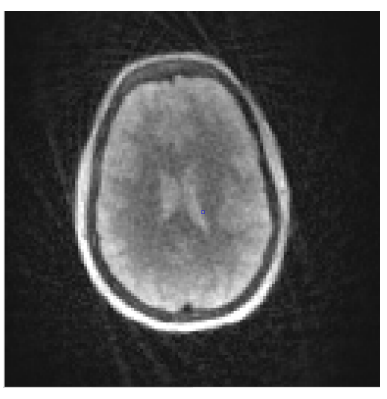

(c) $\alpha_{\max }=70^{\circ}$

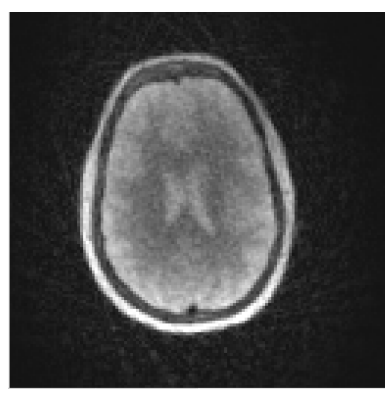

(d) $\alpha_{\max }=40^{\circ}$

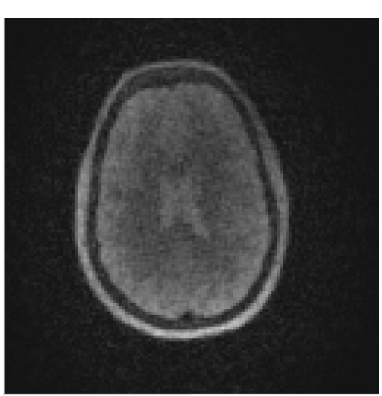

(e) $\alpha_{\max }=10^{\circ}$

Figure 13 - Effect of the maximum flip angle on streaking artifacts (red arrow) and signal loss in a single-shot STEAM imaging of the brain. The images in the lower row represent NLINV reconstructions from the same datasets used for the calculation of signal strengths in the raw data as shown in figure $13 \mathrm{a}$.

In practice, deviations of the actual flip angle from the nominal flip angle as defined by the radiofrequency pulses alter the signal strengths. These deviations are caused by inhomogeneities of the static magnetic field, off-resonance excitation, and the inhomogeneous intensity of the RF 
amplitude across the width of the excited slice due to the shape of the RF pulse [65. The actual flip angle is typically significantly lower than the nominal flip angle [2], with relative deviations of more than 10\%. Therefore, the flip angle model systematically underestimates the remaining magnetization before the pulses in the later part of the readout train and generates a higher signal intensity in the last few spokes than in the other spokes.

This problem was solved heuristically, by introducing the variable parameter $\alpha_{\max }=\alpha_{n}<90^{\circ}$, which dampens the hyperintensity in the last spokes.

Results Figure $13 \mathrm{a}$ shows the effect of the maximal flip angle $\alpha_{\max }$ on the signal strength in the 27 spokes as well as the image quality for a brain scan performed with the radial singleshot STEAM sequence. Reducing $\alpha_{\max }$ from $90^{\circ}$ to $40^{\circ}$ strongly improves the signal homogeneity between the 27 spokes with minimal signal loss, whereas $\alpha_{\max }=10^{\circ}$ reduces the signal strength in all spokes by approximately one third. NLINV reconstructions of the corresponding datasets (figure 13) show that the directional streakings at the edge of the brain, which are caused by the hyperintense radial spokes for $\alpha_{\max }=90^{\circ}$ are much weaker at $\alpha_{\max }=40^{\circ}$ with no other change in image quality, and a clearly lower SNR for $\alpha_{\max }=10^{\circ}$.

\subsubsection{Spoiling in the readout train}

Methods The method used for handling the transverse magnetization in the readout train differs from the method previously proposed for radial single-shot STEAM MRI [6] as shown in figure 14. Instead of applying a rewinder gradient anti-parallel to the readout gradient to rephase the transverse magnetization, a spoiler gradient is applied in the same direction as the readout gradient to dephase the remaining transverse magnetization.

The effect of the new spoiling method on in-vivo image quality was tested with two experiments on the brain of a volunteer. First, the readout train of the single-shot STEAM sequence was run without preparation of the magnetization to identify signal from spurious gradient echoes. This experiment was executed with a rewinder and with a spoiler gradient, respectively. Second, the entire sequence was run with both settings to investigate the effect of the spoiling method on the image quality. Images were reconstructed using NLINV.

Results The results of these experiments are shown in figure 15. The raw data acquired by running only the readout train without excitation pulses show a significant signal intensity which strongly increases towards the end of the readout train if a rewinder gradient is used. By contrast, the readout train run with spoiler gradient yields no significant signal intensity. This shows that the spoiler gradient effectively suppresses gradient echoes which may contaminate the acquired signal if a rewinder gradient is used. The in-vivo images of the brain acquired with the two settings show generally similar image quality, but a locally lower intensity for the image acquired with the rewinder gradient in the upper-right region of the brain (red circles in figure 15). 


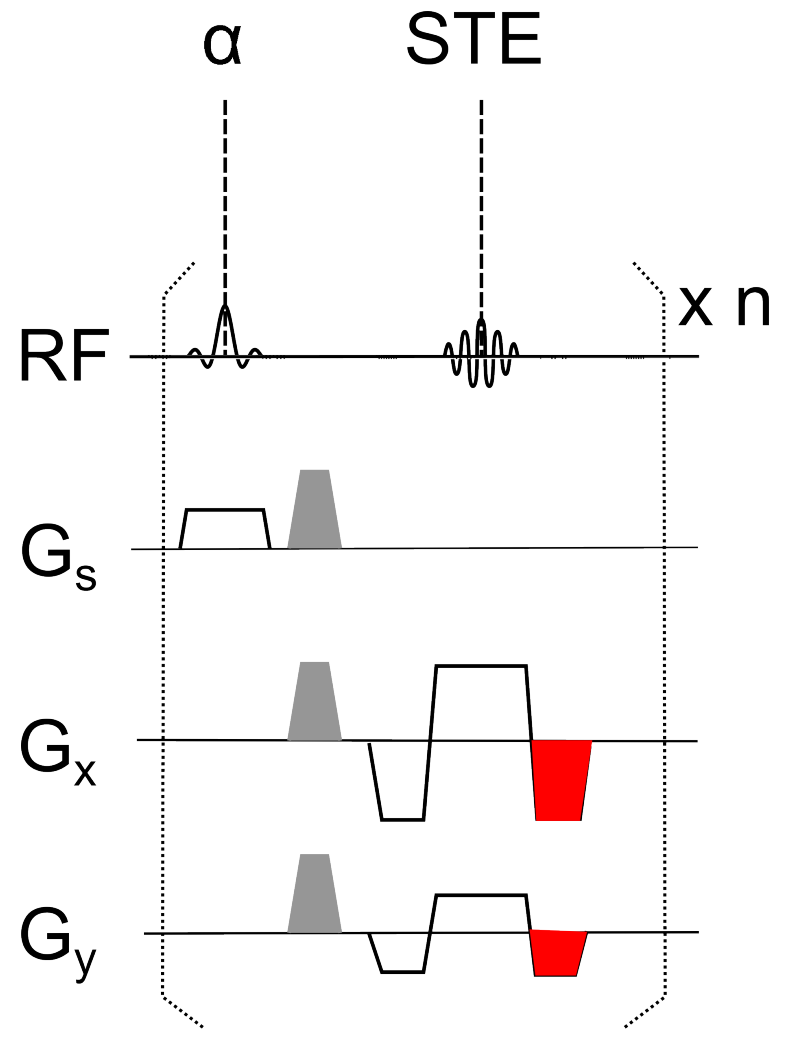

(a) Rewinder

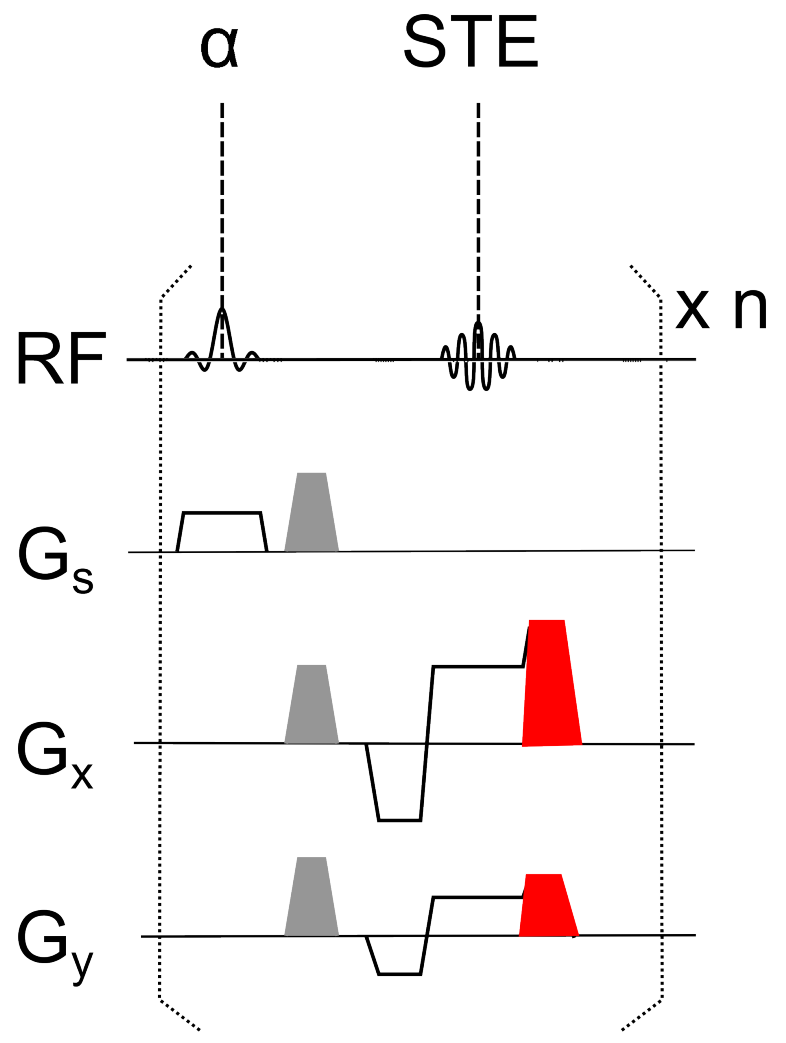

(b) Spoiler

Figure 14 - The readout train of the single-shot STEAM sequence with a rewinder gradient [6] and a spoiling gradient. 


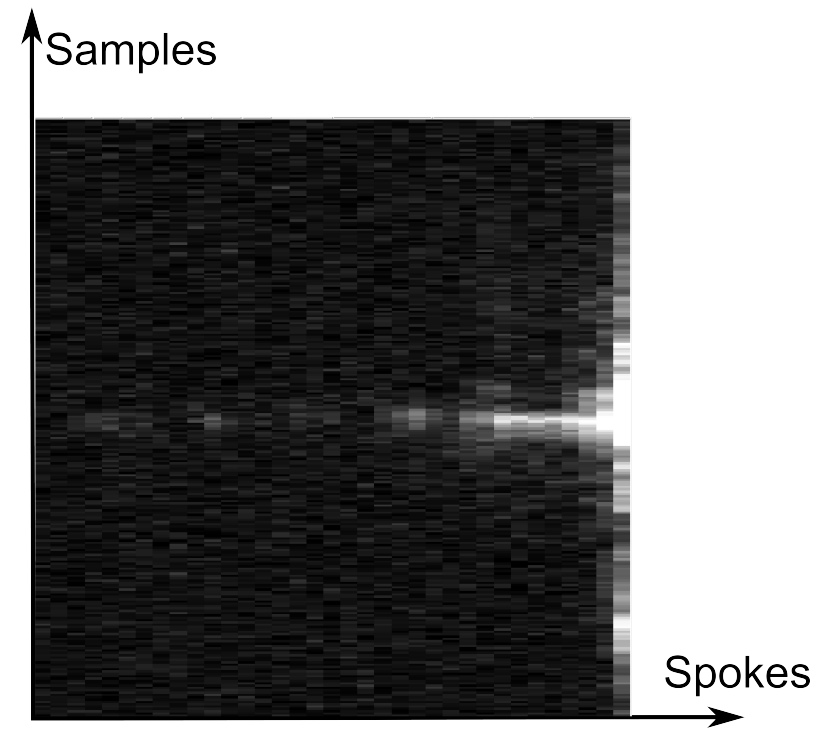

(a) Rewinder

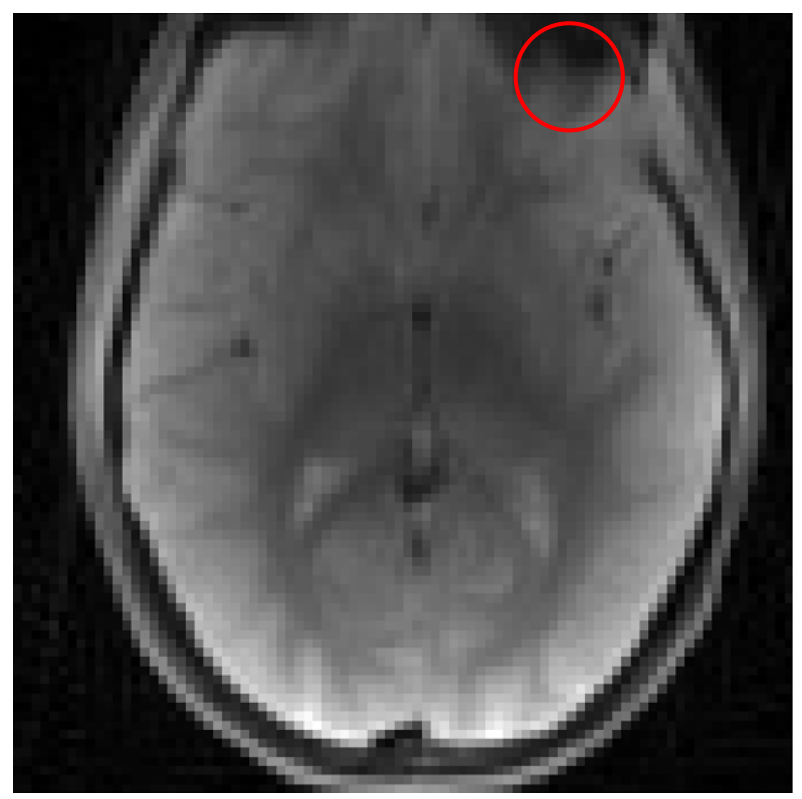

(c) Rewinder

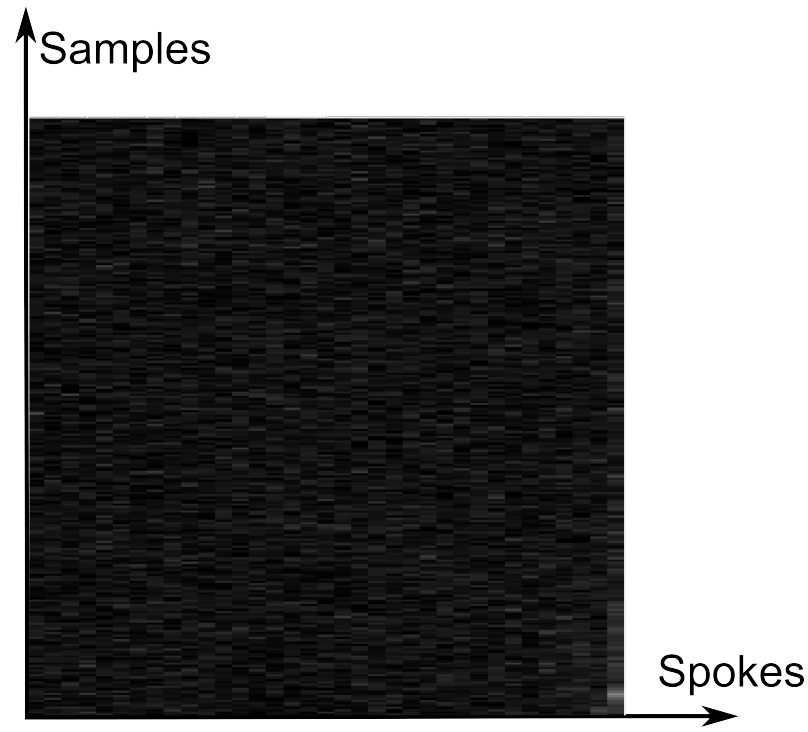

(b) Spoiler

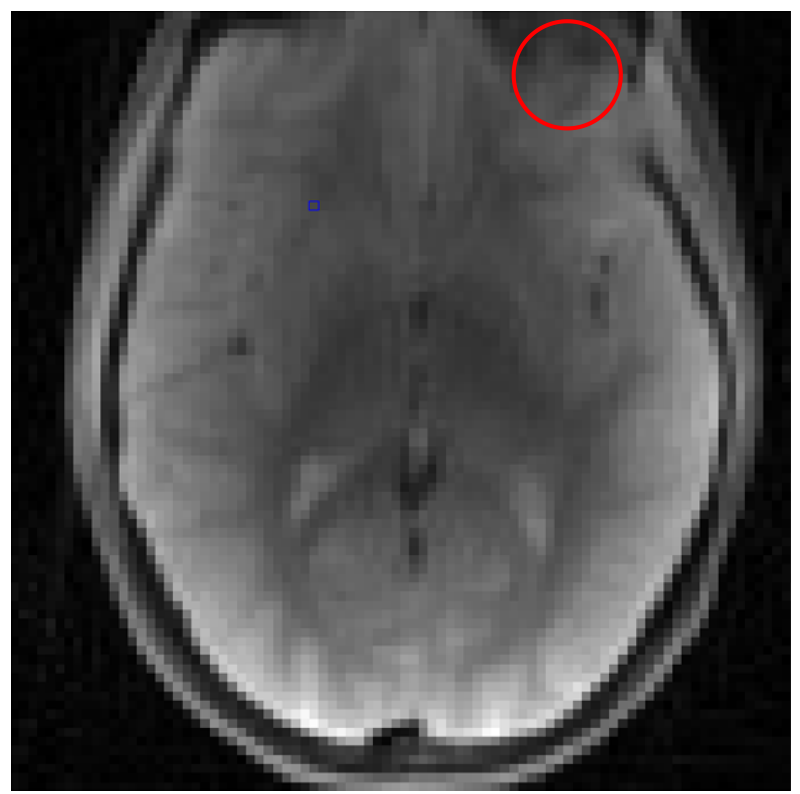

(d) Spoiler

Figure 15 - The effect of a rewinder gradient vs. a spoiler gradient after the readout pulse on in-vivo brain images. Upper row: The raw data (root-sum-squares over all imaging channels) acquired by running only the readout train of the STEAM sequence with (a) a rewinder gradient and (b) a spoiler gradient after the readout pulse. The brightness represents the intensity in the data points of the radial spokes ordered from left to right according to the time of signal readout. (c) and (d): Brain images reconstructed after data acquisition with the single-shot STEAM sequence with (c) a rewinder and (d) a spoiler gradient. In the region encircled in red, lower intensities are observed when a rewinder gradient is switched. 


\subsubsection{Discussion}

The proposed modifications of the radial single-shot STEAM sequence substantially improve the performance of the sequence with regard to the suppression of image artifacts. In particular, the modified variable flip angle model visibly suppresses streaking artifacts at a minimal cost in SNR. This is a viable, and probably favorable alternative to the approach presented in the initial publication on radial single-shot STEAM MRI [6], which merely suggests temporal reorderings of radial spoke angles to distribute the hyperintensities across a wider angular range, but does nothing to reduce them.

The experiment on the modified spoiling of the transverse magnetization in the readout train shows that with the new spoiling method, the train of $\alpha$ pulses does not generate any spurious MR signal which would be superimposed with the desired stimulated echoes. This indicates that the spoiler effectively dephases the transverse magnetization. By contrast, the use of a rewinder gradient leads to significant signal strength which gradually emerges and becomes stronger towards the end of the readout train. This suggests that the observed signal is produced by the interaction of spins with multiple RF pulses of the gradient echo train, which leads to a refocusing effect. Numerical simulations could provide a more detailed understanding of these effects, but would only yield meaningful results if the uncertainty of the actual, physical flip angles in the readout train is addressed.

The effects of the different spoiling methods on the in-vivo image quality are visible in a direct comparison. An interpretation of the locally reduced intensity in the image acquired with rewinder gradients as a susceptibility-related artifact from a gradient echo cannot be made with absolute certainty. Nevertheless, it is a plausible explanation, because the artifact is located at a tissue boundary with a sharp local change of the magnetic susceptibility.

The experiments on the effect of the two described modifications of the single-shot STEAM sequence show that these modifications improve its performance. The modified flip angle model and spoiling methods were therefore used in all MRI experiments with the single-shot STEAM sequence.

\subsection{Cardiac black-blood MRI using undersampled radial STEAM}

\subsubsection{Background}

Black-blood MRI is a an imaging technique where the signal from blood is suppressed. This can be beneficial for the image contrast in cardiac MRI, especially examinations of the myocardium. One pulse sequence for black-blood MRI is a double inversion recovery sequence [10]. This technique exploits T1 relaxation to selectively suppress the MR signal from spins flowing perpendicular to

the imaging plane using two $180^{\circ}$ pulses and a waiting time ("inversion time") in the order of T1 of blood before signal readout. The single-shot STEAM sequence has also been proposed for 
this black-blood MRI [15]. Besides avoiding susceptibility-induced artifacts, it does not require a long waiting time, thereby allowing better control of the heart cycle phase in which the image is acquired. The suppression of signal from moving spins is due to two physical effects. Firstly, a stimulated echo is only generated from protons which interact with both $90^{\circ}$ pulses as well as the low angle readout pulses, which are all slice-selective. This suppresses signal from blood flowing perpendicular to the imaging plane. Secondly, magnetic field gradients between the two initial $90^{\circ}$ pulses (time points $t_{1}$ and $t_{2}$ ), and between the low angle pulse (time point $t_{3}$ ) and the stimulated echo (time-point $t_{4}$ ), leads to a selective dephasing of moving spins. The phase of the precessing spins which move on a trajectory $\vec{x}(t)$ will be $\varphi=\gamma \int_{t_{1}}^{t_{2}} \vec{G}(t) \cdot \vec{x}(t) d t-\gamma \int_{t_{3}}^{t_{4}} \vec{G}(t) \cdot \vec{x}(t) d t$. Since the condition $\int_{t_{1}}^{t_{2}} \vec{G}(t) d t=\int_{t_{3}}^{t_{4}} \vec{G}(t) d t$ must be fulfilled so that the stimulated echo is not dephased, the phase for a stationary spin would be 0 , but a spin moving at velocity $\vec{v}$ would acquire the phase $\varphi=\gamma \vec{v} \cdot\left(\int_{t_{1}}^{t_{2}} \vec{G}(t) t d t-\int_{t_{3}}^{t_{4}} \vec{G}(t) t d t\right)$. This effect, primarily caused by the FID spoilers, dephases the spins in flowing blood. However, to a lesser extent, this also dephases the spins in moving parenchymal tissue, in particular a beating heart. Therefore, images cannot be acquired with good quality during systole, i.e. the "pumping" phase of the heart cycle with fast contraction of the myocardium, but only during diastole, i.e. the "resting phase" between the contractions, when there is relatively little motion. This is achieved by electrocardiographic (ECG) triggering. The ECG signal is measured in the patient using electrodes and transmitted to the control unit of the MRI device. Image acquisition is triggered at a defined time point after the systolic peak, which has to be experimentally adjusted to the heart cycle of each subject.

\subsubsection{Methods}

For this thesis, experiments on cardiac blood MRI using radial single-shot STEAM were limited to a proof of principle because the main focus of this work is on diffusion-weighted STEAM MRI. The single-shot STEAM sequence as described in section 3.1 was applied, preceded by a CHESS pulse to suppress signal from fat in the abdominal tissue. The ECG trigger delay was experimentally optimized for each volunteer, this was possible due to an available online implementation of the NLINV reconstruction algorithm for radial trajectories on a graphical processing unit (GPU). The orientation of the imaging plane is given by the short-axis view [17], which is an oblique image orientation allowing a view of the myocardium, and the right and left heart chambers. Images were acquired at an in-plane resolution of $2 \mathrm{~mm}$, a slice thickness of $6 \mathrm{~mm}$, a field of view of 256 $\mathrm{mm}$, and a sampling trajectory of 43 spokes acquired in a sequential clockwise order. Two image reconstruction methods were compared: NLINV and "gridding reconstruction", i.e. gridding and Fourier transform for the individual coil elements i, and calculation of the final image $D=\sqrt{\sum_{i}\left|d_{i}\right|^{2}}$ with $d_{i=1, \ldots, n}$ being the images reconstructed using the individual coil elements. 


\subsubsection{Results}

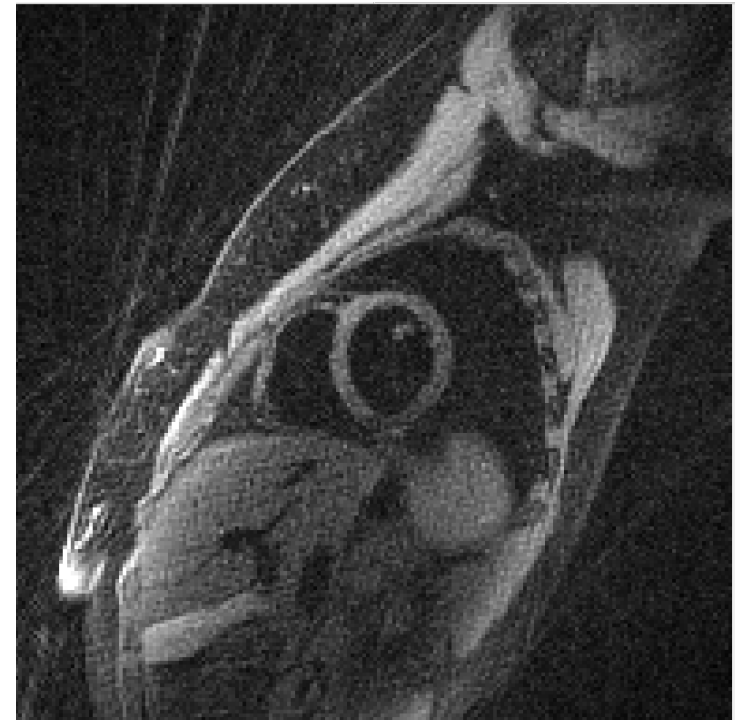

(a) Volunteer 1, gridding reconstruction

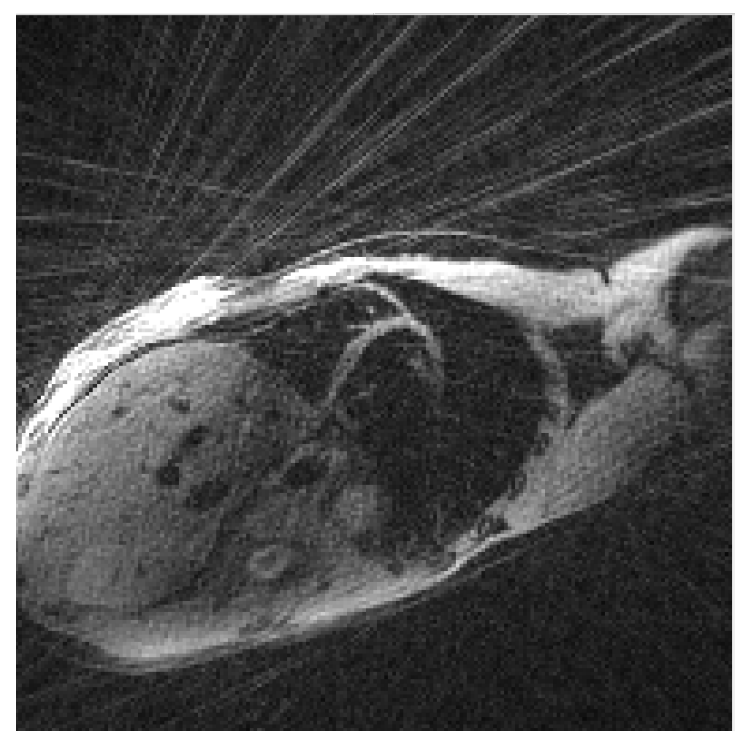

(c) Volunteer 2, gridding reconstruction

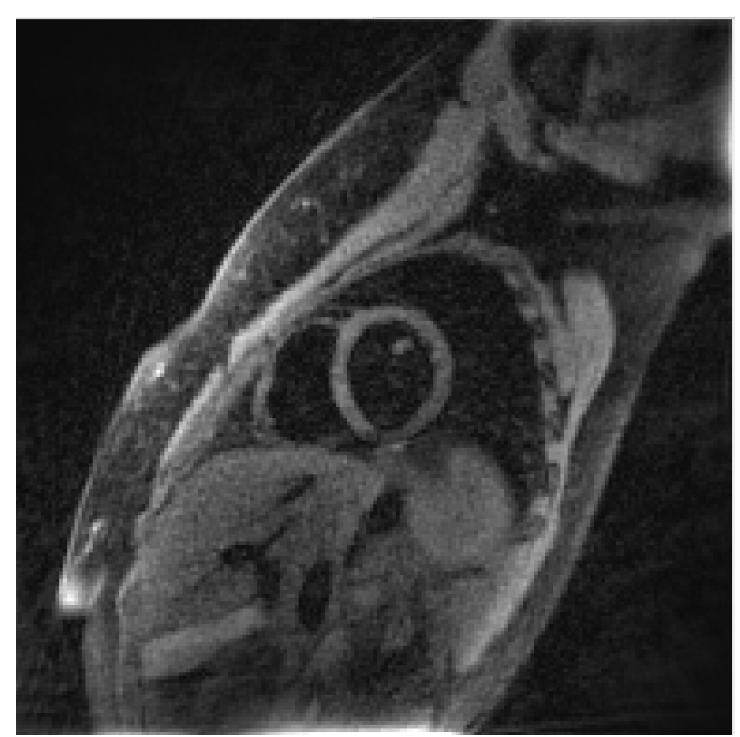

(b) Volunteer 1, NLINV reconstruction

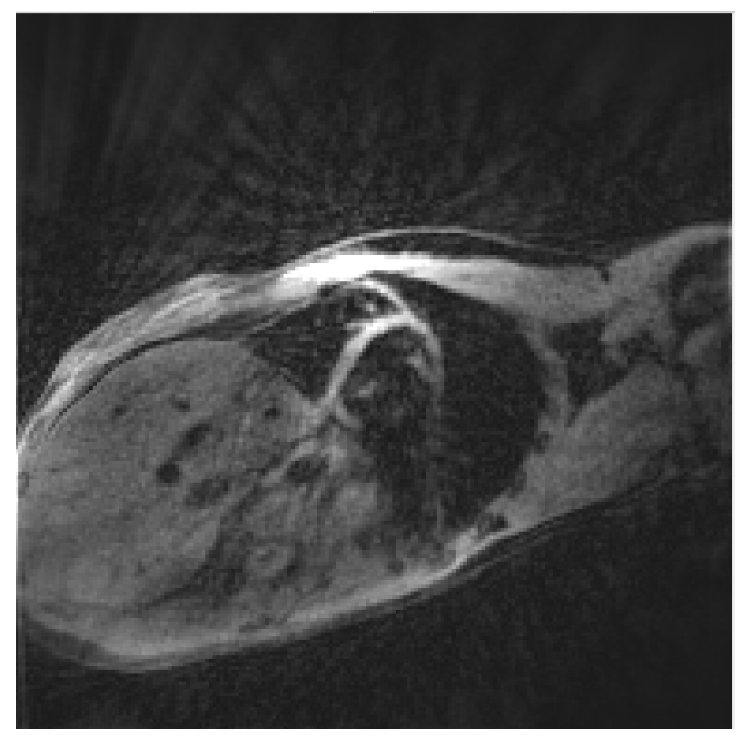

(d) Volunteer 2, NLINV reconstruction

Figure 16 - Cardiac black-blood images acquired with the single-shot STEAM sequence.

Figure 16 shows the resulting image quality for the two different volunteers, and the two reconstruction methods. NLINV reconstruction yields an image quality which is clearly superior to gridding reconstruction, with far less streaking artifacts caused by data undersampling. Moreover, a clear improvement of the signal-to-noise ratio is observed, which can be attributed to the suppression of noise due to the $L_{2}$ regularization, as well as the more efficient use of the sampled data from parallel imaging due to an estimation of the coil sensitivities.

The achievable quality of the cardiac images differs strongly between the two volunteers. The image acquired on volunteer 1 shows a good contrast between the the myocardial wall and the 
blood in the heart, due to a successful suppression of the MR signal from blood. The entire section of the myocardium delineating the two heart chambers is well visible without major artifacts. However, this image quality was not reproducible in most other volunteers. For volunteer 2, the image intensity is very low in a segment of the myocardium. This signal blackout is accompanied by streaking artifacts. The myocardial region which appears dark in the image is independent of the reconstruction method.

\subsubsection{Discussion}

The general image quality which was achieved for volunteer 1 demonstrates the successful development of the radial single-shot STEAM sequence and the potential of this sequence, in combination with NLINV reconstruction, for cardiac black-blood MRI. This is a new result, since the only previous attempt to apply the radial single-shot STEAM sequence to cardiac black-blood MRI [35] resulted in images corrupted by hyperintense representation of the abdominal and streaking artifacts for acquisition with 64 radial spokes.

Nevertheless, the results also show that good image quality and a complete representation of the myocardial tissue in the image section are not reproducible. This indicates that the dephasing effect due to the FID spoilers even compromises image acquisition during diastole. Dephasing of spins in cardiac tissue may be reduced by altering the strength and spatial direction of the FID spoiling gradients. In the case of weaker spoiling, this implies the risk of a corruption of the images by spurious gradient echoes generated by the radiofrequency pulses of the readout train.

An important factor for the choice of the optimal sampling trajectory for black-blood cardiac MRI is the dependence of the dephasing effect on the time point of signal readout. The phase of spins moving at a constant velocity is proportional to the time difference between the initial FID spoiler and the compensating gradient in the readout train. This means that the dephasing effect is strongest for the last acquired cartesian lines or radial spokes. For a cartesian sampling trajectory with a centric ordering of cartesian lines, where k-space is sampled from the center outwards, this leads to a greater sensitivity for the outer k-space lines to dephasing effects and an ensuing risk of local blurring for the myocardium in the phase encoding direction. For radial sampling trajectories, on the other hand, the signal losses in the radial spokes acquired late in the readout train lead to signal losses in the k-space center, as well as the observed streaking artifacts which can corrupt the entire image. A golden-angle-based sampling trajectory [69] where the angle between subsequent radial spokes is $137.5^{\circ}$ would make the pattern of possible signal losses more symmetric around the

k-space center. Nevertheless, the risk of streaking artifacts would persist. These considerations suggest that for cardiac black-blood STEAM MRI, a cartesian sampling trajectory, which has been applied successfully [34] may be the better choice, and it has the additional advantage of enabling image acquisition with a rectangular field of view.

The independence of the signal blackout observed in volunteer 2 from the reconstruction method 
proves that this signal loss is an artifact of image acquisition and not of image reconstruction. This, as well as the result from volunteer 1, shows that the nonlinear inversion algorithm is a suitable reconstruction algorithm for cardiac black-blood STEAM MRI. This finding contradicts the claim made in ref. 35] that coil sensitivity estimation as proposed by Uecker et al. 60, 62 needs to be further developed for the reconstruction of cardiac black-blood STEAM images from radially sampled data.

The following sections describe the use of the radial single-shot STEAM sequence for diffusionweighted MRI. 


\section{Diffusion-weighted STEAM MRI of the brain}

\subsection{Introduction}

The first use of the single-shot STEAM sequence for diffusion-weighted MRI was reported in 2000 by Nolte et al. [47]. This was achieved by replacing the excitation pulse of a cartesian single-shot STEAM sequence with a diffusion-weighted spin echo. This sequence allows diffusion-weighted imaging without susceptibility artifacts, because all k-space lines are acquired with radiofrequencyrefocused echoes, hence all susceptibility-induced phase differences between spins are refocused. This is the main advantage of this sequence over the EPI sequence described in section 2.1.3. At the same time, the diffusion-weighted (DW) STEAM sequence avoids the high RF energy absorption rates produced by spin echo sequences due to multiple refocusing RF pulses with high flip angles.

One important application of the DW single-shot STEAM sequence has been diffusion tensor imaging for nerve fiber tractography. The avoidance of susceptibility-induced image distortions has enabled anatomically correct localization and mapping of major nerve fiber bundles in the corpus callosum [26]. Moreover, specific fiber tractography applications which are very challenging with EPI due to adjacent boundaries of regions with highly different magnetic susceptibilities (air, soft tissue, bone) have been enabled by the diffusion-weighted STEAM sequence. One important example is tractography of the optic nerve [27].

The main factor which has limited widespread clinical applications of the DW single-shot STEAM sequence is the generally low signal-to-noise ratio compared to other diffusion-weighted imaging sequences, particularly EPI (e.g. [71]). This is firstly due to the fact that the maximal amplitude of a stimulated echo is half of the maximal amplitude of the spin echo. Secondly, replacing the excitation pulse by a diffusion-weighted spin echo with the echo time TSE leads to an additional T2-dependent signal loss, where the fraction of remaining signal is $e^{-\frac{T S E}{T 2}}$. Nevertheless, a recent comparative clinical study on the performance of EPI vs. DW single-shot STEAM in stroke diagnosis [36] showed that despite lower SNR, the STEAM sequence avoids diagnostic errors caused by susceptibility-induced image artifacts in practice. This promising result motivates a further development of diffusion-weighted STEAM imaging of the brain.

For this purpose of improving the signal-to-noise ratio and reducing the acquisition time, a novel image acquisition and reconstruction technique for diffusion-weighted STEAM MRI was developed based on undersampled radial k-space trajectories and nonlinear inverse image reconstruction. This section describes the details of this technique, the optimization of imaging parameters, as well as the results achieved in preliminary trials on image quality in healthy volunteers as well as patients.

A summary of the methods and results presented in this section has been published in a scientific paper [45]. 


\subsection{The diffusion-weighted undersampled radial single-shot STEAM se- quence}

\subsubsection{Sequence design}

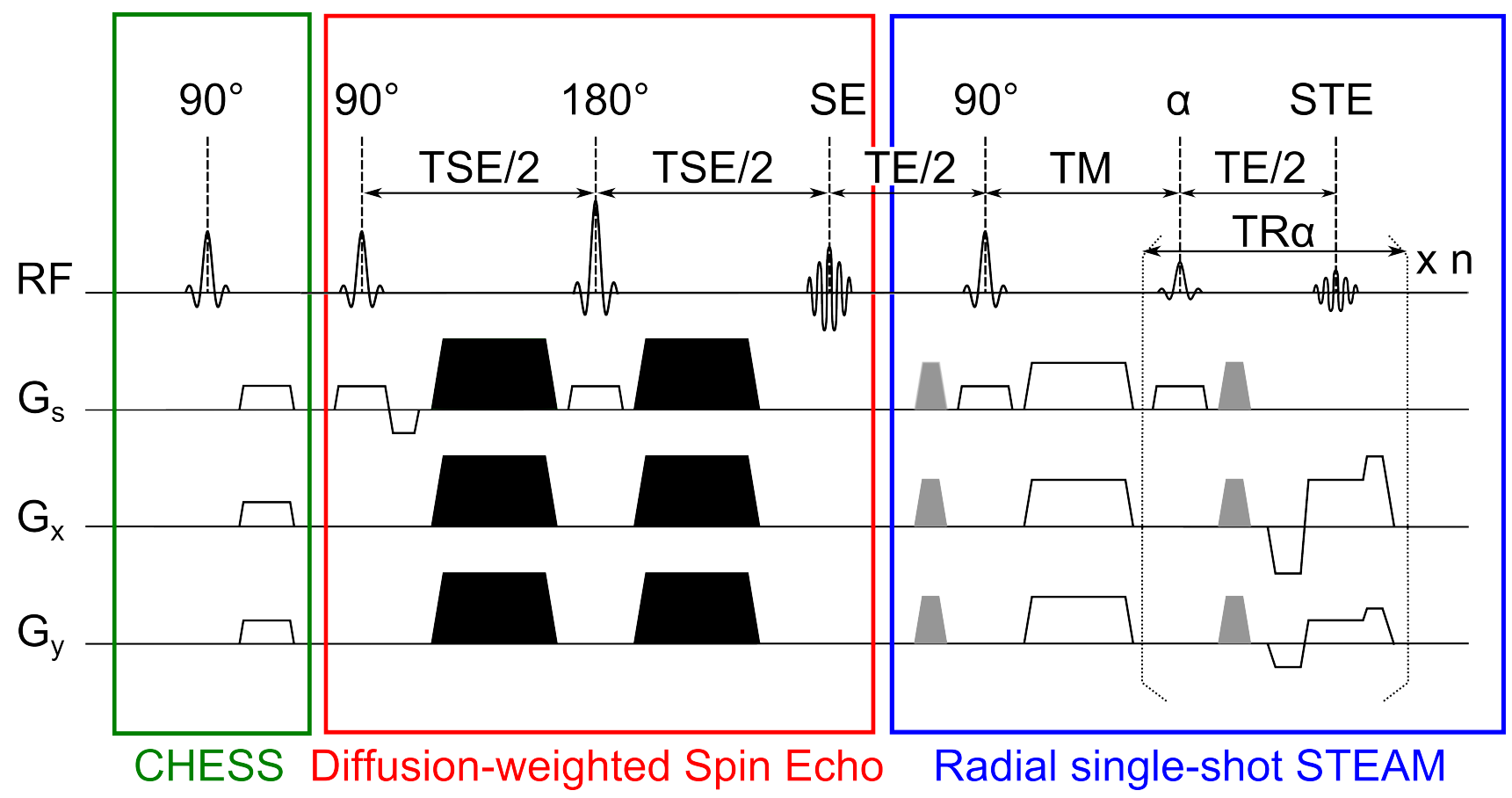

Figure 17 - The radial diffusion-weighted single-shot STEAM sequence

The extension of the radial single-shot STEAM sequence to a diffusion-weighted imaging sequence follows the same principle as for cartesian sampling [47]. In the STEAM sequence as described in section 3 , the initial $90^{\circ}$ excitation pulse is replaced by a diffusion-weighted spin echo. For the acquisition of the image with $b=0$, the diffusion gradients (black in the sequence diagram in figure 17) are replaced by spoiler gradients directly preceding and following the $180^{\circ}$ pulse with a negligible b-value. This prevents the occurrence of spurious radiofrequency echoes by dephasing unwanted transverse magnetization. This sequence is preceded by a CHESS pulse for the suppression of fat signal [20], see section 2.1, to suppress streaking artifacts from the fat tissue in the scalp. The radial diffusion-weighted STEAM sequence is shown in figure 17.

\subsubsection{Multi-slice imaging}

Diffusion-weighted multi-slice imaging of the entire brain requires the acquisition of multiple adjacent slices of tissue. While the slice profile of the DW-STEAM sequence can be described as the product of the slice profiles of the spin-echo, the second $90^{\circ}$ pulse, and the readout pulses, longitudinal magnetization is depleted in the much broader section of tissue which interacts with any of these components of the sequence. Hence, the subsequent acquisition of two adjacent slices will 
lead to considerable signal loss in the second slice. To avoid this effect, a time difference between the acquisition of any two neighboring slices is introduced: The sequence is first run subsequently on every second slice of the target volume, and then on the remaining slices. Pauses after each of these two sequence blocks are introduced to allow imaging with a predefined volume repetition time "TR". Acquisition of the target volume is first performed with $b=0$ (the "b0 image"), and repeated for each desired b-value and diffusion gradient direction. The b-values are generally given in units of $s \mathrm{~mm}^{-2}$, e.g. images acquired with $b=1000 \mathrm{~s} \mathrm{~mm}^{-2}$ are named "b1000". The orientation of the imaging slices is aligned with the anterior commissure $(\mathrm{AC})$ and the posterior commissure (PC), which is a very common choice in neuroimaging [53, 68]. Figure 18 shows the image orientation and the multi-slice acquisition scheme. To optimally take advantage of k-space coverage with any data regularization across multiple slices, the radial trajectory for each slice is rotated by an angle of $2 \pi z /(p * 5)$, with $p$ the number of radial spokes and $z$ an index which denotes the anatomical position of the slice. This rotation of the k-space trajectory has previously been used for the image frames of a time series in real-time MRI [63].

A naive implementation of this MRI sequence and direct application of nonlinear inverse reconstruction as described in section 2.3 yields images with several different types of artifacts. The development of a robust and artifact-free imaging technique required the experimental optimization of imaging parameters as well as further developments of the image reconstruction algorithm. These experiments and developments are described in the following two subsections.

\subsection{Optimization of image acquisition parameters}

Generally, the suppression of undesired physical effects leading to image artifacts involves a cost in imaging time and/or SNR. To achieve optimal image quality without unnecessarily high image acquisition times, the radiofrequency pulses, gradient pulses, and timing parameters of the diffusion-weighted STEAM sequence were tested in an extensive optimization process for brain imaging on a large number of volunteers. In this section, only a small and instructive subset of these experiments, as well as the effect of the respective imaging parameters on image artifacts is described:

1. The streaking artifacts which arise from fat in the scalp and their removal by a CHESS pulse are shown on a b0 image acquired with $300 \mathrm{~Hz} /$ Pixel.

2. The image artifacts which arise from spurious echoes in the acquisition of the b0 image and their removal by spoiling gradients before and after the $180^{\circ}$ pulse are demonstrated on STEAM images with $b=0$ of a phantom.

3. The emergence of susceptibility-induced distortions and false image intensities due to an FID spoiler of insufficient strength is demonstrated in vitro and in vivo on non-diffusion-weighted images. 


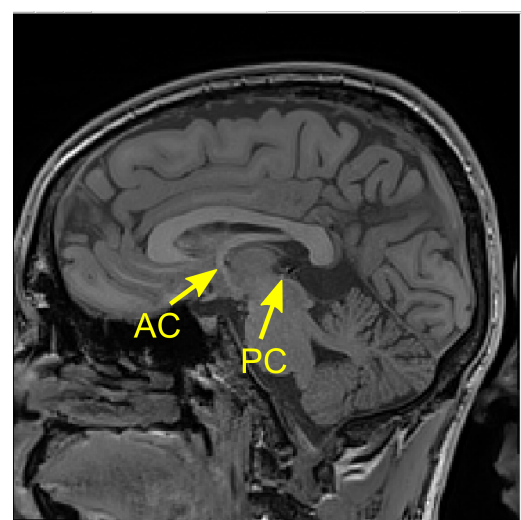

(a) Anterior commissure and posterior commissure

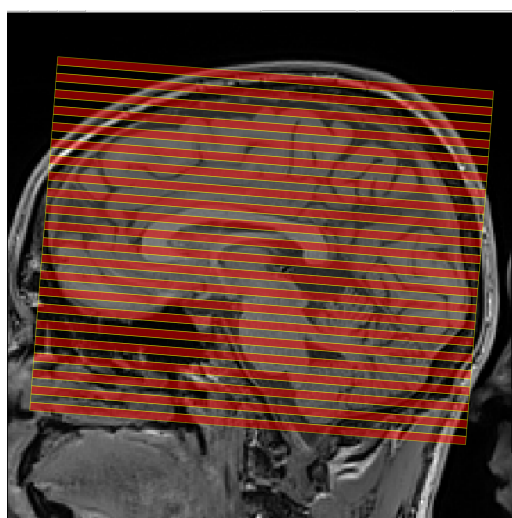

(b) Every second slice acquired in the first sequence block

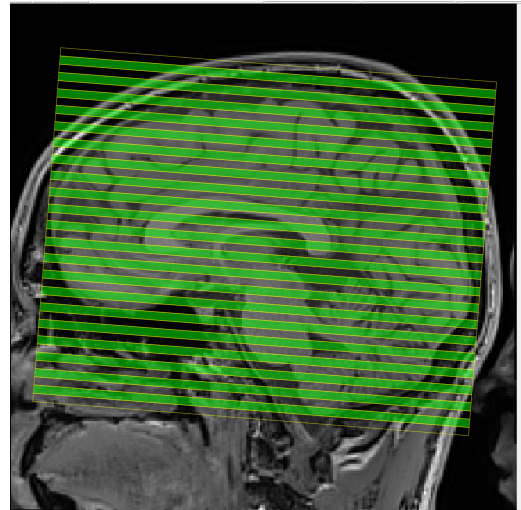

(c) Slices not acquired in the first sequence block

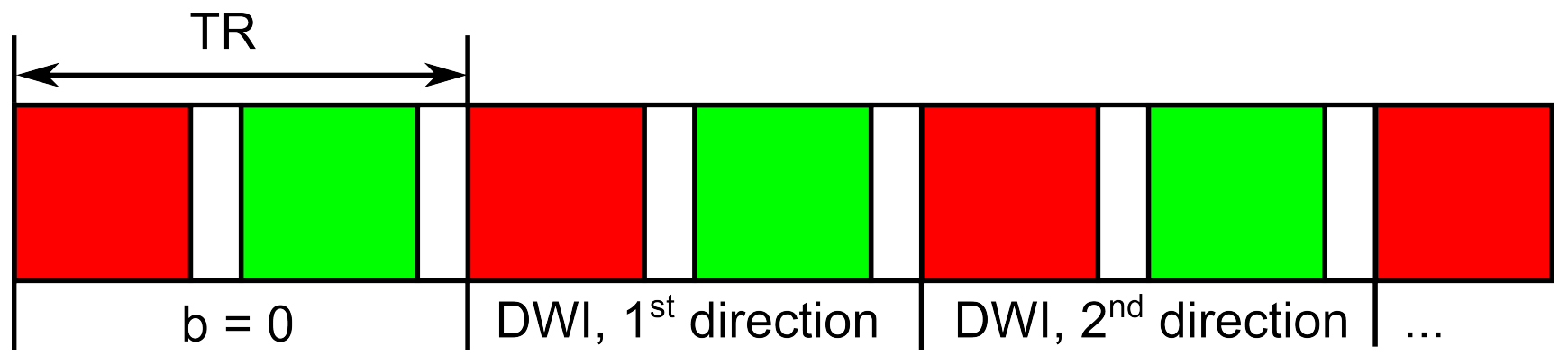

(d) Acquisition of every second slice (red), followed by the remaining slices (green) in two imaging blocks is repeated for all diffusion gradient directions, after acquisition of the b0 image.

Figure 18 - Diffusion-weighted imaging of the entire brain: Image orientation and multi-slice acquisition

4. The effect of the repetition time TR on image contrast is evaluated.

5. The removal of streaking artifacts in the diffusion-weighted image due to the new modification of the variable flip angle model is shown for the clinically relevant mean diffusion-weighted image with b1000.

\subsubsection{Fat suppression}

To asses the effect of fat suppression on the image quality, b0 images of the brain were acquired at $300 \mathrm{~Hz} /$ Pixel with and without a CHESS pulse for fat suppression. For brain imaging without fat suppression, the fatty tissue in the scalp produces the expected streaking artifacts, which are eliminated when the CHESS pulse is switched on (see figure 19). Although the streaking artifacts are less prominent for lower bandwidths, datasets acquired with $500 \mathrm{~Hz} / \mathrm{Pixel}$ are also visibly affected by the Larmor frequency shift of 420-440 Hz between water and fat. 


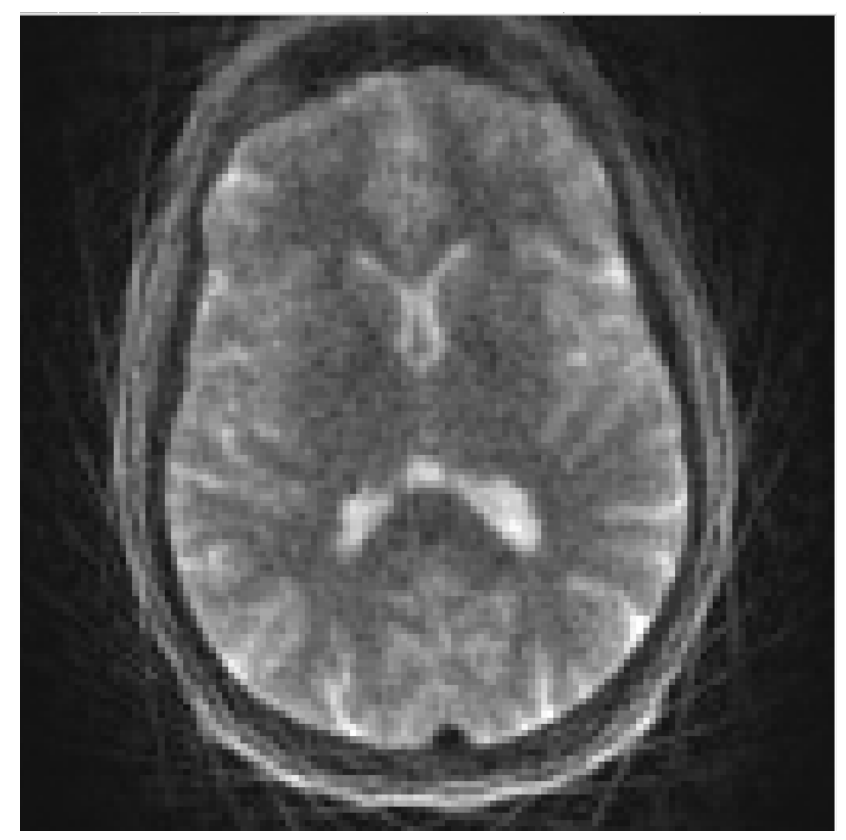

(a) Fat suppression off

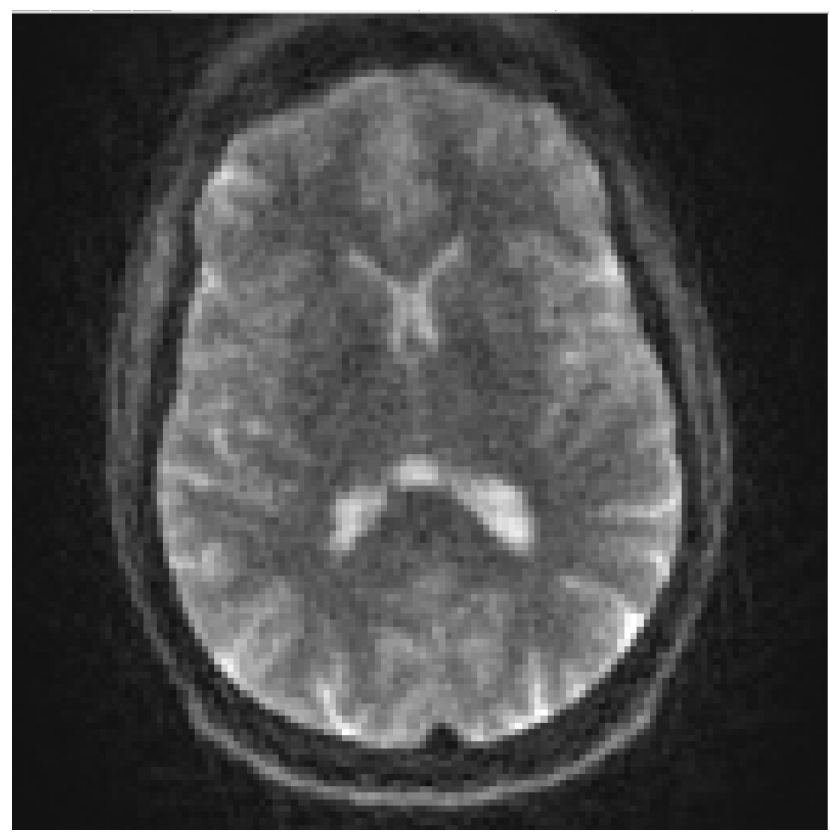

(b) Fat suppression on

Figure 19 - b0-image of the brain with and without fat suppression using a CHESS pulse

\subsubsection{Spoiling of the spin echo}

For the acquisition of the b0 image, the effect of the spoiling gradients on either side of the $180^{\circ}$ pulse of the spin echo can be seen from an imaging experiment on a phantom, with spoiler gradient amplitudes of 0,20 , and $40 \mathrm{mT} / \mathrm{m}$. As demonstrated in figure 20, a gradient amplitude of $40 \mathrm{mT} / \mathrm{m}$ sufficed to avoid visible artifacts from spurious RF echoes in the experiment shown here. For maximal robustness, a value of $50 \mathrm{mT} / \mathrm{m}$ was chosen for further experiments.

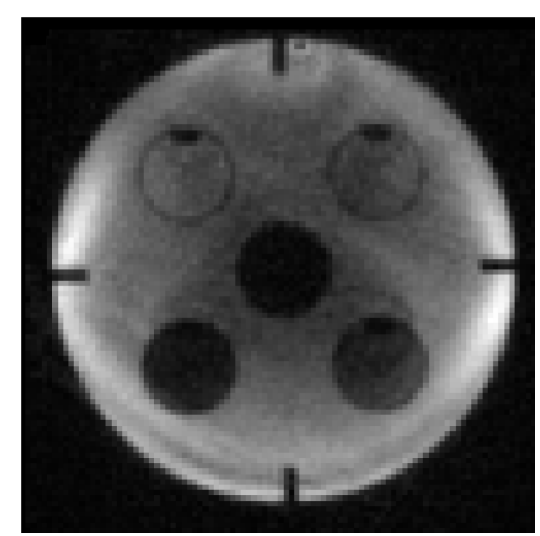

(a) No spoiling

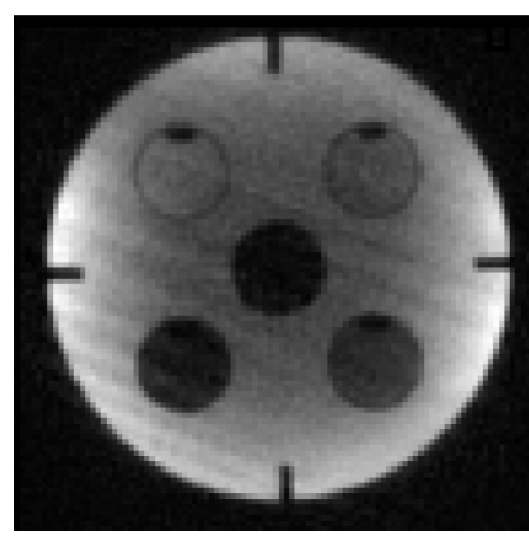

(b) $20 \mathrm{mT} / \mathrm{m}$

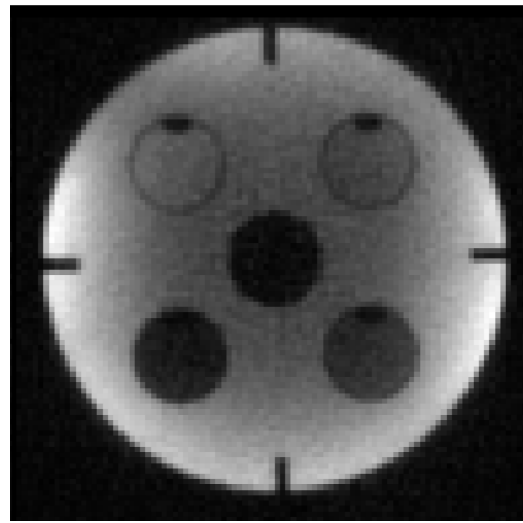

(c) $40 \mathrm{mT} / \mathrm{m}$

Figure 20 - Effect of spurious echoes in the diffusion module. The duration of the spoiler on either side of the spin echo was $3 \mathrm{~ms}$. 


\subsubsection{FID spoiling}

To investigate the artifacts related to insufficient FID spoiling, both a test tube and a slice of the human brain were imaged with the diffusion-weighted STEAM sequence with a b-value of 0 . The results are shown in figure 21. For image acquisition with a weak FID spoiler, both the image of the test tube and that of the brain slice exhibit a striped, periodic artifact pattern which indicates the superposition of two different oscillating signals. Furthermore, the air-water boundary in the test tube has a distorted shape as well as a hyperintensity in its vicinity. None of these artifacts are observed after an increase of the duration and amplitude of the FID spoiler.

Both the distortion and the false image intensities are typical susceptibility-induced artifacts caused by gradient echoes. This indicates that the stripe-like artifacts observed with a weak FID spoiler are caused by gradient echoes from the low-angle pulses of the readout train. The absence of all of these artifacts with a strong FID spoiler indicates effective dephasing of the FID signals in the readout train which suppresses gradient echoes.

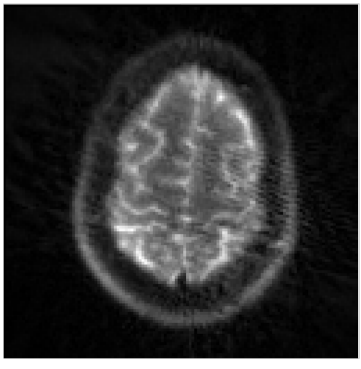

(a) Weak

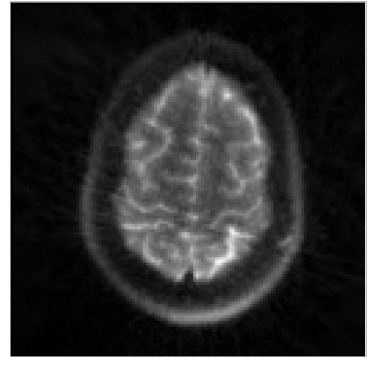

(b) Strong

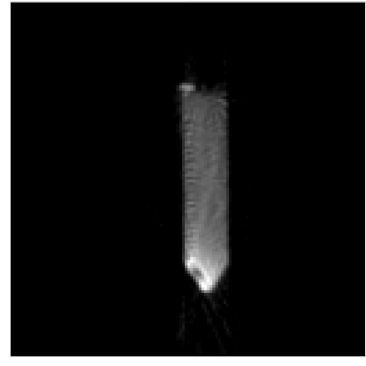

(c) Weak

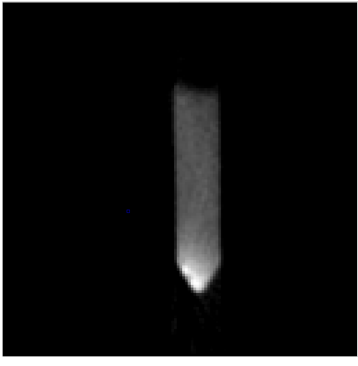

(d) Strong

Figure 21 - Suppression of gradient echo contributions by the FID spoiler. The two examined settings of the FID spoiler were "weak" (20 mT/m amplitude and a duration of $0.3 \mathrm{~ms})$ and "strong" (30 mT/m amplitude and a duration of $0.6 \mathrm{~ms}$ )

\subsubsection{Repetition time}

Figure 22 shows the effect of a repetition time of $8 \mathrm{~s}$ compared to longer repetition times on signal strength and contrast. Lowering the repetition time from $12 \mathrm{~s}$ to $8 \mathrm{~s}$ reduces the image intensity gray matter $(T 1=(1392 \pm 34) \mathrm{ms})$ more than in the white matter $(T 1=(838 \pm 18) \mathrm{ms})$ (relaxation times measured at 3T [67]). This can be seen in the reduced contrast between gray and white matter in the images. No visible differences in image contrast between repetition times of $12 \mathrm{~s}$ and $16 \mathrm{~s}$ were observed. This experiment shows that a repetition time of $12 \mathrm{~s}$ is optimal, and no longer repetition times are needed. 


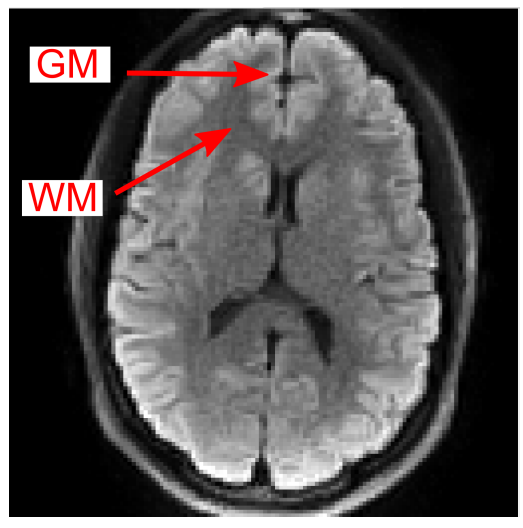

(a) $\mathrm{TR}=8 \mathrm{~s}$

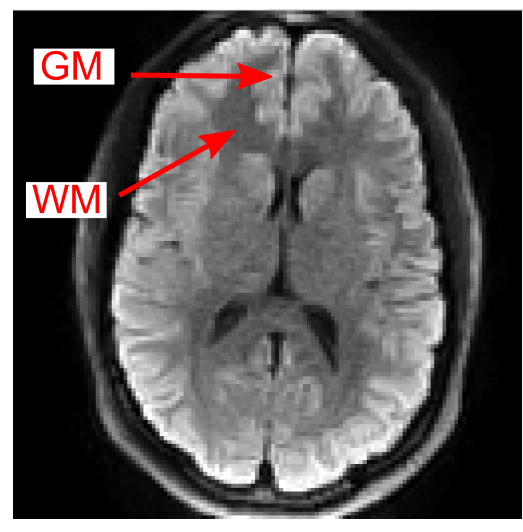

(b) $\mathrm{TR}=12 \mathrm{~s}$

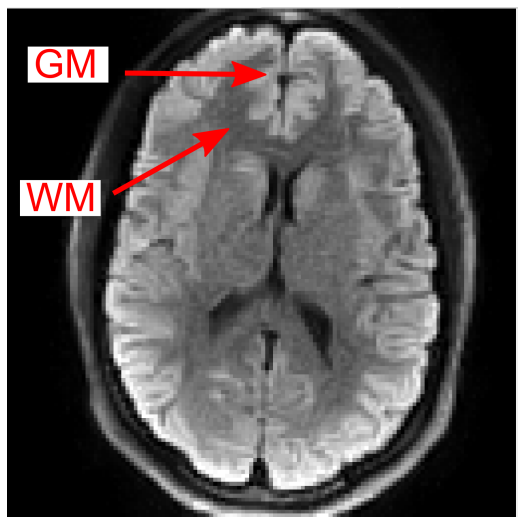

(c) $\mathrm{TR}=16 \mathrm{~s}$

Figure 22 - Dependence of image contrast on the repetition time. For a repetition time of $8 \mathrm{~s}$, a loss of image intensity in the gray matter (GM) is observed, which leads to a weaker contrast to white matter (WM).

\subsubsection{Maximal flip angle}

For diffusion-weighted brain imaging, the use of a maximal flip angle of $40^{\circ}$ avoids the local streaking artifacts in the mean diffusion-weighted image which occur with a maximal flip angle of $90^{\circ}$ in the variable flip angle scheme (see figure 23 . The hyperintense radial spoke can be identified in the Fourier transform of the image acquired using $90^{\circ}$. This corresponds to the last acquired spoke. While a maximal flip angle of $40^{\circ}$ avoids the streaking artifacts observed at $\alpha_{\max }=90^{\circ}$, no loss of image quality is seen and the signal strength as quantified by the total $L_{2}$ norm of the image is reduced by less than $3 \%$. This shows that the modified flip angle scheme as described in section 3.1 .3 suppresses artifacts with minimal signal loss in the diffusion-weighted images. 


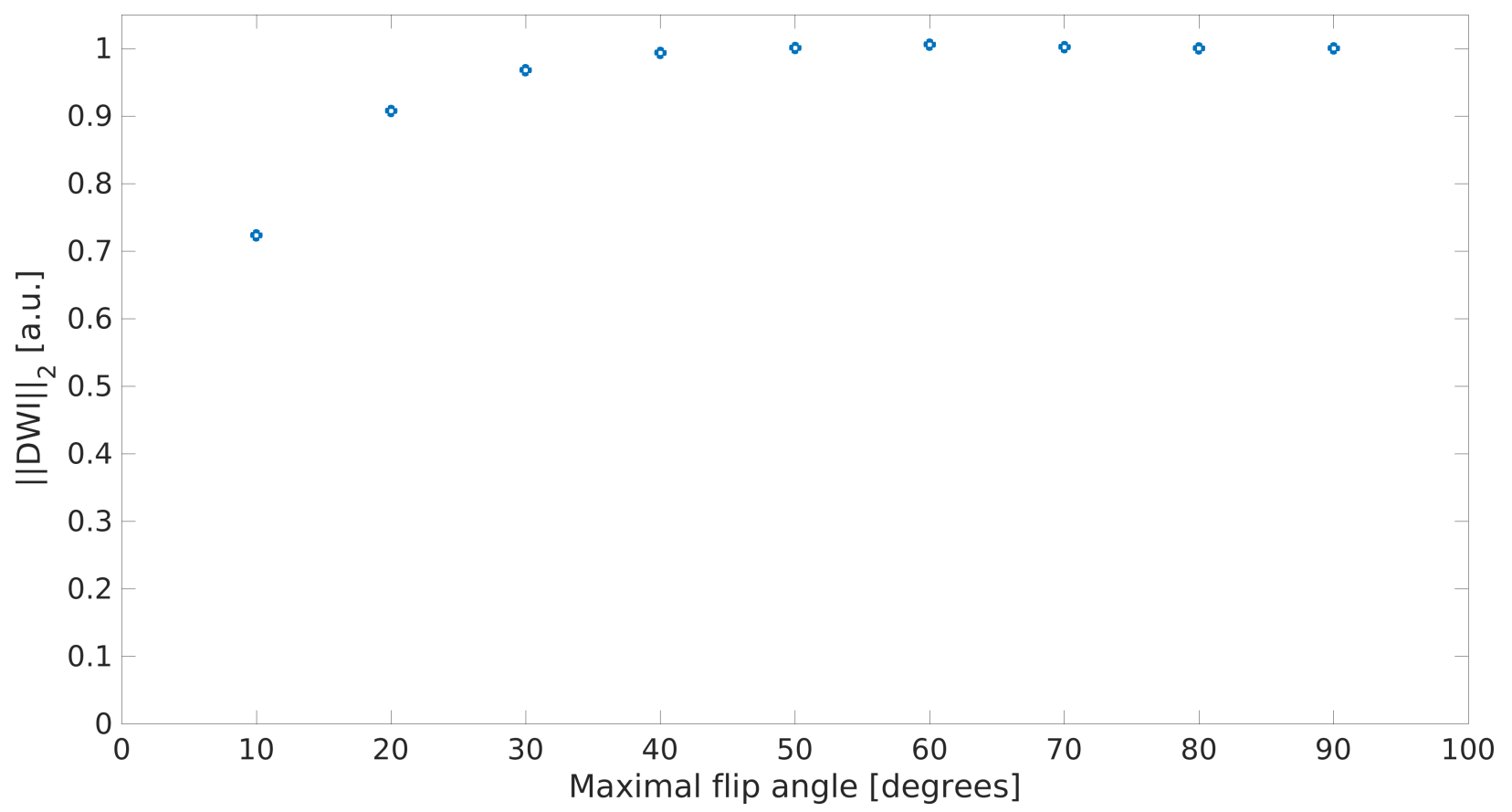

(a) Signal loss in the diffusion-weighted images quantified using the $L_{2}$ norm, as a function of the maximal flip angle.

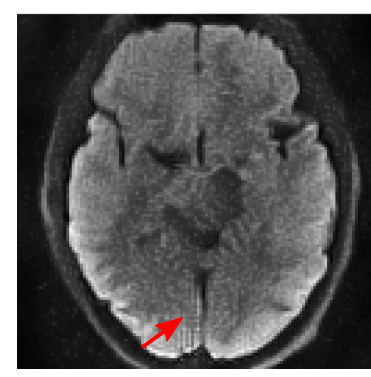

(b) $90^{\circ}$

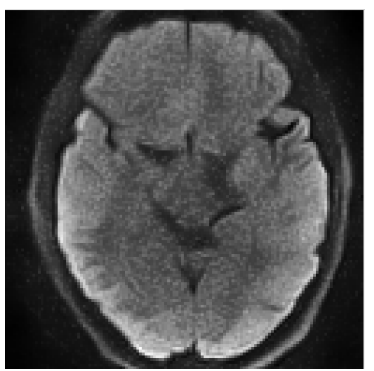

(c) $40^{\circ}$

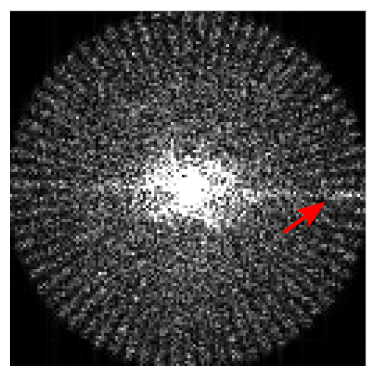

(d) $90^{\circ}$

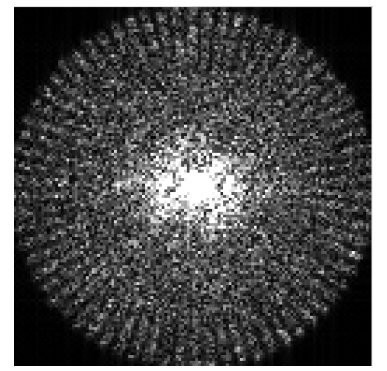

(e) $40^{\circ}$

Figure 23 - Effect of the maximum flip angle on image artifacts and signal loss. The images in the lower row show the mean diffusion-weighted image and its Fourier transform after image acquisition with $\alpha_{\max }=90^{\circ}$ and $\alpha_{\max }=40^{\circ}$. In the k-space images, the brightness is determined by the quantiles of the intensity distribution to visualize the radial spokes. The artifacts observed for $\alpha_{\max }=90^{\circ}$ are marked with red arrows. 


\subsection{Image reconstruction}

\subsubsection{Methods}

Overview For this work, the problem of reconstructing diffusion-weighted images from raw data of multiple slices in the brain acquired in parallel imaging was solved using the mathematical framework of nonlinear inversion described in section 2.3. To achieve robust image reconstruction, several developments were necessary:

- To allow for an accurate calculation of the diffusion tensor, non-DW images and DW images had to be reconstructed with a common set of coil sensitivities.

- This requires the same definition of virtual channels across multiple slices. Hence, the principal-components-based data compression technique required adaptation to a variable number of slices.

- An additional regularization term was included in the reconstruction algorithm to exploit the similarity of the coil sensitivities of neighboring slices for more stable reconstruction results.

- To achieve a stable and well-defined degree of image regularization, a new method for data scaling and initialization based on the properties of the acquired data was developed.

This section describes the entire data processing pipeline including these developments. The final results are the b0 image, the mean diffusion-weighted image, and the ADC map. An overview of this pipeline is given in figure 24 .

The developments for preprocessing, image reconstruction, and post-processing are described in the following paragraphs.

Preprocessing In real-time MRI with nonlinear inverse reconstruction [62, 63], the amount of data to be processed is reduced using a principal components analysis (PCA) of the raw data with respect to the receive channels and the selection of a predefined number of principal components for further processing. For DW STEAM imaging of the brain, the possibility of data regularization across the slices requires simultaneous reconstruction of multi-slice data with a variable number of slices. Since the number of principal components which is needed to adequately describe the data without introducing qualitative changes in the image content depends on the number of slices as well as the anatomical region, this number $k$ of principal components must be calculated from the properties of the raw data. Final image reconstruction requires a good image quality for each slice, therefore, the reduced dataset must provide a suitable description of every single slice. The criterion for the choice of $k$ is that the retained fraction of data variance in each slice shall be greater than or equal to a predefined value $f$. The calculation of $k$ is derived as follows:

For a total number $\mathrm{n}$ of receive channels, the principal component transform of a multi-slice set of raw data yields the normalized n-dimensional eigenvectors $\overrightarrow{v_{1}}, \overrightarrow{v_{2}}, \ldots, \overrightarrow{v_{n}}$ and real and positive 


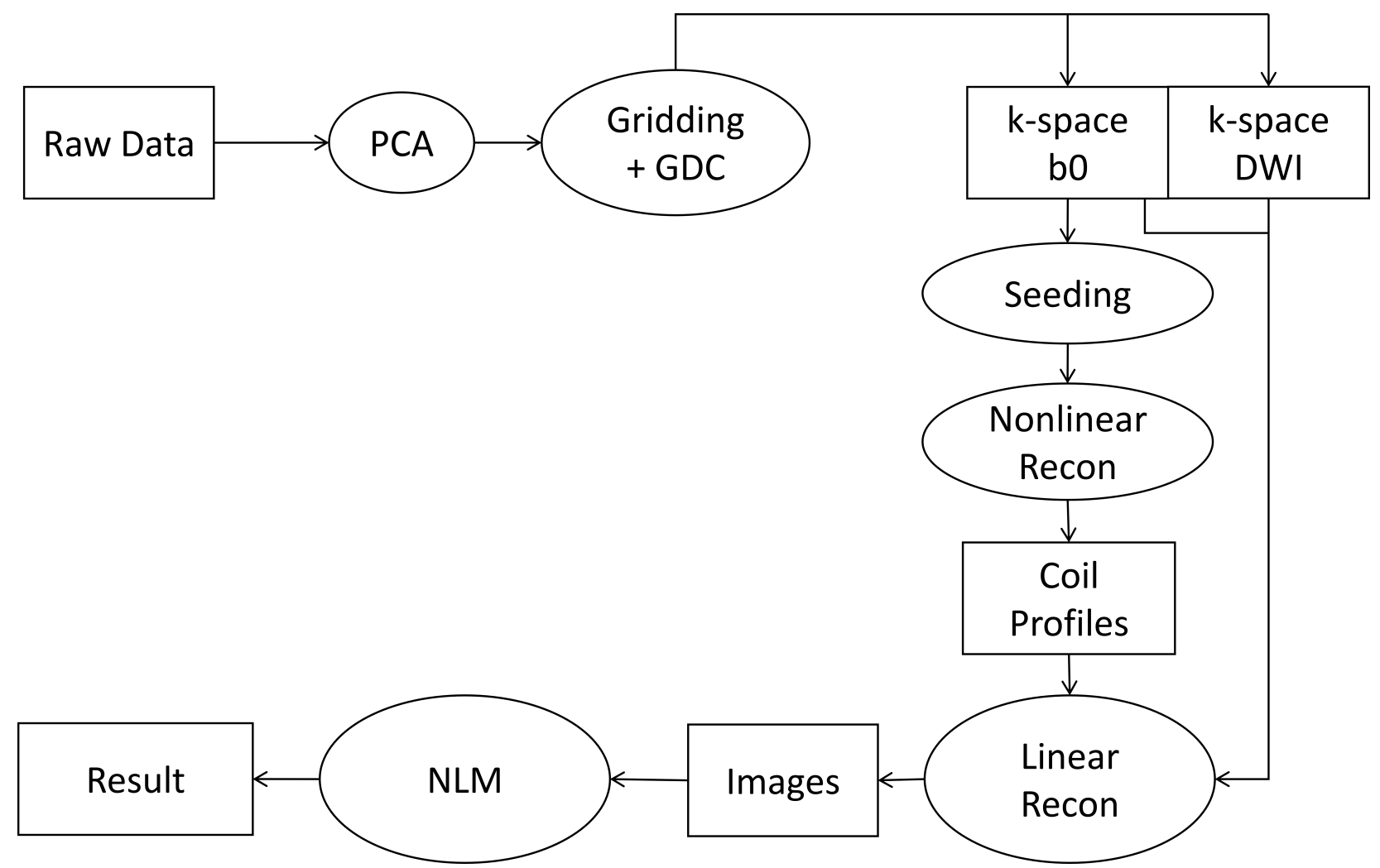

Figure 24 - The data processing pipeline for image reconstruction. The preprocessing steps, PCA-based channel compression, gridding, and a gradient delay correction (GDC) result in a cartesian representation of the acquired data in $\mathrm{k}$-space with a reduced number of virtual imaging channels. The b0 data are used to estimate the coil sensitivities (or coil profiles) by nonlinear reconstruction. These coil profiles are used for linear reconstruction of the image content of both the b0 image and the diffusion-weighted images. Post-processing involves denoising with a non-local means filter (NLM) and diffusion tensor calculation. The final results are the denoised b0 image, the mean diffusion-weighted image, and the ADC map.

eigenvalues $w_{1}, w_{2}, \ldots, w_{n}$ of the $n \times n$ covariance matrix of a multi-slice dataset, with $w_{1}>w_{2}>$ $\ldots>w_{n}$. The fraction of variance in the entire dataset which is retained on selection of the first $k<n$ principal components is $\frac{w_{1}+w_{2}+\ldots+w_{k}}{w_{1}+w_{2}+\ldots+w_{n}}$. For one slice, the raw data $D_{s}$ of slice $s$ can be represented as a $p \times n$ matrix, with a number of $p$ sampled data points. Data compression with $k$ principal components calculated on the multi-slice dataset can then be described as multiplication with the compression matrix $V=\left[\overrightarrow{v_{1}}, \overrightarrow{v_{2}}, \ldots, \overrightarrow{v_{k}}\right]$, the compressed data $C_{s}=D_{s} V$. The fraction of retained variance then becomes $g_{s}(k)=\frac{\sum_{i=1}^{k} \operatorname{Var}\left(c_{s, i}\right)}{\sum_{j=1}^{n} \operatorname{Var}\left(d_{s, i}\right)}$ with $c_{s, i}$ and $d_{s, i}$ the i-th column vectors of $C_{s}$ and $D_{s}$. The selection rule for the number $k$ of virtual channels in a dataset of $S$ slices is therefore $k=\min \left\{m \in \mathbb{N} \mid g_{s}(m) \geq f \forall s \in \mathbb{N}, s \leq S\right\}$. The parameter $f$ was set to 0.95 .

Following PCA-based data compression, a gradient delay correction is applied to the raw data to account for deviations from the desired k-space trajectory caused by hardware imperfections [64, 48]. Then, the data are interpolated onto a cartesian grid using Kaiser-Bessel functions. 


\section{Reconstruction}

Coil sensitivity estimation with slice regularization The first step of the image reconstruction algorithm consists in an estimation of the coil sensitivities from the b0 data using nonlinear inversion. In a generalized form, the cost function to minimize can be described as

$$
K(\hat{x})=\|G(\hat{x})-Y\|_{2}^{2}+\frac{\beta}{2} R(\hat{x})
$$

with $\hat{x}=(\rho, \hat{c})=W^{-1}(x)$, the raw data $Y$ and the forward operator $G$ as defined in section 2.3 . and a generic real-valued regularization function $R$. If $R$ is quadratic in $\hat{x}$, the problem can be solved with the iteratively regularized Gauss-Newton method. In each Newton iteration $m$, the linearized problem to solve is

$$
\left(D G^{H} D G\left(\hat{x}_{m}\right)+\beta_{m} D^{2} R\left(\hat{x}_{m}\right)\right) d \hat{x}=-\beta_{m} D R\left(\hat{x}_{m}\right)+D G^{H}\left(\hat{x}_{m}\right)\left(Y-G\left(\hat{x}_{m}\right)\right)
$$

with the Fréchet derivative $D$. The problem is solved in a number $\mathrm{M}$ of Newton iterations, with $\beta_{m}=0.5^{m-1}$. The final regularization parameter is thus determined by the number of Newton iterations. With $R(\hat{x})=\|\hat{x}\|_{2}^{2}$, this algorithm is equivalent to NLINV in its original form. To take advantage of the similarity of the coil sensitivities of neighboring slices to achieve a more stable estimation of the sensitivities, the regularization term is extended to

$$
R(\hat{x})=\|\hat{x}\|_{2}^{2}+d \sum_{i=1}^{k} \sum_{s=1}^{S-1}\left\|\hat{c}_{i, s}-\hat{c}_{i, s+1}\right\|_{2}^{2}
$$

where $\hat{c}_{i, s}$ is the transformed coil sensitivity of the $\mathrm{i}$-th virtual channel and the s-th slice, $\mathrm{k}$ the number of virtual channels and $\mathrm{S}$ the number of slices. The slice regularization strength d determines the desired degree of similarity of the coil sensitivities of neighboring slices. Differentiation of R w.r.t. $\hat{x}$ yields $D R=2(\rho, L \hat{c})$ and $D^{2} R d \hat{x}=2(d \rho, L d \hat{c})$, with

$$
L=I+d\left(\begin{array}{ccccc}
1 & -1 & 0 & 0 & \ldots \\
-1 & 2 & -1 & 0 & \ldots \\
\ldots & \ldots & \ldots & \ldots & \ldots \\
\ldots & 0 & -1 & 2 & -1 \\
\ldots & 0 & 0 & -1 & 1
\end{array}\right), \hat{c}=\left(\begin{array}{c}
\hat{c}_{s=1} \\
\ldots \\
\ldots \\
\ldots \\
\hat{c}_{s=S}
\end{array}\right)
$$

and the identity matrix $I$, i.e., the matrix L acts on the coil sensitivities represented as a column vector of slices. As in the original NLINV algorithm, the linearized problem for each iteration is solved using the conjugate gradient algorithm.

Linear reconstruction With known coil sensitivities, image reconstruction becomes a linear problem. Mathematically, this can be formulated as the NLINV reconstruction problem, with the 
coil sensitivities as constants. The forward operator reduces to:

$$
\tilde{F}(\rho)=P_{k} \mathcal{F} P_{F O V} C \rho
$$

where $C$ denotes multiplication with the coil sensitivities,

$$
C: \rho \mapsto\left(\begin{array}{c}
c_{1} \rho \\
\cdots \\
c_{k} \rho
\end{array}\right)
$$

The derivative and its adjoint with respect to $\rho$ are

$$
D \tilde{F}(\rho) d \rho=\tilde{F}(d \rho), D \tilde{F}^{H}(\rho) Y=\tilde{F}^{H}(Y)=\sum_{i=1}^{k} c_{i}^{*} P_{F O V} \mathcal{F}^{-1} P_{k} Y .
$$

The reconstruction problem with an $L_{2}$ regularization on $\rho$ can be represented as the minimization of the cost functional:

$$
K(\rho)=\|\tilde{F}(\rho)-Y\|_{2}^{2}+\frac{\beta}{2}\|\rho\|_{2}^{2}
$$

with the same parameter $\beta$ as in equation 11 . The cost functional $K$ is minimized by computing

$$
\rho=\left(\tilde{F}^{H} \tilde{F}-I \beta\right)^{-1} \tilde{F}^{H} Y
$$

using the conjugate gradients method. In this reconstruction, the scaling of the input coil sensitivities influences the degree of image regularization: multiplication of the sensitivities by a factor $a$ alters the balance between the terms $\tilde{F}^{H} \tilde{F}$ and $I \beta$ in equation 18 and has the same effect on the final image as multiplying the regularization parameter $\beta$ by $a^{-2}$, i.e., strengthening or weakening the influence of the regularization term of the cost function. Optimal regularization must balance image blurring from high values of $\beta$ against image noise resulting from low values of $\beta$. Therefore, well-defined scaling properties are required for the output of the nonlinear reconstruction which estimates the coil sensitivities.

Seeding For the nonlinear reconstruction, the data consistency term of the cost function $\mathrm{K}$ in equation 11 is invariant to a rescaling of the form $(\rho, \hat{c}) \rightarrow\left(a \rho, a^{-1} \hat{c}\right)$ with a real positive number $a$. Hence, the scaling of the estimated coil sensitivities is determined only by the regularization term, which favors $\|\rho\|_{2}=\|\hat{c}\|_{2}$. To guarantee predictable results of the linear reconstruction algorithm, this scaling must converge in the nonlinear reconstruction. Fast convergence motivates initialization (seeding) of the non-linear reconstruction algorithm with data arrays $\rho_{\text {init }}$ and $\hat{c}_{\text {init }}$ with $L_{2}$ norms which are similar to those predicted for the final result. Besides equal norms for the image and the coil sensitivities, data consistency requires $\|G(\hat{x})\|_{2}=\|Y\|_{2}$, which can be 
approximated by the condition $\left\|Y_{i, s}^{\prime}\right\|_{2}=\left\|P_{s} \mathcal{F}\left(\rho_{\text {init }, s} \circ c_{\text {init }, i, s}\right)\right\|_{2}$, where "o" denotes point-wise multiplication, $Y_{i, s}^{\prime}$ represent the raw data of the $\mathrm{i}$-th virtual channel and the s-th slice interpolated on a cartesian grid and $P_{s}$ is the Fourier transform of the point-spread function which is defined by the k-space trajectory used for the slice s. Besides defining the initial scaling of coil sensitivities and image content, seeding provides a first estimate of the intensity distribution in the image and the phase maps of the coil sensitivities. The image content is initialized as a real-valued array $\rho_{\text {init }, s} \sim \sqrt{\sum_{i=1}^{n}\left|\mathcal{F}\left(M \circ Y_{i, s}^{\prime}\right)\right|^{2}}$, where $M$ is a Gaussian smoothing mask, and the coil sensitivities are initialized as $\hat{c}_{i n i t, i, s} \sim\left(1+r\|\vec{k}\|^{2}\right)^{-\frac{l}{2}} Y_{i, s}$, with the parameters $\mathrm{r}$ and 1 equal to those which define in-plane coil sensitivity regularization in NLINV, and the arrays are normalized to fit the conditions for the $L_{2}$ norms described above.

Post-processing The reconstruction algorithm yields one b0 image and one diffusion-weighted image for each gradient direction. Post-processing of these "raw images" involves the use of a non-local means filter (NLM) [38] for image noise removal, which retains high spatial frequencies by averaging intensities of pixels with similar local environments. The following computation steps are taken to compute the final results, i.e. the b0 image, the mean diffusion-weighted image, and the ADC map

1. For protocols with multiple averages, the magnitudes of the reconstructed images are averaged.

2. The b0 image is computed by denoising the raw b0 image with NLM.

3. The mean diffusion-weighted image is computed by first averaging over the magnitudes of the raw DW images corresponding to the different gradient directions, then denoising with NLM.

4. For calculation of the ADC map, the following steps are performed

(a) Denoising of the raw images with NLM.

(b) Calculation of the diffusion tensor. For 6 gradient directions, an exact solution exists, for more gradient directions, an optimal solution is computed with a weighted linear least squares algorithm [37.

(c) The ADC is calculated as the mean eigenvalue of the diffusion tensor.

\subsubsection{Numerical experiments}

Channel compression In PCA-based channel compression, the number of image slices reconstructed at once clearly affects the number of principal components needed to correctly reconstruct 
the image content. As shown in figure 25, with the PCA calculated on one section, 5 principal components are sufficient to correctly reconstruct the image content. The retained fraction of variance, or energy, in the raw data was $93.3 \%$. With a PCA computed on 25 slices, the use of 5 principal components yields reconstructed images with visible local intensity changes; the retained fraction of energy was $76.6 \%$. With a joint reconstruction on 25 slices, 20 principal components (energy fraction of $97 \%$ ) yield a reconstructed image with good qualitative agreement with the image reconstructed without PCA. This shows that the retained energy fraction is a better predictor for the effect of data compression on image quality than the number of principal components, and that a minimal retained energy of $95 \%$ was a suitable choice.

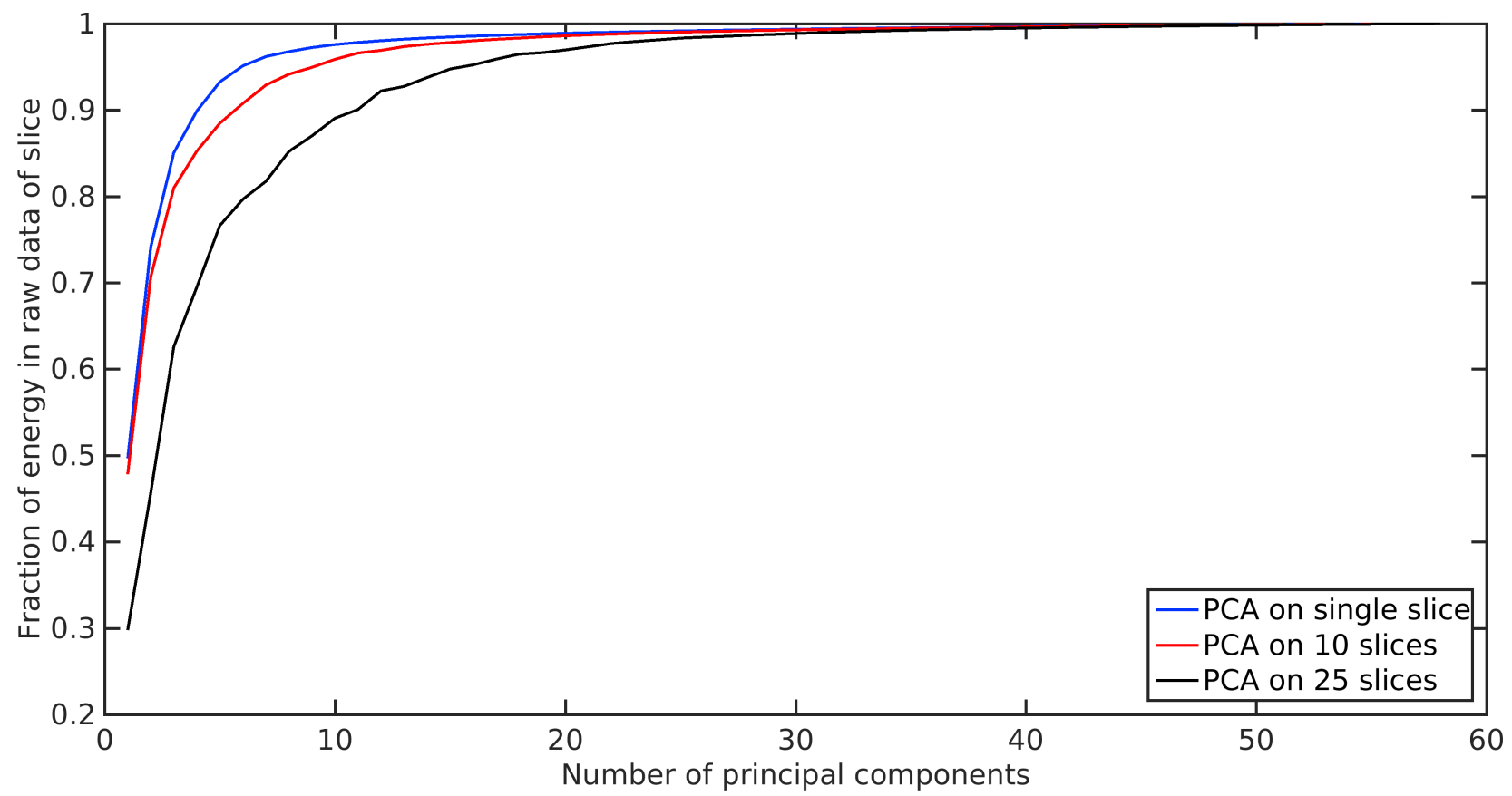

(a) Signal loss in the diffusion-weighted images as quantified by the fraction of retained energy in the raw data of a single slice as a function of the number of principal components

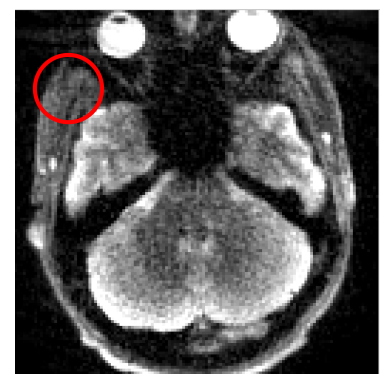

(b) No PCA

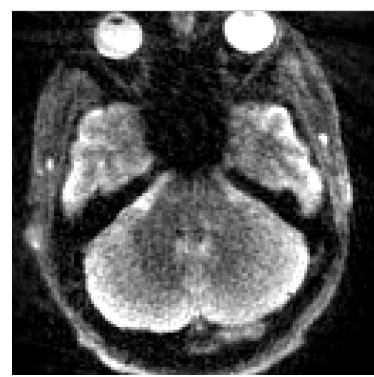

(c) 5 PC on 1 slice

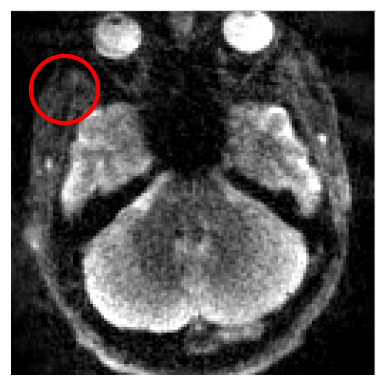

(d) 5 PC on 25 slices

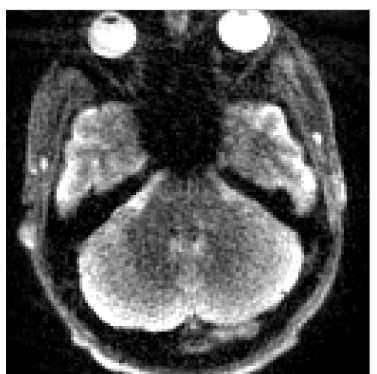

(e) $20 \mathrm{PC}$ on 25 slices

Figure 25 - Effect of number of principal components and the number of simultaneously reconstructed slices on the retained fraction of energy in the raw data. For too few principal components, alterations of image intensities are observed, e.g. in the region marked with a red circle. 
Data scaling and seeding To test the data scaling of the reconstruction algorithm with and without the proposed seeding method, a nonlinear inverse reconstruction with 8 Newton steps was run on the b0-data of a diffusion-weighted dataset of the brain multiple times, with the result $(\rho, \hat{c})$ from each run used as the initialization for the next run. As seen in figure 26, after trivial initialization of the estimates of image content (homogeneous value of 0 ) and coil sensitivities (homogeneous value of 1 ), the total $L_{2}$ norms of the image content and the coil sensitivities $\left(\|\rho\|_{2}\right.$ and $\|\hat{c}\|_{2}$ ) strongly vary for the first five runs, and remain nearly constant after the fifth run. The scaling to which $\|\rho\|_{2}$ and $\|\hat{c}\|_{2}$ tend after multiple runs of the reconstruction algorithm is already achieved after one reconstruction run when the proposed seeding method is used.

The effect of data scaling on the image quality can be seen in figure 27. After coil sensitivity estimation with one run of the reconstruction algorithm, subsequent linear image reconstruction yields a strongly blurred image. This strongly differs from the result of the nonlinear reconstruction. The blurring indicates excessively strong image regularization in the linear reconstruction step, which is consistent with the low norm of the coil sensitivities resulting in a downscaling of the data consistency term relative to the regularization term for the linear reconstruction.

The use of coil sensitivities estimated with ten runs of the nonlinear reconstruction or one run with the proposed seeding method yields linearly reconstructed images which are consistent with the nonlinear reconstruction result. In these cases, the scaling of the coil sensitivities had converged in the nonlinear reconstruction.

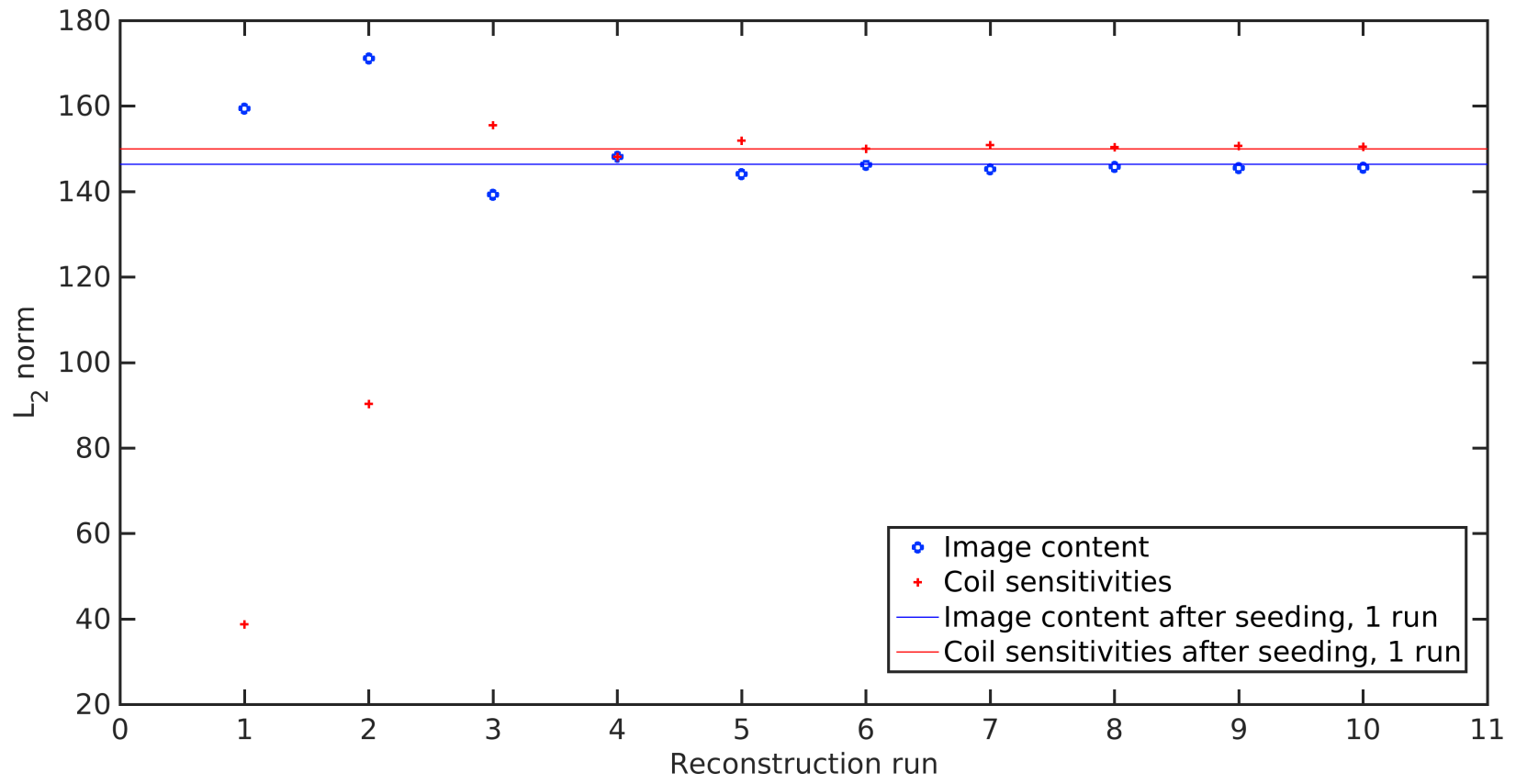

Figure $26-L_{2}$ norm of the image content $\rho$ and the transformed coil sensitivities $\hat{c}$ after nonlinear reconstruction for repetitive runs of the reconstruction algorithm with 8 Newton steps and after seeding. 


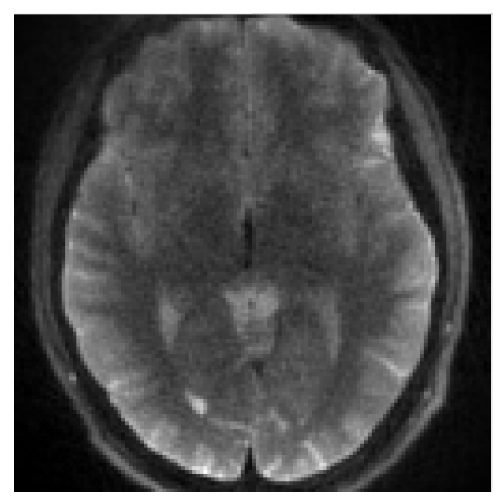

(a) Nonlinear reconstruction, no seeding, image after 1 run

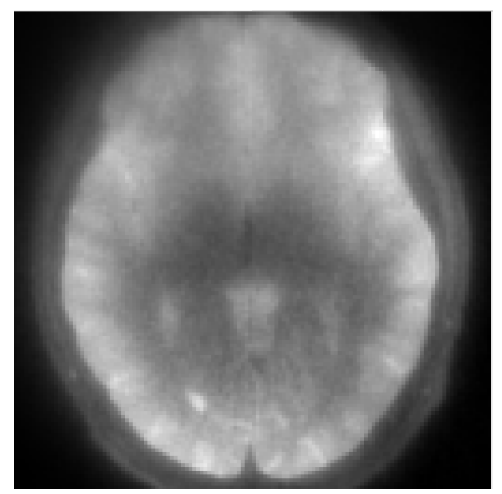

(d) Linear reconstruction, no seeding, image after 1 run

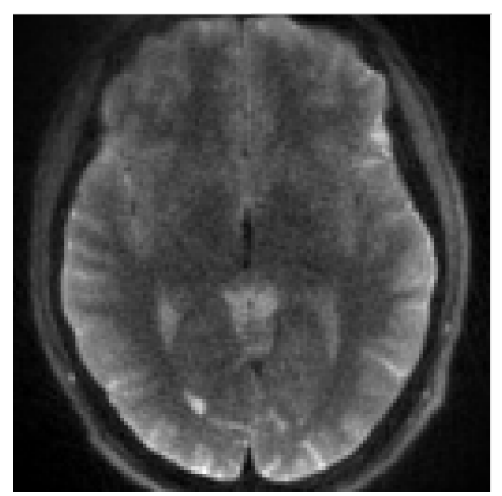

(b) Nonlinear reconstruction, no seeding, image after 10 runs

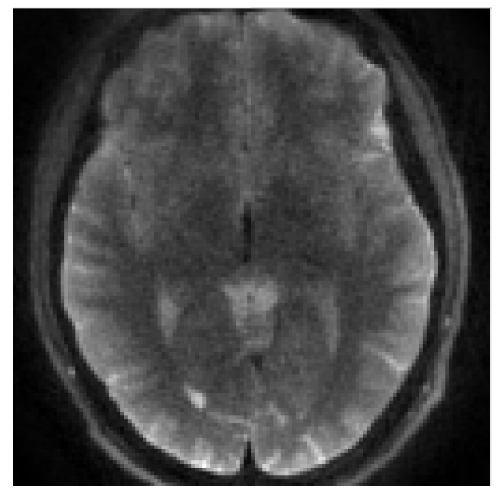

(e) Linear reconstruction, no seeding, image after 10 runs

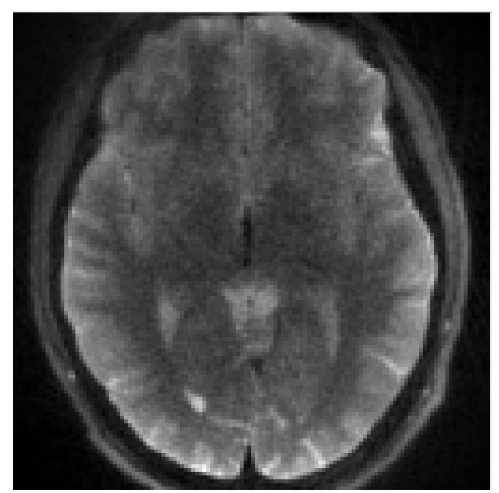

(c) Nonlinear reconstruction, seeding, image after 1 run

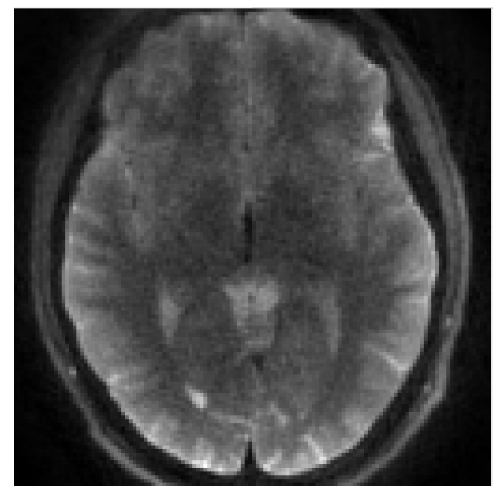

(f) Linear reconstruction, seeding, image after 1 run

Figure 27 - Reconstruction results for nonlinear and subsequent linear reconstruction of b0 images, after repetitive runs of the nonlinear reconstruction step, with and without seeding.

Coil sensitivity regularization The images and coil sensitivities shown in figure 28 show six slices from a dataset of the brain acquired with an isotropic resolution of $1.8 \mathrm{~mm}$. Without a regularization of coil sensitivities over the slices, non-linear inverse reconstruction of this dataset leads to a reconstruction artifact in one slice, with a locally negligible magnitude in the image as well as the coil sensitivities. Moreover, the image as well as the coil sensitivities have a phase singularity in that location. Regularization of the coil sensitivities with a slice regularization strength of $d=1$ yields an image and coil sensitivities without this artifact. Nevertheless, this regularization retains the differences in the coil sensitivities of the different slices. So far, the reconstruction artifact described above has never been observed on datasets which were reconstructed with the proposed seeding and coil sensitivity regularization methods.

Convergence of the reconstruction algorithm The reconstructed image intensity for the b0 image as well as the mean diffusion-weighted image were measured as a function of the number of Newton iterations in a region of white brain matter with approximately homogeneous signal. 


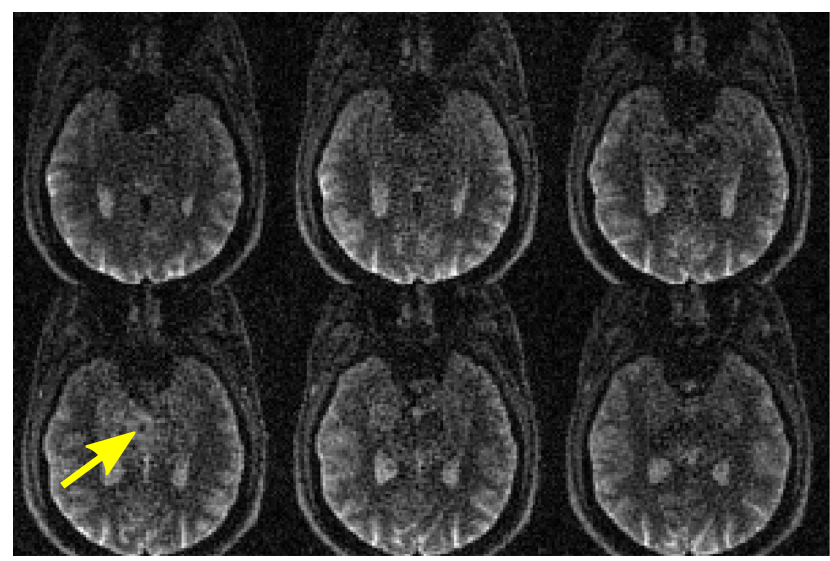

(a) Image content, $\mathrm{d}=0$

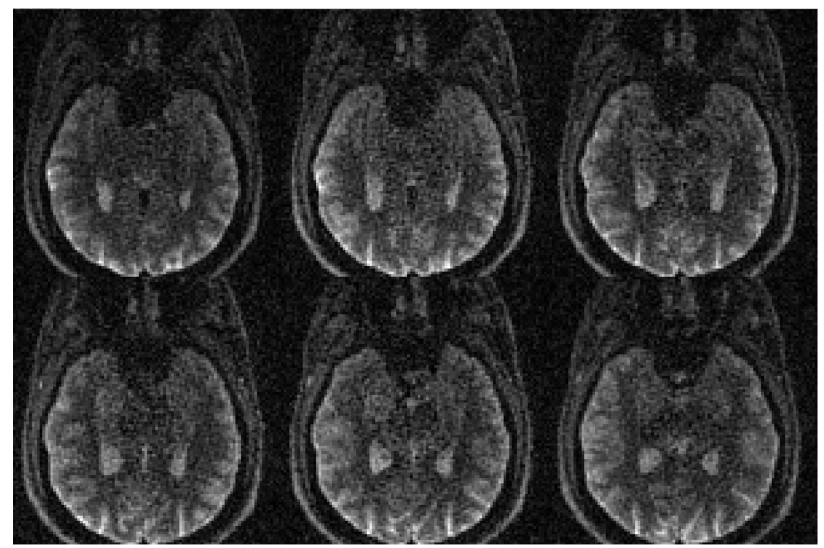

(c) Image content, $\mathrm{d}=1$

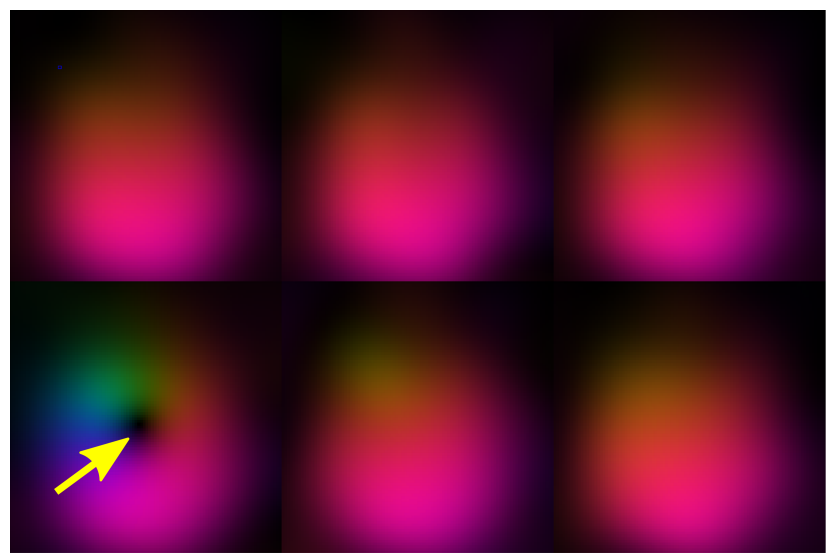

(b) First PC of coil sensitivities, $\mathrm{d}=0$

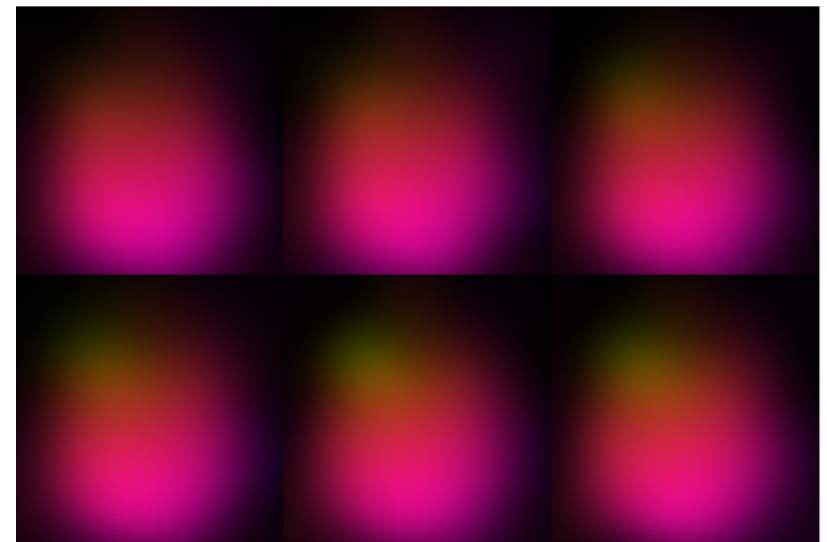

(d) First PC of coil sensitivities, $d=1$

Figure 28 - Suppression of reconstruction errors (marked with arrows) due to coil profile regularization with strength $d$. The complex-valued coil sensitivities are represented using the colour for the phase and the brightness for the magnitude.

The result displayed in figure $29 \mathrm{~b}$ shows a convergent behaviour of the reconstruction algorithm and a constant image intensity for 9 or more Newton iterations. The standard deviations of the intensities in the region increase with more than 9 iterations, which indicates an increase in the noise level. The choice of 9 Newton iterations is confirmed by visual inspection of the corresponding images shown in figure 29, the images (b0 and b1000) reconstructed with 11 Newton iterations appear more corrupted by noise than those reconstructed with 9 Newton iterations. However, a reconstruction with 7 Newton iterations leads to less spatial acuity. 


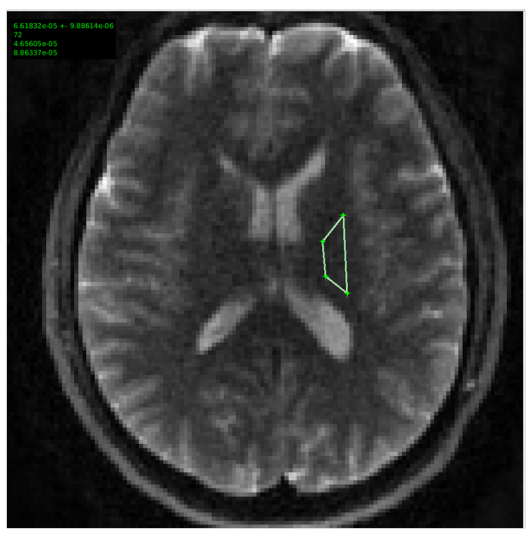

(a) Region used for evaluation of convergence

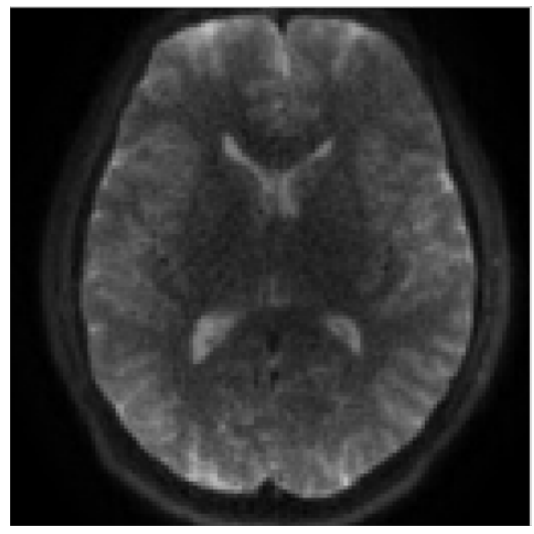

(c) b0, 7 iterations

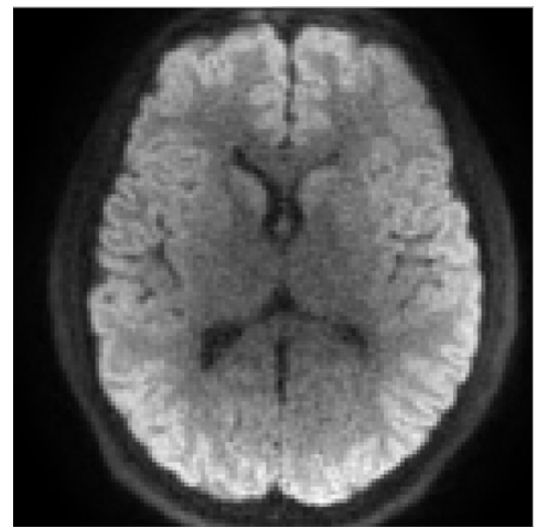

(f) b1000, 7 iterations

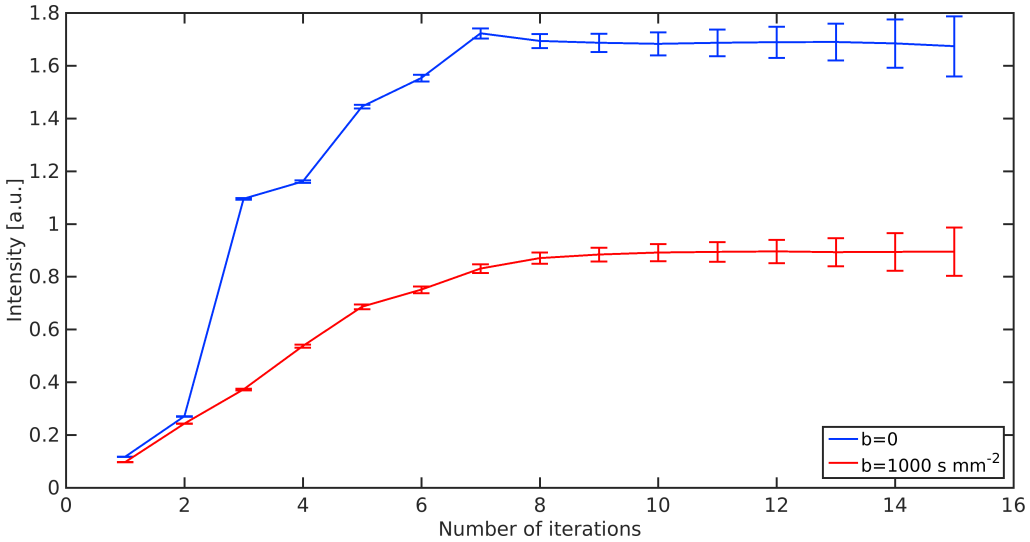

(b) Reconstructed image intensity in the region shown in figure $29 \mathrm{a}$.

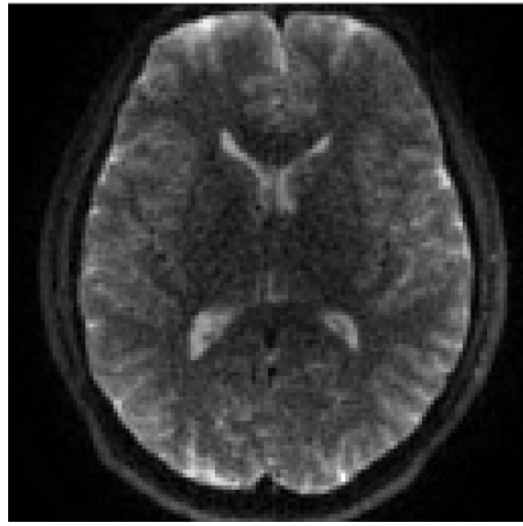

(d) b0, 9 iterations

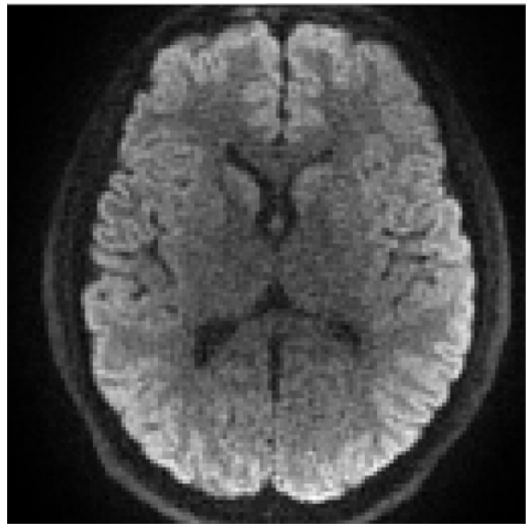

(g) b1000, 9 iterations

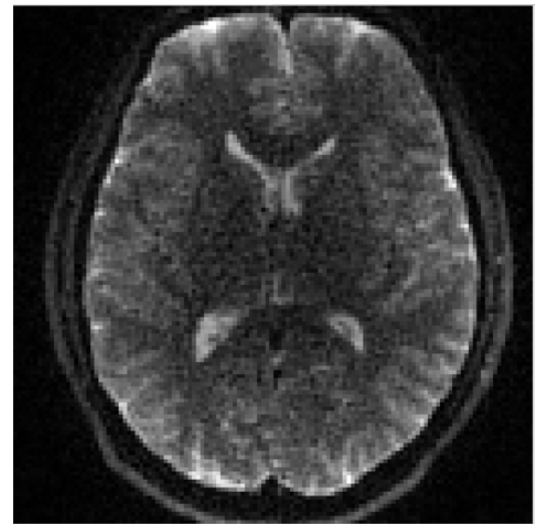

(e) b0, 11 iterations

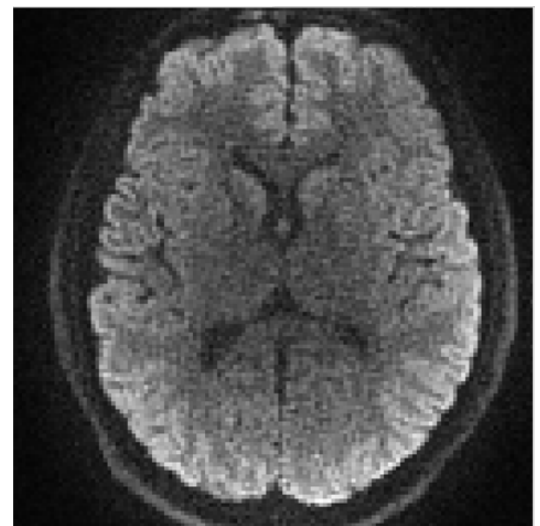

(h) b1000, 11 iterations

Figure 29 - Image quality and regional intensity as a function of the number of Newton iterations. The b-values are given in units of $\mathrm{smm}^{-2}$. The images shown for b1000 are averages of magnitudes over 6 directions. 


\subsubsection{Discussion}

Slice-based regularization of the coil sensitivities led to the avoidance of reconstruction errors with a "black hole" in the image and phase singularities in the coil sensitivities. However, it is likely that the avoidance of such "black hole" artifacts is in part due to the new initialization, since a correct initial estimation of phase maps should reduce the likelihood for the reconstruction algorithm to converge to a cost function minimum with an incorrect phase singularity. Recently, Wang et al [66] observed the removal of the same artifact in a time series of cardiac FLASH MR images by estimating the coil sensitivities from a larger number of frames. This is consistent with the results reported in this thesis, since the proposed regularization effectively increases the amount of data from which every set of coil sensitivities is estimated. An alternative method for the avoidance of such black holes is the ENLIVE (Extended Non-Linear InVersion inspired by ESPIRiT) algorithm which was recently published by Holme et al. [28], where multiple sets of coil sensitivities are reconstructed with a symmetry-breaking constraint which effectively penalizes the formation of a black hole.

Besides providing an initial estimate of the phase distributions for the coil sensitivities, the seeding was shown to accelerate the convergence of the nonlinear reconstruction, in particular with regard to the scaling of intensity values of both the image content and the coil sensitivities. Since convergence of this scaling is necessary to avoid an unpredictable degree of image regularization in the final result, the practical effect of the proposed seeding method is at least a 5-fold reduction of the computation time for the nonlinear reconstruction step, which is crucial for any application in clinical practice. Without the proposed initialization, the relative intensity scaling of image content and coil sensitivities converges much slower than the relative intensity distribution within the image. This can be attributed to the fact that this scaling is determined by the regularization term only.

\subsection{In vivo studies using optimized imaging protocols}

\subsubsection{Methods}

Based on experimental in-vivo optimization of acquisition and image reconstruction parameters, two protocols for rapid diffusion-weighted imaging (DWI) of the entire brain were developed for possible clinical use: "fast DWI" at an in-plane resolution of $1.5 \mathrm{~mm}$, a slice thickness of $4 \mathrm{~mm}$ and a total acquisition time of $84 \mathrm{~s}$, and "high-resolution DWI" at an in-plane resolution of $1.25 \mathrm{~mm}$, a slice thickness of $3 \mathrm{~mm}$ and an acquisition time of $168 \mathrm{~s}$. The acquisition and reconstruction parameters of these protocols are shown in table 1 .

These imaging protocols were tested for robustness to image artifacts on ten volunteers. Moreover, the performance of the high-resolution protocol was tested on two patients in a preliminary trial. 


\begin{tabular}{|c|c|c|}
\hline Sequence & Fast DWI & High-resolution DWI \\
\hline \hline In-plane resolution $[\mathrm{mm}]$ & 1.5 & 1.25 \\
\hline Matrix size & 128 & 152 \\
\hline Field of view [mm] & 192 & 190 \\
\hline Slice thickness [mm] & 4 & 3 \\
\hline Max. number of slices & 55 & 54 \\
\hline b-value [s $\left.\mathrm{mm}^{-2}\right]$ & 1000 & 1000 \\
\hline No. of averages & 1 & 2 \\
\hline Diffusion directions & 6 & 6 \\
\hline TR [ms] & 12000 & 12000 \\
\hline Total acquisition time & $84 \mathrm{~s}$ & $168 \mathrm{~s}$ \\
\hline Bandwidth [Hz/Pixel] & 500 & 500 \\
\hline Radial spokes & 35 & 35 \\
\hline TSE [ms] & 35.5 & 35.7 \\
\hline TR $\alpha[\mathrm{ms}]$ & 4.41 & 4.54 \\
\hline TE [ms] & 5.36 & 5.54 \\
\hline Newton iterations & 9 & 9 \\
\hline Slice regularization strength & 1 & 1 \\
\hline
\end{tabular}

Table 1 - Optimized imaging protocols.

\subsubsection{Results}

Figure 30 shows the previous state-of-the-art image quality [36] for diffusion-weighted STEAM MRI with cartesian sampling and image reconstruction using the Generalized Autocalibrating Partial Parallel Acquisition (GRAPPA) algorithm [18]. The total acquisition time for these data sets was $330 \mathrm{~s}$, with an in-plane resolution of $1.44 \mathrm{~mm}$, a slice thickness of $5 \mathrm{~mm}$, and a b-value

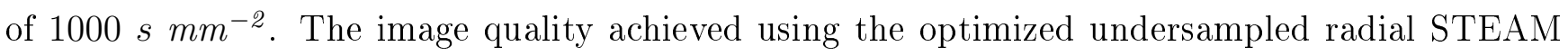
DWI protocols (table 1) is shown in figure 31 for the fast DWI protocol, and in figure 32 for the high-resolution DWI protocol. Similar image quality was achieved in 10 volunteers on which these protocols were tested. In particular, no susceptibility artifacts and none of the image artifacts shown in sections 4.3 and 4.4 .2 were observed in this study with either of the two optimized protocols.

Comparison of the mean diffusion-weighted images with the STEAM DWI results from figure 30 show that undersampled radial STEAM DWI with iterative image reconstruction yield visually superior image quality as characterized by spatial acuity and contrast between gray and white matter compared to the results published by Khalil et al. This was achieved with comparable voxel volumes and a 4-fold reduction in acquisition time (fast DWI) or, for high-resolution DWI, a 2 -fold reduction in both acquisition time and voxel volume. SNR quantification for the b0 images acquired with high-resolution STEAM DWI yielded inconsistent results for two tested methods. Using the same method as Khalil et al. [36], with an estimation of the noise level from the standard 


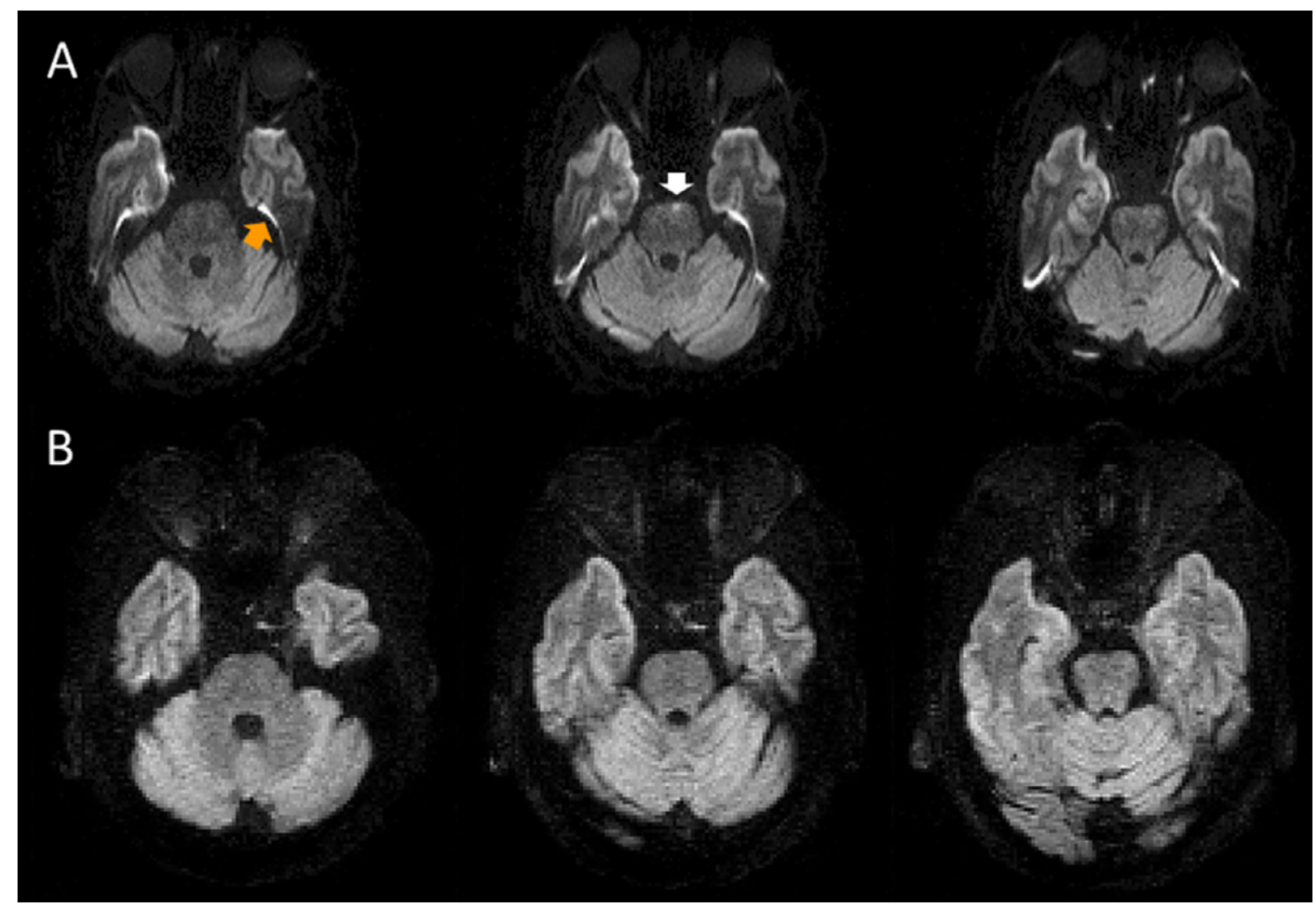

Figure 30 - Previous state-of-the-art image quality for EPI (row A) and STEAM DWI (row B). Reprint from ref. [36], fig. 2. This result shows susceptibility-induced artifacts marked with arrows, which are avoided with use of the STEAM DWI sequence.

deviation of the intensity in a region outside the brain, the result was an SNR of 87 . The "difference method", where the noise level is estimated from the difference between two identical subsequent acquisitions, resulted in an SNR estimate of 20.

The ADC values measured in the centrum semiovale, a large region containing white brain matter (see figure 33) for the 10 volunteers yielded ADC values of $(683 \pm 18) \cdot 10^{-6} \mathrm{~mm}^{2} \mathrm{~s}^{-1}$ (fast DWI) and $(680 \pm 24) \cdot 10^{-6} \mathrm{~mm}^{2} \mathrm{~s}^{-1}$ (high-resolution DWI) which are in agreement with each other and with the range of literature values of $(687 \pm 23) \cdot 10^{-6} \mathrm{~mm}^{2} \mathrm{~s}^{-1}[29],(703 \pm 36) \cdot 10^{-6} \mathrm{~mm}^{2} \mathrm{~s}^{-1}$ [7] and $(736 \pm 40) \cdot 10^{-6} \mathrm{~mm}^{2} \mathrm{~s}^{-1}$ [30].

The STEAM DWI results from preliminary trials on one patient with subacute lacunar stroke and one patient with an epidermoid cyst are displayed in figure 34. These images provide relevant diagnostic information which is not corrupted by any susceptibility artifacts. The hindered diffusion caused by the stroke can be located very well in the mean diffusion-weighted STEAM image. DWI also allows a detailed characterization of the inner structure of the cyst, which appears homogeneous in the non-diffusion-weighted image. The diffusion-weighted image shows dark regions, which contain fluid, as well as bright regions of jelly-like compartments. 


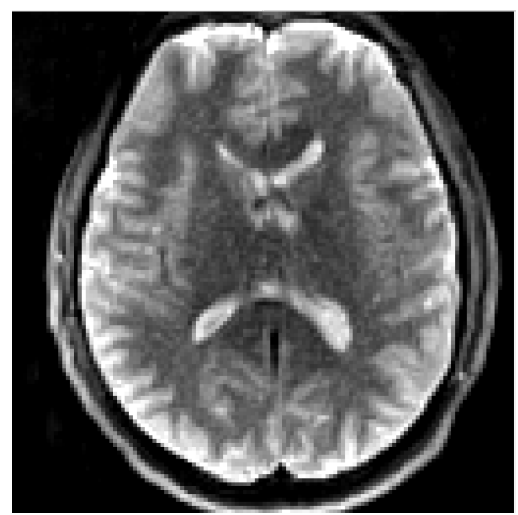

(a) b0, upper slice

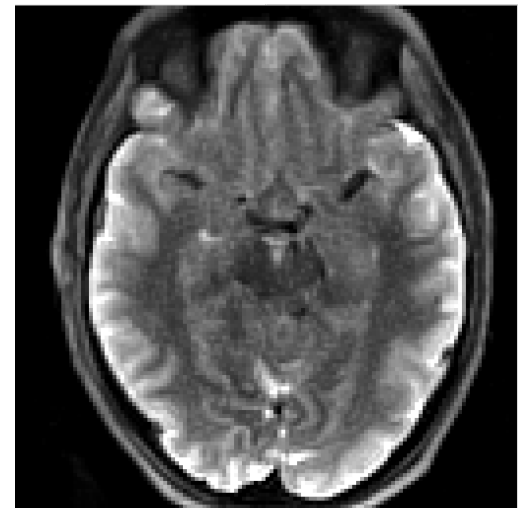

(d) b0, middle slice

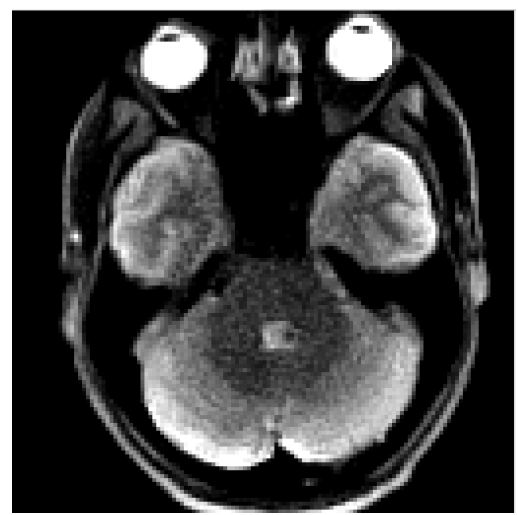

(g) b0, lower slice

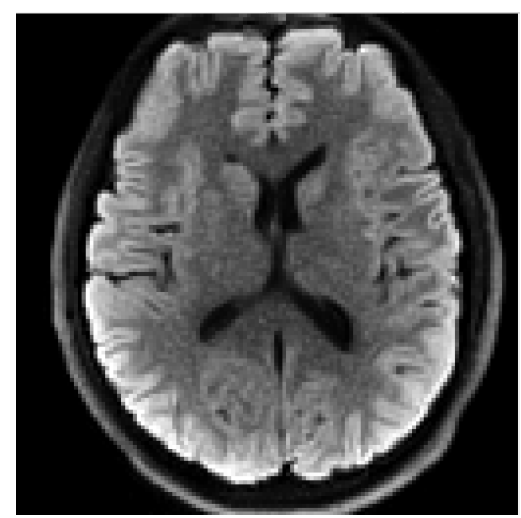

(b) b1000, upper slice

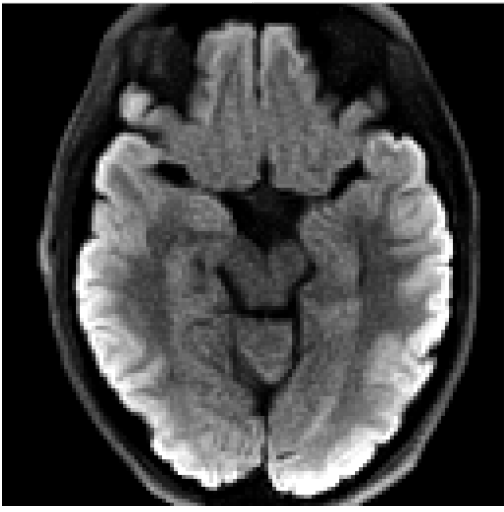

(e) b1000, middle slice

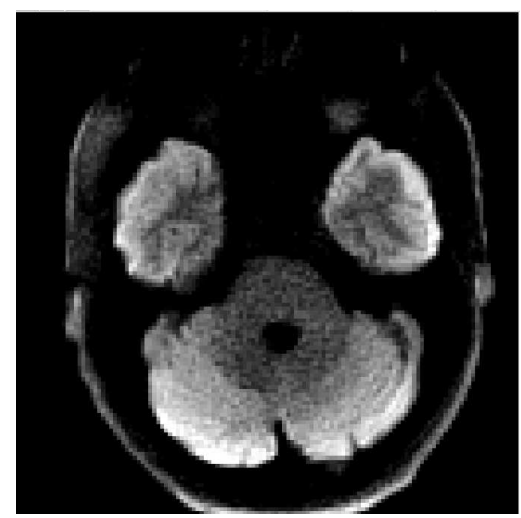

(h) b1000, lower slice

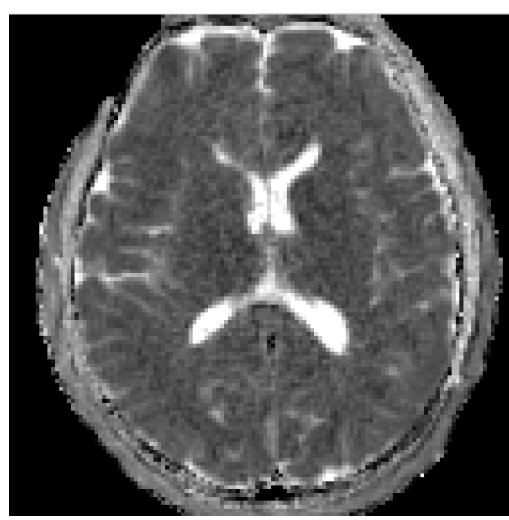

(c) ADC, upper slice

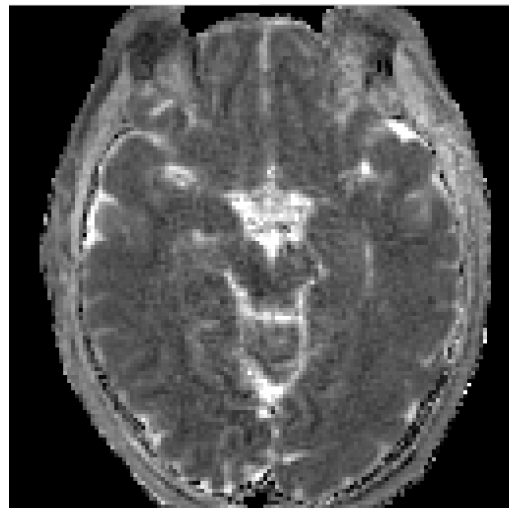

(f) ADC, middle slice

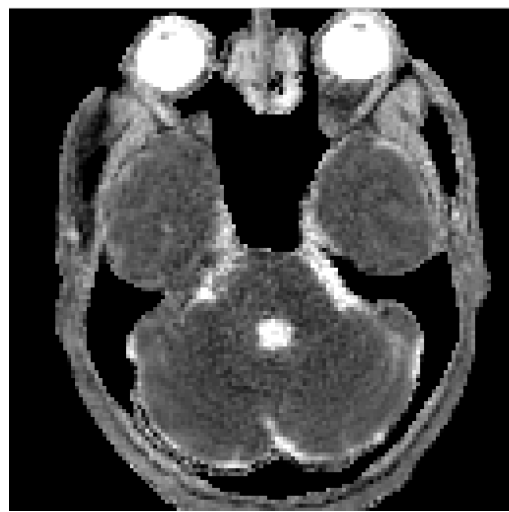

(i) ADC, lower slice

Figure 31 - Image quality obtained with fast diffusion-weighted imaging protocol. The b-values are given in units of $s \mathrm{~mm}^{-2}$. The images shown for b1000 are averages of magnitudes over 6 directions. 


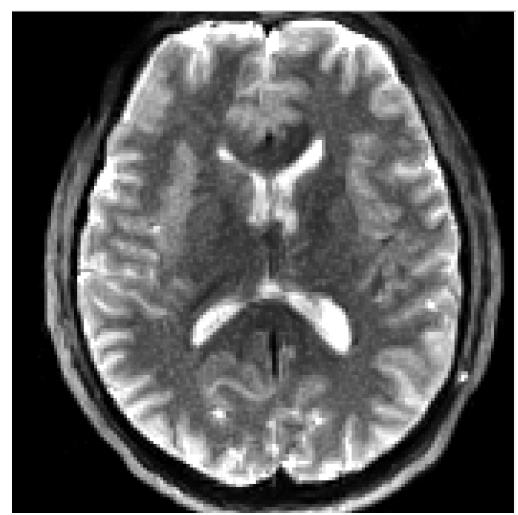

(a) b0, upper slice

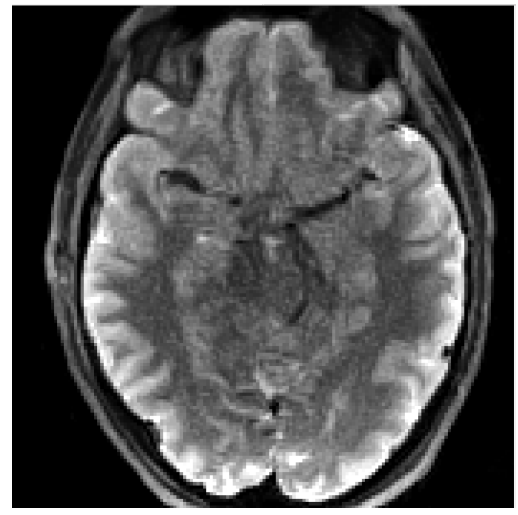

(d) b0, middle slice

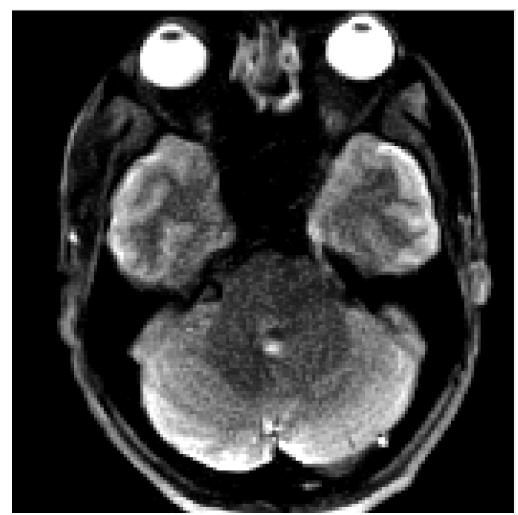

(g) b0, lower slice

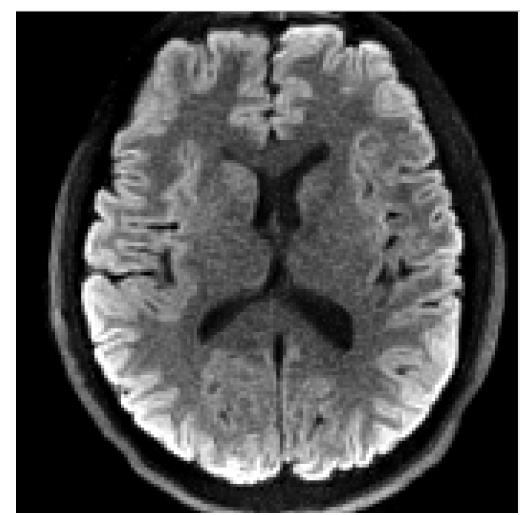

(b) b1000, upper slice

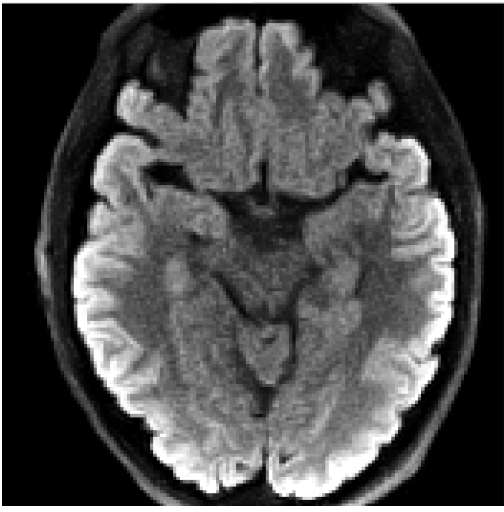

(e) b1000, middle slice

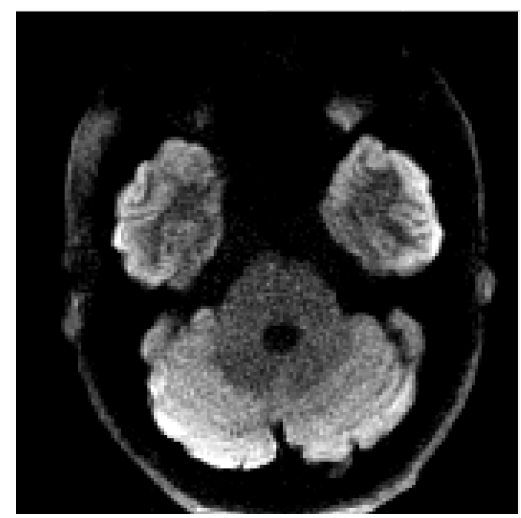

(h) b1000, lower slice

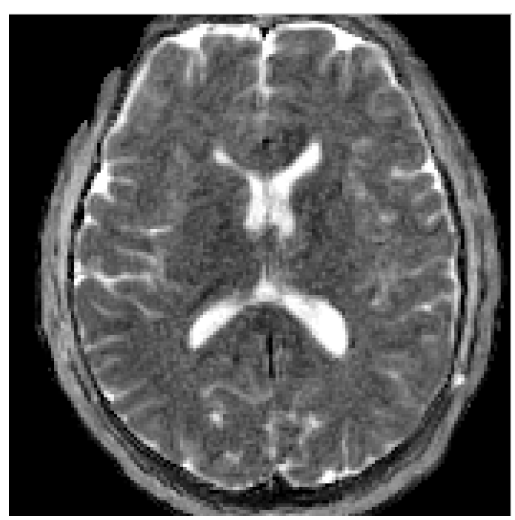

(c) ADC, upper slice

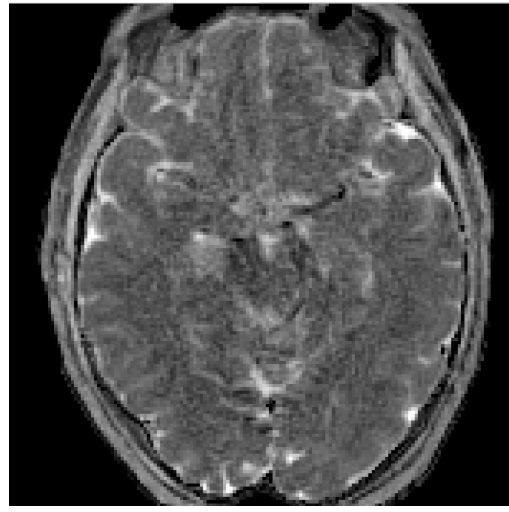

(f) ADC, middle slice

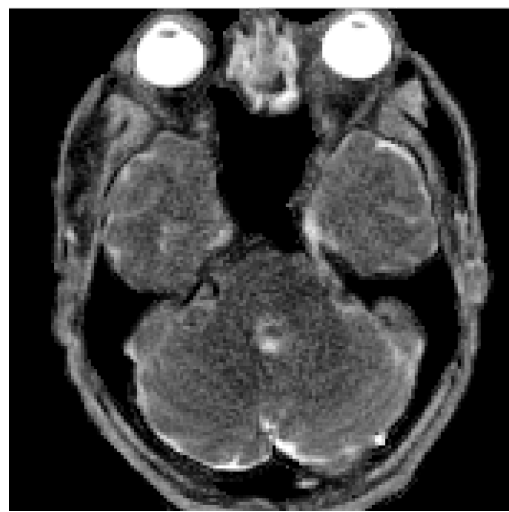

(i) ADC, lower slice

Figure 32 - Image quality obtained with high-resolution diffusion-weighted imaging protocol. The b-values are given in units of $s \mathrm{~mm}^{-2}$. The images shown for b1000 are averages of magnitudes over 6 directions. 


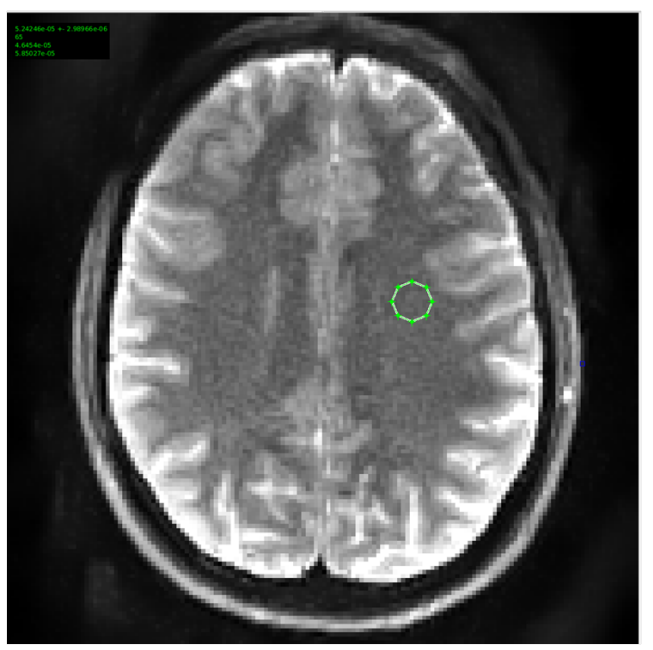

Figure 33 - Region of interest in the centrum semiovale for the validation of ADC measurements.

\begin{tabular}{|c|c|c|}
\hline Subject & Fast DWI & High-resolution DWI \\
\hline \hline$\# 1$ & $680 \pm 12$ & $656 \pm 8$ \\
\hline$\# 2$ & $715 \pm 10$ & $682 \pm 9$ \\
\hline$\# 3$ & $684 \pm 10$ & $698 \pm 8$ \\
\hline$\# 4$ & $665 \pm 10$ & $664 \pm 9$ \\
\hline$\# 5$ & $656 \pm 10$ & $645 \pm 8$ \\
\hline$\# 6$ & $687 \pm 9$ & $669 \pm 8$ \\
\hline$\# 7$ & $673 \pm 10$ & $689 \pm 8$ \\
\hline$\# 8$ & $680 \pm 10$ & $709 \pm 8$ \\
\hline$\# 9$ & $682 \pm 9$ & $666 \pm 9$ \\
\hline$\# 10$ & $708 \pm 9$ & $718 \pm 8$ \\
\hline Mean \pm SD & $683 \pm 18$ & $680 \pm 24$ \\
\hline
\end{tabular}

Table 2 - ADC values in the centrum semiovale $\left[10^{-6} \mathrm{~mm}^{-2} \mathrm{~s}^{-1}\right]$ 


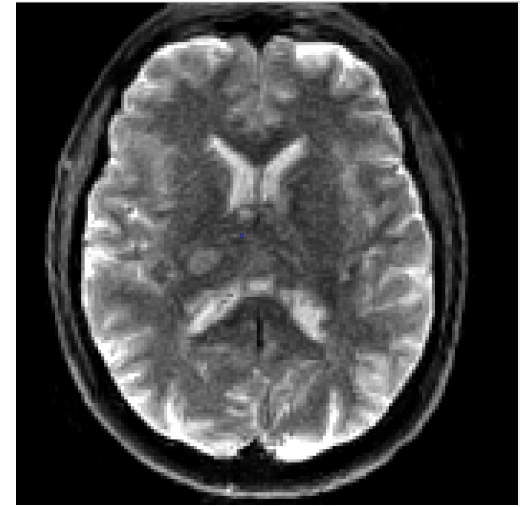

(a) b0, stroke

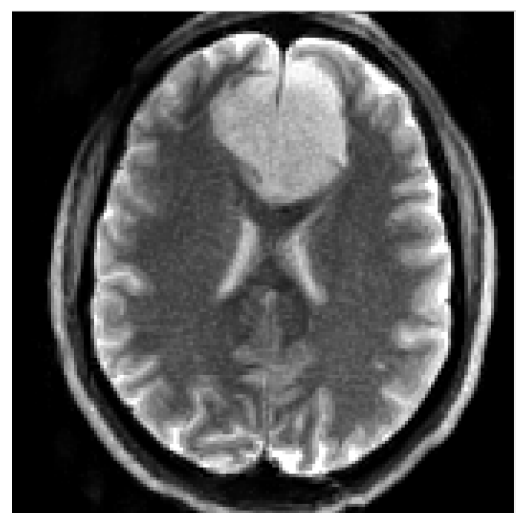

(d) b0, cyst

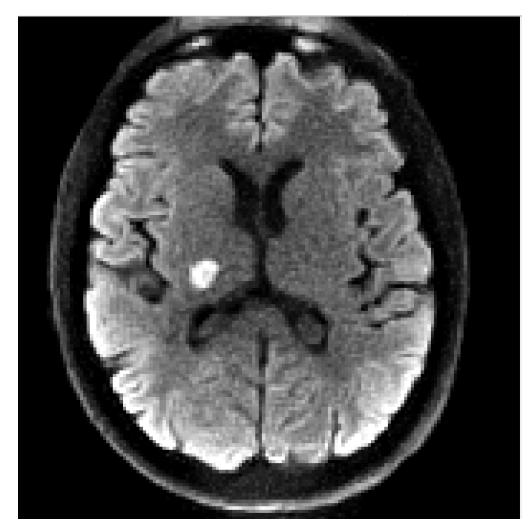

(b) b1000, stroke

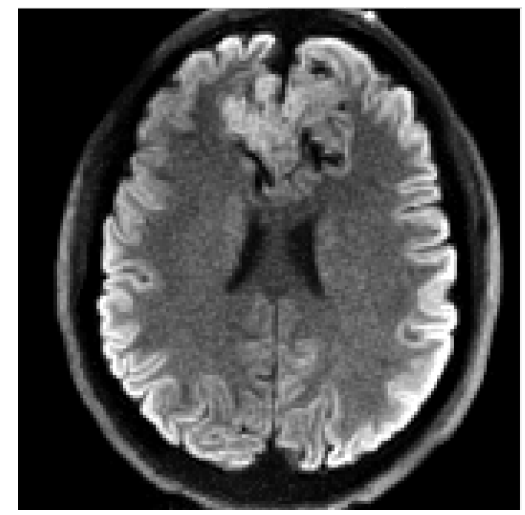

(e) b1000, cyst

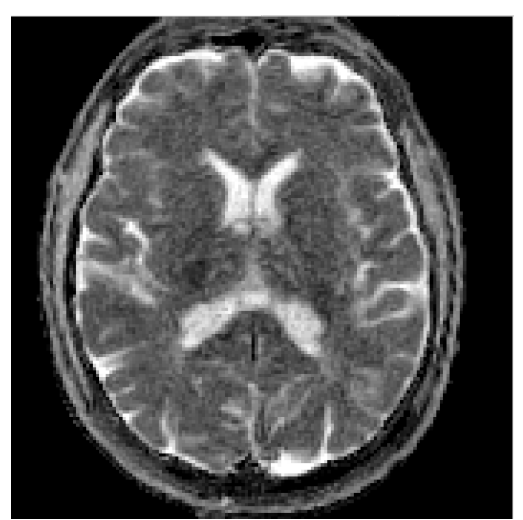

(c) ADC, stroke

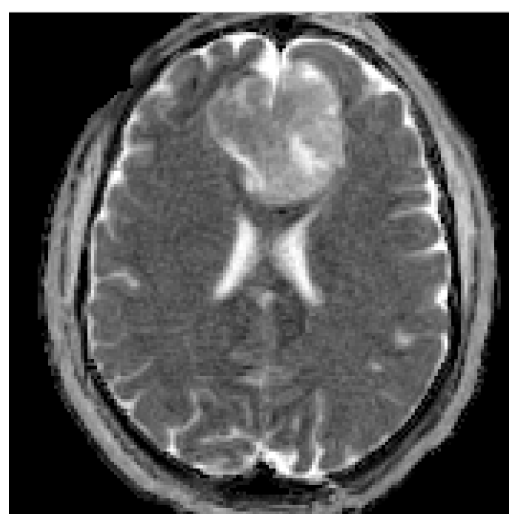

(f) ADC, cyst

Figure 34 - High-resolution DWI applied to patients with a lacunar stroke (upper row) and an epidermoid cyst (lower row). The restricted diffusion for stroke is well seen in the diffusionweighted (b1000) image (b). The diffusion-weighted image of the cyst (e) details its internal structure which contains fluid (dark) and jelly-like (bright) compartments. The b-values are given in units of $s \mathrm{~mm}^{-2}$. The images shown for b1000 are averages of magnitudes over 6 directions. 


\subsection{Discussion}

This thesis reports the first successful development and application of a diffusion-weighted singleshot STEAM MRI technique with radial sampling of k-space. Two imaging protocols were proposed, one emphasizing spatial resolution, the other emphasizing low scan times. These protocols allow diffusion-weighted imaging in clinically feasible scan time, resolution and image quality, which was reproducible in 10 tested volunteers.

To achieve this result, the new developments in the image acquisition and reconstruction technique were crucial. The modified variable flip angle model avoided local streaking artifacts with minimal SNR loss, and stable image reconstruction was achieved with a combination of regularization of the coil sensitivities across the slices and a new method of data scaling and initialization.

The benefits of a radial sampling trajectory and NLINV-based image reconstruction for DWSTEAM imaging can be understood by comparison with the latest methodological publication on cartesian DW-STEAM of the brain by Karaus et al. [33]. This technique uses partial Fourier imaging, i.e. the exploitation of the symmetries of the Fourier transform, and parallel imaging with GRAPPA reconstruction [18] to acquire images with an undersampled cartesian trajectory of 31 lines, whereas the radial STEAM protocols use 35 spokes. However, the in-plane resolution for the cartesian sequence was $1.8 \mathrm{~mm}$, with a total of 84 pixels in the phase encoding direction, versus $1.25 \mathrm{~mm}$ resolution and $152 \times 152$ pixels for the undersampled radial high-resolution DWI protocol. Hence, the use of a radial trajectory with NLINV-based reconstruction increased the undersampling factor from 2.7 to 4.3. This increase of the undersampling factor was possible mainly due to the superior performance of NLINV compared to GRAPPA for undersampled kspace trajectories [60, but also the favorable undersampling properties of radial trajectories due to a high sampling density in the center of k-space. With an undersampling factor of 2.7, the number of radial spokes would be 56 instead of 35. This would reduce the maximum number of acquired slices for high-resolution DWI at $\mathrm{TR}=12 \mathrm{~s}$ from 54 to 38 . In-vivo experiments have shown that this would be insufficient for reliable whole-brain coverage, which requires 47 slices of 3 mm thickness. This proves that the proposed image acquisition and reconstruction technique has considerably reduced the scan time for whole-brain DWI at the resolution defined by the proposed high-resolution protocol. Imaging protocols with even higher spatial resolution would benefit even more from the improved undersampling properties, because the scan time would be defined only by the duration of the pulse sequence itself and not by an optimization of the image contrast and SNR which is affected by T1 relaxation for short repetition times. An additional benefit of accelerated acquisition of each slice is that signal losses due to T1 relaxation between the second $90^{\circ}$ pulse and the readout pulses is reduced, while the SNR loss due to a lower number of radial spokes is compensated by the higher SNR per spoke due to higher flip angles.

Comparison of the results of the described radial single-shot STEAM technique to the recently

published results by Khalil et al. for cartesian DW-STEAM imaging clearly show a major ad- 
vancement. The image quality is arguably improved while the scan time is either reduced by a factor of 4 at comparable spatial resolution (fast DWI) or by a factor of 2 at half the voxel volume (high-resolution DWI). Exact quantification of this improvement in terms of SNR is difficult because Khalil et al. have calculated the SNR by estimating the noise level from a background region. Apart from the fact that this method is generally compromised by high uncertainties [9], the reconstruction method proposed in this thesis does not produce homogeneous background noise. Therefore, the calculated SNR improvement from 36 (Khalil et al.) to 87 probably overestimates the real SNR increase. On the other hand, the difference method [9] which yielded an SNR of 20 for high-resolution DWI systematically underestimates the SNR, because motion and brain pulsations are included in the difference image and lead to an overestimated noise level. Therefore, a meaningful quantitative value of the relative increase in SNR per measuring time due to the new methodological developments could not be computed; based on the two diverging estimates, the factor should be between 2 and 9 .

The observed improvement of SNR efficiency can be explained by a combination of the effects of methodological developments and improved hardware.

The Siemens Tim Trio 3T scanner used by Khalil et al. provides a maximal gradient strength of $40 \frac{m T}{m}$, whereas the Siemens Prisma 3T scanner used for the experiments described in this thesis allows for $80 \frac{m T}{m}$. This has reduced the spin echo time from $44 \mathrm{~ms}$ [36] to $35.7 \mathrm{~ms}$. For white matter with $T 2=80 \mathrm{~ms}$ [67, this results in $10 \% \mathrm{SNR}$ gain, for gray matter with $\mathrm{T} 2=110 \mathrm{~ms}$, the SNR gain is 7\%. Another possible source of SNR increase is the use of a 64-channel head coil for signal acquisition for this work, whereas Khalil et al. used a 12-channel head coil.

Besides some SNR improvement due to improved undersampling properties, two major factors in the methodological developments which increase the SNR can be identified.

Firstly, the $L_{2}$ regularization term in the reconstruction algorithm suppresses image noise. Compared to image reconstruction by gridding and fast Fourier transforms (FFT), the improved SNR in nonlinear inversion with $L_{2}$ regularization can clearly be seen in the cardiac images in section 3. Even though no equivalence between the gridding reconstruction and the GRAPPA reconstruction used for cartesian STEAM data can be assumed, the $L_{2}$ regularization term in NLINV should also lead to an improvement in SNR in comparison to GRAPPA reconstruction, which does not include any regularization. Ref. [60] provides a direct comparison of images reconstructed with GRAPPA and NLINV from the same data, which supports this claim.

Secondly, the application of the non-local means filter improves the SNR as estimated using the difference method by a factor of 2 . This is consistent with the theoretical prediction using a filter strength of 0.5 (parameter $\alpha_{\max }$ in ref. [38]). It is, however, unclear if the images presented by Khalil et al. were processed with an image filter provided by the vendor of the MRI device, which was not explicitly mentioned in their clinically oriented publication.

For a quantification of the SNR improvement achieved by the proposed imaging technique excluding effects of different hardware, and of the influence which each of the described effects have 
on image SNR, a re-development of cartesian STEAM DWI and GRAPPA image reconstruction as used by Khalil et al. would be necessary. This was beyond the scope of this work. Moreover, both experimental results and theoretical considerations show that inferior results compared to the proposed method are to be expected. Hence, such an extensive re-development project would not be of any practical use beyond a more quantitative description of the improvements of the SNR due to the effects of radial undersampling, iterative image reconstruction and denoising as described above.

The described imaging technique is the first implementation of single-shot STEAM DWI which yields diffusion-weighted images of the brain with clinically feasible scan time, resolution and image quality. First results on patients as shown in this thesis are promising and motivate further clinical studies on the performance of this technique in diagnostic brain MRI. The following section describes the application of STEAM DWI to prostate imaging, where the benefit of avoiding susceptibility artifacts is even greater than in brain imaging. 


\section{Diffusion-weighted STEAM MRI of the prostate}

\section{$5.1 \quad$ Introduction}

Prostate cancer is the most common form of cancer which affects adult males. Diffusion-weighted MRI is a potentially useful tool for detection and classification of lesions as well as assessment of treatment response [54]. However, diffusion-weighted EPI of the prostate is prone to major susceptibility-induced artifacts which can lead to image distortions [42] as well as local errors in the ADC maps which may lead to false diagnosis [54]. This is due to the sharp local inhomogeneities of the magnetic susceptibility in the vicinity of the prostate, in particular at the boundary to the colon which may be filled with air (see figure 35). Thus, the single-shot STEAM sequence is a potentially useful technique for prostate DWI. In this chapter, the first application of the diffusion-weighted STEAM sequence presented in section 4.2 to prostate imaging is described.

To develop an imaging protocol which is competitive with current clinical practice, imaging parameters were adapted to those of the clinical prostate DW-EPI protocol developed by Siemens for imaging with the Magnetom Prisma (3T) MRI device and a body-array coil. This commercial EPI protocol uses an in-plane resolution of $1.43 \mathrm{~mm}$, a slice thickness of $3.5 \mathrm{~mm}$, a maximal b-value of $800 \mathrm{~s} \mathrm{~mm}^{-2}$ and a total acquisition time of $4 \mathrm{~min}$.

The use of the body-array coil and the spine coil for image acquisition in the prostate generally leads to lower SNR compared to brain imaging with the 64-channel head coil. This is caused by the greater distance between the prostate and the coils, which are situated below the table carrying the

patient (spine coil) or fixed around the patient's hips (body-array coil). Shorter distances between the prostate and the receive coils are possible using endorectal coils, but only at the cost of major inconvenience for the patient and a risk of poor patient compliance, and little chance of testing any novel imaging techniques on healthy volunteers. The study of DW MRI of the prostate for this work is therefore limited to image acquisition with a body-array coil and spine coil.

The low SNR of the acquired data has two important consequences. Firstly, averaging over multiple acquisitions is necessary to achieve adequate SNR. Secondly, averaging over magnitude images, as done for high-resolution brain DWI, leads to a systematic underestimation of the ADC. To avoid both ADC underestimation which results from magnitude averaging, and the errors caused by complex averaging due to inconsistent motion-associated phases, a multi-shot imaging imaging approach was developed, which is described in the following section. 


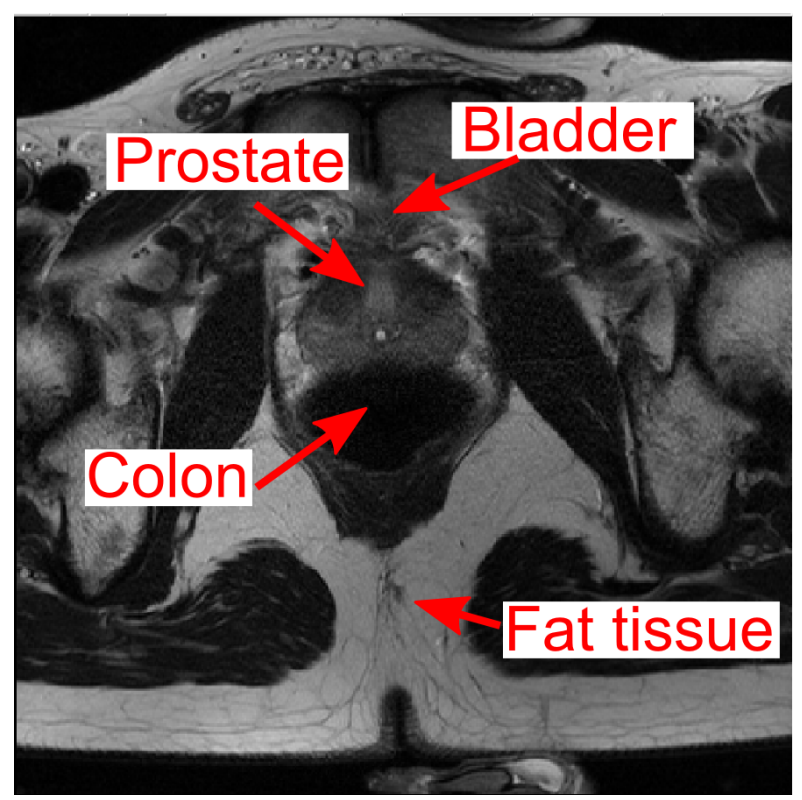

(a) Transverse slice with prostate

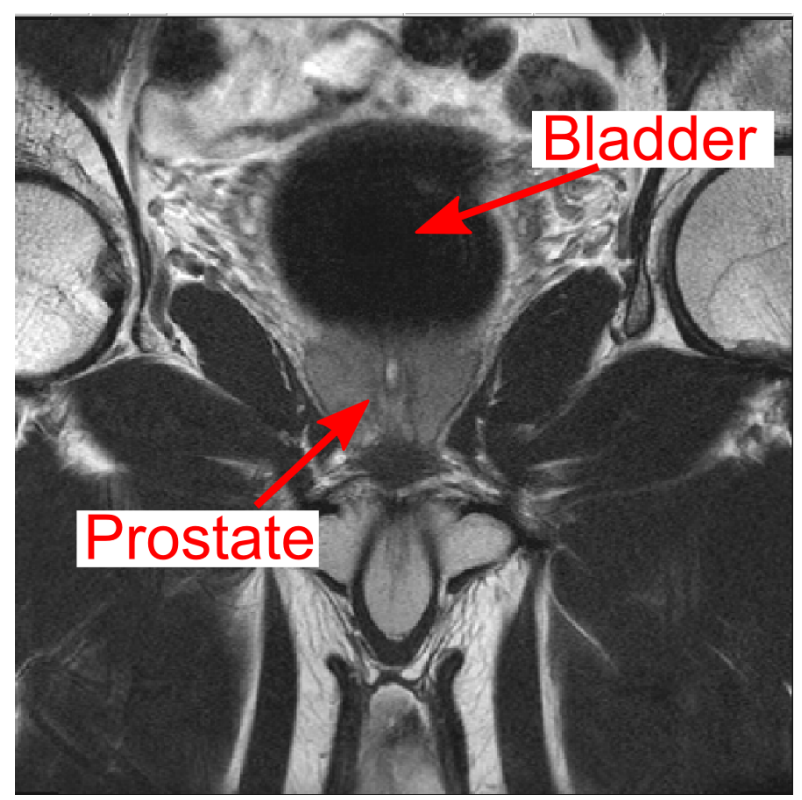

(b) Coronal slice with prostate

Figure 35 - Anatomical location of the prostate and surrounding tissue

\subsection{Methods}

\subsubsection{ADC measurements from data with low SNR}

The effect of low SNR on the measurement of the ADC can be understood with the following calculation: we assume a true image intensity $S_{0}$ for $b=0$ and $S_{1}$ for the diffusion-weighted image, both real and positive. The true diffusion coefficient $D=\frac{1}{b} \ln \left(\frac{S_{0}}{S_{1}}\right)$. If both the real and the imaginary part of $S_{0}$ and $S_{1}$ are corrupted by Gaussian noise with a standard deviation of $\sigma$, the corresponding expectation values $E\left[S_{0,1}\right]=\int_{z \in \mathbb{C}} \mathcal{N}(z, 0, \sigma) \sqrt{\left(S_{0,1}+\Re(z)\right)^{2}+(\Im(z))^{2}} d z$, with $\mathcal{N}$ the complex-valued normal distribution with a standard deviation of $\sigma$. The estimated diffusion coefficient will tend to $D^{\prime}=\frac{1}{b} \ln \left(\frac{E\left[S_{0}\right]}{E\left[S_{1}\right]}\right)<D$. The resulting systematic relative error $\frac{D-D^{\prime}}{D}$ is shown in figure 36 as a function of $S N R=\frac{S_{0}}{\sigma}$.

\subsubsection{Multi-shot image reconstruction}

To reconstruct diffusion-weighted images and ADC maps while avoiding this systematic error, a multi-shot image reconstruction algorithm was developed. This algorithm is based on the method developed by Uecker et al. for segmented cartesian STEAM DWI [61]. The image content for one diffusion-weighted image is jointly reconstructed from the data of all averages (shots), with precalculated phase maps included in the reconstruction model. The algorithm uses the following steps:

1. Preprocessing and nonlinear inverse reconstruction with coil sensitivity regularization of the 


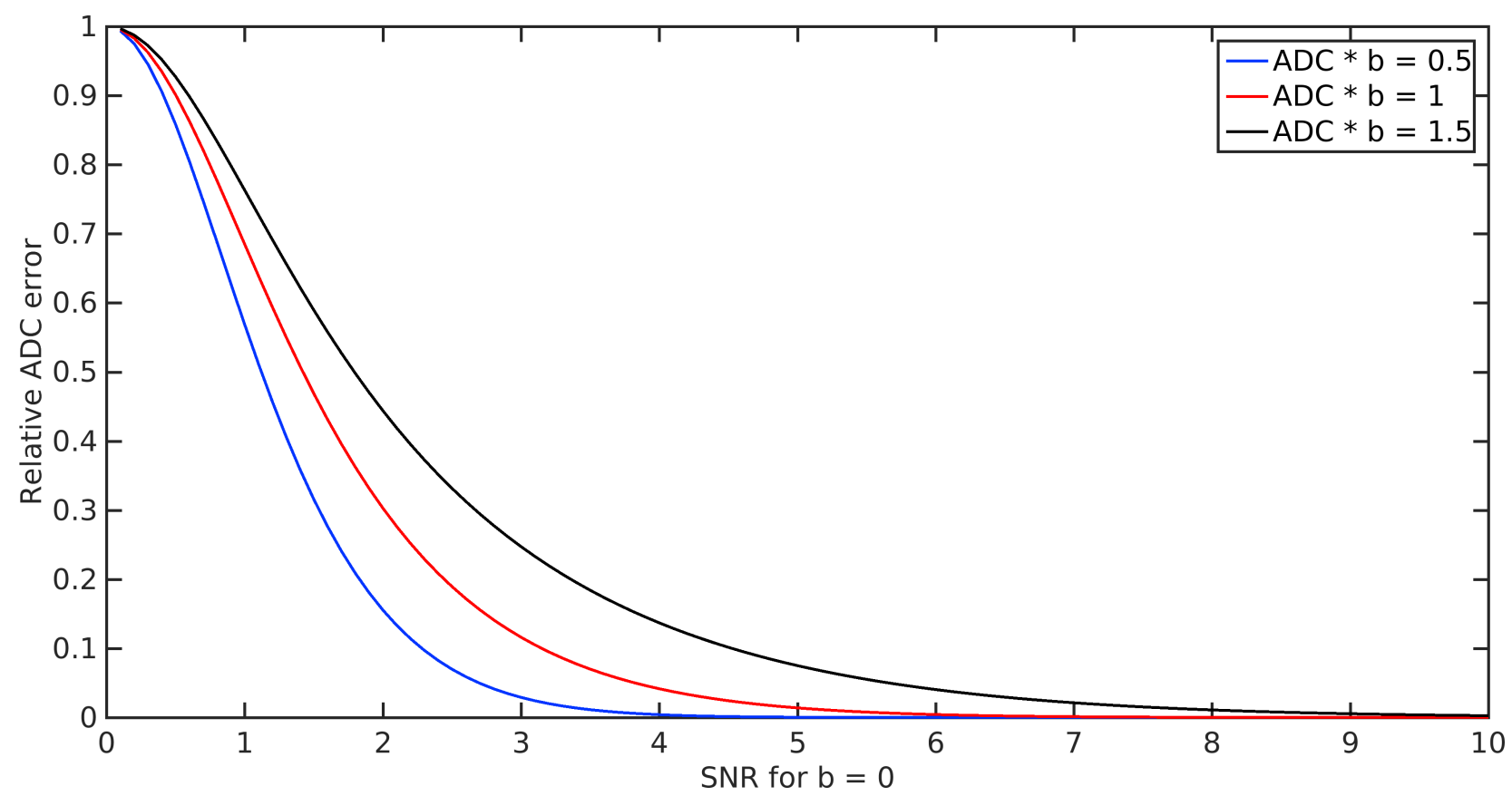

Figure 36 - The systematic relative error of the diffusion coefficient calculated with magnitude averaging as a function of the SNR of the image with $b=0$. In all cases, the estimated diffusion coefficient is lower than the true diffusion coefficient.

entire dataset as described in section 4.4. This results in an image $I_{m}$ for each shot $m=$ $1, \ldots, M$, coil sensitivities $C_{n}$ for virtual channels $n=1, \ldots, N$.

2. Calculation of phase maps $e^{i \varphi_{m}}$. Three different options can be used here:

(a) Calculation without phase map processing: $e^{i \varphi_{m}}=\frac{I_{m}}{\left|I_{m}\right|}$.

(b) Denoising with NLM: $e^{i \varphi_{m}}=\frac{N L M\left(I_{m}\right)}{\left|N L M\left(I_{m}\right)\right|}$.

(c) k-space truncation: as (a), but with the image $I_{m}$ reconstructed from gridded data truncated in $\mathrm{k}$-space (see figure 37). The default truncation factor is 0.2 , this parameter is used unless stated otherwise.

3. Linear multi-shot reconstruction of image content: Minimization of the functional

$$
\sum_{m, n}\left\|Y_{m, n}-P_{m} \mathcal{F}\left(C_{n} e^{i \varphi_{m}} r\right)\right\|_{2}^{2}+\alpha\|r\|_{2}^{2}
$$

with respect to the image content $\mathrm{r}$ using conjugate gradients. The regularization parameter $\alpha$ is the same as for the final Newton iteration in step 1.

4. Post-processing:

(a) Calculation of trace-weighted images for all b-values and denoising with NLM.

(b) Calculation of the ADC map. 
i. Direct calculation for two b-values $b_{1}, b_{2}: A D C=\frac{1}{b_{1}-b_{2}}\left[\ln \left(\operatorname{Tr} a\left(b_{1}\right)\right)-\ln \left(\operatorname{Tr} a\left(b_{2}\right)\right)\right]$, where $\operatorname{Tr} a\left(b_{1,2}\right)$ denotes the trace-weighted images for the two b-values.

ii. For more than two b-values: weighted least squares fit [37].

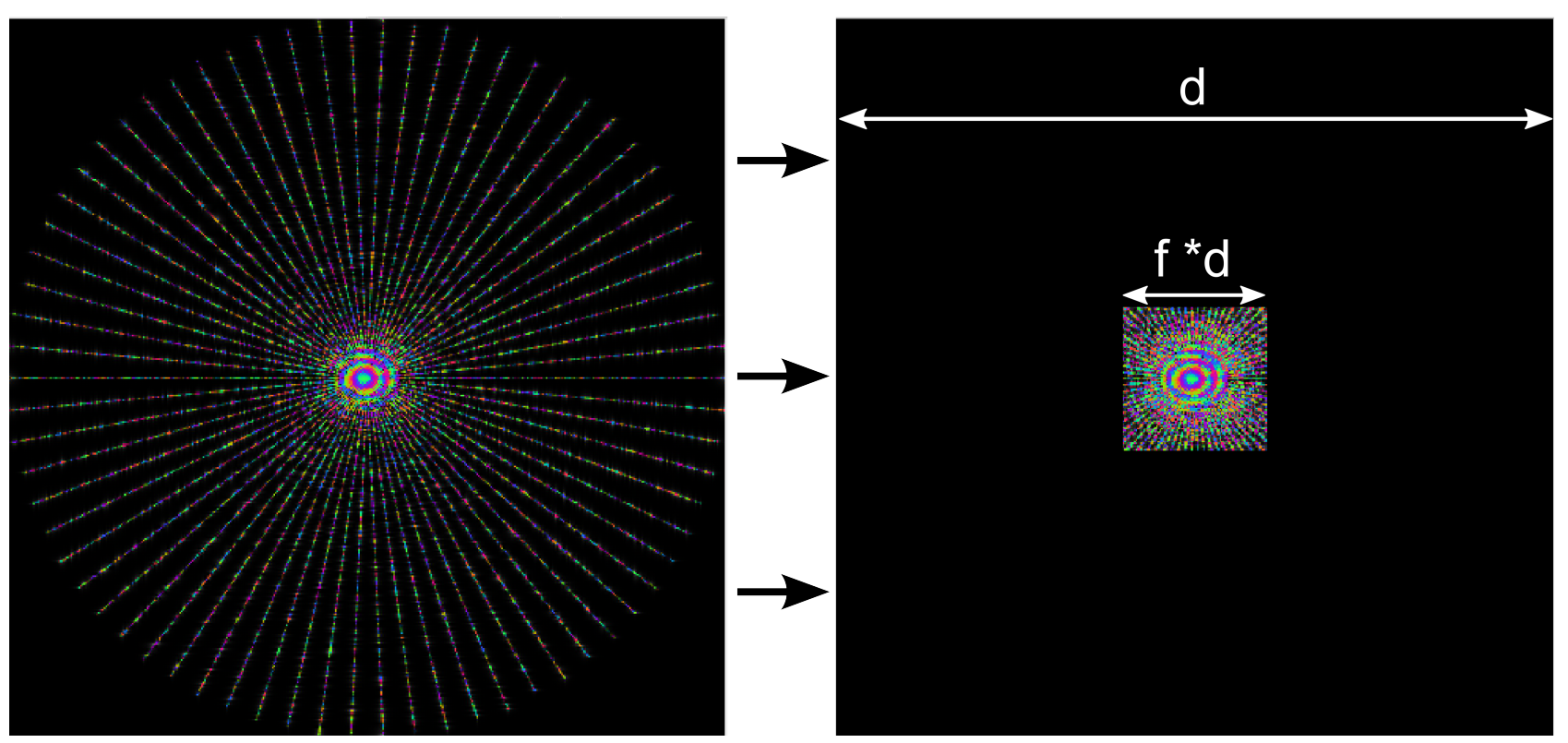

Figure 37 - K-space truncation for the reconstruction of phase maps. The degree of truncation is defined by the factor $\mathrm{f}$, with a default value of 0.2 .

\subsubsection{Validation of the reconstruction algorithm}

This reconstruction method was validated on a numerical phantom consisting of disjoint ellipses which are defined analytically. The non-diffusion-weighted intensity in all ellipses has a magnitude of one. To simulate isotropic diffusion, the diffusion tensor is assumed to be the identity, multiplied by an ADC value predefined for each ellipse. The magnitude of the diffusion-weighted image intensity is chosen according the monoexponential diffusion model. To simulate the effect of unpredictable bulk motion, a complex phase is chosen randomly for each ellipse, each diffusion weighting and each shot. The geometry and the ADC values of the phantom are shown in figure 38 .

Numerical experiments were performed with experimentally realistic parameters: 3 diffusion directions, 7 shots for $b=0,17$ shots for $b=550 \mathrm{~s} \mathrm{mm^{-2 }}, 19$ spokes per shot, and 30 coils with a sinusoidal coil model. After simulated radial data sampling [55], white Gaussian noise was added to the raw data to simulate noisy data acquisition. The amplitude of the noise was chosen to match a predefined $S N R=\frac{\|D\|_{2}^{2}}{\|N\|_{2}^{2}}$ of 1.5 for each individual imaging channel, with the raw data $D$ and the noise $N$. The ADC was measured in a region of interest of the numerical phantom as a function of simulated SNR after single-shot reconstruction and multi-shot reconstruction using variants $2 \mathrm{a}, 2 \mathrm{~b}$, and $2 \mathrm{c}$ for processing of the phase maps, see subsection 5.2 .2 . 


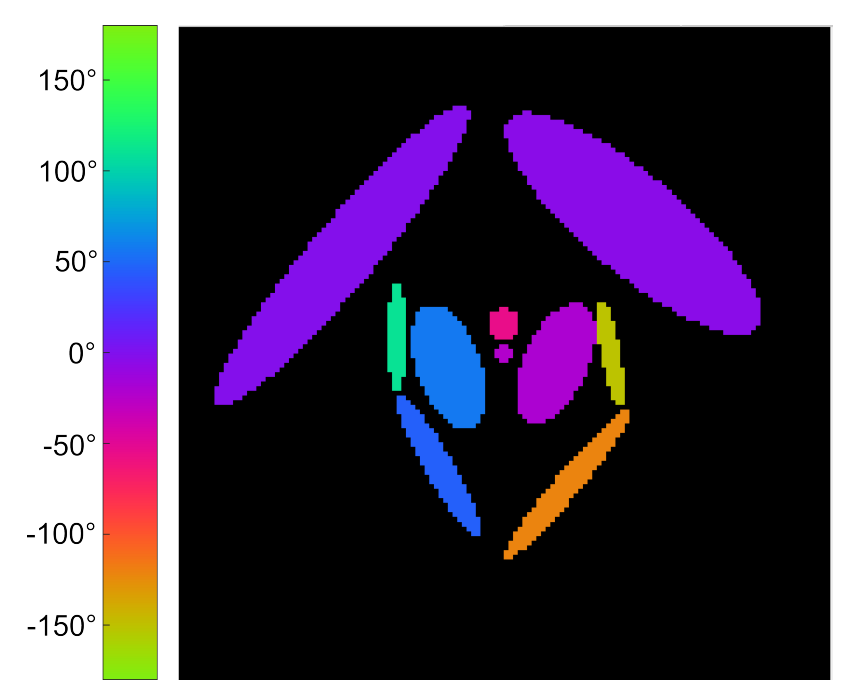

(a) Geometry of the numerical phantom with a random phase for each ellipse.

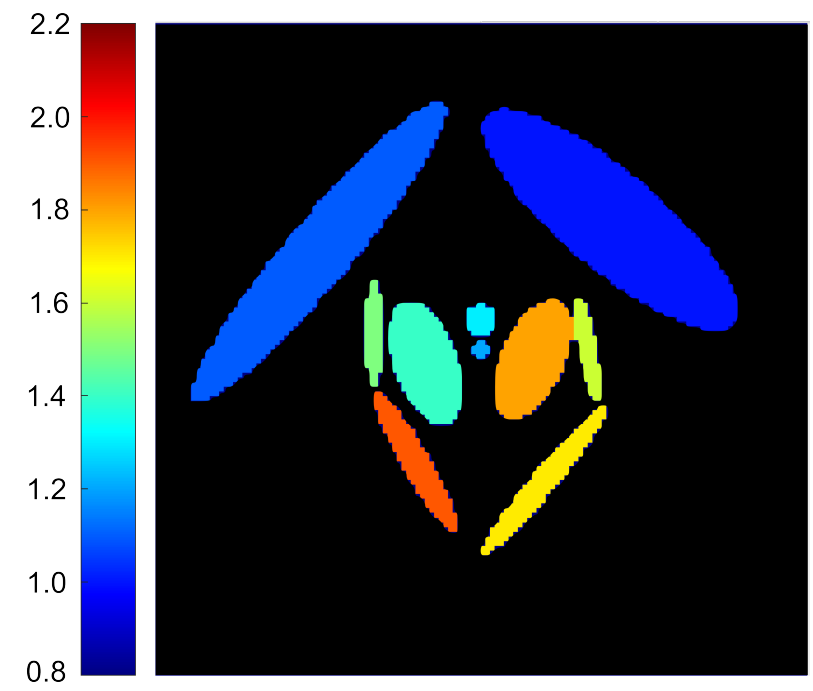

(b) ADC values of the ellipses of the numerical phantom, in units of $10^{-3} \mathrm{~mm}^{-2} \mathrm{~s}^{-1}$

Figure 38 - The numerical phantom used for validation of the image reconstruction algorithm.

In vivo validation of ADC measurements was performed on a region of interest in the central gland of the prostate using an image acquisition protocol with 35 radial spokes and $b=600 \mathrm{~s} \mathrm{~mm}^{-2}$. To assess the dependence of the resulting ADC values on the SNR, the slice thickness was varied between $3.5 \mathrm{~mm}$ and $6.5 \mathrm{~mm}$. As in the numerical experiment, the results were compared for variants $2 \mathrm{a}, 2 \mathrm{~b}$, and $2 \mathrm{c}$ (subsection 5.2.2).

Besides its effect on ADC measurements, multi-shot imaging offers the possibility of increasing the undersampling factor for the individual shots. This is achieved by rotating the sampling trajectory by an angle of $2 \pi /(p * M)$ for each shot, with $\mathrm{p}$ the number of spokes per shot and $\mathrm{M}$ the number of shots.

\subsubsection{Optimization of imaging parameters}

To develop a protocol for in-vivo imaging, adaptation of the following imaging parameters was performed by experimental in-vivo optimization of image quality:

- b-values: Results from a protocol with $b=50 / 400 / 800 s \mathrm{~mm}^{-2}$ were compared with a protocol using $b=50 / 600 \mathrm{~s} \mathrm{~mm}^{-2}$ with regard to image quality and resulting ADC values.

- The repetition time TR was optimized with regard to the image quality of a single diffusionweighted image without post-processing. A sampling trajectory of 15 spokes and the number of averages was adjusted for a constant acquisition time of $72 \mathrm{~s}$ for one diffusion-weighting. The use of TR values similar to, or even smaller than T1 required a series of pre-scans before the start of the sequence to ensure that the signal losses due to T1 decay affect the quality of all sections equally. 
- At a fixed TR chosen in the previous step, the effect of the number of spokes on the traceweighted images at $b=600 \mathrm{~s} \mathrm{~mm}^{-2}$ was investigated.

\subsubsection{Comparison with EPI}

The diffusion-weighted STEAM MRI protocol with optimized imaging parameters was applied to a healthy volunteer. The resulting trace-weighted images and ADC maps were qualitatively compared against the results from the commercial clinical EPI protocol. The only modifications to this protocol were an adjustment of the b-values and repetition times to the STEAM protocol to achieve a fair comparison. Reconstruction and further processing of the EPI results uses the default GRAPPA algorithm and settings provided by the vendor for use in clinical examinations. Both protocols are shown in table 3 .

\begin{tabular}{|c|c|c|}
\hline Sequence & STEAM & EPI \\
\hline In-plane resolution $[\mathrm{mm}]$ & 1.43 & 1.43 \\
\hline Matrix size & 140 & 140 \\
\hline Field of view [mm] & 200 & 200 \\
\hline Slice thickness [mm] & 3.5 & 3.5 \\
\hline Number of slices & 21 & 21 \\
\hline b-value $\# 1\left[\begin{array}{lll}s & \left.\mathrm{~mm}^{-2}\right]\end{array}\right.$ & 50 & 50 \\
\hline Averages/shots \#1 & 7 & 7 \\
\hline b-value $\# 2\left[s m^{-2}\right]$ & 600 & 600 \\
\hline Averages/shots \#2 & 17 & 17 \\
\hline Diffusion directions & 3 & 3 \\
\hline $\mathrm{TR}[\mathrm{ms}]$ & 5000 & 5000 \\
\hline Total acquisition time & $6 \min 25 \mathrm{~s}$ & $6 \min 25 \mathrm{~s}$ \\
\hline Bandwidth [Hz/Pixel] & 200 & 1700 \\
\hline Radial spokes & 19 & - \\
\hline TSE [ms] & 30.8 & 61 \\
\hline $\mathrm{TR} \alpha[\mathrm{ms}]$ & 7.72 & - \\
\hline $\mathrm{TE}[\mathrm{ms}]$ & 8.92 & - \\
\hline
\end{tabular}

Table 3 - Optimized imaging protocols used for final comparisons of image quality

\subsection{Results}

\subsubsection{Image reconstruction: numerical phantom}

Phase maps reconstructed from the numerical phantom with a raw data SNR of 1.5 per channel are shown in figure 39. The phase map reconstructed without further processing is strongly corrupted by noise. Both processing methods for phase maps, k-space truncation and NLM, yield phase maps of superior quality; the result for NLM is slightly superior to k-space truncation with regard 


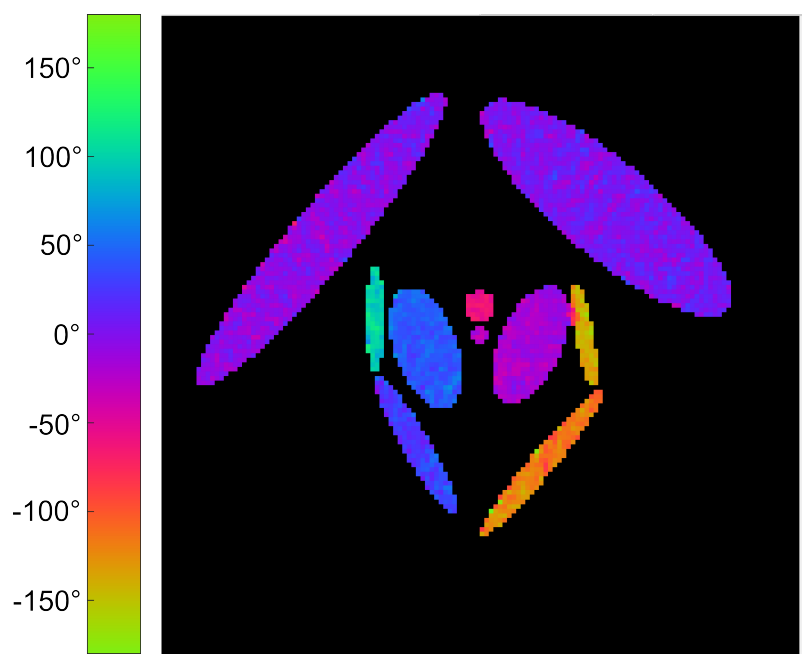

(a) Phase maps without further processing

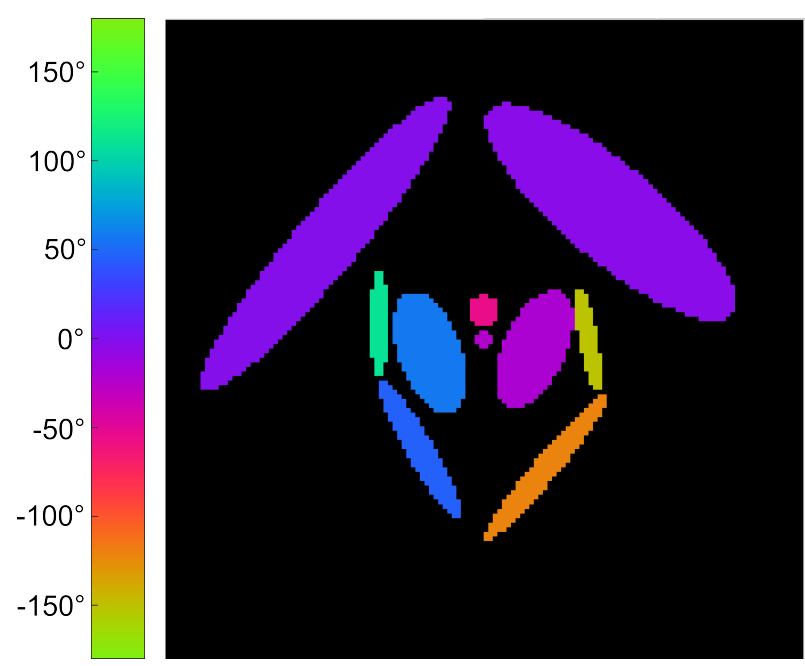

(c) True phase map for the simulated data

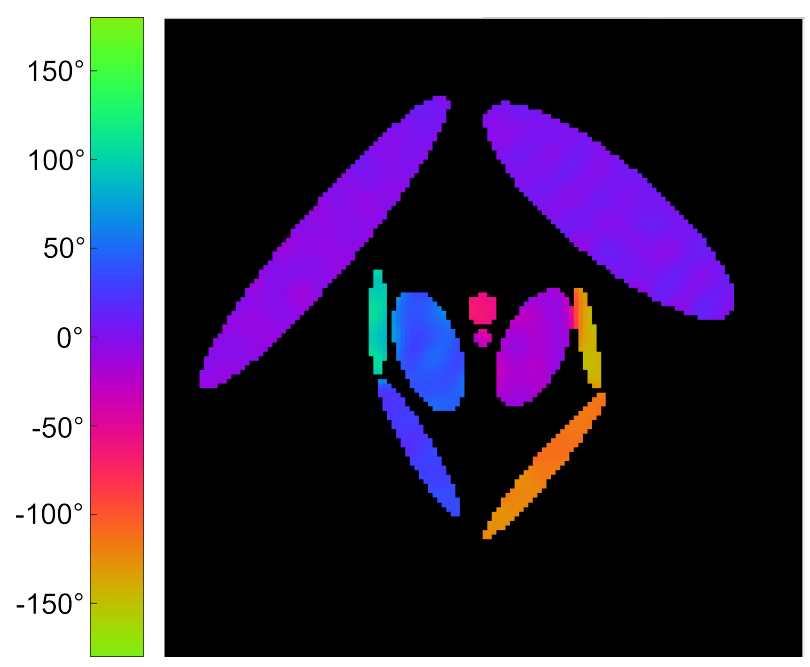

(b) Phase map reconstructed from truncated kspace

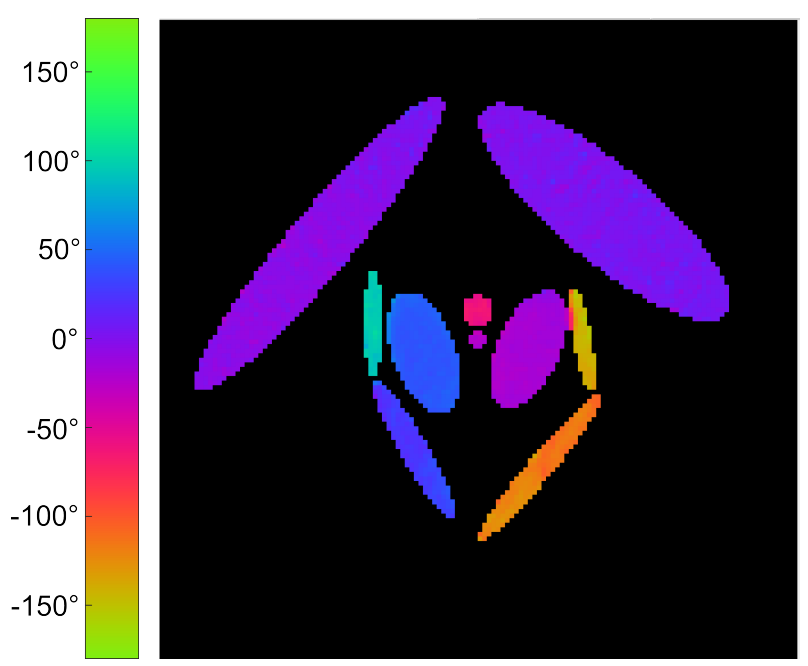

(d) Phase map denoised with non-local means

Figure 39 - Reconstructed phase maps of numerical phantom the SNR per receive channel in the raw data was 1.5 .

to the suppression of low frequency noise and spatial acuity near boundaries between two regions of constant phase.

The effect of the different phase maps on the reconstructed ADC maps is seen in figure 40 . Multi-shot reconstruction using the unprocessed, noisy phase maps leads to a systematic underestimation of the ADC. With either of the two processing methods for the phase maps, the resulting ADC values correspond to the ground truth, and the image qualities of the resulting ADC maps are similar to each other.

ADC evaluation in two regions of interest after single-shot reconstruction and multi-shot reconstruction with and without phase map processing shows a dependence of the minimal SNR per channel for accurate ADC measurement on the reconstruction method (figure 41). For single- 
and multi-shot reconstruction without phase map processing, this value was 3, for multi-shot reconstruction with phase map processing using NLM or k-space truncation, it was 1.5.

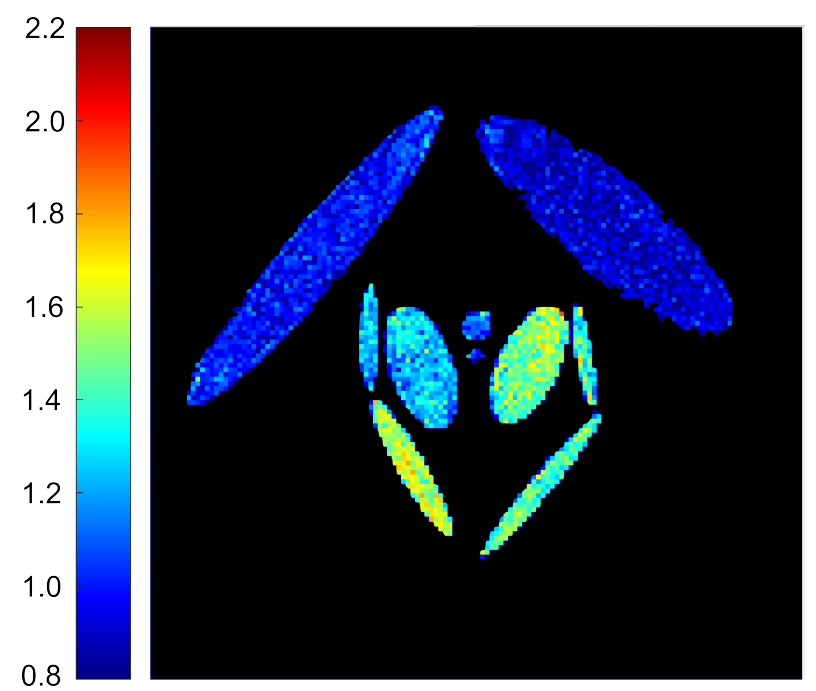

(a) Multi-shot reconstruction, phase maps not processed further

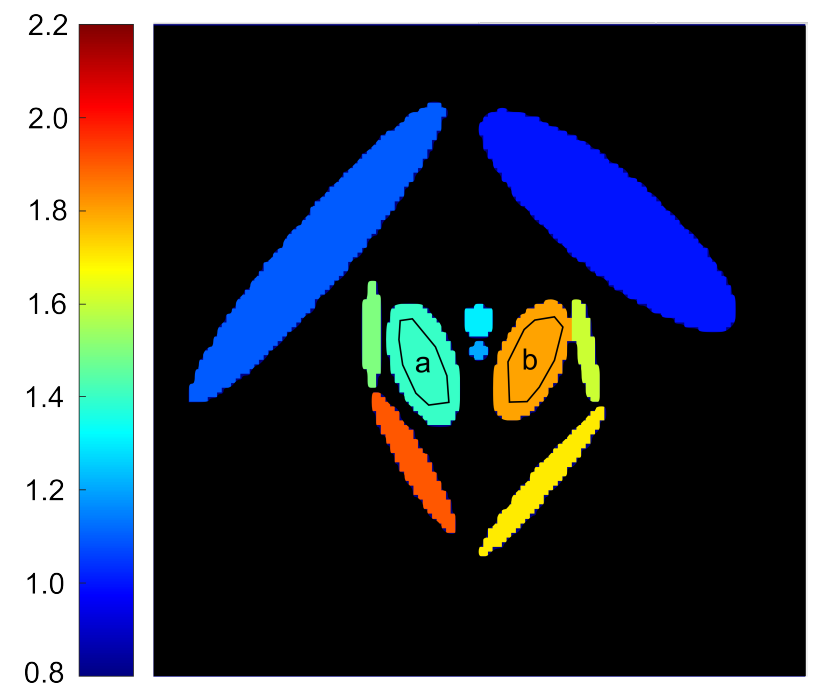

(c) True ADC values in the simulated data. Regions a and $b$ used for quantitative evaluation (see figure 41)

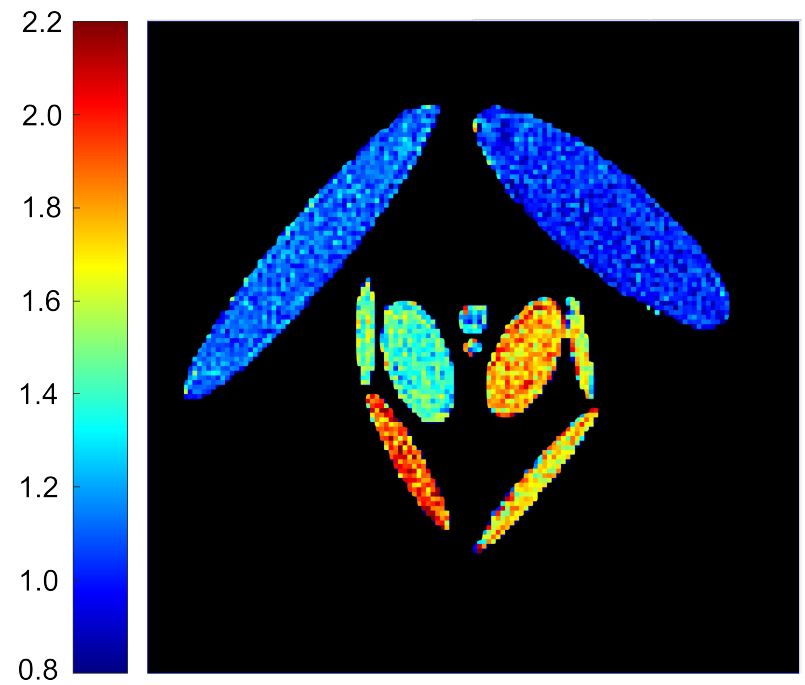

(b) Multi-shot reconstruction, phase maps from truncated k-space

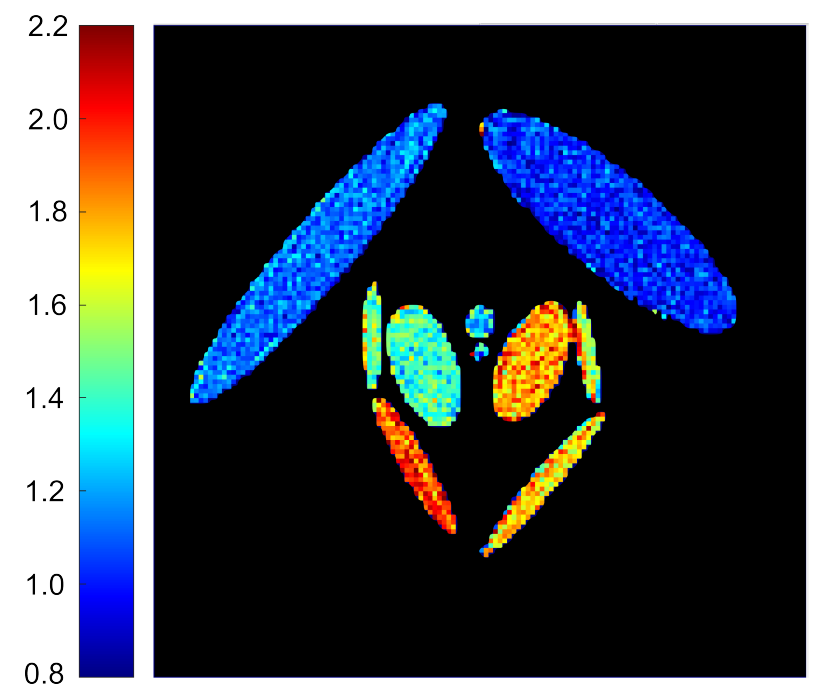

(d) Multi-shot reconstruction, phase maps denoised with non-local means

Figure 40 - Multi-shot reconstruction of numerical phantom, ADC maps. The ADC is given in units of $10^{-3} \mathrm{~mm}^{-2} \mathrm{~s}^{-1}$. 


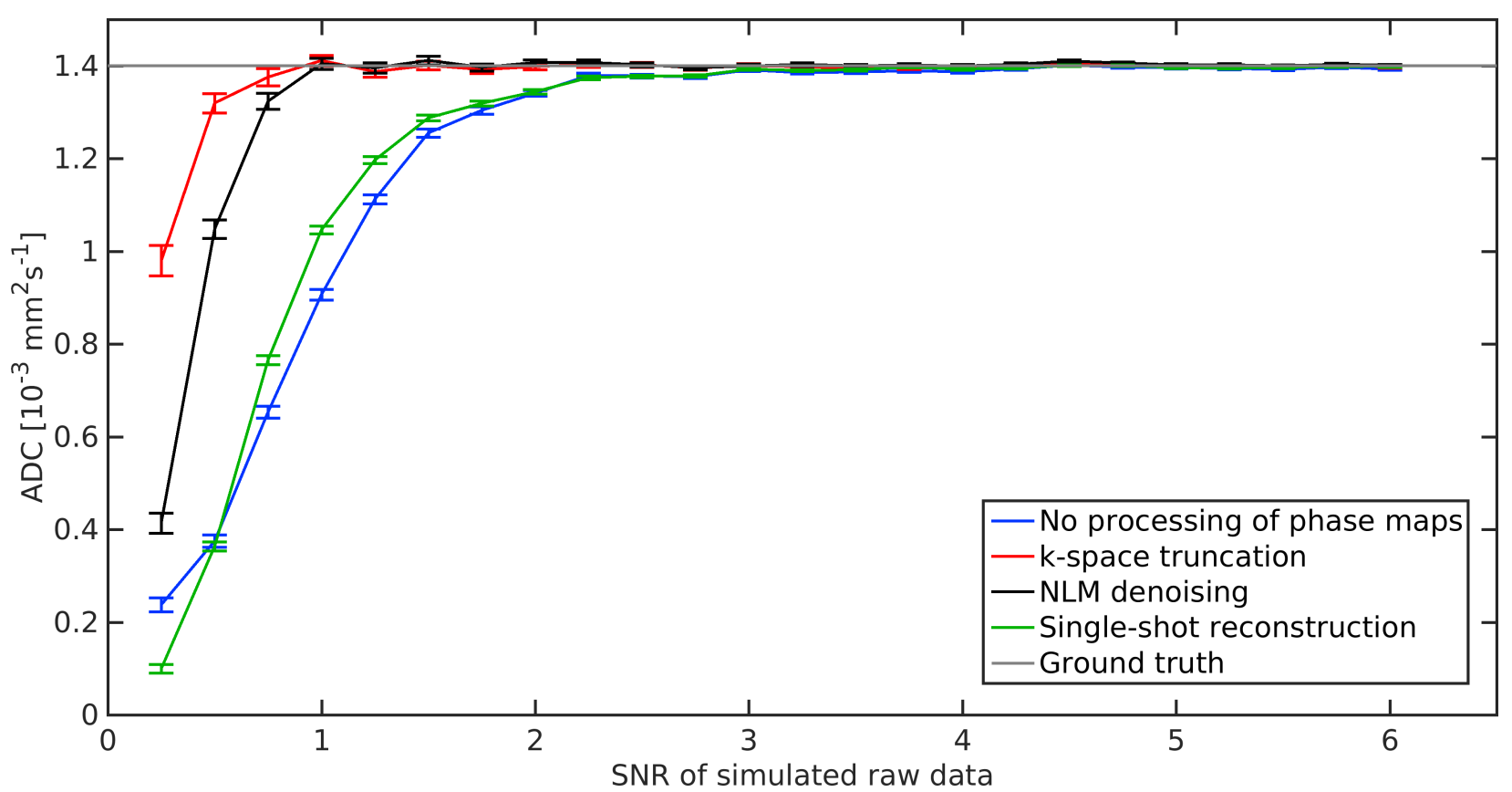

(a) Reconstructed ADC values in region a

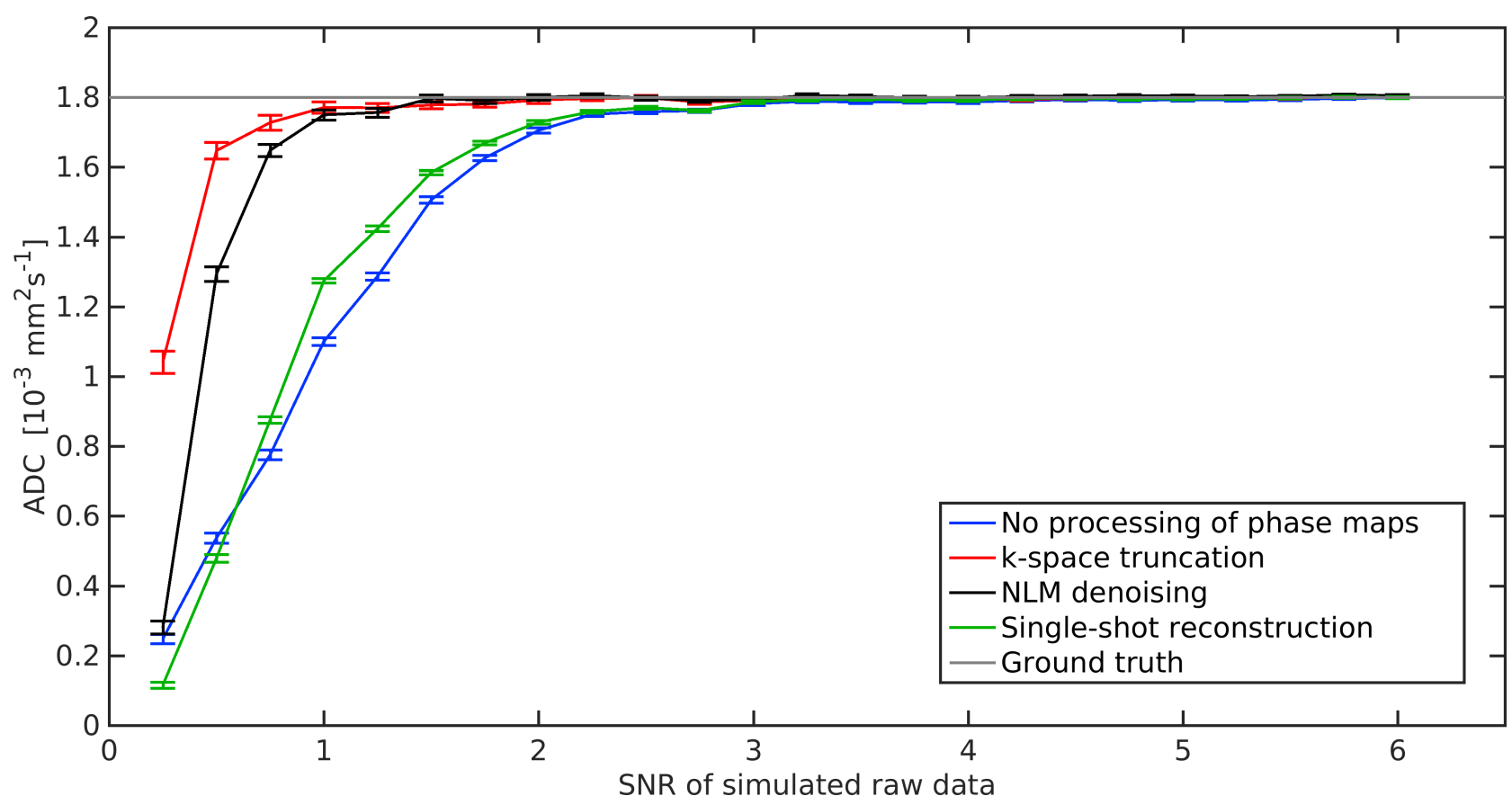

(b) Reconstructed ADC values in region b

Figure 41 - ADC values measured from reconstructions of the numerical phantom. The regions for evaluation are shown in figure 40 .

\subsubsection{Image reconstruction: In vivo imaging of the prostate}

The phase maps acquired with a slice thickness of $3.5 \mathrm{~mm}$ have a patchy appearance with a large degree of low-frequency noise after reconstruction from a truncated k-space (figure 42c). The phase map calculated with NLM denoising shows some remaining high-frequency noise (figure 42b), but 
no artificial patches. The unprocessed phase map shown in figure $42 \mathrm{a}$ is clearly dominated by noise.

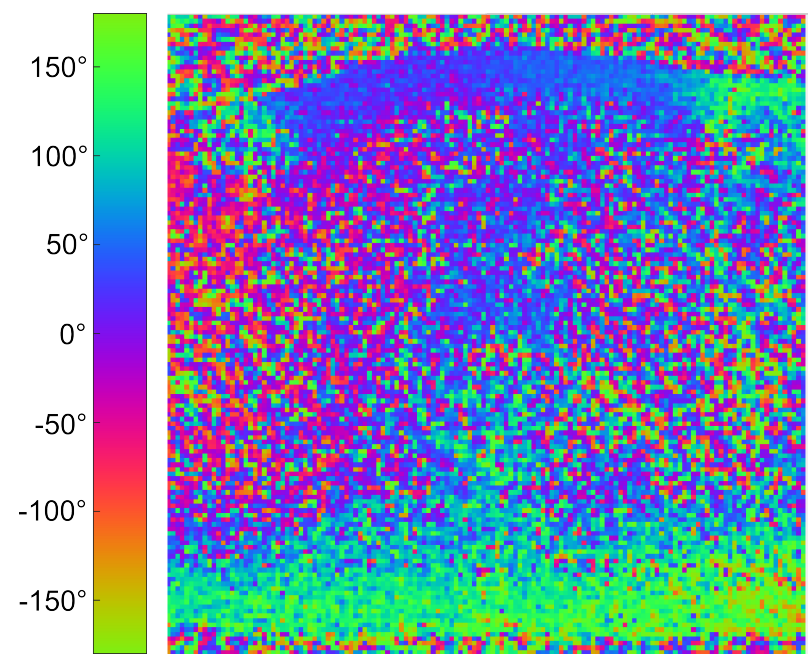

(a) Phase map without processing

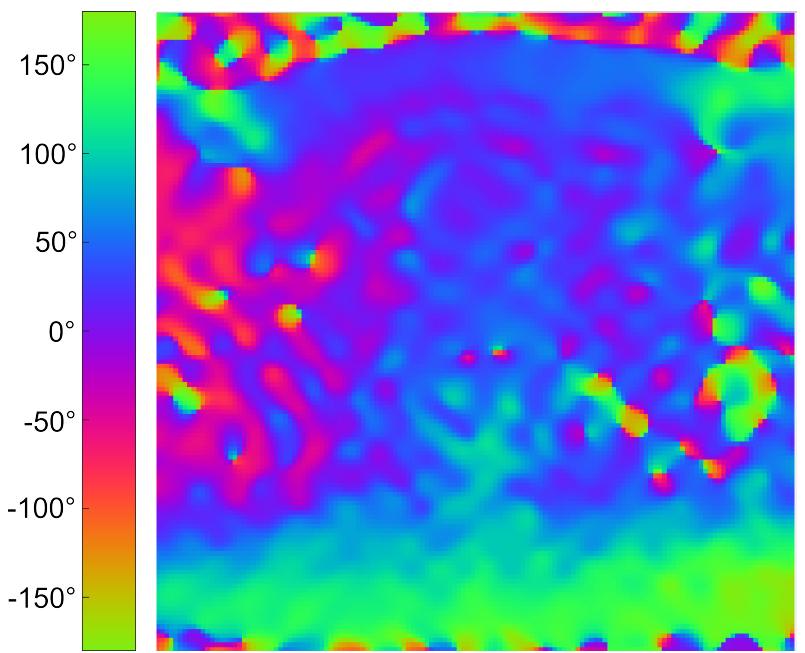

(c) Phase map truncated in k-space (factor 0.2)

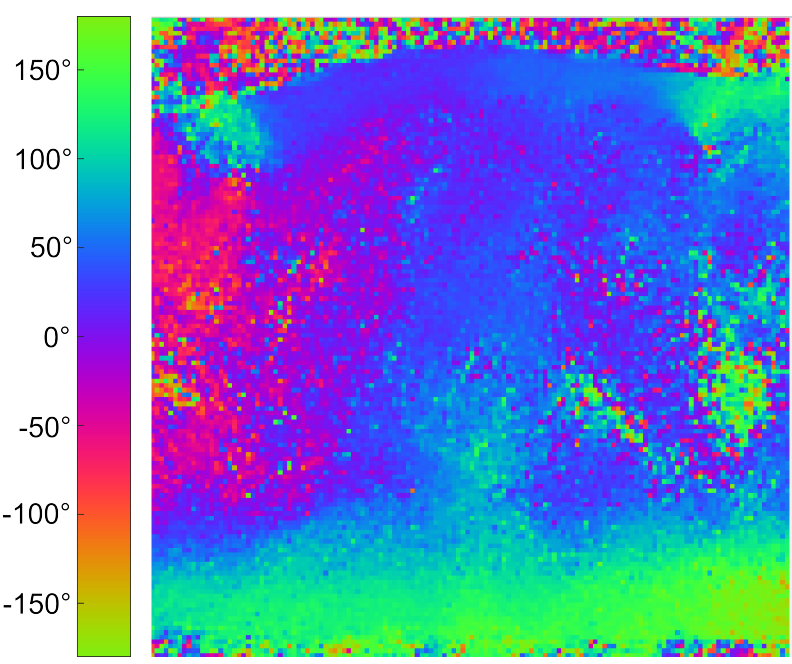

(b) Phase map denoised with non-local means

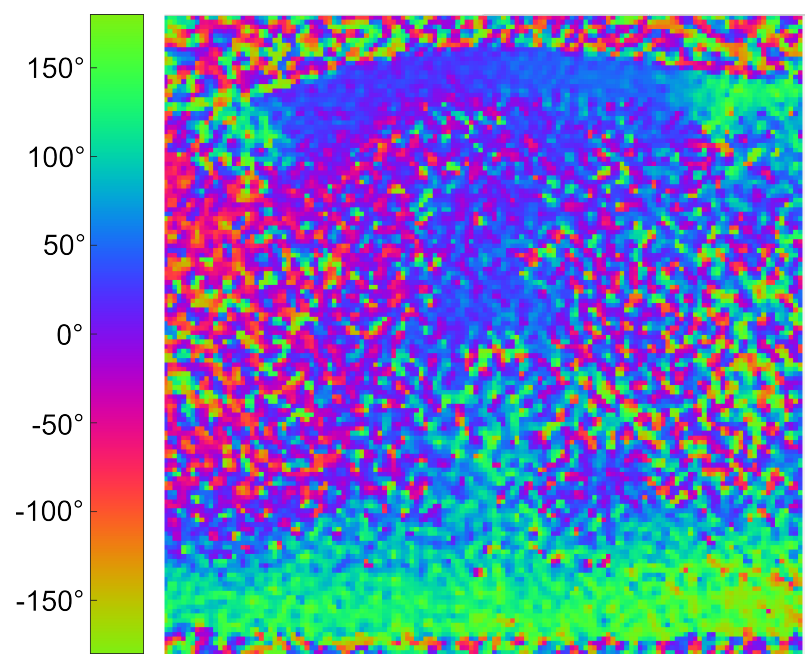

(d) Phase map truncated in k-space (factor 0.6)

Figure 42 - Phase maps reconstructed in vivo, slice thickness $3.5 \mathrm{~mm}$.

ADC evaluation in the central gland on in-vivo acquisitions of the prostate shows a strong dependence of the ADC values on the slice thickness for single- or multi-shot reconstruction without phase map processing, whereas phase map processing yields ADC values independent from the slice thickness (figures 43, 44) and in agreement with the reference value of $(1.47 \pm 0.24) * 10^{-3} \mathrm{~mm}^{2} \mathrm{~s}^{-1}$ $[12$.

The two different phase map processing methods, NLM denoising and k-space truncation, yield very similar image quality in the trace-weighted image and the ADC map (see figure 45). The differences of the ADC values in the central gland are not significant (figure 44). 


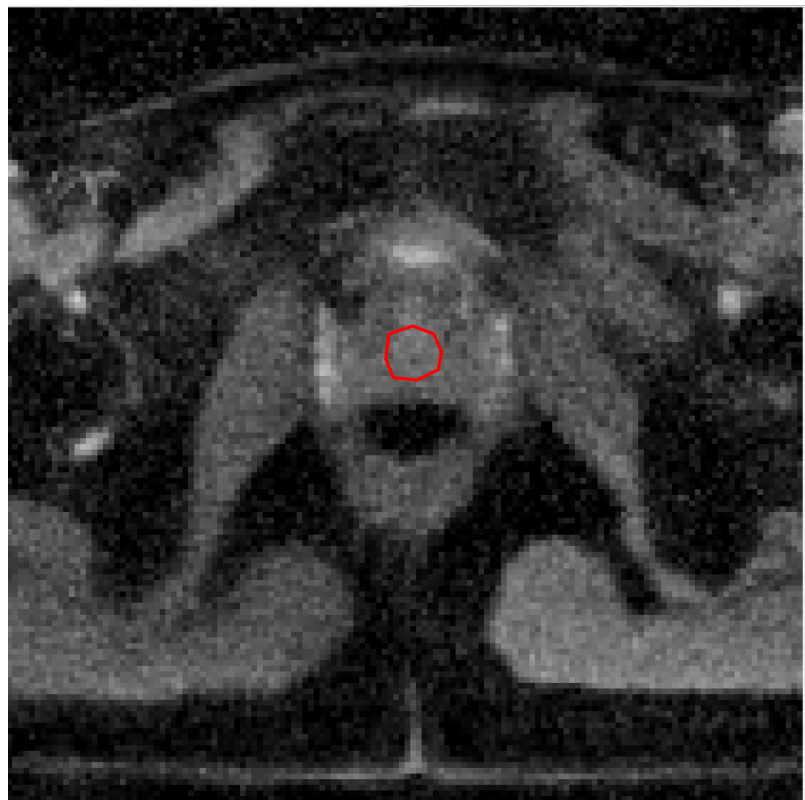

(a) ADC map, no processing of phase maps, slice thickness $3.5 \mathrm{~mm}$

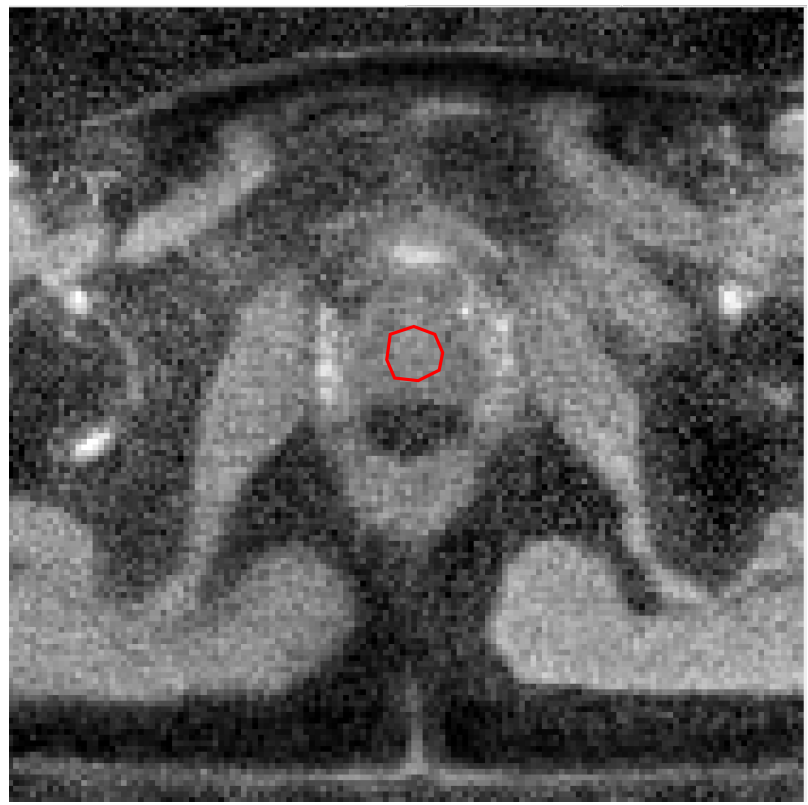

(c) ADC map, denoising with non-local means, slice thickness $3.5 \mathrm{~mm}$

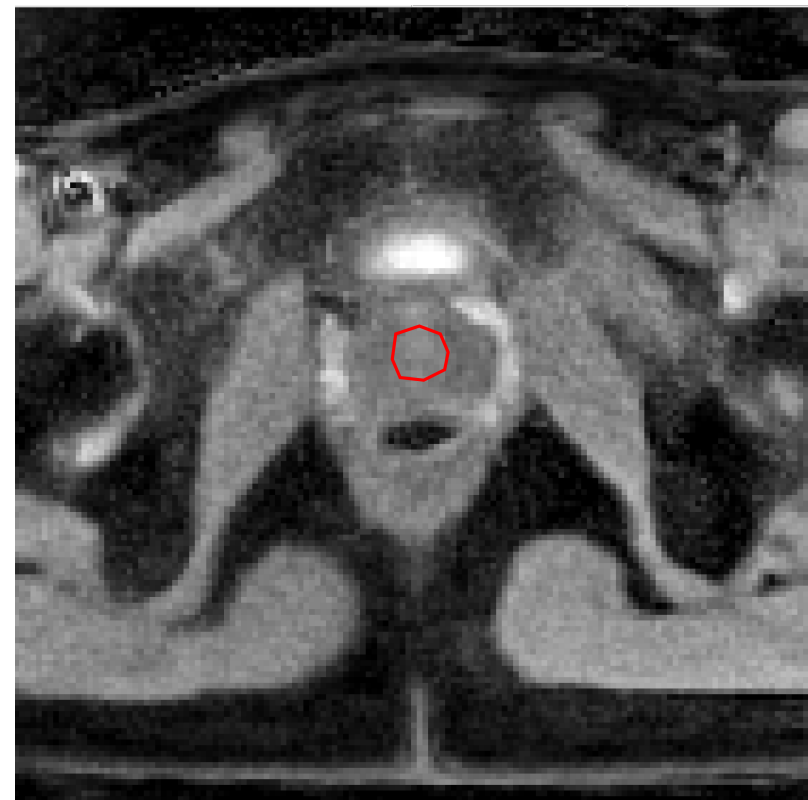

(b) ADC map, no processing of phase maps, slice thickness $5.5 \mathrm{~mm}$

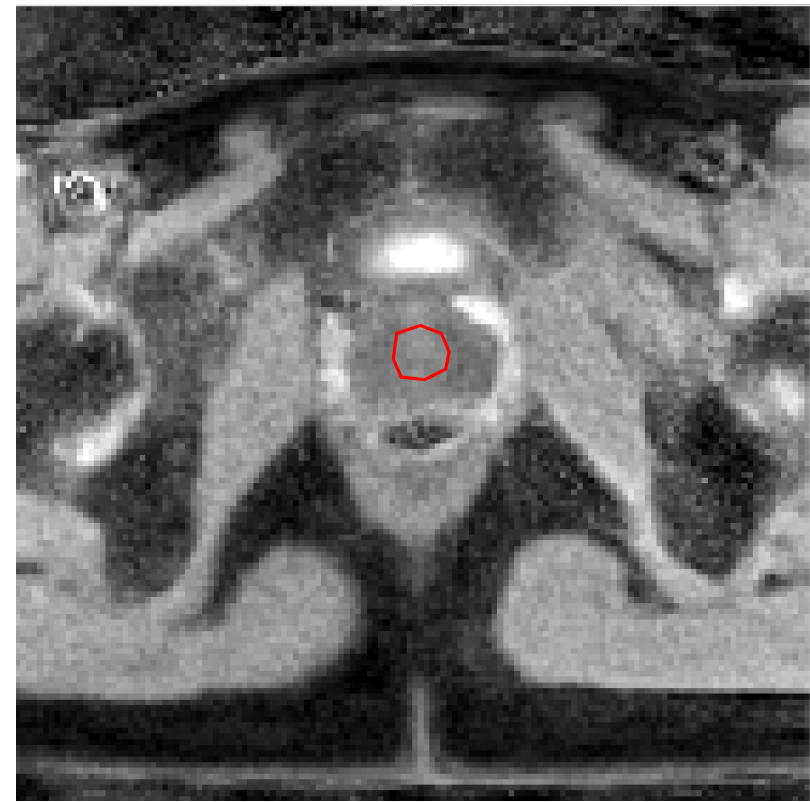

(d) ADC map, denoised with non-local means, slice thickness $5.5 \mathrm{~mm}$

Figure 43 - ADC maps of the prostate computed with and without denoising of phase maps. The region of interest delineated in red was used for quantitative evaluation (see figure 44). 


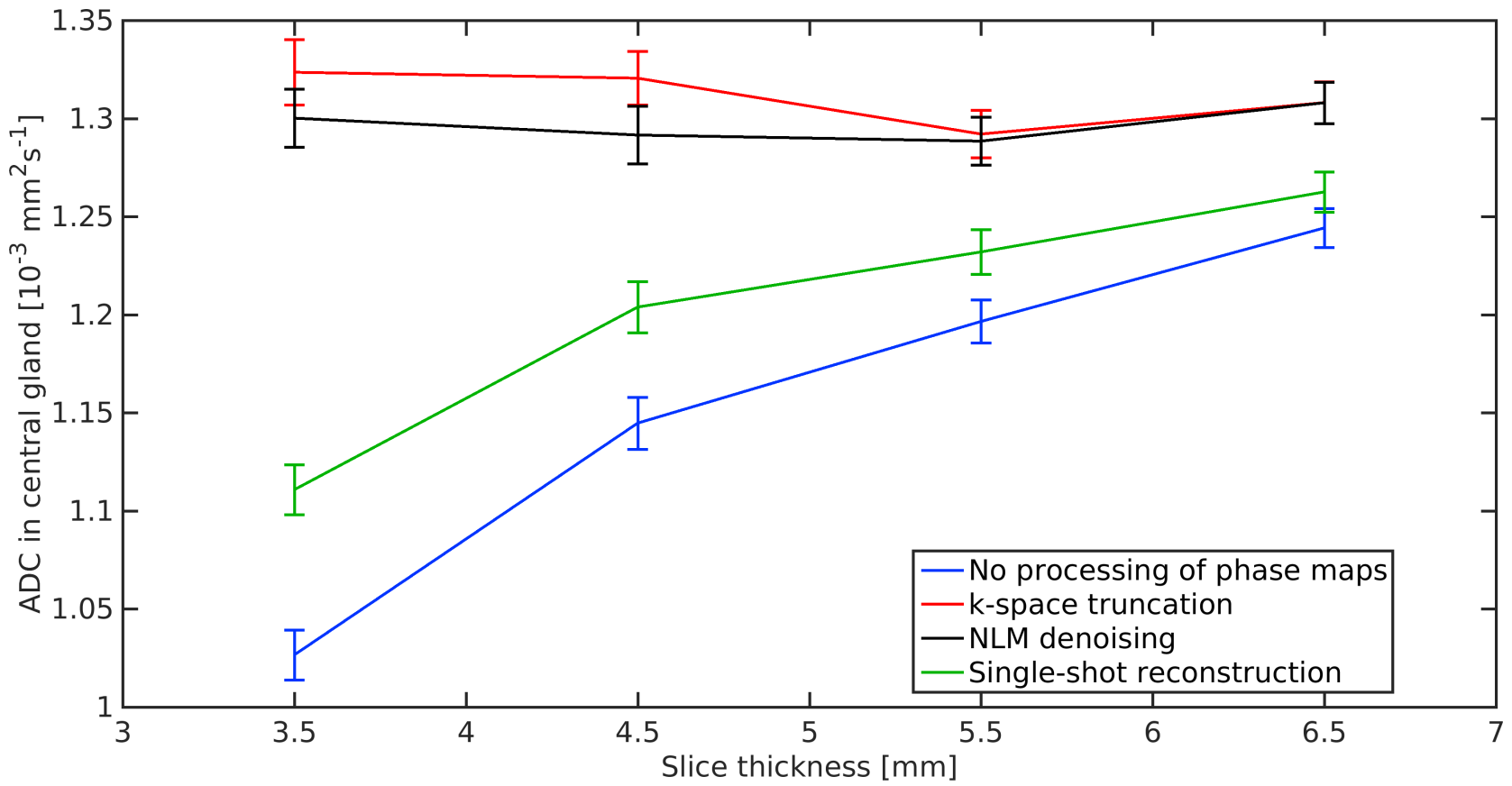

Figure 44 - Dependence of measured ADC values on the slice thickness for single shot reconstruction (see chapter 4), multi-shot reconstruction without processing of phase maps [61], and multi-shot reconstruction with additional processing of phase maps. The ADC (mean \pm standard error) was evaluated on the region in the central gland shown in figure 43 . 


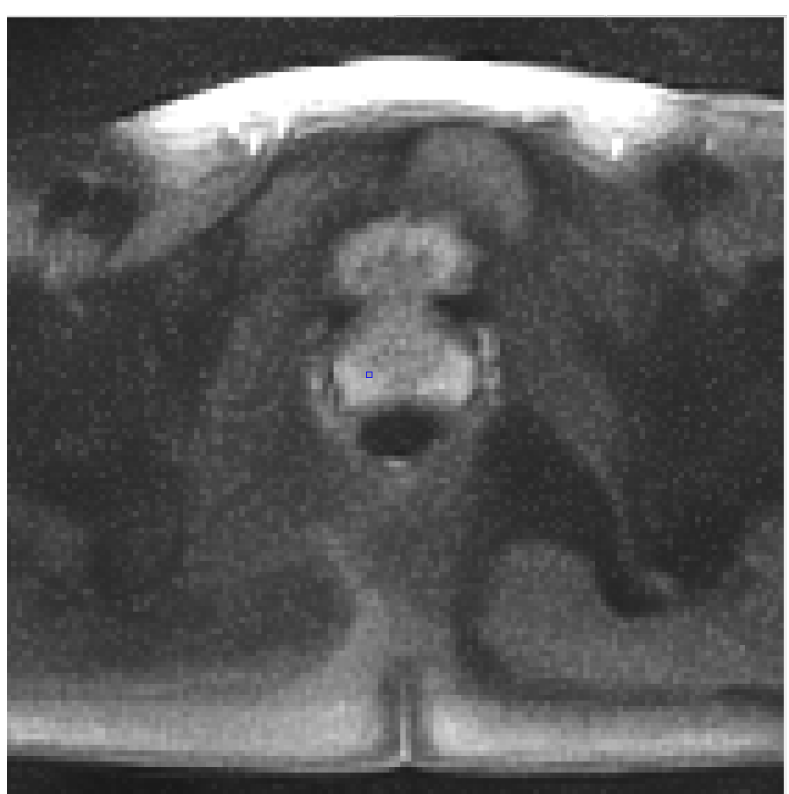

(a) Trace weighted image, k-space truncation

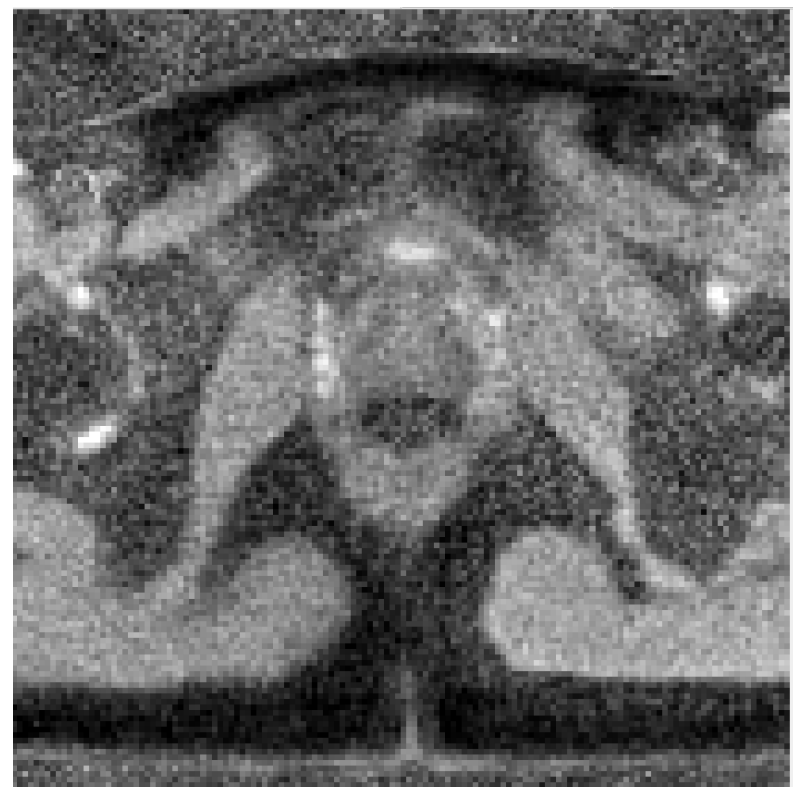

(c) ADC, k-space truncation

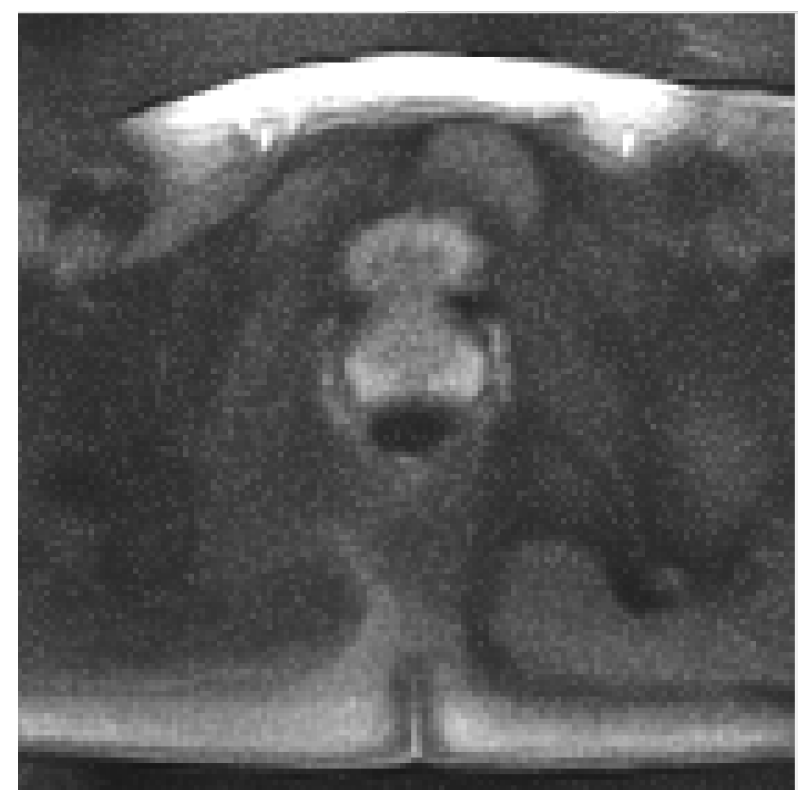

(b) Trace-weighted image, NLM denoising

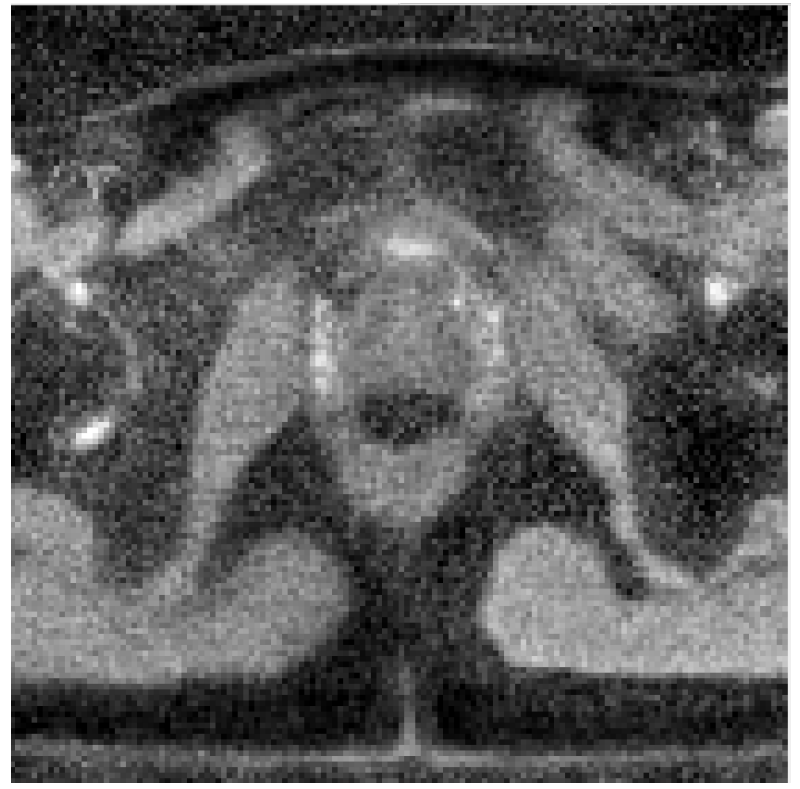

(d) ADC, NLM denoising

Figure 45 - Comparison of resulting image quality with reconstruction using k-space truncation and NLM denoising of phase maps 


\subsubsection{Parameter optimization for multi-shot prostate imaging}

b-values In figure 46, the image contrast of trace-weighted prostate images and the ADC maps is shown as a function of the b-value. The trace-weighted image with $b=800 \mathrm{~s} \mathrm{~mm}^{-2}$ shows inferior image quality which can be attributed to low SNR. Two ADC maps were calculated, one using data with $b=50 / 400 / 800 \mathrm{~s} \mathrm{~mm}^{-2}$ and one from data with $b=50 / 600 \mathrm{~s} \mathrm{~mm}^{-2}$. In the central gland, the ADC measured with $b=50 / 400 / 800 s \mathrm{~mm}^{-2}$ was $(1.24 \pm 0.02) * 10^{-3} \mathrm{~mm}^{2} \mathrm{~s}^{-1}$, i.e. significantly lower than the value of $(1.30 \pm 0.02) * 10^{-3} \mathrm{~mm}^{2} \mathrm{~s}^{-1}$ measured with $b=50 / 600 \mathrm{~s} \mathrm{~mm}^{-2}$. The global mean ADC for the entire slice was $12.2 \%$ lower for $b=50 / 400 / 800 \mathrm{~s} \mathrm{~mm}^{-2}$.

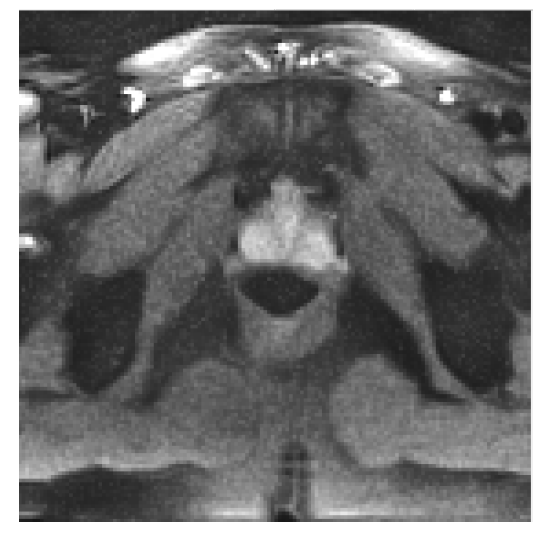

(a) Trace weighted image, b50

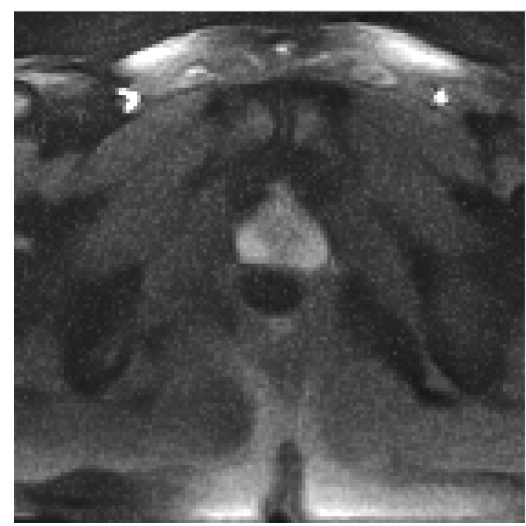

(d) Trace weighted image, b400

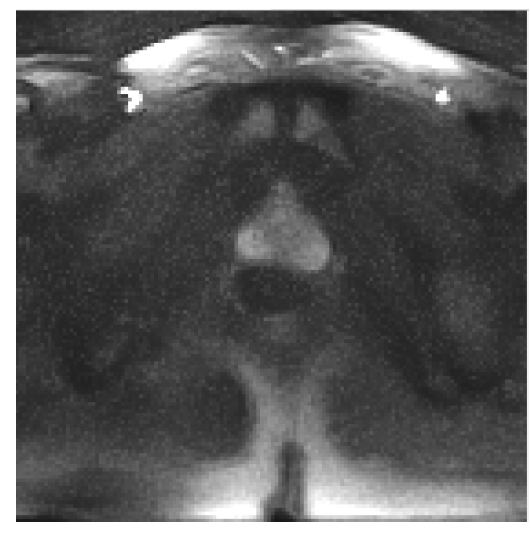

(b) Trace weighted image, b600

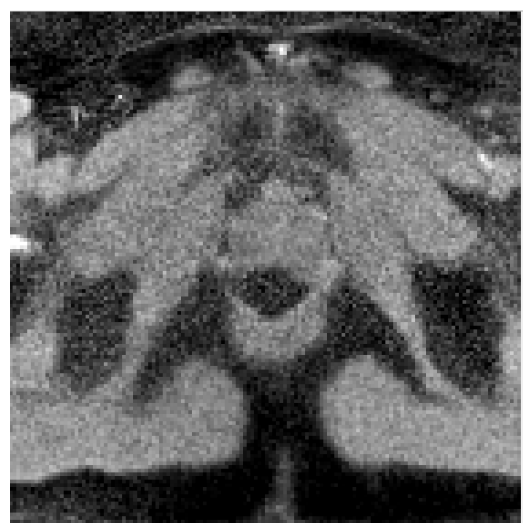

(e) $\mathrm{ADC}, \mathrm{b} 50 / 600$

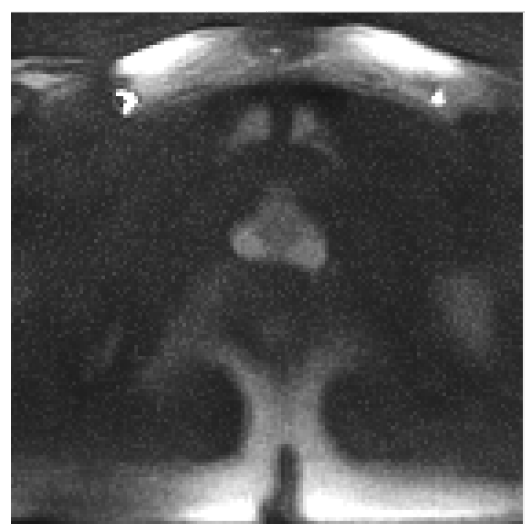

(c) Trace weighted image, b800

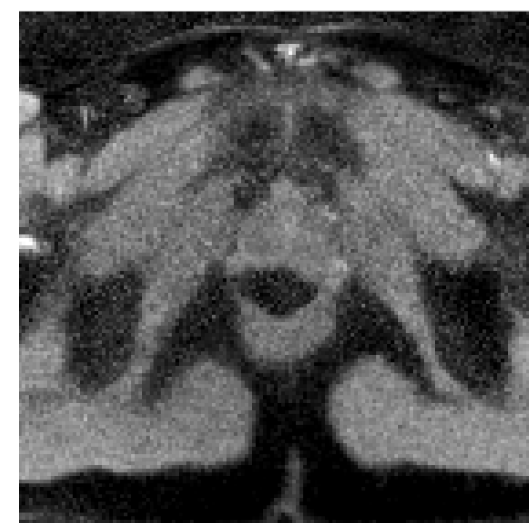

(f) $\mathrm{ADC}, \mathrm{b} 50 / 400 / 800$

Figure 46 - Contrast and image quality as a function of the b-value. All b-values are given in units of $s \mathrm{~mm}^{-2}$.

Repetition time Figure 47 shows that a rotated k-space trajectory for multi-shot imaging allows for 15 spokes per shot and 7 shots without undersampling artifacts, whereas 15 spokes lead to undersampling artifacts if the $\mathrm{k}$-space trajectory is not rotated. The possibility for undersampling creates a greater flexibility for the choice of the repetition time TR, which can be experimentally 
optimized with respect to image quality. Figure 48 shows that a single diffusion-weighted image at $b=600 \mathrm{~s} \mathrm{mm^{-2 }}$ is least corrupted by noise with a repetition time of $4.8 \mathrm{~s}$ or $5.5 \mathrm{~s}$, whereas higher or lower repetition times lead more noisy images if the total acquisition time is kept constant. Therefore, a repetition time of $5 \mathrm{~s}$ is a suitable choice.

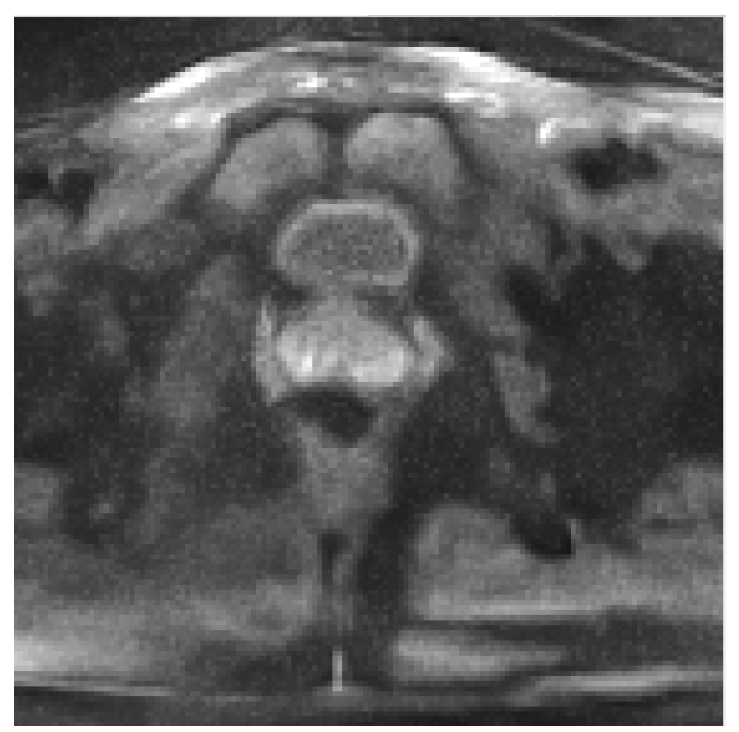

(a) No k-space rotation

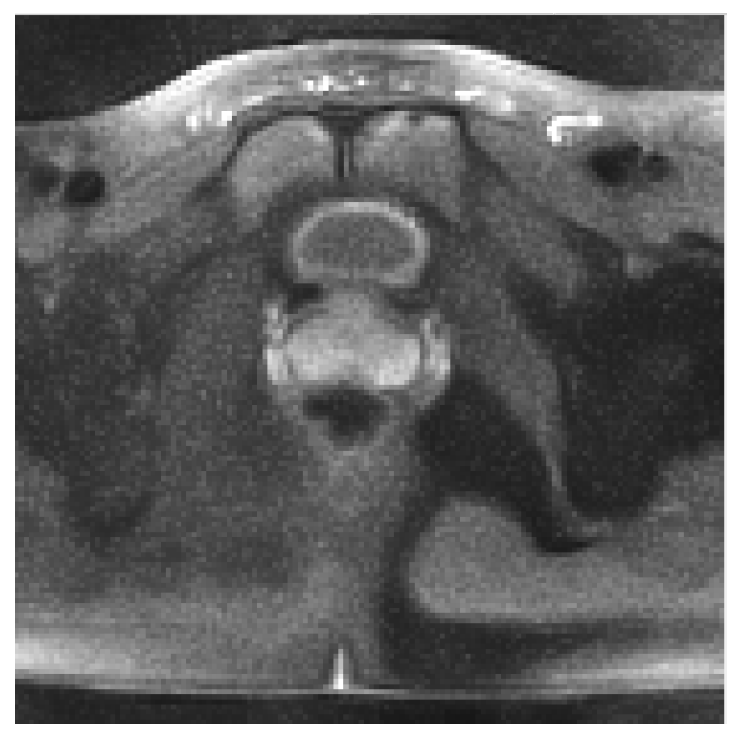

(b) Rotated k-space trajectory

Figure 47 - Effect of rotated k-space trajectory on trace-weighted images from undersampled data (15 spokes). The images were reconstructed with NLM denoising of phase maps.

Number of radial spokes The image quality of trace-weighted images at $b=600 \mathrm{~s} \mathrm{~mm}^{-2}$ was optimized with respect to the number of radial spokes (figure 49). The signal-to-noise ratio increases with a decreasing number of spokes, however, for less than 15 spokes, the images are corrupted by undersampling artifacts. A pragmatic balance between these two effects is achieved for 19 radial spokes. 


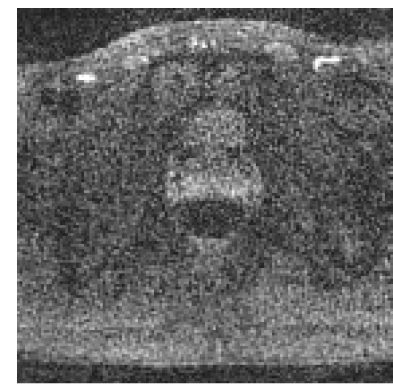

(a) $3 \times \mathrm{TR}=24 \mathrm{~s}$

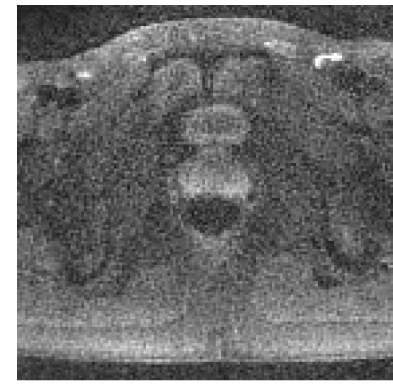

(e) $11 \times \mathrm{TR}=6.5 \mathrm{~s}$

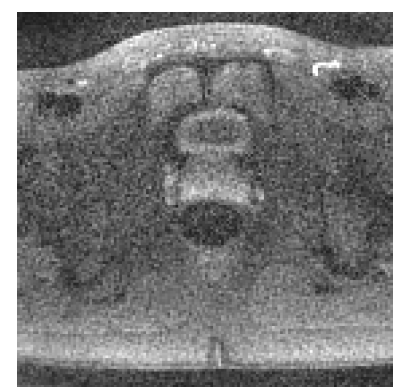

(i) $19 \times \mathrm{TR}=3.8 \mathrm{~s}$

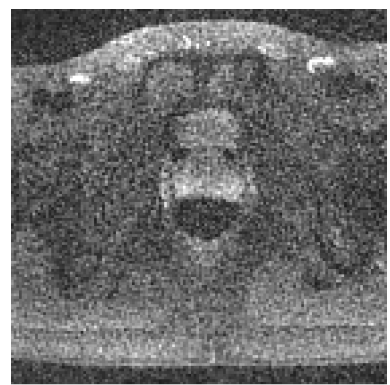

(b) $5 \times \mathrm{TR}=14.4 \mathrm{~s}$

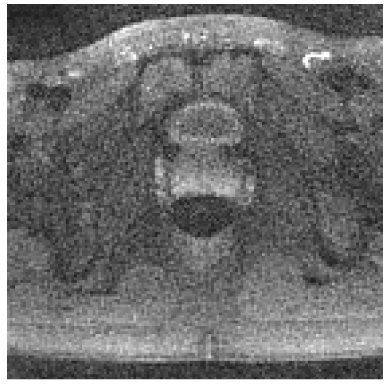

(f) $13 \times \mathrm{TR}=5.5 \mathrm{~s}$

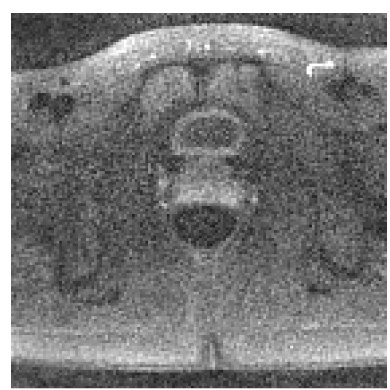

(j) $25 \mathrm{x} \mathrm{TR}=2.9 \mathrm{~s}$

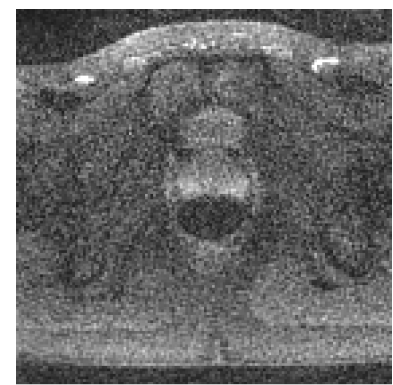

(c) $7 \times \mathrm{TR}=10.3 \mathrm{~s}$

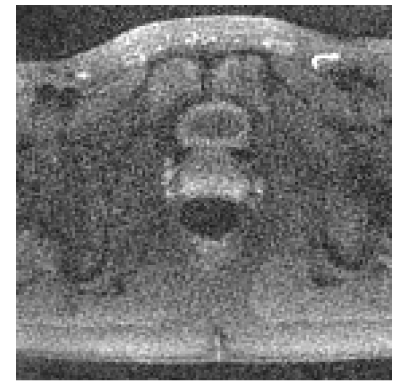

(g) $15 \times \mathrm{TR}=4.8 \mathrm{~s}$

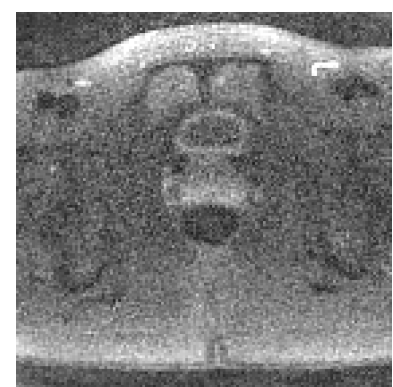

(k) $31 \mathrm{x} \mathrm{TR}=2.3 \mathrm{~s}$

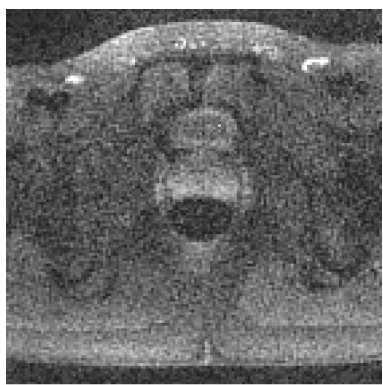

(d) $9 \mathrm{x} \mathrm{TR}=8 \mathrm{~s}$

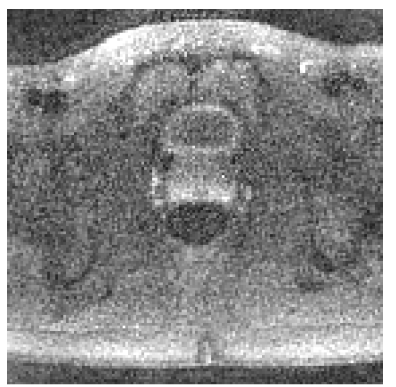

(h) $17 \times \mathrm{TR}=4.2 \mathrm{~s}$

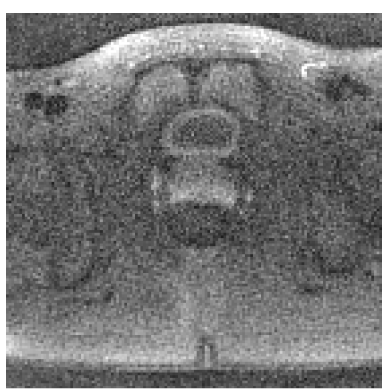

(1) $35 \times \mathrm{TR}=2.1 \mathrm{~s}$

Figure 48 - Experimental optimization of repetition time choice, with a constant acquisition time of $72 \mathrm{~s}$, single diffusion-weighting of b600, 15 spokes. The sub-captions indicate the numbers of shots and the repetition times. 


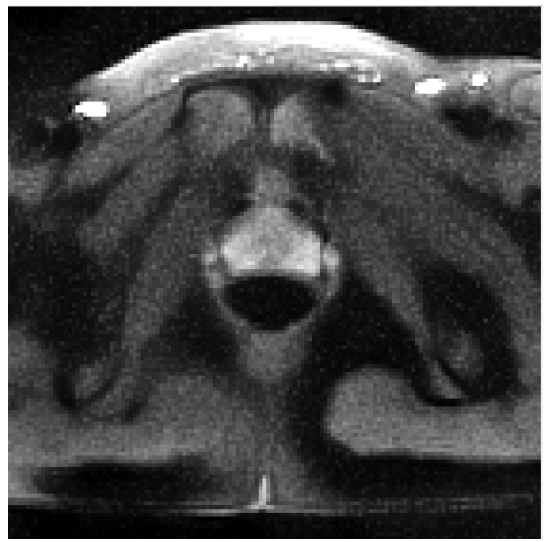

(a) 35 spokes

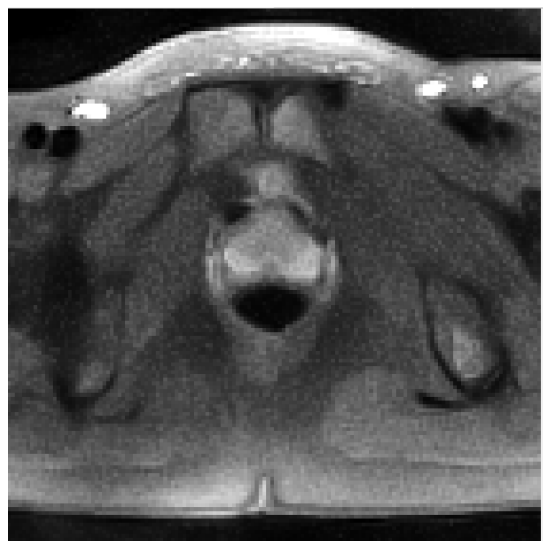

(d) 15 spokes

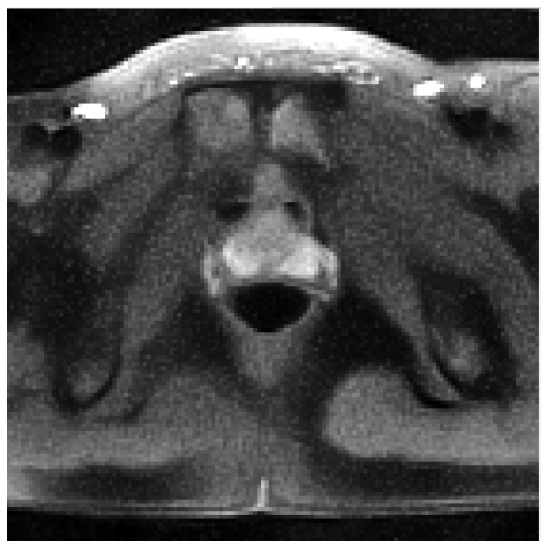

(b) 27 spokes

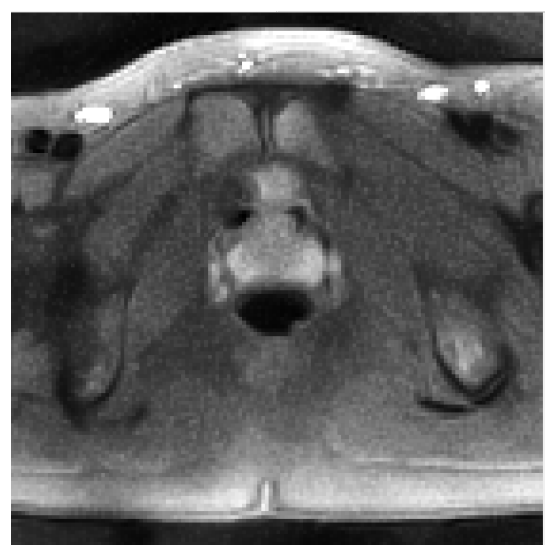

(e) 11 spokes

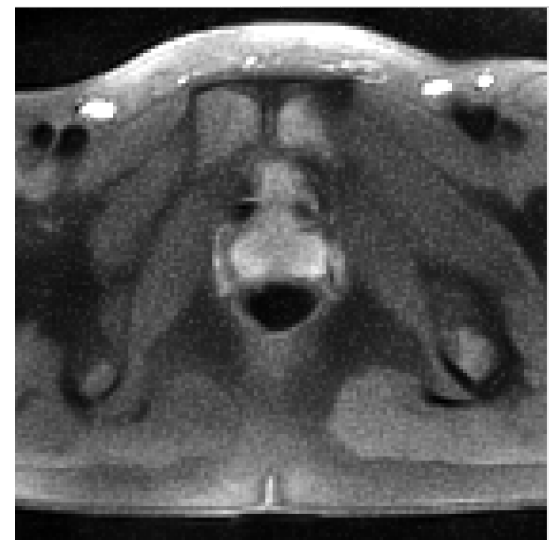

(c) 19 spokes

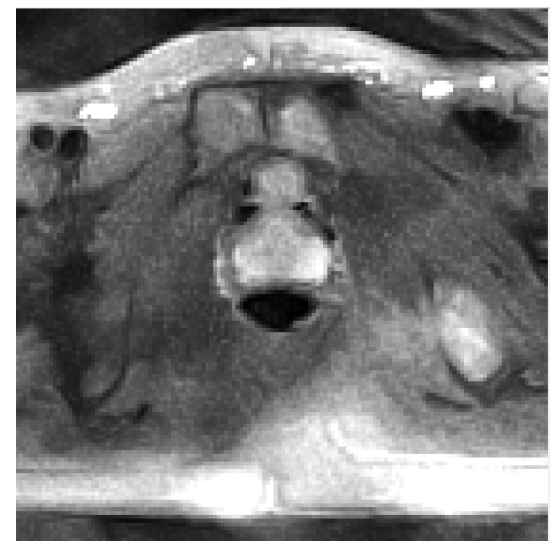

(f) 7 spokes

Figure 49 - Quality of Trace-weighted images as a function of the number of radial spokes 


\subsubsection{Comparison with EPI}

The trace-weighted images and ADC maps acquired with the optimized STEAM protocol and the adapted commercial EPI protocol (table 3) are shown in figure 50. The image quality provided by the STEAM protocol shows a potential for the acquisition of diagnostic information, despite lower image SNR compared to EPI. However, EPI acquisition produces severe distortions at the boundary between the prostate and the colon in both the trace-weighted images and the ADC maps. Here, the STEAM protocol yields superior spatial acuity and a clearly defined and undistorted tissue boundary.

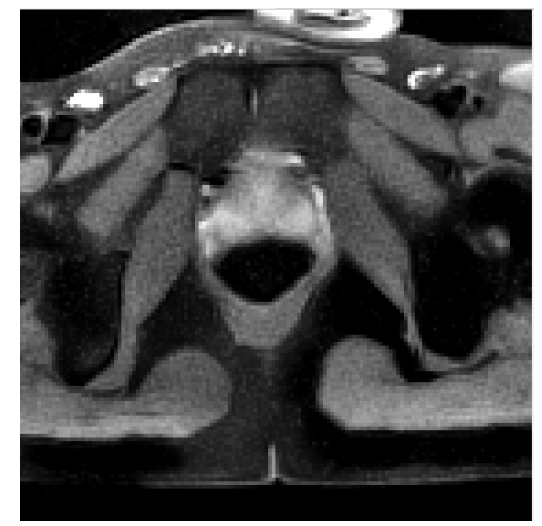

(a) Trace weighted image, b50, STEAM

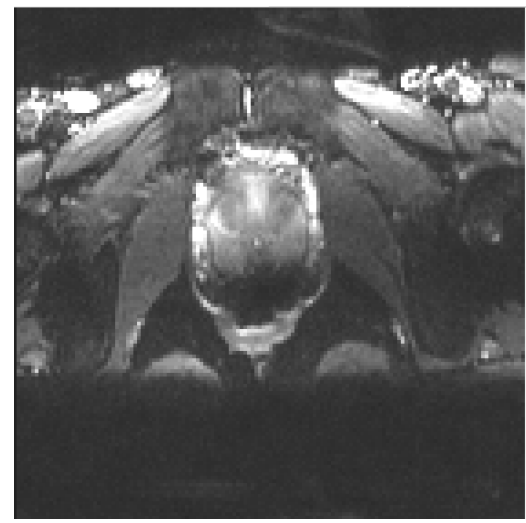

(d) Trace weighted image, b50, EPI

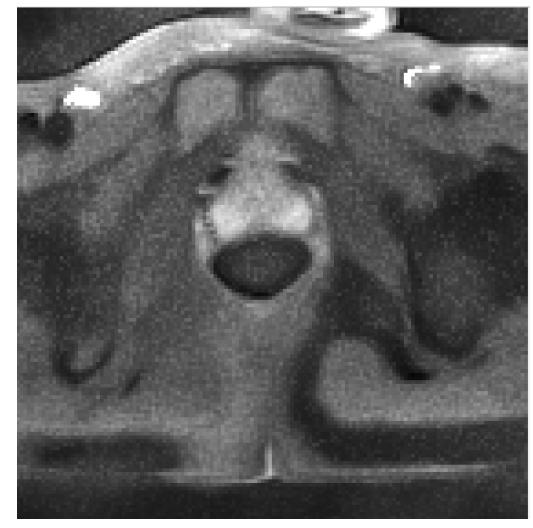

(b) Trace weighted image, b600, STEAM

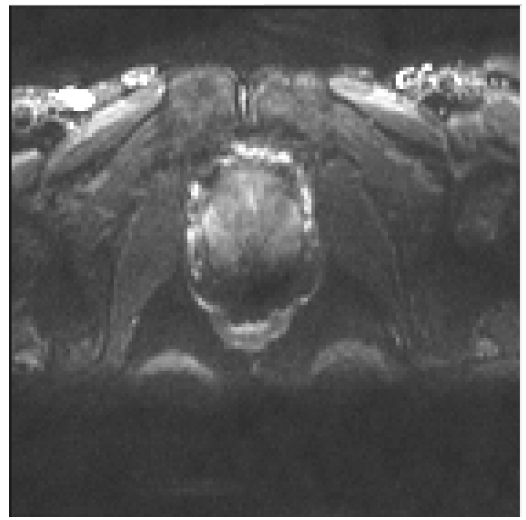

(e) Trace weighted image, b600, EPI

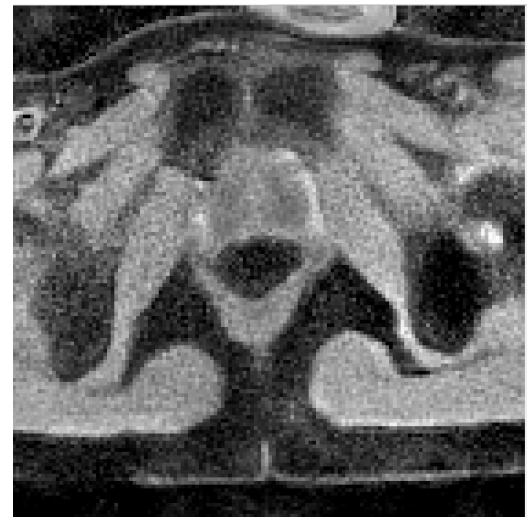

(c) ADC, STEAM

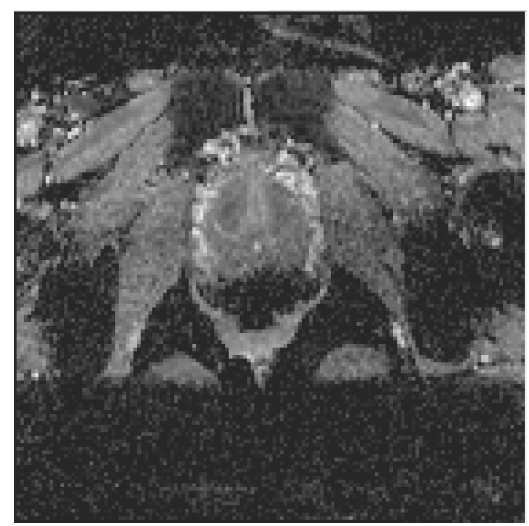

(f) ADC, EPI

Figure 50 - Comparison of STEAM acquisition with optimized parameters and a commercial EPI prostate protocol. Imaging parameters are shown in table 3 .

\subsection{Discussion}

The numerical simulations for validating the different variants of the reconstruction algorithm show that compared to magnitude averaging, direct application of the multi-shot reconstruction algorithm by Uecker et al. [61], i.e., multi-shot reconstruction without phase map smoothing, does 
not reduce the systematic error of ADC calculations from low-SNR data. This indicates that the inclusion of noisy phase maps in the multi-shot reconstruction model leads to a similar noise-related increase in diffusion-weighted image intensity as the averaging of magnitude images. Conversely, Uecker et al. report a substantial, visible increase in the SNR of diffusion-weighted images of the brain acquired with cartesian sampling which was achieved by their multi-shot reconstruction compared to single-shot reconstruction with averaging over magnitude images. The findings do not directly contradict each other, since Uecker et al. do not report quantitative values of the ADC or of a possible increase in diffusion-weighted image intensity. However, even if the image intensity is not affected, visibly lower SNR in the diffusion-weighted images should lead to higher statistical uncertainties of the ADC in single-shot reconstruction with magnitude averaging compared to multi-shot reconstruction without phase map processing. The simulations performed for this work do not show this. One possible reason is the application of the NLM denoising algorithm to the reconstructed images, which may even out the differences in the statistical errors.

The numerical simulations show that multi-shot reconstruction with an additional processing step to decrease the noise level of the phase maps solves the problem of accurate ADC calculation from data with low SNR. Both NLM denoising and k-spcae truncation reduce the minimal SNR for accurate ADC measurements by a factor of 2. The behavior of the ADC measured in the prostate as a function of slice thickness, i.e. an increase with increasing slice thickness which is removed by either of the two processing methods for phase maps, shows that the data generated in STEAM DWI of the prostate are in the critical SNR range where image reconstruction with phase map processing yields accurate ADC values, whereas image reconstruction without phase map processing leads to SNR-dependent systematic errors.

The remarkable robustness of the final reconstruction result to major qualitative differences in the reconstructed phase maps for NLM vs. k-space truncation shows that a further refinement of the phase map smoothing technique would probably have little practical effect on diffusion-weighted STEAM data of the prostate. NLM denoising has been chosen as the preferred method because it ensures that phase variations with high spatial frequencies are included in the reconstruction model. Such phase variations have been observed in the brain [61] and cannot be ruled out in the prostate. For data acquired with higher undersampling factors and higher SNR, k-space truncation or other methods for the removal of high spatial frequencies may be more suitable to reduce the undersampling artifacts in the phase maps.

Besides avoiding systematic errors in the measurement of the apparent diffusion coefficient, the multi-shot reconstruction technique with phase map processing allows higher undersampling factors and thereby enables the optimization of the choice of TR with respect to SNR efficiency, i.e. SNR per acquisition time. For the case of averaging, a number $m$ of image acquisitions would lead to an $S N R \sim \sqrt{m}$. For any sequence which depletes the longitudinal magnetization and is repeated multiple times, the SNR per acquisition is proportional to $1-e^{-T R / T 1}$. Hence, the SNR efficiency $\eta \sim \frac{1-e^{-T R / T 1}}{\sqrt{T R}}$, and $\eta$ is maximal for $T R=1.256 T 1$. For the prostate 
(central gland), $T 1=1.579 \pm 0.042 s$ at $3 \mathrm{~T}[$ [], i.e. the optimal TR would be $2 \mathrm{~s}$. However, this calculation neglects the depletion of longitudinal magnetization from the acquisition of neighboring slices. In particular, the low-angle pulses in the sequence for slice-selective excitation have a low bandwidth-time product and thus strongly deplete the longitudinal magnetization in the vicinity of the acquired slice. Moreover, the accuracy of the relation $S N R \sim \sqrt{m}$ is not guaranteed for the multi-shot reconstruction algorithm, where the SNR is balanced against spatial acuity with a regularization parameter. Hence, an experimental optimization on volunteers was necessary.

The results show that the image quality of a single diffusion-weighted image is robust to changes of TR between $4.2 \mathrm{~s}$ and $6.5 \mathrm{~s}$, while corruption by noise is substantially stronger for higher and lower repetition times. This allows the choice of TR from this range. A quantification of the SNR on these data was impractical, because measuring SNR with the difference method [9], i.e. estimating the noise level from the difference image from two subsequent acquisitions with identical imaging parameters, would lead to an unpredictable overestimation of the noise due to minor motion. Since the background noise is not homogeneous, the noise level can also not be reliably estimated from a background region. However, the robustness of the image quality with respect to changes in TR of up to $20 \%$ indicates that optimization strategies on quantitative SNR estimates would not substantially improve the quality of the results.

The experiment on the diffusion-weighted image quality and the ADC values as a function of the b-value clearly justifies the use of $b=600 \mathrm{~s} \mathrm{~mm} \mathrm{~mm}^{-2}$ instead of $b=400 \mathrm{~s} \mathrm{~mm}^{-2}$ and $b=800 \mathrm{~s} \mathrm{~mm}^{-2}$. The visible contrast in the resulting ADC and trace-weighted images is similar, while the SNR in the $b=800 \mathrm{~s} \mathrm{~mm}^{-2}$ image is much lower than in the $b=600 \mathrm{~s} \mathrm{~mm}^{-2}$ image. Even though the measured ADC in the prostate is known to decrease with increasing b-values [13], this cannot explain the observed global decrease of the ADC with increasing maximal b-value. This can be attributed to the low SNR in the $b=800 \mathrm{~s} \mathrm{~mm}^{-2}$ data leading to a systematic ADC underestimation. This result proves the advantage of setting the maximal b-value to $600 \mathrm{~s} \mathrm{~mm}^{-2}$ for prostate imaging. Moreover, ref. [13] shows that the sensitivity and specificity of prostate cancer diagnosis are comparable for $b=600 \mathrm{~s} \mathrm{mm^{-2 }}$ and. $b=1000 \mathrm{~s} \mathrm{~mm}^{-2}$.

The final STEAM imaging protocol (table 3) which was developed based on the experimental parameter optimization yields trace-weighted images and ADC maps which show a clearly defined and undistorted shape of the prostate, in particular near the intestinal wall. Despite the lower SNR compared to the EPI result, STEAM imaging has a significant potential for the correct diagnosis of tumors, particularly near the intestinal wall. Diagnostic errors due to susceptibility-induced image distortions and alterations of the image intensity are ruled out with use of the STEAM sequence. The practical diagnostic advantage from the avoidance of susceptibility artifacts has been shown in a study on stroke diagnosis [36]. This advantage is expected to be even more prominent in prostate imaging due to the higher gradients of magnetic susceptibility and the observed severe image distortions caused by the use of EPI. This work shows that undersampled radial STEAM DWI of the prostate is feasible. A clinical trial on patients with prostate cancer is clearly justified. 


\section{Diffusion-weighted STEAM MRI using inner-volume exci- tation}

\subsection{Introduction}

The prostate is located at the center of transverse sections through the lower abdomen and fills only a small part of the field of view of $200 \mathrm{~mm}$ used in the experiments described in section 5. The remaining tissue is a potential source of image artifacts which can also affect the image quality in the prostate. One example for this is streaking artifacts which can arise from tissue motion outside of the tissue of interest [5] leading to spoke-dependent dephasing and signal loss. Aditionally, alterations of the Larmor frequency due to magnetic susceptibilities, chemical shifts, or magnetic field inhomogeneities can cause streakings similar to those shown for the fat tissue in the scalp (see section 4.3.1).

Using inner-volume excitation, the field of view can be reduced to limit potential sources of such artifacts. An additional advantage is that imaging with a lower number of spokes is possible, because the undersampling factor with a fixed number of spokes is proportional to the width of the field of view. A previous method for STEAM imaging with inner-volume excitation [25] was extended to multiple slices to develop a protocol for inner-volume STEAM DWI of the prostate with the same coverage in the slice direction as the protocol for slice-selective excitation. The effect of inner-volume excitation on streaking artifacts and image quality was investigated in a study on four healthy volunteers and the physical sources of SNR loss in inner-volume excitation were characterized with theoretical calculations and experiments on phantoms.

\subsection{Methods}

\subsubsection{Imaging sequence}

The imaging sequence for inner-volume excitation as well as the difference with respect to the sequence for slice-selective excitation are shown in figure 51. For slice-selective excitation, all slice selection gradients are applied in the direction normal to the image section, and the sequence (preparation - readout) is repeated for multiple slices. For inner-volume excitation, slice selection gradients are applied in two perpendicular directions in the imaging plane for the diffusion-weighted spin echo and the second $90^{\circ}$ pulse. Hence, the preparation pulses excite a region of tissue which is bounded in the in-plane directions but not in the slice direction (see figure 52). Therefore, after the image acquisition for one slice, the longitudinal magnetization in all slices is depleted, and stimulated echoes with sufficient amplitudes can only be generated after waiting times on a scale of T1.

For this reason, multi-slice inner-volume STEAM DWI with acceptable acquisition times requires the readout of multiple slices after one application of the preparation module. To keep 
the same acquisition times as for slice-selective excitation, every second slice is read out following one preparation. This is done in an interleaved manner (see figure 51) to avoid differences in image contrast and SNR between the slices. A sequential readout of the slices would result in a slice-dependent time gap between preparation and readout, and a corresponding slice-dependent T1 relaxation, leading to a large variation with respect to both SNR and T1 contrast between the slices.

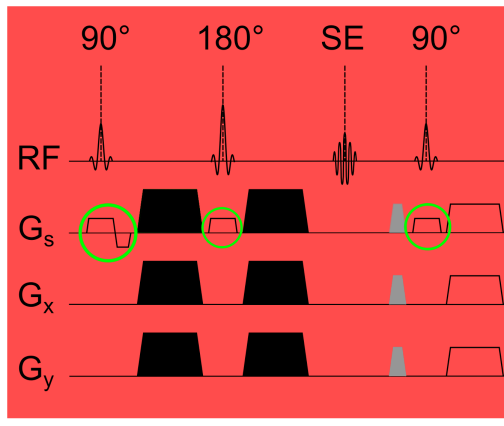

Preparation

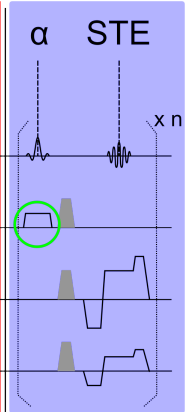

Readout

(a) The STEAM sequence with sliceselective excitation.

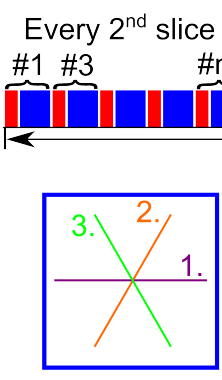

Slice \#1
Every $2^{\text {nd }}$ slice $\# \mathrm{~m} \quad \# 2 \quad \# 4 \quad \# \mathrm{~m}-1$ \#1 \#3 TR

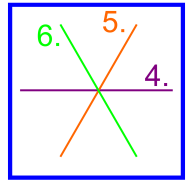

Slice \#3

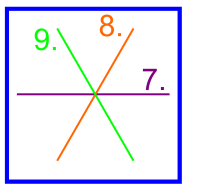

Slice \#5 (c) Sequential acquisition of multiple slices with slice-selective excitation.

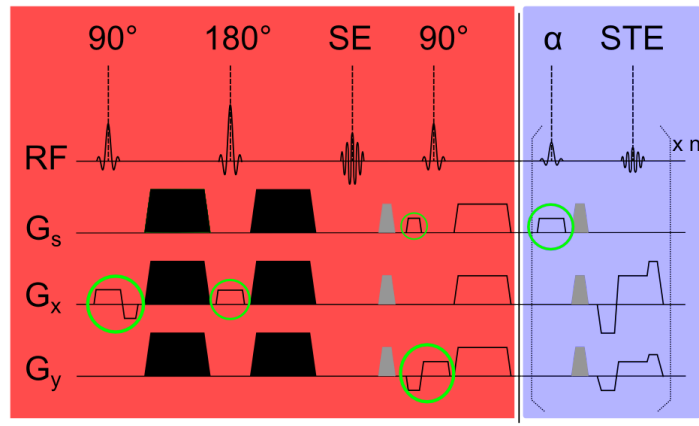

Preparation

Readout

(b) The STEAM sequence with innervolume excitation.

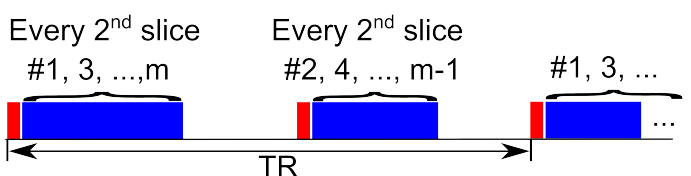

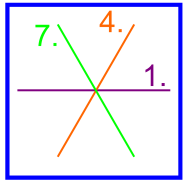

Slice \#1

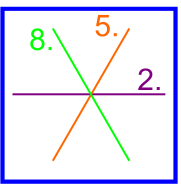

Slice \#3

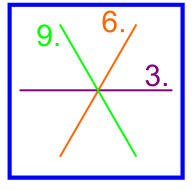

Slice \#5 (d) Interleaved acquisition with innervolume excitation.

Figure 51 - Image acquisition with slice-selective and inner-volume excitation. For sliceselective excitation, all RF pulses are selective in the slice direction. The preparation module (red) followed by the readout module (blue) is repeated for each slice. For inner-volume excitation, the diffusion-weighted spin echo is selective in one readout direction, the second $90^{\circ}$ pulse is selective in the perpendicular readout direction. The low-angle pulses for generating stimulated echoes are selective in the slice direction. Each preparation sequence block which prepares all slices is followed by a readout of multiple slices in an interleaved order, i.e. one radial spoke in k-space is read out for every second slice; this is repeated for all spokes. 


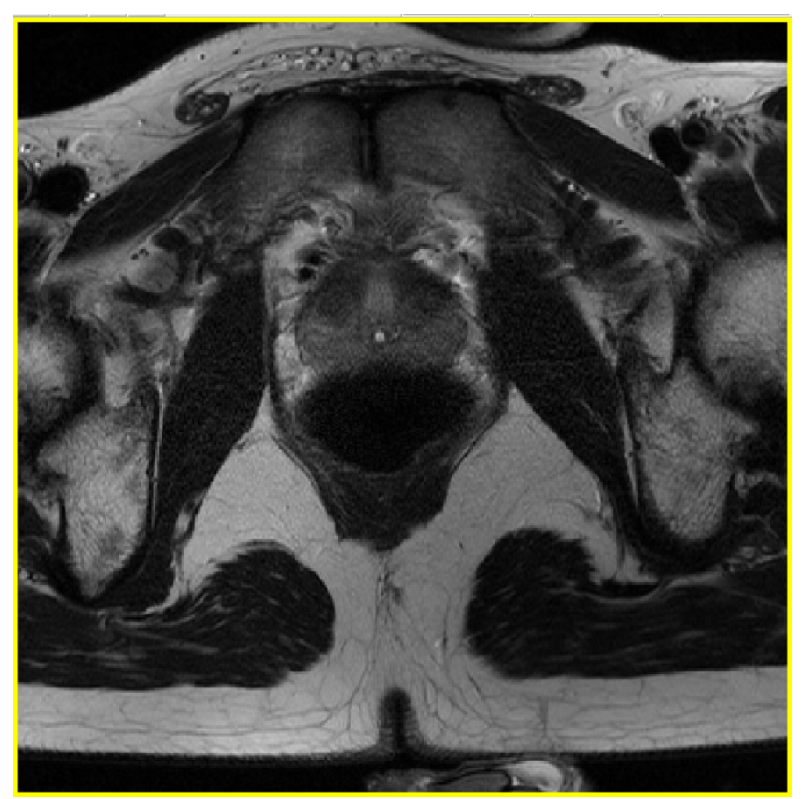

(a) Slice-selective excitation: same field of view as this anatomical image

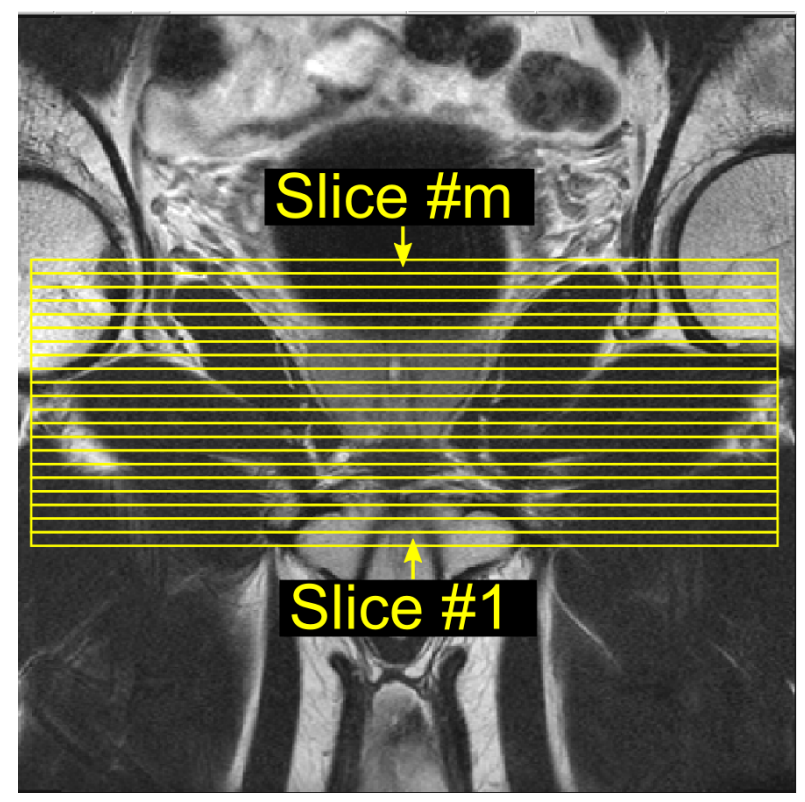

(c) The position of transverse slices shown on a coronal anatomical image for slice-selective excitation.

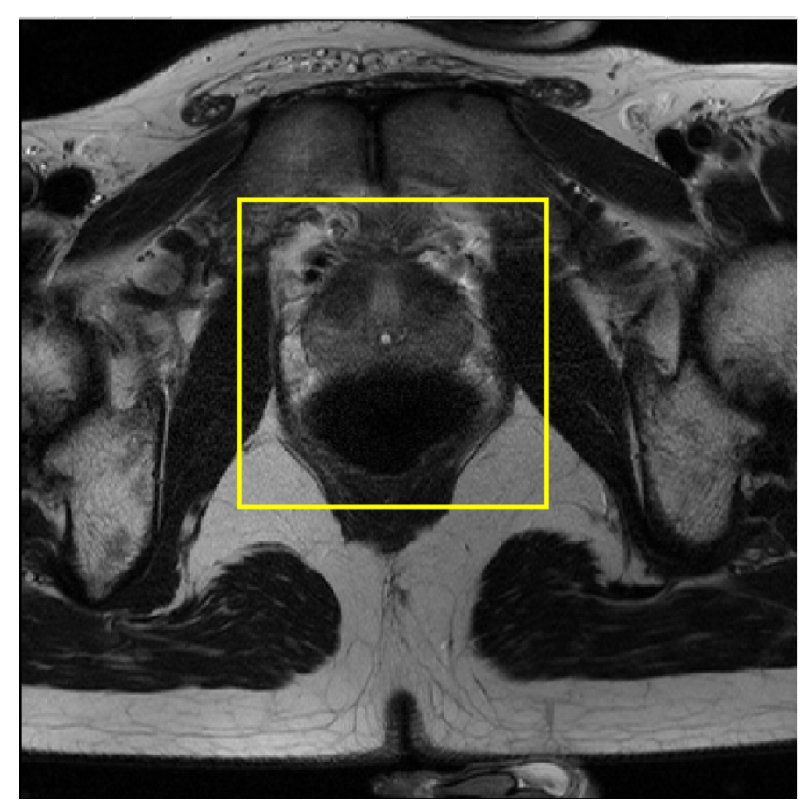

(b) Inner-volume excitation: the field of view is reduced to a region around the prostate

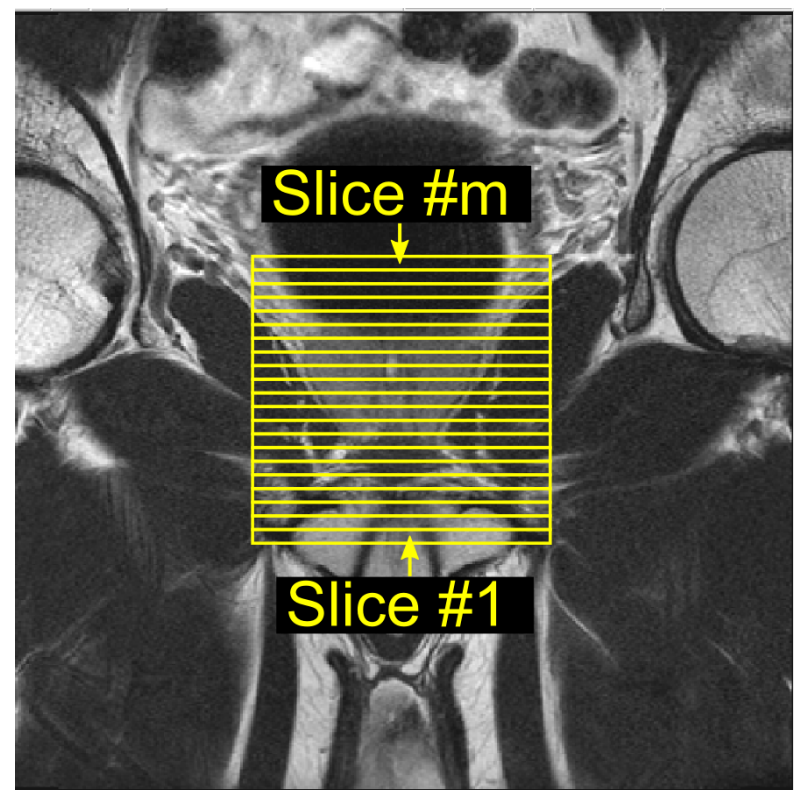

(d) The position of transverse slices shown on a coronal anatomical image for inner-volume excitation.

Figure 52 - Slice positions and fields of view for slice-selective excitation and inner-volume excitation. 


\subsubsection{Characterization of signal losses}

For a comparison of STEAM DWI of the prostate with inner-volume and slice-selective excitation, it is necessary to consider four effects which can lead to differences in SNR.

Firstly, the slice profile is defined only by the low-angle readout pulses if inner-volume excitation is used, whereas the slice profile with slice-selective excitation is the product of the slice profiles of the readout pulses and the preparation pulses.

Secondly, inner-volume selection leads to signal loss, particularly at the edges of the field of view, which is related to the slice profiles of the preparation pulses.

Thirdly, in the interleaved multi-slice readout module, each slice is read out with a repetition time $T R_{\alpha}$ multiplied by a factor $\mathrm{m}$, with $\mathrm{m}$ being the number of slices in the readout train. This causes major signal losses due to $\mathrm{T} 1$ relaxation. These signal losses can ameliorated by sampling a lower number of radial spokes for a reduced field of view.

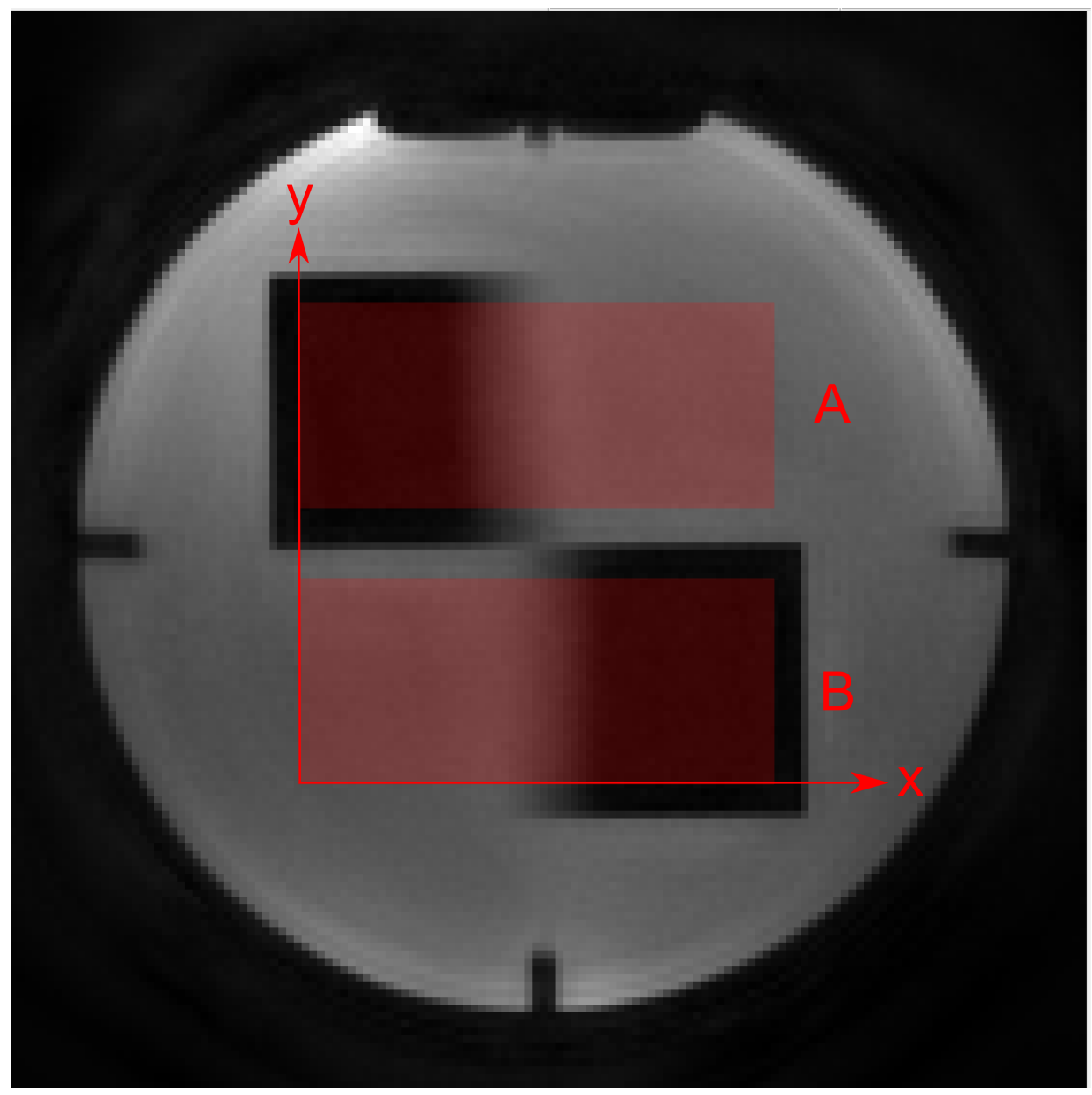

Figure 53 - Section of the phantom used for the measurement of slice profiles. The regions A and $\mathrm{B}$ are used for quantitative evaluation. 
Adaptation of the slice profile The slice profile of the inner-volume sequence was adapted to that of the slice-selective sequence. Slice profiles were measured using the Siemens structure phantom which contains a section filled with water and two plastic wedges oriented in opposite directions with a ramp of $11.3^{\circ}$. A MR image of a transverse slice of this region thus contains areas totally occupied by the wedges (dark, no MR signal), areas occupied by water (bright, much MR signal), and transition regions, where a fraction of the acquired slice is occupied by the wedge, see figure 53. The width of these transition regions is proportional to the slice thickness. Quantitatively, the slice profile, i.e. signal strength as a function of the $\mathrm{z}$ coordinate normal to the slice in a homogeneous sample, can be described in terms of the derivative of the intensity in the $\mathrm{x}$ direction in regions $\mathrm{A}$ and $\mathrm{B}$ (figure 53$) . S(z)=0.5\left(S_{A}(z)+S_{B}(z)\right.$ ) with $S_{A}(z)=$ $\tan (\alpha) \frac{d}{d x} M \circledast I_{A}(x)$ and $S_{B}(z)=-\tan (\alpha) \frac{d}{d x} M \circledast I_{B}(x) . \alpha=11.3^{\circ}$ is the angle of the wedges to the image plane, $M$ denotes a Gaussian kernel with which the measured slice profiles are convoluted to remove the influence of image noise, and $I_{A}=\langle I(x, y)\rangle_{y,(x, y) \in A}, I_{B}=\langle I(x, y)\rangle_{y,(x, y) \in B}$. The slice profiles were measured with slice-selective excitation using 35 radial spokes, a negligible b-value of $10 \mathrm{~s} \mathrm{mm^{-2 }}$ and 15 shots. To measure the expected slice profiles for inner-volume excitation, the same sequence was used, but the slice-selective gradients in the preparation module were switched off, so that only the readout pulses determine the slice profile. This measurement was repeated for bandwidth-time-products of the readout pulses between 2 and 5.5. The root-mean-square deviation to the slice profile from slice-selective excitation was measured for all inner-volume slice profiles, and the bandwidth-time product which yielded the lowest deviation was used for further experiments.

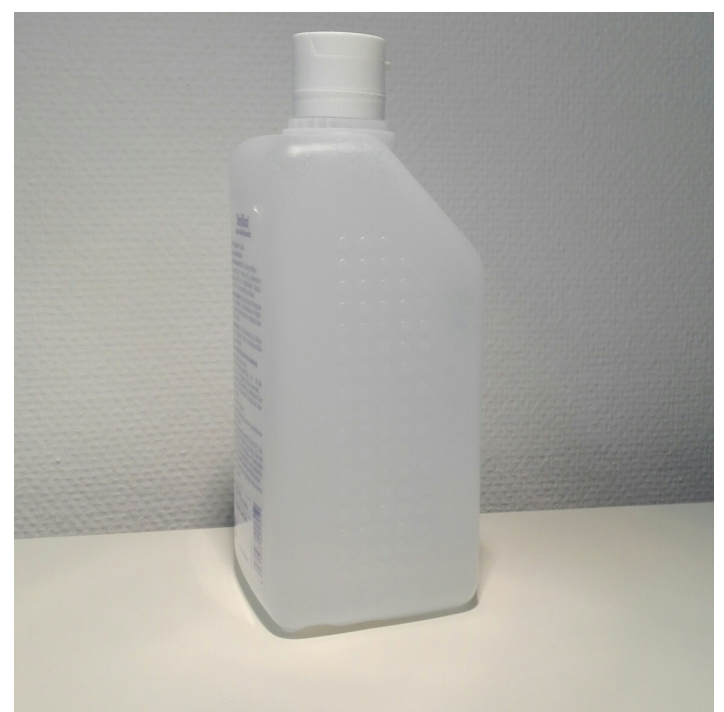

(a) The water-filled bottle used as a phantom

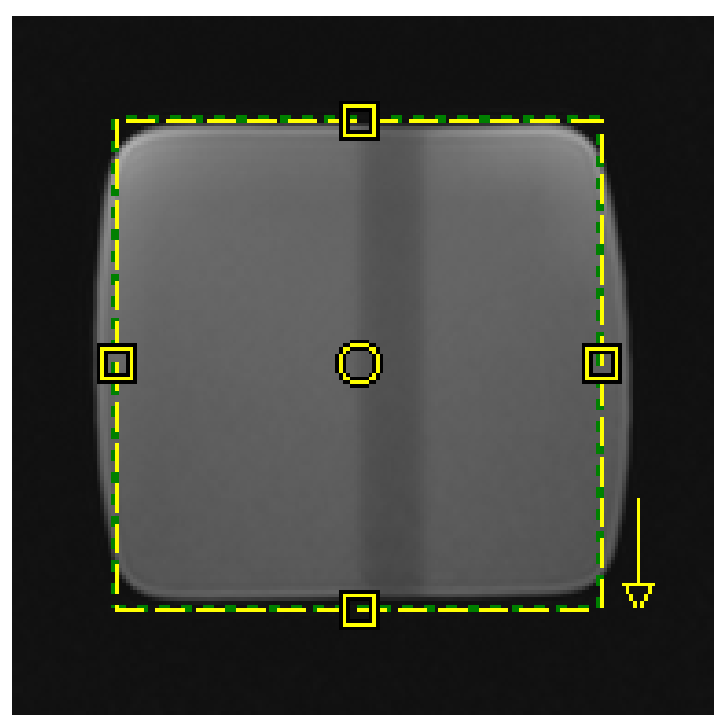

(b) The bottle matches the field of view of $80 \mathrm{~mm}$ used for inner-volume excitation

Figure 54 - The phantom to characterize the signal loss from inner-volume selection. 
Inner-volume selection The signal loss from inner-volume selection was measured using a plastic bottle filled with water which fits exactly into the dimensions of the desired square field of view with a width of $80 \mathrm{~mm}$ (figure 54). Two datasets with $\mathrm{b}=0$ and 19 radial spokes were acquired with inner-volume excitation $\left(A_{1}\right.$ and $\left.A_{2}\right)$, and two datasets were acquired with the same protocol, but with the gradients used for inner-volume selection switched off $\left(B_{1}\right.$ and $\left.B_{2}\right)$. The relative signal-to-noise ratios of the different acquisition modes was estimated as $S N R_{A}=\frac{\left\|A_{1}\right\|_{2}+\left\|A_{2}\right\|_{2}}{\sqrt{2}\left\|A_{1}-A_{2}\right\|_{2}}$, $S N R_{B}=\frac{\left\|B_{1}\right\|_{2}+\left\|B_{2}\right\|_{2}}{\sqrt{2}\left\|B_{1}-B_{2}\right\|_{2}}$, and the relative SNR loss $l=1-\frac{S N R_{A}}{S N R_{B}}$. To characterize the spatial dependence of the signal loss, images $I\left(A_{1}, A_{2}, B_{1}, B_{2}\right)$ were reconstructed from all four datasets using NLINV, and the magnitude ratio $R=\frac{\left|I\left(A_{1}\right)\right|+\left|I\left(A_{2}\right)\right|}{\left|I\left(B_{1}\right)\right|+\left|I\left(B_{2}\right)\right|}$ was computed.

Readout repetition times The effect of the prolonged repetition time $T R_{\alpha}$ and the number of spokes on the signal-to-noise ratio was estimated using theoretical calculations. In the variable flip angle model, the signal strength from all radial spokes $s=M_{1} \sin \left(\alpha_{1}\right)$ where $M_{1}$ is proportional to the initial longitudinally prepared magnetization, and $\alpha_{1}$ is given by the recursive formula $\alpha_{i}=\arctan \left(\sin \alpha_{i+1} e^{-\frac{T R_{\alpha}}{T 1}}\right)$, with $\alpha_{p}=\alpha_{\max }=40$ for $\mathrm{p}$ radial spokes. To estimate the expected effect of the signal strength $\mathrm{s}$ on the SNR of the raw data, the signal $S=s \sqrt{p}$ was computed, which is proportional to the expected $L_{2}$ norm of the raw data. The signal $\mathrm{S}$ was calculated with the parameters of the STEAM protocol for slice-selective excitation (see table 4), as well as for STEAM imaging with inner-volume excitation and a readout of 5 to 11 radial spokes, as a function of the number of slices in the readout train. Furthermore, a calculation was performed to estimate the slice thickness necessary to achieve inner-volume imaging of the prostate with an SNR equivalent to the SNR achieved with slice-selective excitation. As a function of the slice thickness $\mathrm{t}$, the number of slices $\mathrm{q}$ in the readout train necessary for a coverage of $z=21 * 3.5 \mathrm{~mm}$ in the slice direction (slice-selective excitation protocol) was first computed: $q=\left\lceil 0.5\left(\frac{z}{t}+1\right)\right\rceil$. The quantity $S$ was then computed from the parameters depicted in table 4, with 5 to 11 spokes, $T R_{\alpha}$ multiplied by $\mathrm{q}$, and the magnetization scaled proportionally to the slice thickness $\mathrm{t}$.

These signal strengths were compared to a target signal, which was defined as the simulated signal strength from the slice-selective excitation protocol, with a correction factor for signal loss due to inner-volume selection. To account for a signal loss $l$ as defined in the previous paragraph, the target signal must be increased by multiplication with $\frac{1}{1-l}$.

\subsubsection{In-vivo experiments}

For comparison of image quality of the diffusion-weighted images with and without inner-volume excitation, diffusion-weighted images of the prostate of a healthy volunteer were acquired with four different protocols, shown in table 4 :

1. Slice-selective excitation.

2. Inner-volume excitation, one slice. 
3. Inner-volume excitation, 21 slices.

4. Inner-volume-excitation with thicker slices expected to achieve an SNR equivalent to sliceselective excitation.

\begin{tabular}{|c|c|c|c|c|}
\hline Protocol No. & 1 & 2 & 3 & 4 \\
\hline Excitation mode & slice-selective & inner volume & inner volume & inner volume \\
\hline In-plane resolution [mm] & 1.43 & 1.43 & 1.43 & 1.43 \\
\hline Matrix size & 140 & 56 & 56 & 56 \\
\hline Field of view [mm] & 200 & 80 & 80 & 80 \\
\hline Slice thickness [mm] & 3.5 & 3.5 & 3.5 & 4.7 \\
\hline Number of slices & 21 & 21 & 21 & 17 \\
\hline b-value $\# 1\left[s \mathrm{~mm}^{-2}\right]$ & 50 & 50 & 50 & 50 \\
\hline Averages/shots \#1 & 7 & 7 & 7 & 7 \\
\hline b-value $\# 2\left[s \mathrm{~mm}^{-2}\right]$ & 600 & 600 & 600 & 600 \\
\hline Averages/shots \#2 & 17 & 17 & 17 & 17 \\
\hline Diffusion directions & 3 & 3 & 3 & 3 \\
\hline $\mathrm{TR}[\mathrm{ms}]$ & 5000 & 5000 & 5000 & 5000 \\
\hline Total acquisition time & $6 \min 25 \mathrm{~s}$ & $6 \min 25 \mathrm{~s}$ & $6 \min 25 \mathrm{~s}$ & $6 \min 25 \mathrm{~s}$ \\
\hline Bandwidth [Hz/Pixel] & 200 & 200 & 200 & 200 \\
\hline Radial spokes & 19 & 7 & 7 & 7 \\
\hline TSE [ms] & 30.8 & 29.9 & 29.9 & 29.9 \\
\hline $\mathrm{TR} \alpha[\mathrm{ms}]$ & 7.72 & 8.09 & 8.09 & 8.09 \\
\hline $\mathrm{TE}[\mathrm{ms}]$ & 8.92 & 8.96 & 8.96 & 8.96 \\
\hline
\end{tabular}

Table 4 - Optimized imaging protocols used for final comparisons of image quality.

In addition to this comparison of image quality, protocols 1 and 3 were run on four healthy

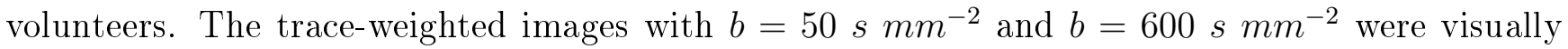
inspected for streaking artifacts and the numbers of corrupted image sections was counted to assess whether inner-volume excitation is useful and necessary for artifact-free prostate DWI. 


\subsection{Results}

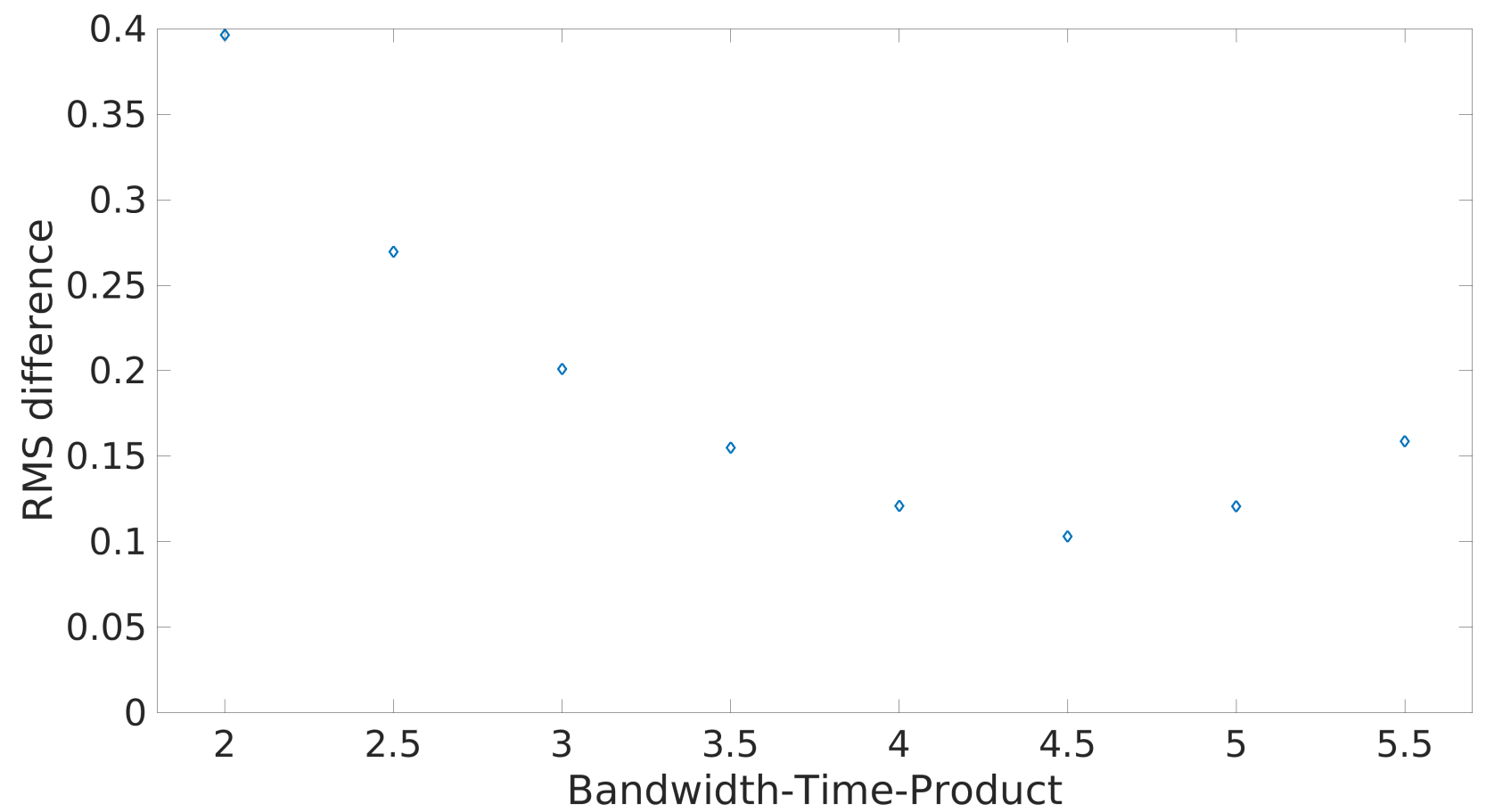

(a) RMS deviation between the slice profiles for inner-volume and slice-selective preparation as a function of the bandwidth-time product of the readout pulses after inner-volume preparation.

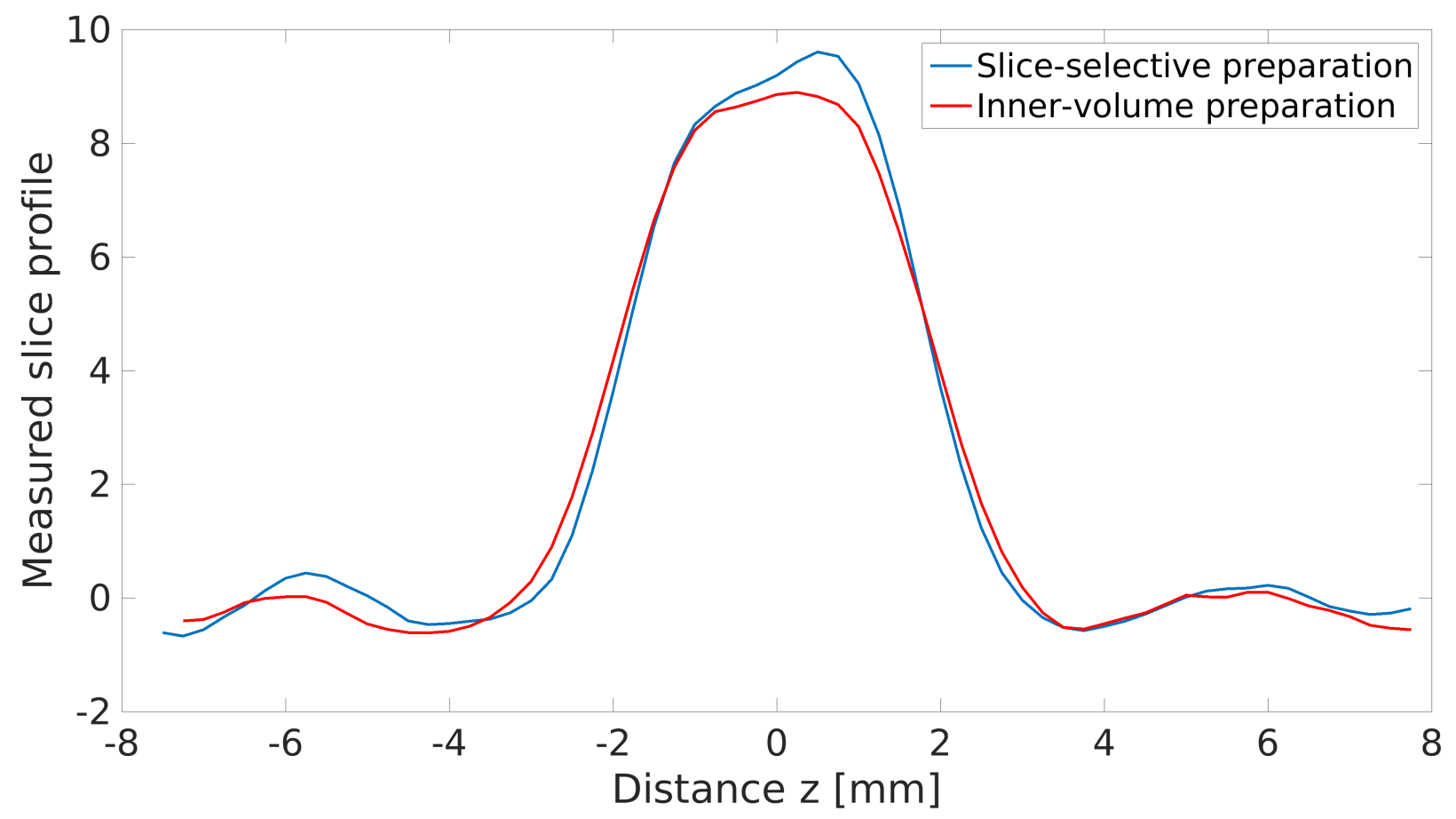

(b) Measured slice profiles for inner-volume preparation with a readout bandwidth-time-product of 4.5 and slice-selective preparation.

Figure 55 - Adaptation of slice profiles measured with inner-volume excitation to slice-selective excitation. 


\subsubsection{Characterization of signal losses}

Adaptation of the slice profile The measurement of the root-mean-square deviation between the slice profiles resulting from inner-volume vs. slice-selective excitation results in a minimal deviation for readout pulses with a bandwidth-time product of 4.5 after inner-volume excitation (figure 55a). The resulting slice profile, as well as the one measured for slice-selective excitation, are plotted in figure 55b. This plot shows that comparable slice profiles are achieved which do not cause substantial differences of image SNR or spatial resolution between the two sequence variants.

Inner-volume selection The single-slice experiment to characterize the loss in SNR due to imperfect slice profiles of the inner-volume-selective pulses resulted in a measured relative SNR loss in the raw data of $l=0.09$. The corresponding reduction of the L2-norm of the reconstructed images was also $9 \%$. Figure 56 shows the spatial distribution the intensity loss due to inner-volume selection. This shows a steep decrease in image intensity towards the boundaries of the field of view within a margin of $1 / 8$ of the image dimensions on each side, whereas the intensity in the image excluding this margin is well retained (L2-norm reduced by $3.1 \%$ ).

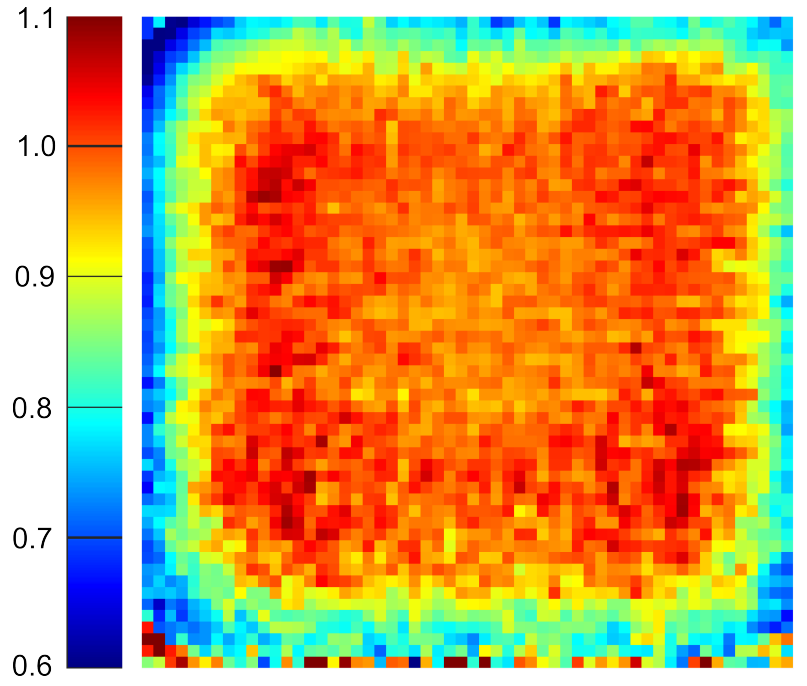

(a) Ratio of image intensities measured with and without inner-volume-selective gradients

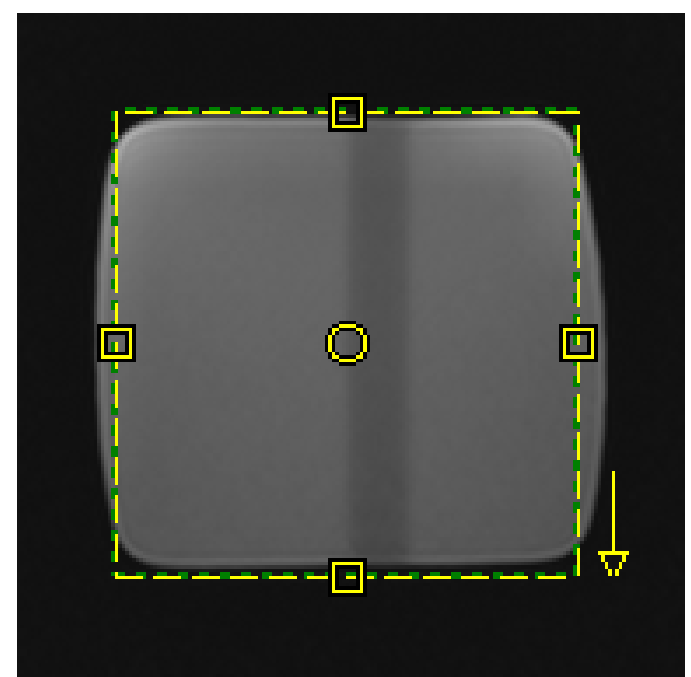

(b) Image section of phantom with field of view

Figure 56 - Characterization of signal loss due to inner volume selection.

Readout repetition times Calculations of estimated signal strength for inner-volume excitation show that for a readout train of 11 slices, which is necessary for a coverage of 21 slices, the signal strength is reduced with an increasing number of spokes due to T1 relaxation, whereas for one or two slices, the signal strength slightly increases with the number of spokes (figure $57 \mathrm{a}$ ). 


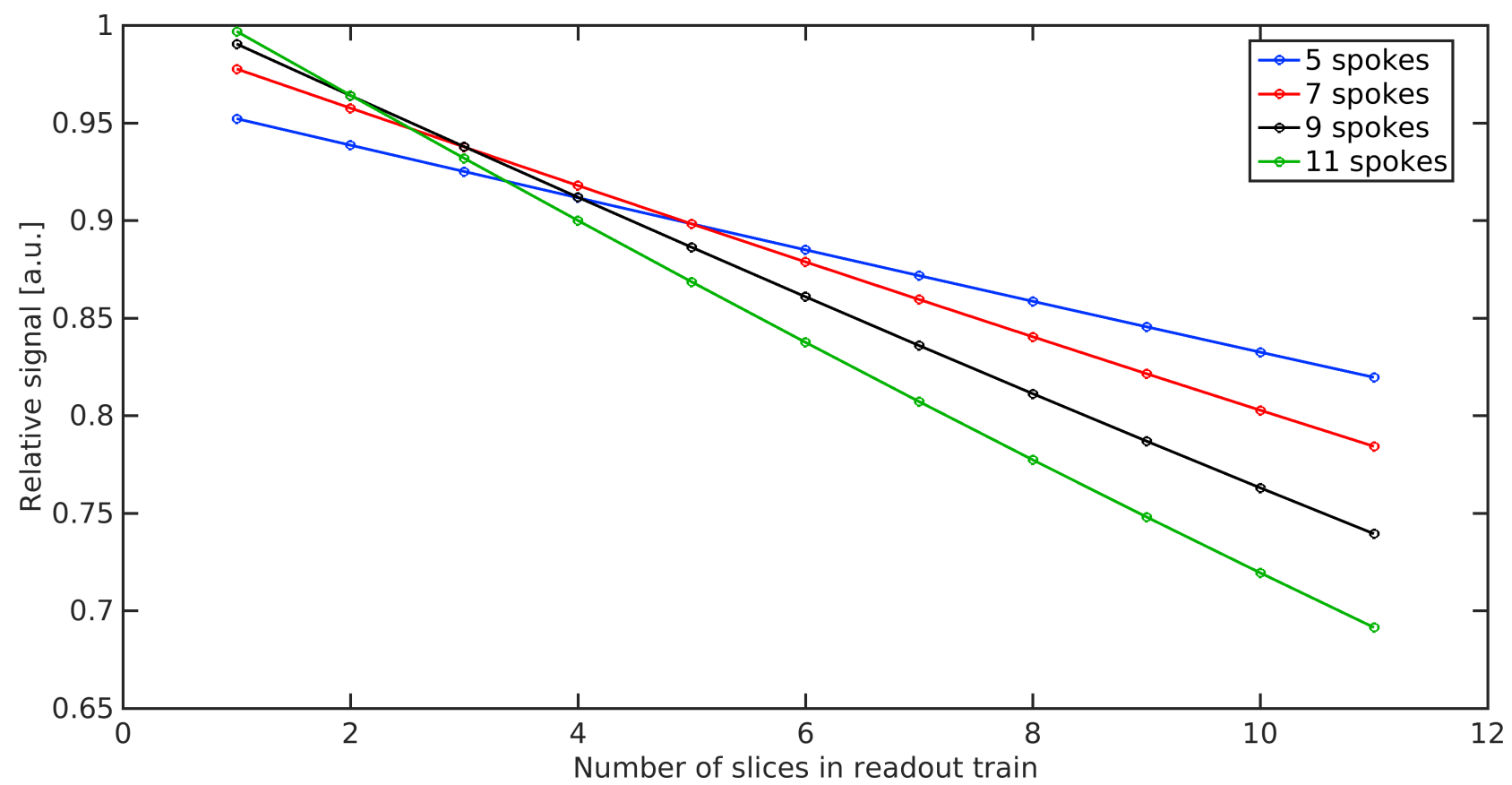

(a) Simulated signal strength for inner-volume excitation relative to slice-selective excitation with 19 spokes as a function of the number of slices in the readout train.

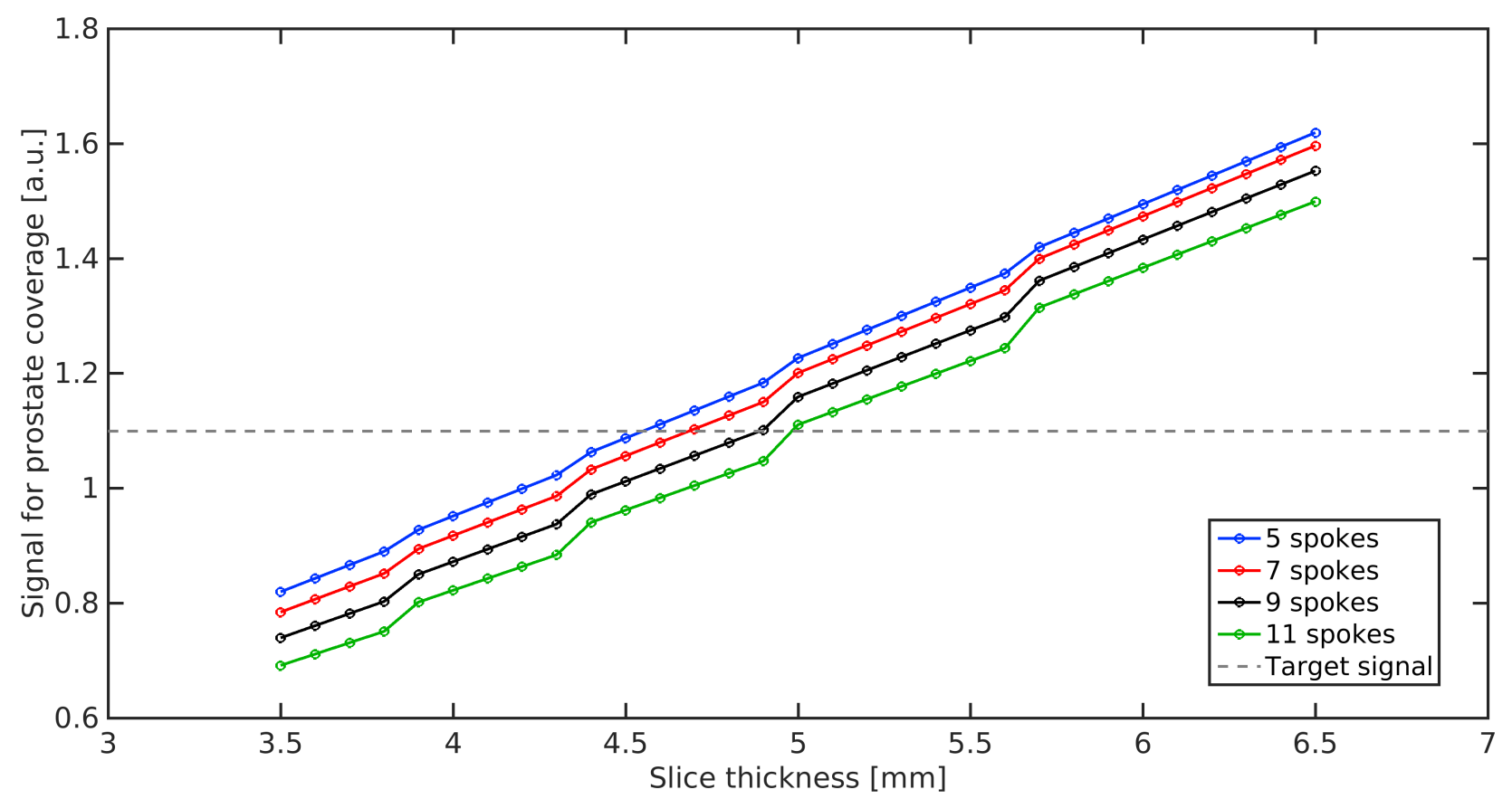

(b) Simulated signal strength for inner-volume excitation as a function of slice thickness; the numbers of slices in the readout train were chosen to allow for a volume coverage of $73.5 \mathrm{~mm}$. A signal strength of 1 is equivalent to the signal strength from one slice with a thickness of $3.5 \mathrm{~mm}$ acquired with 19 spokes. The target signal of 1.09 exceeds this signal strength to compensate for signal loss due to inner-volume selection.

Figure 57 - Simulated signal strength for inner-volume excitation as a function of slice thickness, with a coverage of $73.5 \mathrm{~mm}$ in the slice direction.

These calculations predict a reduction in signal strength of $21.6 \%$ for a readout train of 11 slices with 7 spokes as defined by protocol 3 in table 4 compared to single-slice readout with 19 
spokes (protocol 1). Simulations on the expected signal strength per slice for a coverage equivalent to $213.5 \mathrm{~mm}$ thick slices show that the slice thickness must be increased from 3.5 to $4.7 \mathrm{~mm}$ to compensate for the signal losses due to T1 decay and inner-volume selection (figure 57).

\subsubsection{In-vivo experiments}

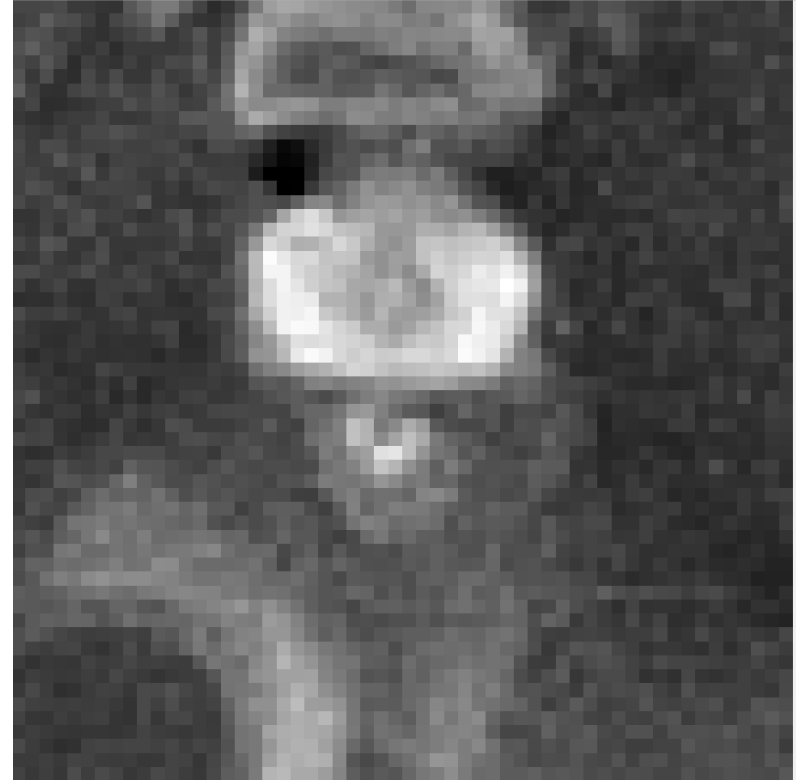

(a) Slice-selective excitation, slice thickness 3.5 mm, 19 spokes

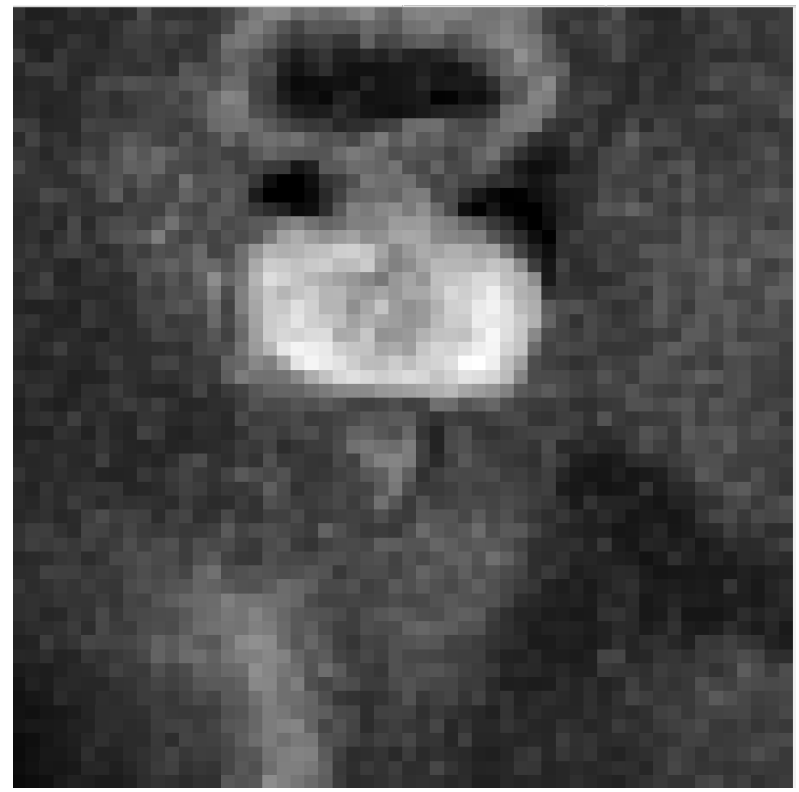

(c) Inner-volume excitation, 21 slices with a thickness of $3.5 \mathrm{~mm}, 7$ spokes

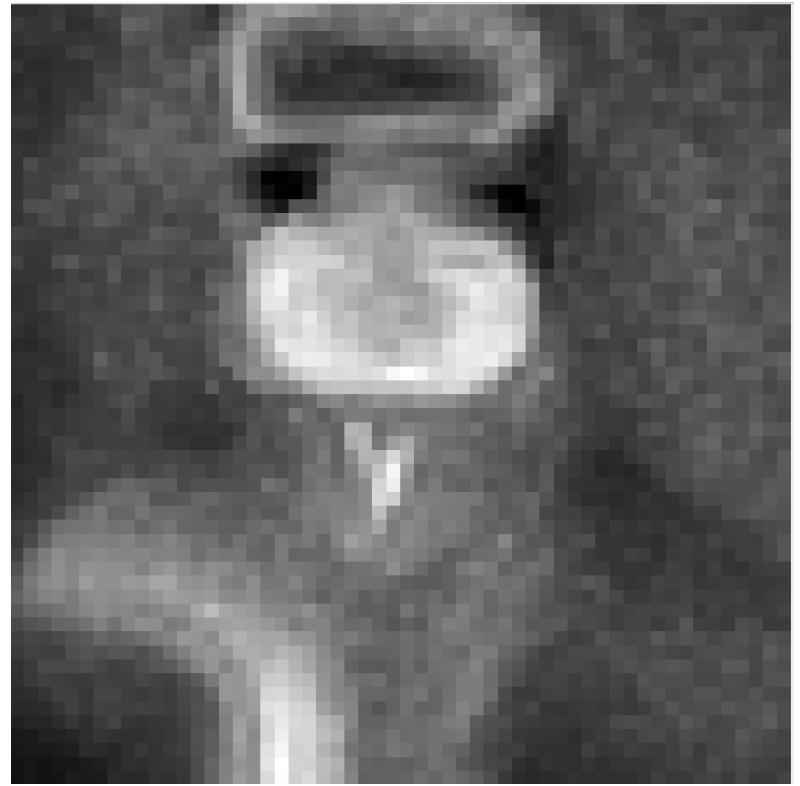

(b) Inner-volume excitation, single slice, 7 spokes

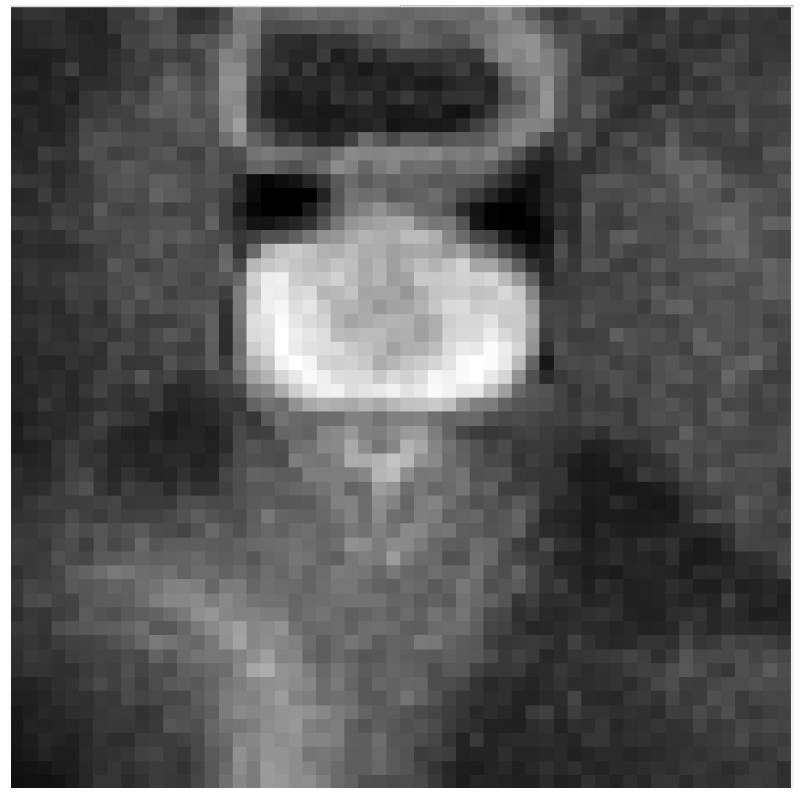

(d) Inner-volume excitation, 17 slices with a thickness $4.7 \mathrm{~mm}, 7$ spokes

Figure 58 - Trace weighted images of the prostate at $b=600 \mathrm{~s} \mathrm{~mm}^{-2}$ with slice-selective excitation and inner-volume excitation. 
Figure 58 shows the quality of trace-weighted images of the prostate acquired with slice-selective excitation with 19 spokes and inner-volume excitation with 7 spokes. Visual image inspection confirms that, compared to slice-selective excitation, inner-volume excitation with 1 slice does not lead to any substantial signal loss. In accordance with the simulation result, inner-volume imaging of 21 slices with a thickness of $3.5 \mathrm{~mm}$ leads to visibly reduced SNR. The image quality obtained using inner-volume excitation of 17 slices with a thickness of $4.7 \mathrm{~mm}$, i.e. the theoretically calculated slice thickness necessary to compensate for the SNR losses, is comparable to that resulting from the slice-selective excitation, as expected.

The experiment on the number of image sections corrupted by streaking artifacts for innervolume excitation vs. slice-selective excitation yielded the following results: None of the traceweighted images with $b=600 \mathrm{~s} \mathrm{~mm}^{-2}$ were affected by streakings. From a total of 47 traceweighted image sections acquired with $b=50 \mathrm{~s} \mathrm{~mm}^{-2}, 3$ sections were corrupted by streakings in inner-volume excitation mode. With the protocol using slice-selective excitation, the total number of corrupted sections was 4 . These included the 3 sections where inner-volume excitation also produced streakings, which were attributed to flow in the adjacent bladder. The trace-weighted image of the one section where streakings occurred only for slice-selective excitation is shown in figure 59. Figure 60 shows the trace-weighted images generated in a single-slice experiment where streakings from sources outside the prostate were suppressed due to inner-volume excitation. Here, the number of spokes was 15 for both excitation modes.

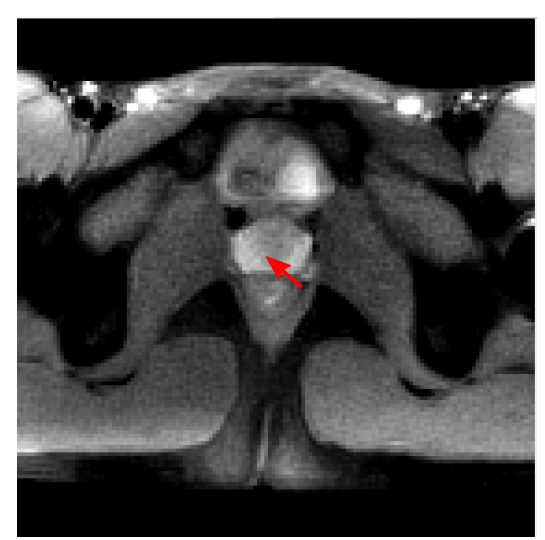

(a) Trace weighted image, b50, slice-selective excitation, full field of view

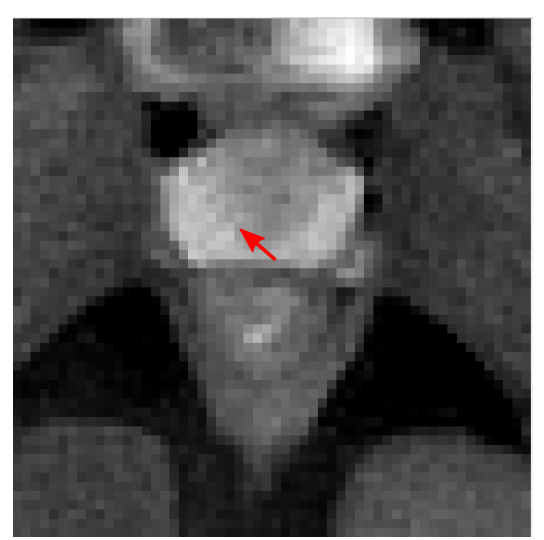

(b) Zoomed view of (a)

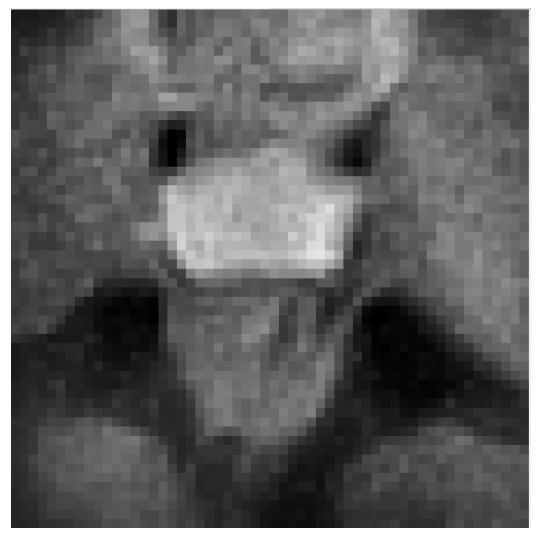

(c) Trace weighted image, b50, inner-volume excitation

Figure 59 - One slice from the multi-slice study on streaking reduction by inner-volume excitation. The streaking artifacts marked by the arrows are removed in the inner-volume excitation mode. 


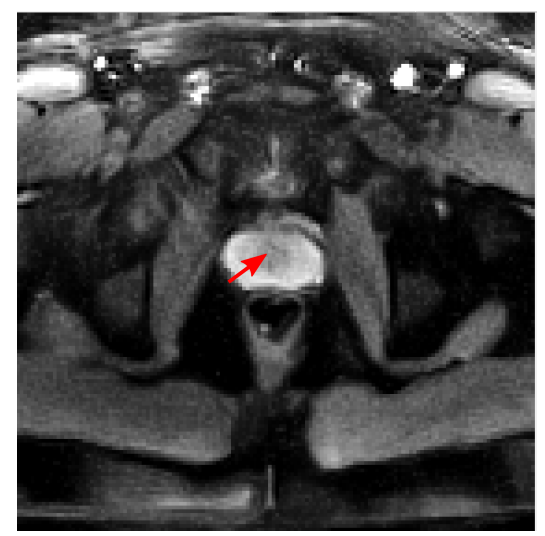

(a) Trace weighted image, b50, slice-selective excitation, full field of view.

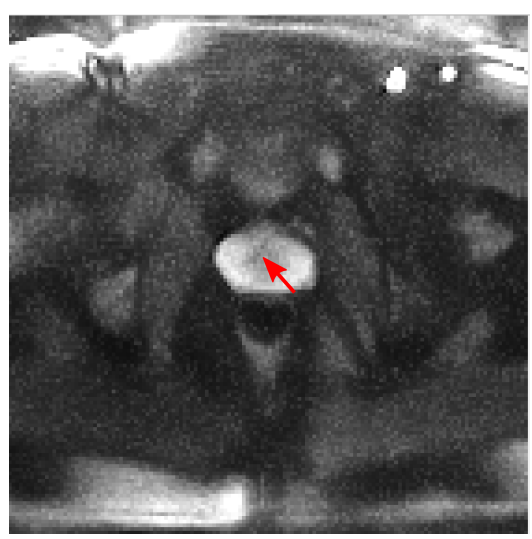

(d) Trace weighted image, b600, slice-selective excitation, full field of view.

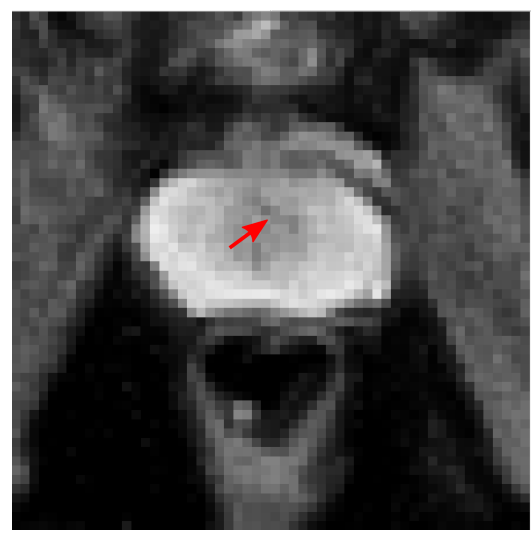

(b) View of (a) zoomed to 80 mm.

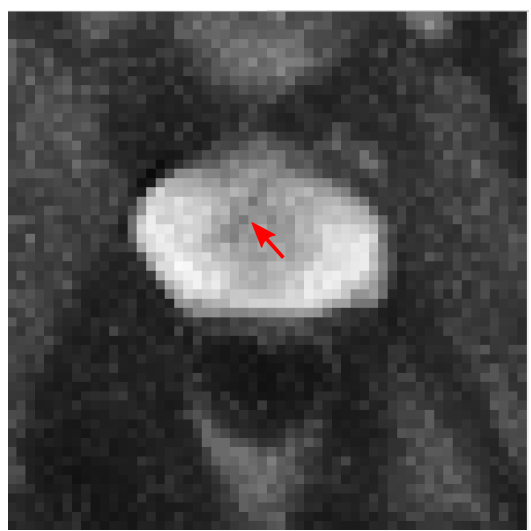

(e) View of (d) zoomed to 80 $\mathrm{mm}$.

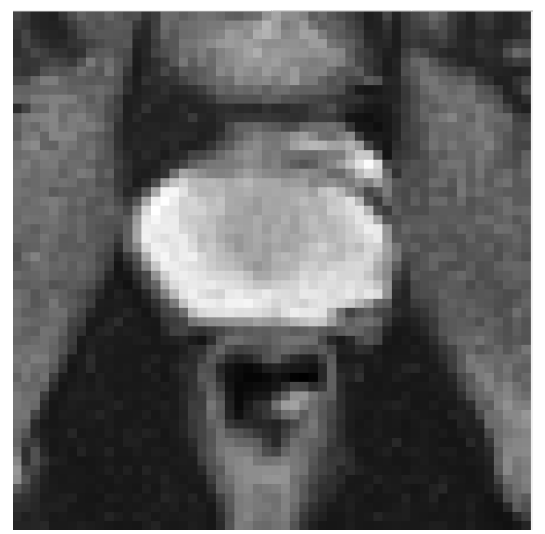

(c) Trace weighted image, b50, inner-volume excitation

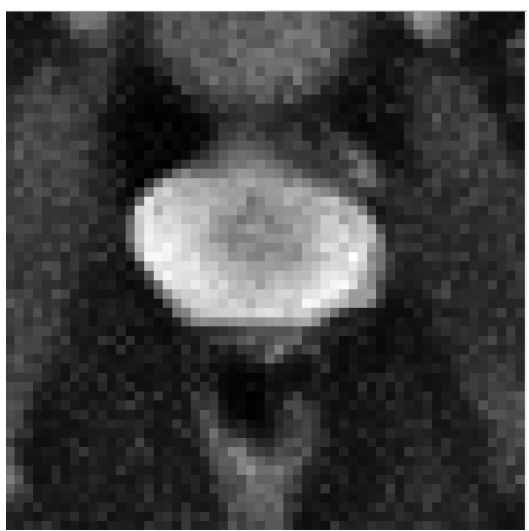

(f) Trace weighted image, b600, inner-volume excitation

Figure 60 - Reduction of streaking artifacts (red arrows) from outside the prostate due to inner-volume excitation. The data were acquired in a single-slice experiment with 15 radial spokes for both sequence variants. The field of view was $200 \mathrm{~mm}$ for slice-selective excitation and $80 \mathrm{~mm}$ for inner-volume excitation.

\subsection{Discussion}

For inner-volume excitation, the decay of image intensity towards the edges of the field of view indicates that the radiofrequency pulses in the preparation module of the sequence successfully discriminate between the field of view which is excited, and the surroundings, which are not excited. In-vivo images of the prostates of volunteers showed that this property can remove streaking artifacts which originate from tissue outside the prostate. However, a systematic comparison of the prostate images of four volunteers acquired with slice-selective excitation using optimized imaging protocols showed that streaking removal was necessary and effective in only one out of 47 image sections. Removal of streakings caused by flow in the bladder is difficult to achieve with this technique because the bladder is directly adjacent to the prostate. The novel multi-slice inner- 
volume imaging technique strongly reduces the image SNR compared to slice-selective excitation for a total coverage of 21 slices, despite the use of extremely undersampled radial trajectories to counteract signal losses from T1-relaxation. This clearly outweighs the advantage that a removal of occasional streakings may have for the quality of the resulting trace-weighted images and ADC maps. However, for one image slice, inner-volume excitation does not decrease the SNR and can therefore be judged as the favorable imaging method. Previously, single-slice inner-volume STEAM DWI with cartesian sampling has been successfully applied to the spine [25] and the rabbit shank [24]. For spine imaging, the technique developed for this work can offer the possibility of imaging multiple slices, and with a smaller number of slices (e.g. below 10), the SNR loss would not be as severe. In such a scenario, inner-volume imaging may be advantageous compared to slice-selective excitation because organs with respiratory and cardiac motion will be excluded from the tissue region which is excited. 


\section{$7 \quad$ Summary and outlook}

\subsection{Summary}

This thesis describes the successful development and in vivo application of a novel DW MRI technique that combines a single-shot STEAM MRI sequence with a strongly undersampled radial trajectory and an iterative image reconstruction by nonlinear inversion and optimized regularization. The results obtained for DW MRI of the human brain and prostate bear considerable potential to replace current clinical DW-MRI protocols based on EPI.

The improved single-shot STEAM MRI sequence on which these advances are based benefits from multiple methodological developments. For example, the newly introduced variable flip angle scheme and the modified spoiling method effectively suppress unwanted spurious signal contributions and related image artifacts. The combination of this single-shot STEAM sequence with radial undersampling and nonlinear inverse reconstruction regained SNR in comparison to a preceding proof-of-concept version based on Cartesian encoding and conventional image reconstruction. This non-DW sequence allowed for black-blood MRI of the human heart, despite a residual sensitivity of these measurements to myocardial motion.

Apart from further modifications of the STEAM sequence, the extension of the basic technique to DW single-shot STEAM MRI also required a refinement of the nonlinear inverse reconstruction. Together, the final strategy yielded excellent results for DW MRI of the human brain which particularly applies to the achievable image quality, spatial resolution, and measuring time for whole-brain studies. Because of the absence of susceptibility-induced image artifacts, DW singleshot STEAM MRI bears significant potential to replace DW-EPI sequences which currently are in exclusive clinical use.

In order to guarantee reproducible image quality under diverse brain MRI conditions, the iterative reconstruction process required innovations in the preprocessing of multi-slice datasets, in data scaling, and with respect to the initialization of estimated unknowns as well as an additional spatial regularization of coil sensitivities. In comparison with a recent brain study using DW singleshot STEAM MRI with conventional Cartesian encoding and image reconstruction by Fourier transformation, the new protocol offers improved image quality, while simultaneously allowing for either a reduction of the measuring time by a factor of 4 or a corresponding reduction of the voxel size, i.e. increased spatial resolution.

An extension of the DW single-shot STEAM MRI sequence to studies of the human prostate as a second clinically relevant application has to deal with low SNR as a special challenge. This is caused by the anatomical location of the prostate and the desire to use remote (rather than rectal) radiofrequency coils for signal reception to improve patient compliance and general acceptance.

The problem of accurate ADC quantification was solved by a multi-shot STEAM MRI technique using segmented radial k-space trajectories in conjunction with a joint reconstruction of multiple 
segments which involved the denoising of motion-associated phase maps to ensure phase consistency across segments. The resulting DW images and ADC maps of the prostate of healthy volunteers not only demonstrate the feasibility of DW multi-shot STEAM MRI, but further indicate a major benefit of the artifact-free STEAM technique in comparison to results obtained by a state-ofthe-art clinical DW-MRI protocol based on EPI. In particular, in contrast to EPI the absence of susceptibility-induced distortions ensures meaningful diagnostic information even along the critical boundary between the prostate and the air-filled colon.

Finally, preliminary applications of a newly developed DW multi-shot STEAM MRI sequence with spatially selective excitation for prostate studies within an inner volume showed promising results. Occasional artifacts from outer abdominal tissues with susceptibility differences, strong fat contributions or local tissue motion were effectively suppressed. So far, the approach suffers from reduced SNR for a large number of slices. The benefits of artifact suppression therefore outweigh the SNR penalty only for single-slice acquisitions, which are not suitable for clinical protocols covering the entire prostate.

\subsection{Outlook}

The results of this thesis demonstrate significant clinical potential of the developed DW STEAM MRI technique for diagnostic problems. The approach may replace applications hitherto served by EPI-based techniques which are prone to susceptibility-related artefacts. This potential should be further evaluated in clinical trials for DW MRI of brain and prostate. The inner-volume DW STEAM MRI method may be useful for spine MRI with multiple sections. Here, the exclusion of artifacts from surrounding tissue would effectively eliminate problems due to respiratory and cardiac motion. Clinical applications of the proposed DW STEAM MRI techniques will require an online implementation of the reconstruction algorithm using multiple graphical processing units as previously developed in our lab for real-time MRI.

Other future developments should explore a model-based mapping technique for the diffusion tensor which directly estimates all diffusion parameters from the raw data, for example by a nonlinear inverse reconstruction algorithm with an L1-based regularization of the motion-associated phase maps. This would avoid navigator echoes for phase map estimation which were necessary for a previously proposed model-based reconstruction of the diffusion tensor [39]. Another foreseeable extension of the DW STEAM MRI sequence is the use of many more gradient directions for diffusion encoding and the subsequent determination of pixel-wise orientation distribution functions (rather than simple diffusion tensors) with applications to nerve fiber tractography. This would enable new DW MRI studies and virtual fiber reconstructions in anatomical regions where EPI-based techniques are prone to susceptibility artifacts, for example, the optic nerve. 


\section{Abbreviations}

ADC apparent diffusion coefficient

BW bandwidth per pixel

BWTP bandwidth-time product

CHESS chemical shift selective

DW diffusion-weighted

DWI diffusion-weighted imaging

ECG electrocardiography

ENLIVE extended non-linear inversion inspired by ESPIRiT

EPI echo-planar imaging

FA fractional anisotropy

FID free induction decay

FLASH fast low angle shot

FOV field of view

GDC gradient delay correction

GPU graphics processing unit

FFT fast Fourier transform

GRAPPA generalized autocalibrating partial parallel acquisition

MRI magnetic resonance imaging

MR magnetic resonance

NLINV nonlinear inversion

NLM non-local means

NMR nuclear magnetic resonance

PCA principal components analysis

$\mathrm{RF}$ radiofrequency 
SNR signal-to-noise ratio

STEAM stimulated echo acquisition mode

T1 spin-lattice relaxation time

$\mathrm{T} 2 *$ effective spin-spin relaxation time

T2 spin-spin relaxation time

TE echo time

TM mixing time

$\mathrm{TR} \alpha$ readout repetition time

TR repetition time

TSE spin echo time 


\section{References}

[1] H E Assemlal, D Tschumperlé, L Brun, and K Siddiqi. Recent advances in diffusion MRI modeling: Angular and radial reconstruction. Medical Image Analysis, 15(4):369-396, 2011.

[2] F Balezeau, P A Eliat, A Bordelois Cayamo, and H Saint-Jalmes. Mapping of low flip angles in magnetic resonance. Physics in Medicine and Biology, 56(20):6635-6647, 2011.

[3] P J Basser, J. J Mattiello, and D LeBihan. Estimation of the effective self-diffusion tensor from the NMR spin echo. J Magn Reson B, 103(3):247-254, 1994.

[4] F Bloch. Nuclear induction. Physical Review, 70(7-8):460-474, 1946.

[5] K T Block. Advanced Methods fo Radial Data Sampling in Magnetic Resonance Imaging. PhD thesis, University of Göttingen, 2008.

[6] K T Block and J Frahm. Radial single-shot STEAM MRI. Magnetic Resonance in Medicine, 59(4):686-691, 2008.

[7] A Brander, A Kataja, A Saastamoinen, P Ryymin, H Huhtala, J Öhman, S Soimakallio, and $\mathrm{P}$ Dastidar. Diffusion tensor imaging of the brain in a healthy adult population: Normative values and measurement reproducibility at 3 t and 1.5 t. Acta Radiologica, 51(7):800-807, 2010 .

[8] C M J de Bazelaire, G D Duhamel, N M Neil M. Rofsky, and D C Alsop. MR imaging relaxation times of abdominal and pelvic tissues measured in vivo at 3.0 t: Preliminary results. Radiology, 230(3):652-659, 2004.

[9] O Dietrich, J G Raya, S B Reeder, M F Reiser, and S O Schoenberg. Measurement of signal-to-noise ratios in MR images: Influence of multichannel coils, parallel imaging, and reconstruction filters. Journal of Magnetic Resonance Imaging, 26(2):375-385, 2007.

[10] R R Edelman, D Chien, and D Kim. Fast selective black blood MR imaging. Radiology, 181(3):655-660, 1991.

[11] A Einstein. Über die von der molekularkinetischen theorie der wärme geforderte bewegung von in ruhenden flüssigkeiten suspendierten teilchen. Annalen der Physik, 322(8):549-560, 1905.

[12] S Emad-Eldin, M Halim, L I A Metwally, and R M Abdel-Aziz. Diffusion-weighted MR imaging and ADC measurement in normal prostate, benign prostatic hyperplasia and prostate carcinoma. The Egyptian Journal of Radiology and Nuclear Medicine, 45(2):535-542, 2014. 
[13] M Esen, M R Onur, N A, I Orhan, and E Kocakoc. Utility of adc measurement on diffusionweighted mri in differentiation of prostate cancer, normal prostate and prostatitis. Quantitative Imaging in Medicine and Surgery, 3(4):210-216, 2013.

[14] J Finsterbusch and J Frahm. Half-fourier single-shot STEAM MRI. Magnetic Resonance in Medicine, 47(3):611-615, 2002.

[15] J Frahm, W Hänicke, H Bruhn, M L Gyngell, and K D Merboldt. High-speed steam mri of the human heart. Magnetic Resonance in Medicine, 22(1):133-142, 1991.

[16] J Frahm, K D Merboldt, W Hänicke, and A Haase. Stimulated echo imaging. Journal of Magnetic Resonance (1969), 64(1):81-93, 1985.

[17] D T Ginat, M W Fong, D J Tuttle, S K Hobbs, and R C Vyas. Cardiac imaging: Part 1, MR pulse sequences, imaging planes, and basic anatomy. American Journal of Roentgenology, 197(4):808-815, 2011.

[18] M A Griswold, P M Jakob, R M Heidemann, M Nittka, V Jellus, J Wang, B Kiefer, and A Haase. Generalized autocalibrating partially parallel acquisitions (GRAPPA). Magnetic Resonance in Medicine, 47(6):1202-1210, 2002.

[19] E M Haacke, R W B, M R Thompson, and R Venkatesan. Magnetic Resonance Imaging: Physical Principles and Sequence Design. Wiley-Liss, 1999.

[20] A Haase, J Frahm, W Hanicke, and D Matthaei. $1 \mathrm{~h} \mathrm{nmr}$ chemical shift selective (chess) imaging. Physics in Medicine \& Biology, 30(4):341, 1985.

[21] A Haase, J Frahm, D Matthaei, W Hanicke, and K D Merboldt. FLASH imaging. rapid NMR imaging using low flip-angle pulses. Journal of Magnetic Resonance (1969), 67(2):258-266, 1986.

[22] E L Hahn. Spin echoes. Physical Review, 80(4):580-594, 1950.

[23] M R Hestenes and E Stiefel. Methods of conjugate gradients for solving linear systems. Journal of Research of the National Bureau of Standards, 49(6):409, 1952.

[24] P Hiepe, K H Herrmann, D Güllmar, C Ros, T Siebert, R Blickhan, K Hahn, and J R Reichenbach. Fast low-angle shot diffusion tensor imaging with stimulated echo encoding in the muscle of rabbit shank. NMR in Biomedicine, 27(2):146-157, 2013.

[25] P Hiepe, K H Herrmann, C Ros, and J R Reichenbach. Diffusion weighted inner volume imaging of lumbar disks based on turbo-STEAM acquisition. Zeitschrift für Medizinische Physik, 21(3):216-227, 2011. 
[26] S Hofer and J Frahm. Topography of the human corpus callosum revisited-comprehensive fiber tractography using diffusion tensor magnetic resonance imaging. NeuroImage, 32(3):989994, 2006.

[27] S Hofer, A Karaus, and J Frahm. Reconstruction and dissection of the entire human visual pathway using diffusion tensor MRI. Frontiers in Neuroanatomy, 4(15), 2010.

[28] H. Christian M. Holme, Sebastian Rosenzweig, Frank Ong, Robin N. Wilke, Michael Lustig, and Martin Uecker. Enlive: An efficient nonlinear method for calibrationless and robust parallel imaging. 20. Jahrestag. dtsch. Sekt. Int. Ges. Magn. Reson. Med. 2017, Göttingen, 2017.

[29] T A G M Huisman, T Loenneker, G Barta, M E Bellemann, J Hennig, J E Fischer, and K A Il'yasov. Quantitative diffusion tensor MR imaging of the brain: field strength related variance of apparent diffusion coefficient (ADC) and fractional anisotropy (FA) scalars. European Radiology, 16(8):1651-1658, 2006.

[30] S Hunsche, M E Moseley, P Stoeter, and M Hedehus. Diffusion-tensor MR imaging at 1.5 and 3.0 t: Initial observations. Radiology, 221(2):550-556, 2001.

[31] I O Jelescu and M D Budde. Design and validation of diffusion MRI models of white matter. Frontiers in Physics, 5, 2017.

[32] P Jezzard and R S Balaban. Correction for geometric distortion in echo planar images from b0 field variations. Magnetic Resonance in Medicine, 34(1):65-73, 1995.

[33] A K, S Hofer, and J Frahm. Separation of fiber tracts within the human cingulum bundle using single-shot STEAM DTI. The Open Medical Imaging Journal, 3(1):21-27, 2009.

[34] A Karaus, K D Merboldt, J Graessner, and J Frahm. Black-blood imaging of the human heart using rapid stimulated echo acquisition mode (steam) mri. Journal of Magnetic Resonance Imaging, 26(6):1666-1671, 2007.

[35] Alexander Karaus. Aufbau und Anwendung von Verfahren der Magnetresonanztomographie mit stimulierten Echos. PhD thesis, University of Göttingen, 2010.

[36] A A Khalil, M Hohenhaus, C Kunze, W Schmidt, P Brunecker, K Villringer, K D Merboldt, J Frahm, and J B Fiebach. Sensitivity of diffusion-weighted STEAM MRI and EPI-DWI to infratentorial ischemic stroke. PLOS ONE, 11(8):e0161416, 2016.

[37] P B Kingsley. Introduction to diffusion tensor imaging mathematics: Part III. tensor calculation, noise, simulations, and optimization. Concepts in Magnetic Resonance Part A, $28 \mathrm{~A}(2): 155-179,2006$. 
[38] J Klosowski and J Frahm. Image denoising for real-time MRI. Magnetic Resonance in Medicine, 77(3):1340-1352, 2016.

[39] F Knoll, J G Raya, R O Halloran, S Baete, E Sigmund, R Bammer, T Block, R Otazo, and D K Sodickson. A model-based reconstruction for undersampled radial spin-echo DTI with variational penalties on the diffusion tensor. NMR in Biomedicine, 28(3):353-366, 2015.

[40] P C Lauterbur. Image formation by induced local interactions: Examples employing nuclear magnetic resonance. Nature, 242(5394):190-191, 1973.

[41] D LeBihan and E Breton. Imagerie de diffusion in-vivo par résonance magnétique nucléaire. Comptes rendus de l'academie des sciences, 301(15):1109-1112, 1985.

[42] L Li, L Wang, M Deng, H Liu, J Cai, V K Sah, and J Liu. Feasibility study of 3-t DWI of the prostate: Readout-segmented versus single-shot echo-planar imaging. American Journal of Roentgenology, 205(1):70-76, 2015.

[43] P Mansfield. Multi-planar image formation using NMR spin echoes. Journal of Physics C: Solid State Physics, 10(3):L55-L58, 1977.

[44] K D Merboldt, W Hanicke, and J Frahm. Self-diffusion NMR imaging using stimulated echoes. Journal of Magnetic Resonance (1969), 64(3):479-486, 1985.

[45] A Merrem, S Hofer, D Voit, K D Merboldt, J Klosowski, M Untenberger, J Fleischhammer, and J Frahm. Rapid diffusion-weighted magnetic resonance imaging of the brain without susceptibility artifacts. Investigative Radiology, 52(7):428-433, 2017.

[46] O Natt. Entwicklung von Verfahren für in vivo NMR-Untersuchungen des Gehirns der Maus. PhD thesis, University of Göttingen, 2002.

[47] U G Nolte, J Finsterbusch, and J Frahm. Rapid isotropic diffusion mapping without susceptibility artifacts: Whole brain studies using diffusion-weighted single-shot steam mr imaging. Magnetic Resonance in Medicine, 44(5):731-736, 2000.

[48] D C Peters, J A Derbyshire, and E R McVeigh. Centering the projection reconstruction trajectory: Reducing gradient delay errors. Magnetic Resonance in Medicine, 50(1):1-6, 2003.

[49] J Pipe. Diffusion MRI, Second Edition: From Quantitative Measurement to In vivo Neuroanatomy. Academic Press, 2013.

[50] M Reiser and W Semmler, editors. Magnetresonanztomographie. Springer Berlin Heidelberg, 2002. 
[51] O Reynaud. Time-dependent diffusion MRI in cancer: Tissue modeling and applications. Frontiers in Physics, 5, 2017.

[52] E O Stejskal and J E Tanner. Spin diffusion measurements: Spin echoes in the presence of a time-dependent field gradient. The Journal of Chemical Physics, 42(1):288-292, 1965.

[53] J Talairach and P Tournoux. Co-Planar Stereotaxic Atlas of the Human Brain: 3-Dimensional Proportional System: An Approach to Cerebral Imaging. TPS, 1988.

[54] C H Tan, J Wang, and V Kundra. Diffusion weighted imaging in prostate cancer. European Radiology, 21(3):593-603, 2010.

[55] Z Tan. Advances in Real-Time Phase-Contrast Flow MRI and Multi-Echo Radial FLASH. PhD thesis, University of Göttingen, 2016.

[56] D G Taylor and M C Bushell. The spatial mapping of translational diffusion coefficients by the NMR imaging technique. Physics in Medicine and Biology, 30(4):345-349, 1985.

[57] H C Torrey. Bloch equations with diffusion terms. Physical Review, 104(3):563-565, 1956.

[58] B Turkbey, O Aras, N Karabulut, A T Turgut, E Akpinar, S Alibek, Y Pang, S M Erturk, R H El Khouli, D A Bluemke, and P L Choyke. Diffusion weighted MRI for detecting and monitoring cancer: a review of current applications in body imaging. Diagnostic and Interventional Radiology, 2011.

[59] M Uecker. Nonlinear reconstruction methods for parallel magnetic resonance imaging. PhD thesis, University of Göttingen, 2009.

[60] M Uecker, T Hohage, K T Block, and J Frahm. Image reconstruction by regularized nonlinear inversion-joint estimation of coil sensitivities and image content. Magnetic Resonance in Medicine, 60(3):674-682, 2008.

[61] M Uecker, A Karaus, and J Frahm. Inverse reconstruction method for segmented multishot diffusion-weighted MRI with multiple coils. Magnetic Resonance in Medicine, 62(5):1342$1348,2009$.

[62] M Uecker, S Zhang, and J Frahm. Nonlinear inverse reconstruction for real-time MRI of the human heart using undersampled radial FLASH. Magnetic Resonance in Medicine, 63(6):1456$1462,2010$.

[63] M Uecker, S Zhang, D Voit, A Karaus, K D Merboldt, and J Frahm. Real-time MRI at a resolution of $20 \mathrm{~ms}$. NMR in Biomedicine, 23(8):986-994, 2010. 
[64] M Untenberger. Multi-Echo Radial FLASH Techniques for Real-Time MRI. PhD thesis, University of Göttingen, 2015.

[65] J Wang, W Mao, M Qiu, M B Smith, and R T Constable. Factors influencing flip angle mapping in MRI: RF pulse shape, slice-select gradients, off-resonance excitation, andB0 inhomogeneities. Magnetic Resonance in Medicine, 56(2):463-468, 2006.

[66] X Wang, V Roeloffs, J Klosowski, Z Tan, D Voit, M Uecker, and J Frahm. Model-based t1 mapping with sparsity constraints using single-shot inversion-recovery radial FLASH. Magnetic Resonance in Medicine, 79(2):730-740, 2018.

[67] J P Wansapura, S K Holland, R S Dunn, and W S Ball. Nmr relaxation times in the human brain at 3.0 tesla. Journal of Magnetic Resonance Imaging, 9(4):531-538, 1999.

[68] K L Weiss, H Pan, J Storrs, W Strub, J L Weiss, L Jia, and O P Eldevik. Clinical brain MR imaging prescriptions in Talairach space: technologist- and computer-driven methods. AJNR Am J Neuroradiol, 24(5):922-929, May 2003.

[69] S Winkelmann, T Schaeffter, T Koehler, H Eggers, and O Doessel. An optimal radial profile order based on the golden ratio for time-resolved MRI. IEEE Transactions on Medical Imaging, 26(1):68-76, 2007.

[70] W Wu and K L Miller. Image formation in diffusion MRI: A review of recent technical developments. Journal of Magnetic Resonance Imaging, 46(3):646-662, 2017.

[71] J Xu, S W Sun, R T Naismith, A Z Snyder, A H Cross, and S K Song. Assessing optic nerve pathology with diffusion MRI: from mouse to human. NMR in Biomedicine, 21(9):928-940, 2008. 


\section{Curriculum Vitae}

\section{Personal Data}

- Name: Andreas Merrem

- Date of birth: 19.9.1986

\section{Studies}

- 2006: Abitur at Felix-Klein-Gymnasium Göttingen. Grade 1.3.

- 2007-10: Studies of physics (B.Sc.) at the University of Heidelberg. Final grade 1.4.

- 2009: Undergraduate research internship at the National Institute of Standards and Technology with an exchange program of the German Academic Exchange Service (DAAD)

- 2010-13: Studies of physics (M.Sc.) at the University of Heidelberg. Final grade 1.1.

- 2010-11: Academic exchange year at University College London

- Since January 2014: Doctoral studies at the Biomedizinische NMR Forschungs GmbH Göttingen within the doctoral program "IMPRS Physics of Biological and Complex Systems" of the Göttingen Graduate School of Neurosciences, Biophysics, and Molecular Biosciences (GGNB)

\section{Publications}

- A. Merrem, F. Zöllner, L. Schad: A variational approach to image registration in dynamic contrast-enhanced MRI of the human kidney. Magnetic Resonance Imaging, 2013, 31(5), $771-777$

- S. Li, F. G. Zöllner, A. Merrem, Y. Peng, J. Rørvik, A. Lundervold, L. Schad: Waveletbased segmentation of renal compartments in DCE-MRI of human kidney: Initial results in patients and healthy volunteers. Computerized Medical Imaging and Graphics, 2012, 36(2), $108-118$

- A. Merrem, S. Bartzsch, J. Laissue, U. Oelfke. Computational modelling of the cerebral cortical microvasculature: effect of x-ray microbeams versus broad beam irradiation. Physics in Medicine and Biology, 2017, 62(10): 3902-3922 
- A. Merrem, S. Hofer, D. Voit, K.-D. Merboldt, J. Klosowski, M. Untenberger, J. Fleischhammer, J. Frahm. Rapid diffusion-weighted magnetic resonance imaging of the brain without susceptibility artifacts: single-shot STEAM with radial undersampling and iterative reconstruction. Investigative Radiology, 2017, 52(7): 428-433

\section{Conference Contributions}

- Poster: A. Merrem, F. Zöllner and L. Schad. A variational approach to image registration in DCE-MRI of human kidney. Proc. Intl. Soc. Mag. Reson. Med., Montreal, Canada , 2011, 19:815

- Poster: F. Zöllner, S. Li, A. Merrem, J. Rørvik, A. Lundervold and L. Schad. Renal compartment segmentation by wavelet-based clustering of 3D DCE-MRI of human kidney. Proc. Intl. Soc. Mag. Reson. Med., Melbourne, Australia , 2012, 20:468

- Talk: A. Merrem, S. Bartzsch, J. Laissue and U. Oelfke. Can Computer Modelling of the Microvasculature help Understanding the MRT Tissue Sparing? MASR, Grenoble, France, 2015

- Talk: A. Merrem, J. Klosowski, S. Hofer, K.-D. Merboldt and J. Frahm: Diffusion-weighted MRI using undersampled radial STEAM with iterative image reconstruction. Proc. Intl. Soc. Mag. Reson. Med., Montreal, Singapore, 2016, 24:216

- Talk: A. Merrem, J. Klosowski, K.-D. Merboldt and J. Frahm: Diffusion-weighted MRI of the prostate without susceptibility artifacts: undersampled radial STEAM MRI, ESMRMB, Barcelona, Spain, 2017

\section{Thesis Papers}

- Bachelor's Thesis under the supervision of Prof. Dr. Lothar Schad, Medical Faculty Mannheim, University of Heidelberg. Title: "Registration of images for the measurement of kidney perfusion by dynamic contrast-enhanced magnetic resonance imaging"

- Master's Thesis under the supervision of Prof. Dr. Uwe Oelfke, German Cancer Research Center, University of Heidelberg. Title: "Modeling of damage to the vasculature of the cerebral cortex after microbeam radiation therapy" 


\section{Acknowledgements}

Zuerst möchte ich Prof. Dr. Jens Frahm für die Aufnahme in die Biomedizinische NMR Forschungs GmbH danken. So bekam ich die Möglichkeit, über ein wissenschaftlich interessantes und zugleich klinisch relevantes Thema der medizinischen Physik zu promovieren. Bei dieser Arbeit hat er mich kontinuierlich und intensiv unterstützt, mir aber auch wichtige Freiräume gelassen.

Ebenfalls danken möchte ich Prof. Dr. Marina Bennati und Dr. Florian Rehfeldt für die interessierte und freundliche Begleitung meiner Arbeit als Mitglieder meines Thesis Committee.

Mein besonderer Dank gilt Dr. Klaus-Dietmar Merboldt für wissenschaftliche Anregungen und Ideen, für die Weitergabe von wichtigem Erfahrungswissen über die STEAM-Bildgebung und nicht zuletzt für einige schöne und durchaus anstrengende Stunden auf dem Tennisplatz.

Zu großem Dank verpflichtet bin ich Dr. Sabine Hofer für ihre stete Hilfsbereitschaft, vor allem ihre biologischen Kenntnisse und ihre Erfahrung waren in meiner Arbeit bei der Beurteilung von MR-Bildern unverzichtbar.

Kurt Böhm danke ich für die Bereitstellung und Pflege der auch für diese Arbeit notwendigen IT-Infrastruktur.

Besten Dank an Sylke Wallbrecht für freundliche und kompetente Beratung und Unterstützung in Verwaltungsfragen.

Dr. Dirk Voit hat mir den Einstieg in die Programmierung von MRT-Pulssequenzen erleichtert und war mir stets ein hilfsbereiter Anprechpartner in allen "Sequenzfragen". Dafür vielen Dank.

Bei Dr. Oleksandr Kalentev, Sebastian Schätz und Christian Holme möchte ich mich für Tipps zu Git und $\mathrm{C}++$ bedanken.

Allen Kolleginnen und Kollegen der Biomedizinischen NMR ForschungsGmbH danke ich für

gute Zusammenarbeit, viele fachliche Diskussionen, eine freundliche Arbeitsatmosphäre und eine schöne gemeinsame Zeit.

Auch meinen Probanden, ohne die diese Arbeit nicht möglich gewesen wäre, möchte ich an dieser Stelle ausdrücklich danken.

Mein gößter Dank gilt meiner Familie und meine Freundin Eva, die mir immer zur Seite standen. 